University of Tennessee Health Science Center UTHSC Digital Commons

\title{
Antibiotic Drug Discovery with an Eye Towards Overcoming Drug Resistance
}

Daniel Towner Hoagland

University of Tennessee Health Science Center

Follow this and additional works at: https://dc.uthsc.edu/dissertations

Part of the Bacteria Commons, Medicinal and Pharmaceutical Chemistry Commons, and the Pharmaceutical Preparations Commons

\section{Recommended Citation}

Hoagland, Daniel Towner (http://orcid.org/0000-0001-7260-4522), "Antibiotic Drug Discovery with an Eye Towards Overcoming Drug Resistance" (2016). Theses and Dissertations (ETD). Paper 409. http://dx.doi.org/10.21007/etd.cghs.2016.0459. 


\title{
Antibiotic Drug Discovery with an Eye Towards Overcoming Drug Resistance
}

\author{
Abstract \\ As a species, humans have become ever reliant on the use of antibiotics to facilitate our everyday lives. \\ The widespread emergence of resistance to currently used antibiotics is commonly attributed to an over \\ use in our society. Such resistance, coupled with a lack of innovation and production of novel antibiotic \\ drugs, threatens to return humanity to an era similar to one before the discovery of the first antibiotics. \\ The need to find new agents to be used in this fight is paramount, as well as learning from our recent \\ failures to produce such compounds. This document will highlight my efforts to contribute to the field of \\ antibiotic drug discovery from a medicinal chemist's perspective. Chapter one will be a brief survey of the \\ current state of antibiotics. There will be a brief description of various classes of drugs used and some of \\ their pharmacology. The second chapter will focus on the specific field of drug discovery for the pathogen \\ Mycobacterium tuberculosis, and some of the difficulties associated with targeting this particular \\ organism. This includes two classes of inhibitors that have distinct mechanisms of action from \\ commonly used anti-tubercular compounds. These series have \\ distinctly different paths to clinical relevance; one seeks improved drug-like properties and the other \\ seeks unique potency at an old target. The third chapter highlights a structure guided design of new anti- \\ folate compounds, a reinvestigation of known inhibitors of this biosynthetic pathway and exploratory \\ repurposing of compounds active against malaria. This chapter also holds a section on chemical \\ repurposing, a technique becoming increasingly useful in the field of antibiotic discovery. The fourth and \\ final chapter will be a synopsis and recollection of my graduate work and on the field of antibiotic drug \\ discovery in general.

\section{Document Type} \\ Dissertation \\ Degree Name \\ Doctor of Philosophy (PhD)

\section{Program} \\ Pharmaceutical Sciences \\ Research Advisor \\ Richard Lee, Ph.D.

\section{Keywords} \\ Antibiotics, Drug Discovery, Medicinal Chemistry

\section{Subject Categories} \\ Bacteria | Chemicals and Drugs | Medicinal and Pharmaceutical Chemistry | Medicine and Health \\ Sciences | Organisms | Pharmaceutical Preparations | Pharmacy and Pharmaceutical Sciences
}




\title{
Antibiotic Drug Discovery with an Eye towards Overcoming Drug Resistance
}

\author{
A Dissertation \\ Presented for \\ The Graduate Studies Council \\ The University of Tennessee \\ Health Science Center \\ In Partial Fulfillment \\ Of the Requirements for the Degree \\ Doctor of Philosophy \\ From The University of Tennessee
}

By

Daniel Towner Hoagland

August 2016 
Copyright (C) 2016 by Daniel Towner Hoagland. All rights reserved. 


\section{DEDICATION}

I would like to dedicate this work to my grandfather, Richard Hoagland. His last few years he couldn't tell me or anyone close enough to listen, how proud he was that I was working at St Jude and becoming a doctor. I know that he would love to have the seen the day I finish, so I dedicate this writing to him and the rest of my family. 


\section{ACKNOWLEDGEMENTS}

I would like to take some time to acknowledge a few people who have been incredibly supportive in my journey towards finishing my doctorate. Without these individuals support and guidance I would have undoubtedly fallen short of my goals, and would not be shaped to be the person or scientist I am today. First and foremost I would like to thank my research advisor, Dr. Richard Lee. Richard has been a profound influence both academically and personally, teaching me how to think about and conduct scientific research while at the same time staying grounded in the important things in life. Richard is a passionate gardener and rugby fan while maintaining an incredibly efficient and productive lab, all while exuding a calm demeanor and positive culture. This type of balance is something I strive to achieve going forwards and it has been incredible to witness it first hand for the past five years. Next I would like to credit the love and affection of my girlfriend, Robyn Umans. Robyn is genuinely the most supportive person I have ever met, and I am so fortunate to have found her and have her with me throughout this process. Without her calming influence these past few months would have been infinitely more stressful and difficult, and I think our perseverance through the long distance relationship we've had since I have been finishing has only made both of us stronger. Lastly the encouragement of my friends and family, especially my mother Mary, has been a vital aspect of me pursuing my ambition of becoming a PhD. I owe any and all my accomplishments to the support of the people that matter most to me, and am forever grateful to them. 


\begin{abstract}
As a species, humans have become ever reliant on the use of antibiotics to facilitate our everyday lives. The widespread emergence of resistance to currently used antibiotics is commonly attributed to an over use in our society. Such resistance, coupled with a lack of innovation and production of novel antibiotic drugs, threatens to return humanity to an era similar to one before the discovery of the first antibiotics. The need to find new agents to be used in this fight is paramount, as well as learning from our recent failures to produce such compounds. This document will highlight my efforts to contribute to the field of antibiotic drug discovery from a medicinal chemist's perspective. Chapter one will be a brief survey of the current state of antibiotics. There will be a brief description of various classes of drugs used and some of their pharmacology. The second chapter will focus on the specific field of drug discovery for the pathogen Mycobacterium tuberculosis, and some of the difficulties associated with targeting this particular organism. This includes two classes of inhibitors that have distinct mechanisms of action from commonly used anti-tubercular compounds. These series have distinctly different paths to clinical relevance; one seeks improved drug-like properties and the other seeks unique potency at an old target. The third chapter highlights a structure guided design of new anti-folate compounds, a reinvestigation of known inhibitors of this biosynthetic pathway and exploratory repurposing of compounds active against malaria. This chapter also holds a section on chemical repurposing, a technique becoming increasingly useful in the field of antibiotic discovery. The fourth and final chapter will be a synopsis and recollection of my graduate work and on the field of antibiotic drug discovery in general.
\end{abstract}




\section{TABLE OF CONTENTS}

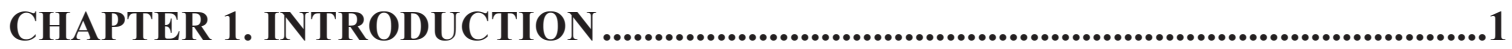

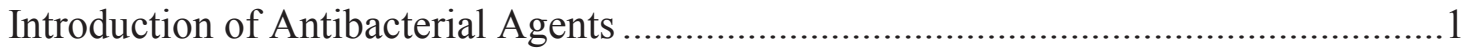

Inhibitors of the Folate Biosynthesis Pathway.........................................................

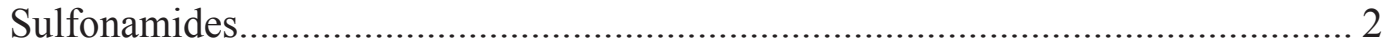

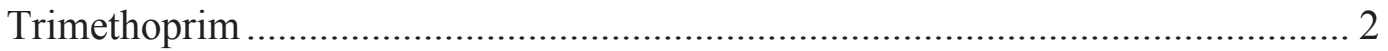

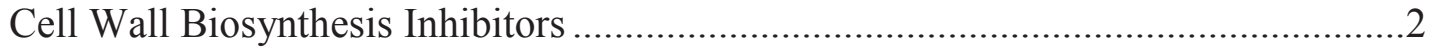

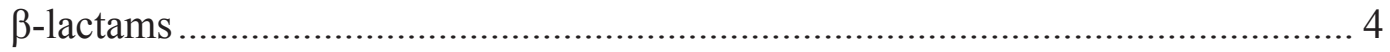

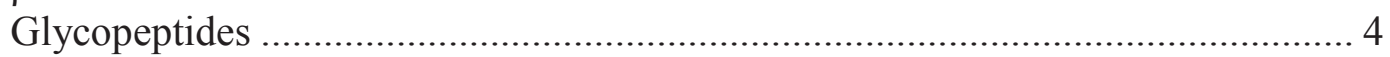

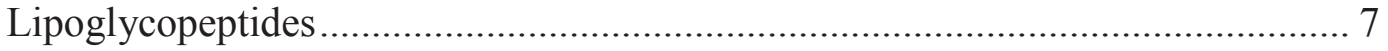

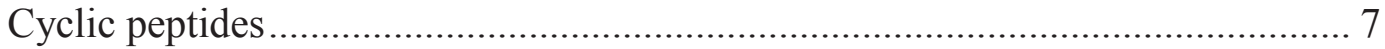

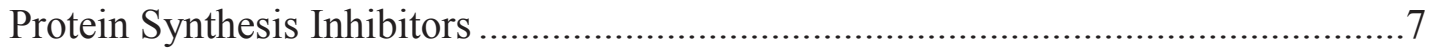

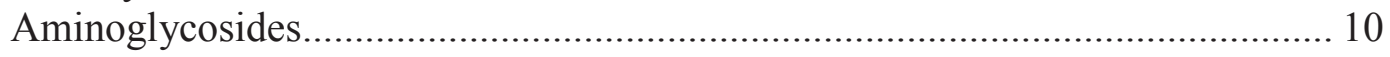

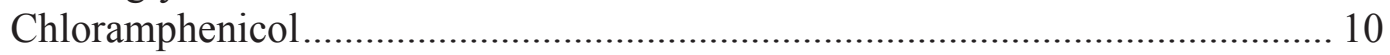

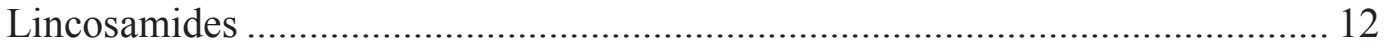

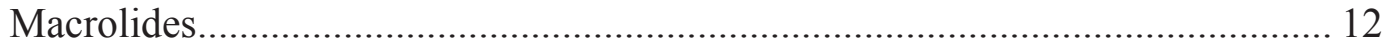

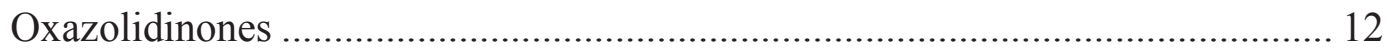

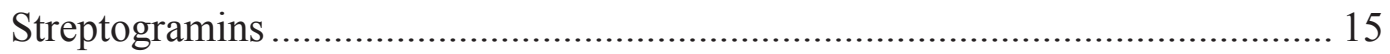

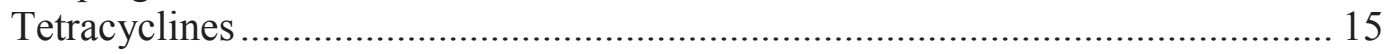

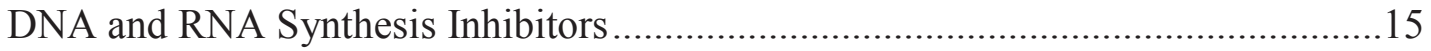

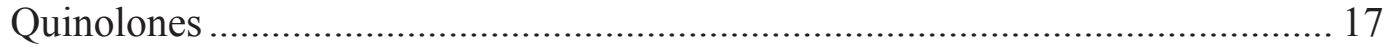

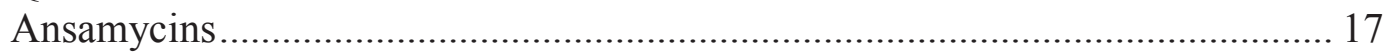

Reactive Species Generation and Targeting the Respiratory Chain .........................19

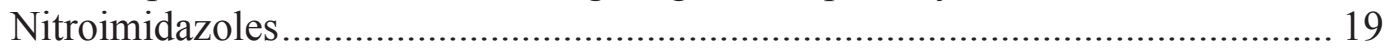

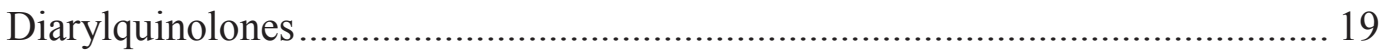

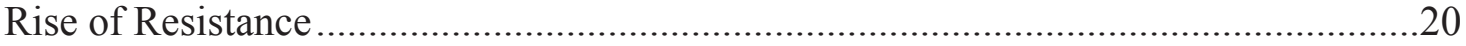

Causes and Common Types of Antibiotic Resistance ..............................................20

Decreasing cellular concentrations of drug................................................. 21

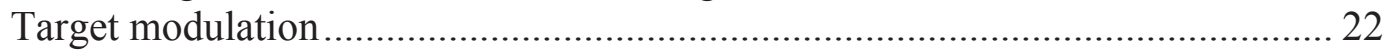

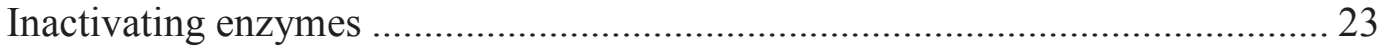

Discussion: Mitigating Resistance Through Drug Discovery ................................24

\section{CHAPTER 2. TARGETING THE CELL WALL OF MYCOBACTERIUM}

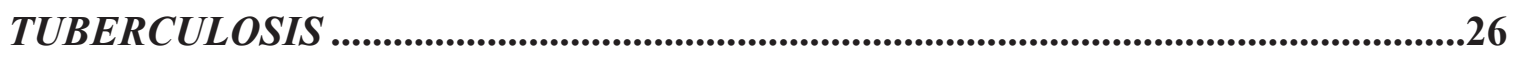

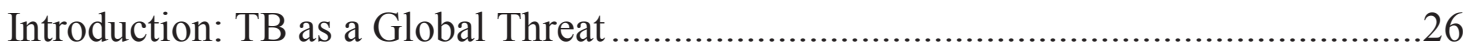

M.tb Life Cycle and Microenvironments ................................................................30

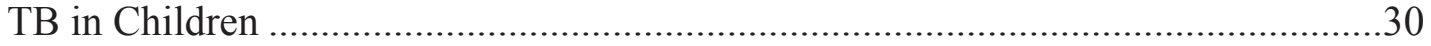

Agents to Treat TB and Known Issues in Adults and Children.....................................32

Adverse Effects of Front-line Agents .......................................................................34

Adverse Events to Second-Line Therapies ………………........................................36

Drug Resistance in Mtb .....................................................................................38

Notable Recent Advances in Drug Discovery Technologies for Mtb ........................38

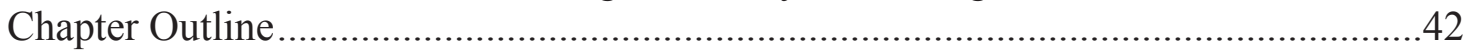

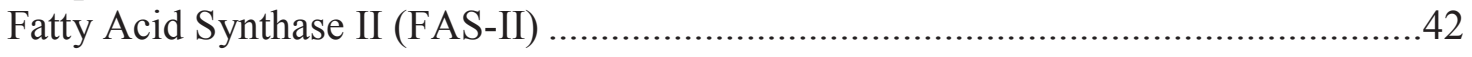


Introduction: HadAB-Known Inhibitors and Pharmacology .................................44

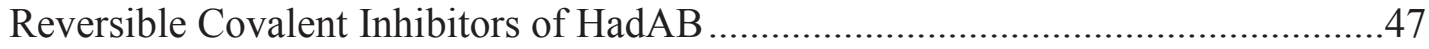

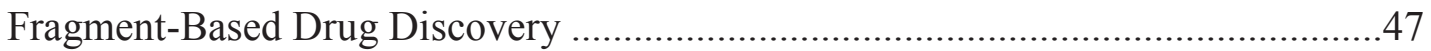

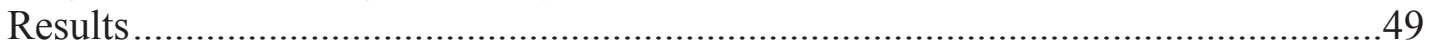

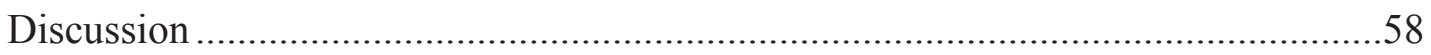

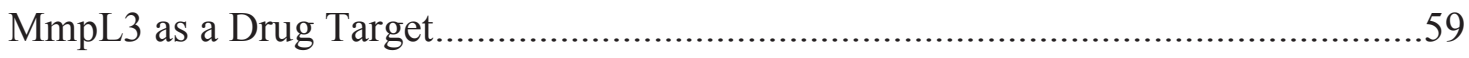

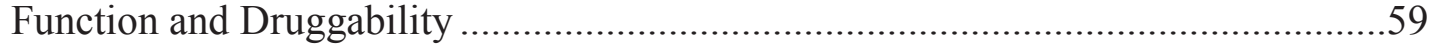

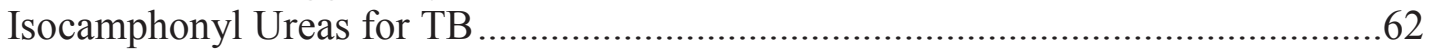

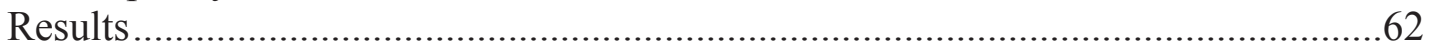

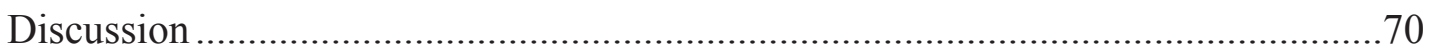

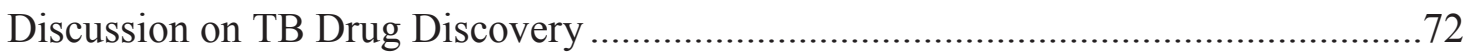

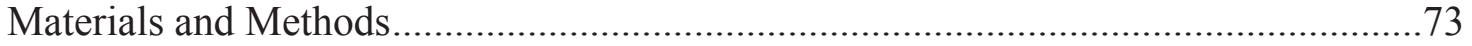

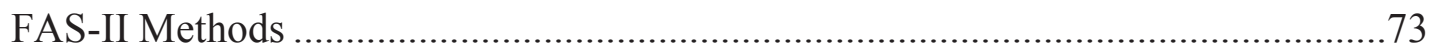

Protein purification ................................................................................. 73

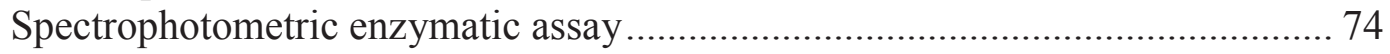

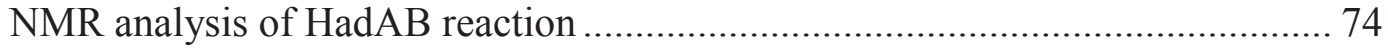

NMR analysis of 3216 inhibition of HadAB reaction ..................................... 74

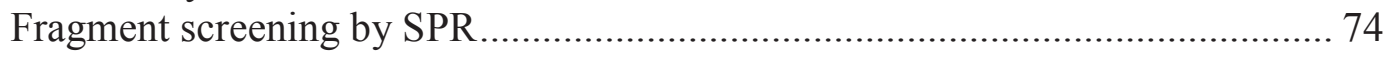

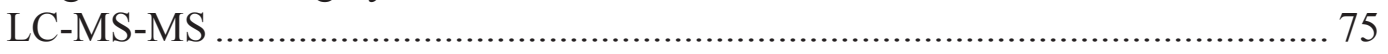

UPLC/MS/UV ................................................................................... 75

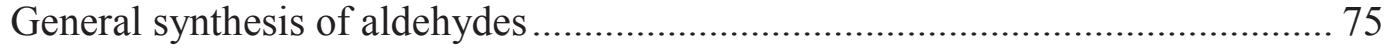

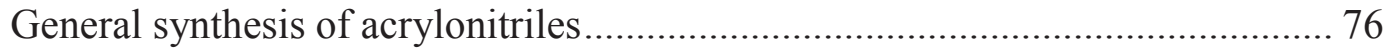

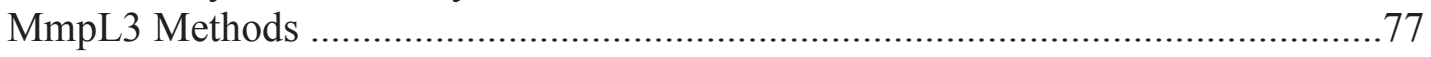

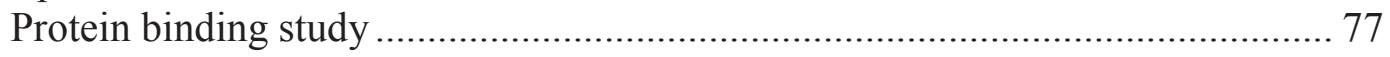

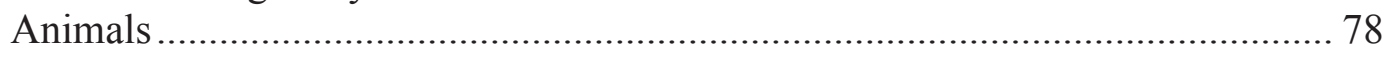

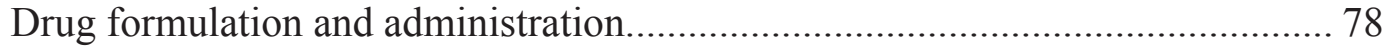

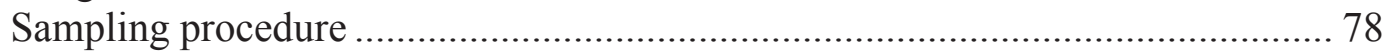

In-vivo pharmacokinetics....................................................................... 78

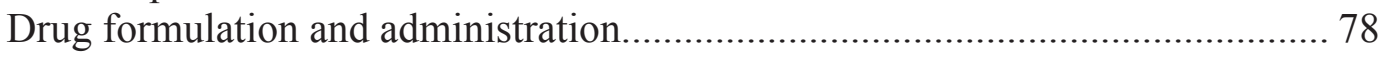

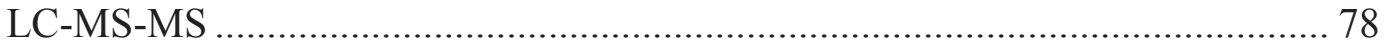

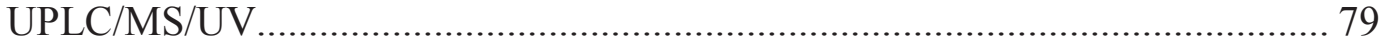

Synthesis of urea compounds: General method............................................ 79

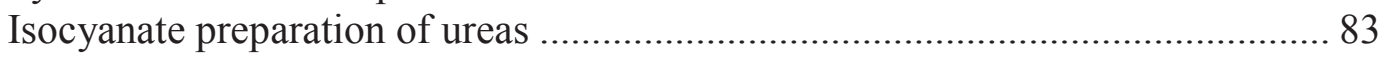

Preparation of methylene-spaced ureas .................................................... 83

\section{CHAPTER 3. DESIGN AND REPURPOSING OF ANTI-FOLATE
ANTIBIOTICS AND BIOACTIVES $\ldots \ldots \ldots \ldots \ldots \ldots \ldots \ldots \ldots \ldots \ldots \ldots \ldots \ldots \ldots \ldots \ldots \ldots \ldots \ldots \ldots \ldots \ldots \ldots \ldots \ldots \ldots \ldots \ldots \ldots \ldots \ldots \ldots \ldots$}

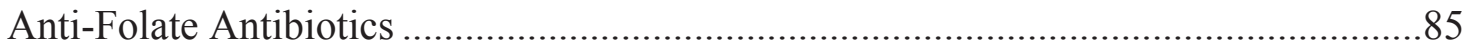

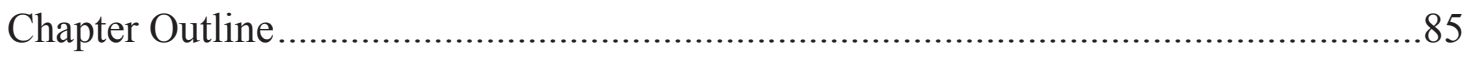

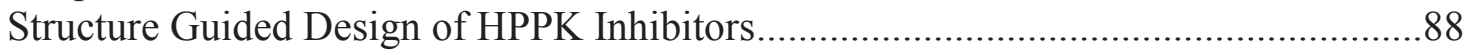

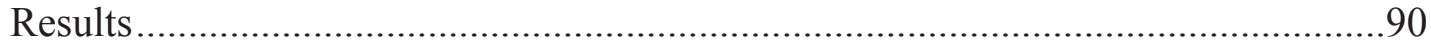

SAR Analysis of 8-Substituted-8-Thioguanine Derivatives ..................................92

Fragement Studies on the HPPK Pterin-Binding Pocket ........................................93

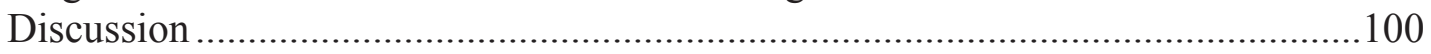

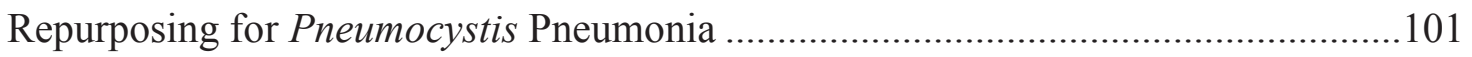

Introduction: Repurposing for New Leads Against Pneumocystis ......................... 102 


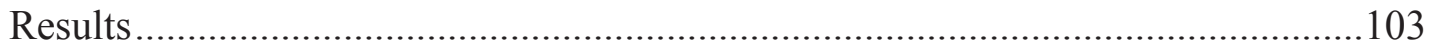

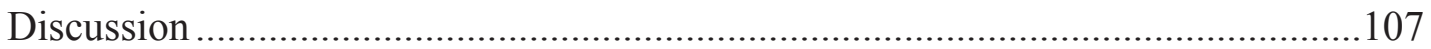

Discussion on Antibacterial Drug Discovery and Repurposing ...............................112

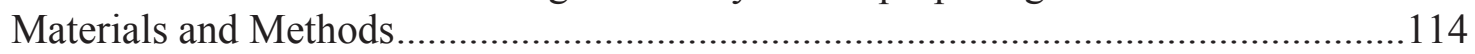

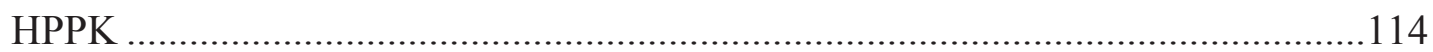

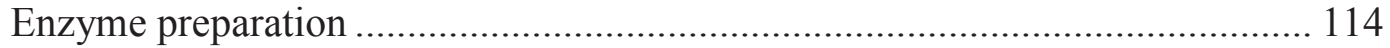

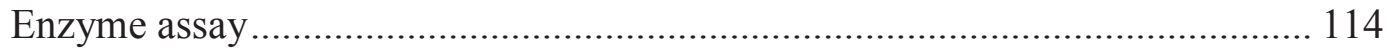

Biotinylation of HPPK ........................................................................... 114

Affinity analysis of compound binding by surface plasmon resonance ............ 114

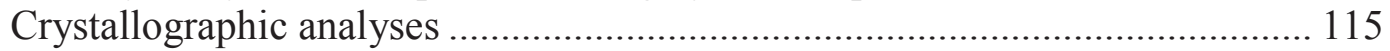

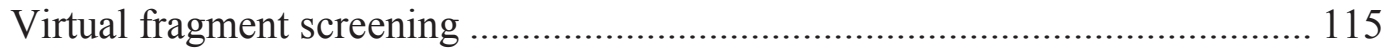

Synthesis of compounds: Materials and instrumentation ............................... 116

Synthesis of compounds: General procedure............................................... 116

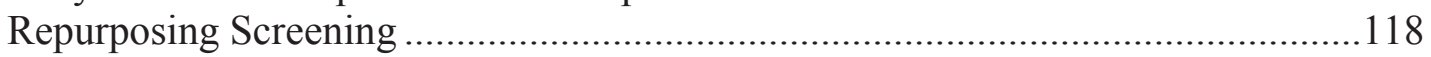

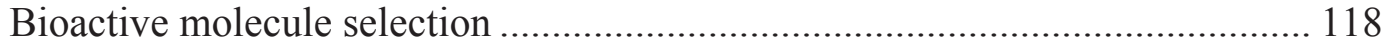

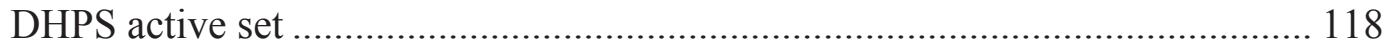

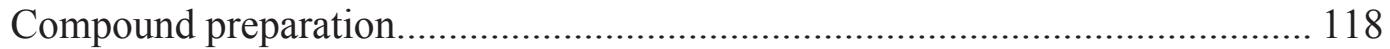

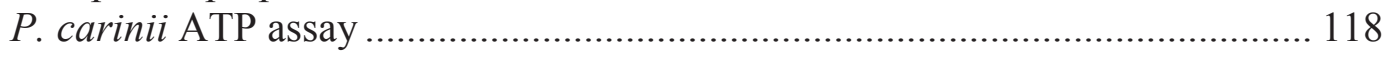

Calculations...................................................................................... 118

\section{CHAPTER 4. OVERALL DISCUSSION ON ANTIBIOTIC DRUG}

DISCOVERY: LESSONS AND EXPEIRENCES ..............................................120

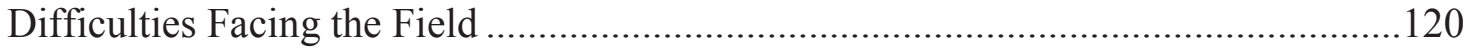

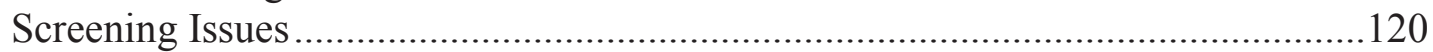

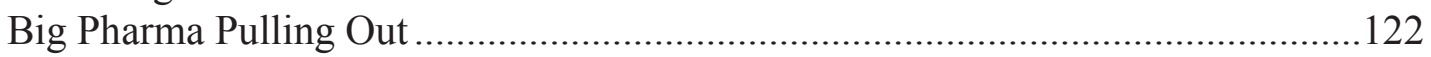

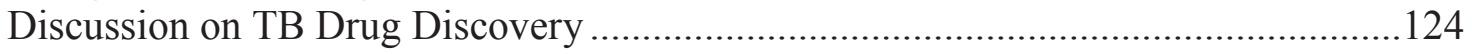

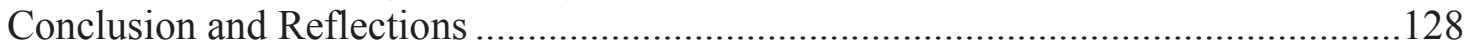

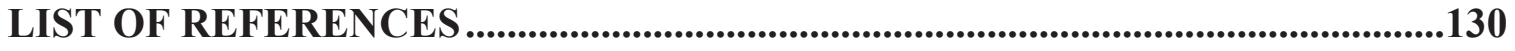

VITA 


\section{LIST OF TABLES}

Table 2-1. Groups of commonly administered agents for MDR and XDR infections and their noted adverse events in children

Table 2-2. Commonly associated drug resistance mechanisms to clinically relevant drugs to treat $\mathrm{Mtb}$

Table 2-3. All urea compounds with in vitro data................................................65

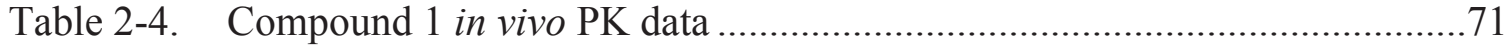

Table 3-1. All HPPK compounds with in vitro data ..............................................91

Table 3-2. HPPK inhibition data from 2D fragment screen......................................96

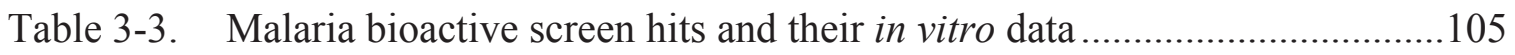

Table 3-4. DHPS inhibitors repurposing screen against Pneumocystis ......................109 


\section{LIST OF FIGURES}

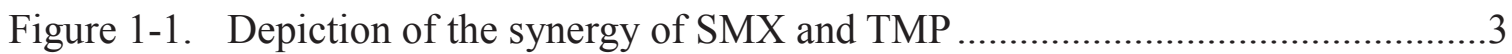

Figure 1-2. Various classes of $\beta$-lactam antibiotics and commonly used $\beta$-lactamase

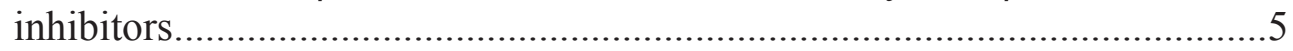

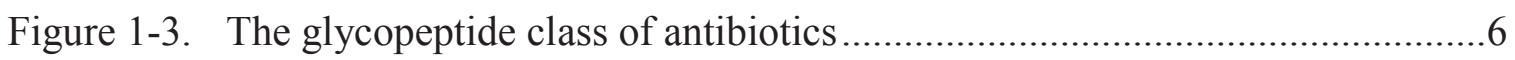

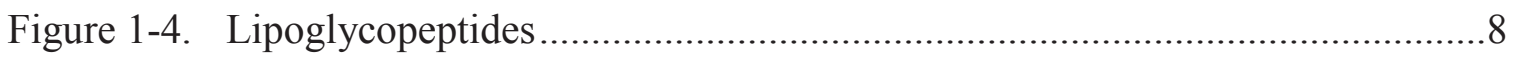

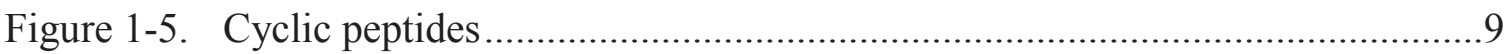

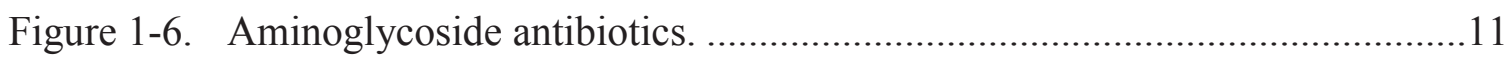

Figure 1-7. Chloramphenicol................................................................................11

Figure 1-8. The lincosamide class of bacterial protein synthesis inhibitors....................13

Figure 1-9. The macrolide class of antibiotics..........................................................13

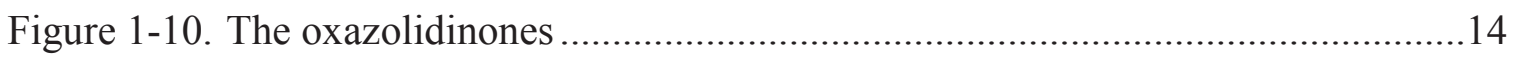

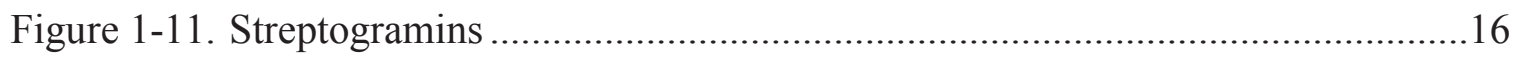

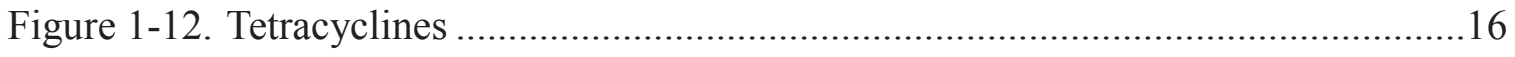

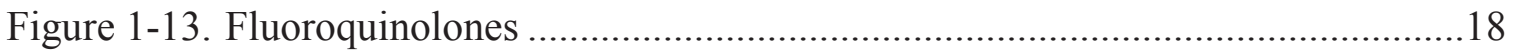

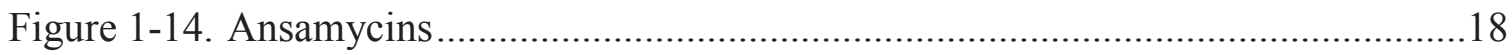

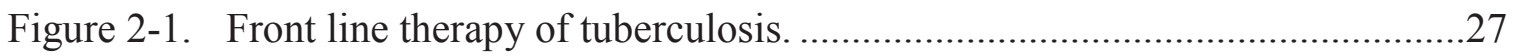

Figure 2-2. Bedaquiline and Delamanid...................................................................29

Figure 2-3. A simplified depiction of the Mtb lifecycle .....................................................31

Figure 2-4. General progression of agent addition to MDR-TB infection regimen.........33

Figure 2-5. The various environments a drug must thrive in to be an effective antitubercular agent

Figure 2-6. Mechanism of INH bioactivation by KatG and forming a covalent

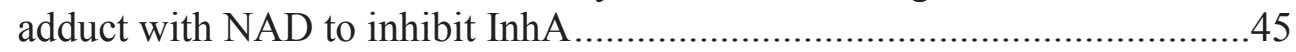

Figure 2-7. Mechanism of ISO's bioactivation to a sulfenic acid ....................................46 
Figure 2-8. Medicinal chemistry optimization of ibrutinib into a semi-covalent reversible acrylonitrile for residency time studies

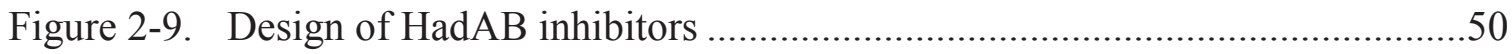

Figure 2-10. Rational development of more drug-like ISO mimics ................................50

Figure 2-11. Ribbon diagram of the HadAB heterodimer co-crystal structure with

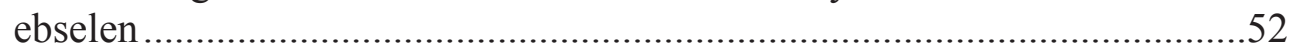

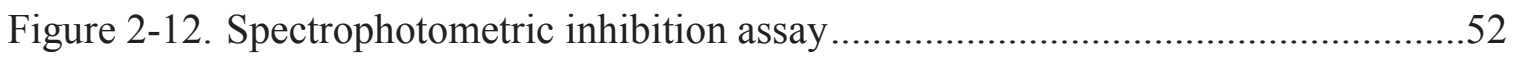

Figure 2-13. Four of the best acrylonitrile compounds ..........................................53

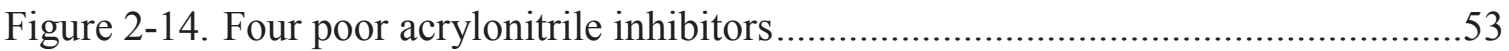

Figure 2-15. 3209 and 3188 regain activity by the deletion of one atom beyond the

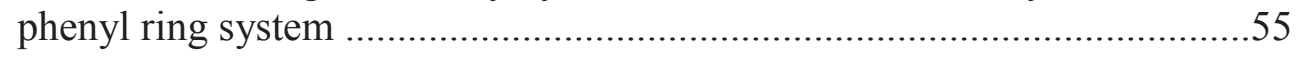

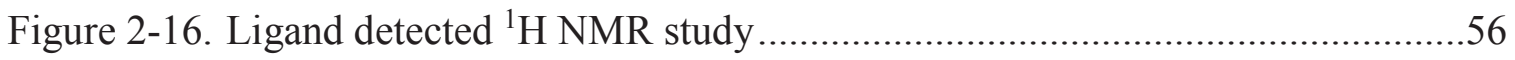

Figure 2-17. Representative fragment hits used to discuss the application of lipophilic ligand efficiency as a filter to select scaffolds to pursue going forwards. ...57

Figure 2-18. MmpL3 inhibitors .61

Figure 2-19. The initial HTS hit (1235) compared structurally to a known Mtb soluble epoxide hydrolase inhibitor.

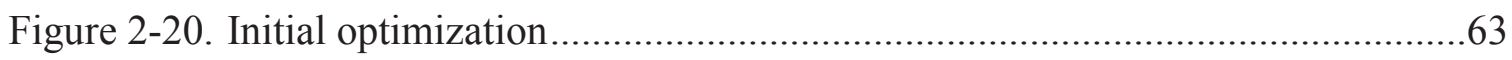

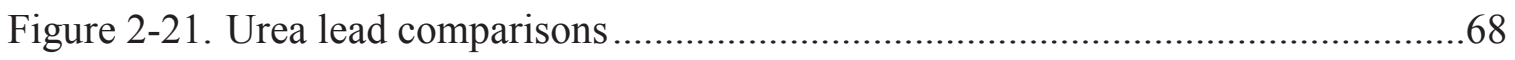

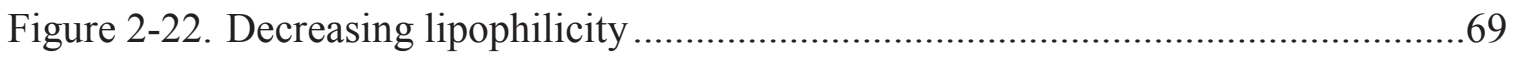

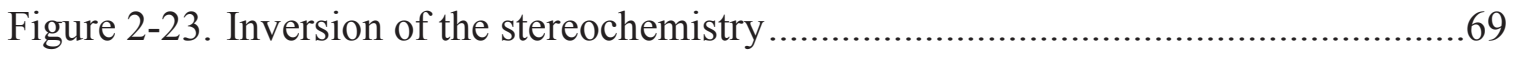

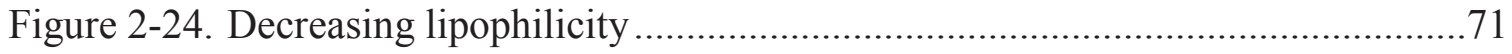

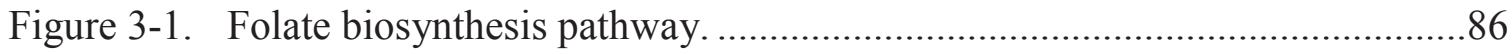

Figure 3-2. Illustration of SMX mimicking of pABA and the covalent modification

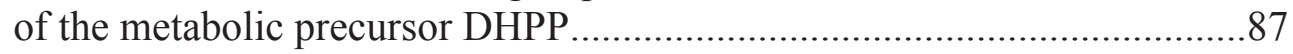

Figure 3-3. Crystal structure of 1 and 2 bound at the active site of HPPK ...................89

Figure 3-4. A-E. Abridged crystal structures of HPPK and inhibitors ..........................94

Figure 3-5. Crystal structure of HPPK ternary complex with compound 7 ..................95 
Figure 3-6. Two dimensional fragment screen

Figure 3-7. Biophysical fragment screen...................................................................99

Figure 3-8. 16 representative anti-malaria compounds of the 33 chemically diverse scaffolds selected for Pneumocystis screening..........................................104

Figure 3-9. Reaction scheme to generate analogs around one of the repurposing hits that demonstrates modularity and the ability to rapidly generate diverse

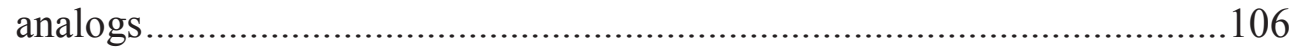

Figure 3-10. Example molecules included in the DHPS inhibitor screen for

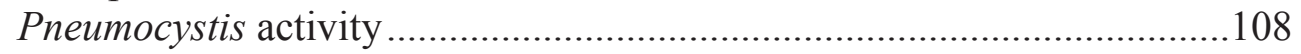

Figure 3-11. Structural comparision of 14207 to THPP ...................................................111

Figure 4-1. Physical chemical properties of currently used TB therapeutics vs newer

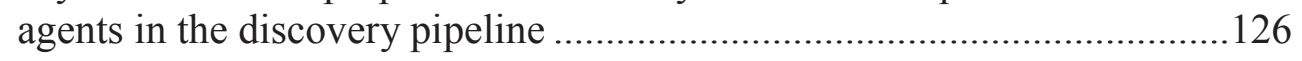




\section{CHAPTER 1. INTRODUCTION}

\section{Introduction of Antibacterial Agents}

The first synthetically derived chemical compound used in the treatment of bacterial infections, prontosil was synthesized as a dye in 1909 by a graduate student at the University of Vienna. In 1932 Bayer chemists discovered its usefulness in a mouse model of Streptococcus pyogenes and the field of medicine changed forever [1]. In the past 80 years morbidity associated with bacterial infections has reduced dramatically as a result of the bountiful discovery of novel chemical entities, through both synthetic design and naturally occurring efficacious compound identification. Penicillin is the classic example of naturally derived compounds with antibacterial properties, a $\beta$-lactam antibiotic that disrupts cell wall integrity through interrupted peptidoglycan biosynthesis. The "golden age" of antibiotic drug discovery has been described to have occurred in the decade between 1950 and 1960 as screening soil samples for antibacterial compounds secreted by soil residing bacteria such as Streptomyces griseus used to outcompete other bacteria [2]. These compounds had an intrinsic advantage over blind chemical screening in that they already had an essential antibacterial target and could effectively penetrate various bacterial outer cell environments. In this era one half of all clinically used antibiotics were discovered. These fresh discoveries could be classified in two main categories; bacteriostatic and bacteriocidal agents. Bacteriostatic agents are those that affect active replication such as through protein synthesis and limit growth and cell viability that allows host immune response to clear the infection. Bacteriocidal agents elicit cell death through the release of reactive species or through cell wall perturbations that cause catastrophic failure of the cell. Certain bacteriostatic agents can be cidal at high concentrations or in cases of synergistic combination therapies $[3,4]$. The discovery of antibiotics had unprecedented and multifaceted effects on the medical community as a whole. Organ transplants became possible with the use of immune suppression and prophylactic antibiotics to protect the patient. Aggressive chemotherapies for cancers were developed that had less concern for the patients succumbing to bacterial infections in the weakened state that followed. Surgeries that improved quality of life for the elderly such as joint replacement became viable. However the following decades leading up to the present have seen a marked decrease in new agents discovery, for a multitude of reasons to be discussed later. The need for unique agents, with novel mechanism of action is being driven by this lack of innovation as well as the emergence of drug resistant bacterial infections. We will first focus on existing drug classes of antibiotic compounds, and the pharmacological mechanism of action before going into common resistance mechanisms and the development of new agents.

\section{Inhibitors of the Folate Biosynthesis Pathway}

Folate is critical for the survivability of all living organisms as a co-factor in DNA synthesis via single carbon transfers in purine nucleotide biosynthesis [5]. Anti-folates

are selective to bacteria because they must produce their folates de novo while humans 
and other eukaryotes can obtain folates in their diet. There are two drug targets in this pathway, the dihydropteroate synthase (DHPS) and dihydrofolate reducatase (DHFR). Bactrim (Septra) is an orally bioavailable combination therapy used to treat methicillin resistant Staphylococcus aureus and a host of bacterial infections in a wide array of tissues. Bactrim is a 5:1 ratio of the DHPS inhibitor sulfamethoxazole (SMX) to the bacterial DHFR inhibitor trimethoprim (TMP) and is the classic example of antibacterial synergy (Figure 1-1).

\section{Sulfonamides}

Sulfonamides are synthetically derived mimics of para-amino benzoic acid (PABA), a metabolic precursor that is coupled with dihydropteroate pyrophosphate (DHPP) by the DHPS enzyme. There are a host of sulfonamide antibiotics that are generally well tolerated, but a small subset of people can have adverse events associated with sulfa drug allergies or hyper-sensitivity [6]. High instances of drug resistance have also been noted with sulfonamide antibiotics, so co-therapy with trimethoprim or another antibiotic is usually required. The most common mutations associated with sulfonamide resistance is an alteration in the PABA binding site that disfavors the larger substitutions [7].

\section{Trimethoprim}

Trimethoprim is a competitive inhibitor at the DHFR enzyme. It possesses robust synergy with DHPS inhibitors as they deplete levels of the endogenous DHFR ligand for TMP to compete against. Methotrexate, a drug used to treat various cancers and autoimmune diseases, is similarly a DHFR inhibitor. However TMP has a tremendous selectivity profile for the bacterial isoform of the enzyme that allows for a very wide therapeutic index with little toxicity associated with its use. Resistance to TMP is commonly associated with alterations in cell wall permeability and an overexpression or alteration of the DHFR enzyme [8].

\section{Cell Wall Biosynthesis Inhibitors}

One of the unique distinguishing characteristics used to classify various types of bacteria are the differences in their cell wall components. Gram staining is used to delineate the two large groups of bacteria into positive and negative classifications. Gram positive bacteria tend to have a thick layer of peptidoglycan, a polymer of sugars and amino acids that form intricate cross-linking leading to a thick outer wall that retains a deep violet dye [9]. Gram negative bacteria have a much thinner outer cell wall, but have a difficult to penetrate inner membrane that separates the periplasm and cytoplasm that disfavors the lipophilic compounds that are able to penetrate the peptidoglycan layer. The outer wall of gram negative bacteria has lipopolysaccharides, or endotoxins, that contribute to the toxicity of this group of bacteria. These bacteria tend to lose the violet stain and instead attach a softer pink dye [10]. Mycobacterium tuberculosis and other mycobacterium have an even more intricate outer layer, comparable to gram positive, but have a unique layer of long chain fatty acids called mycolic acids. In addition to this 


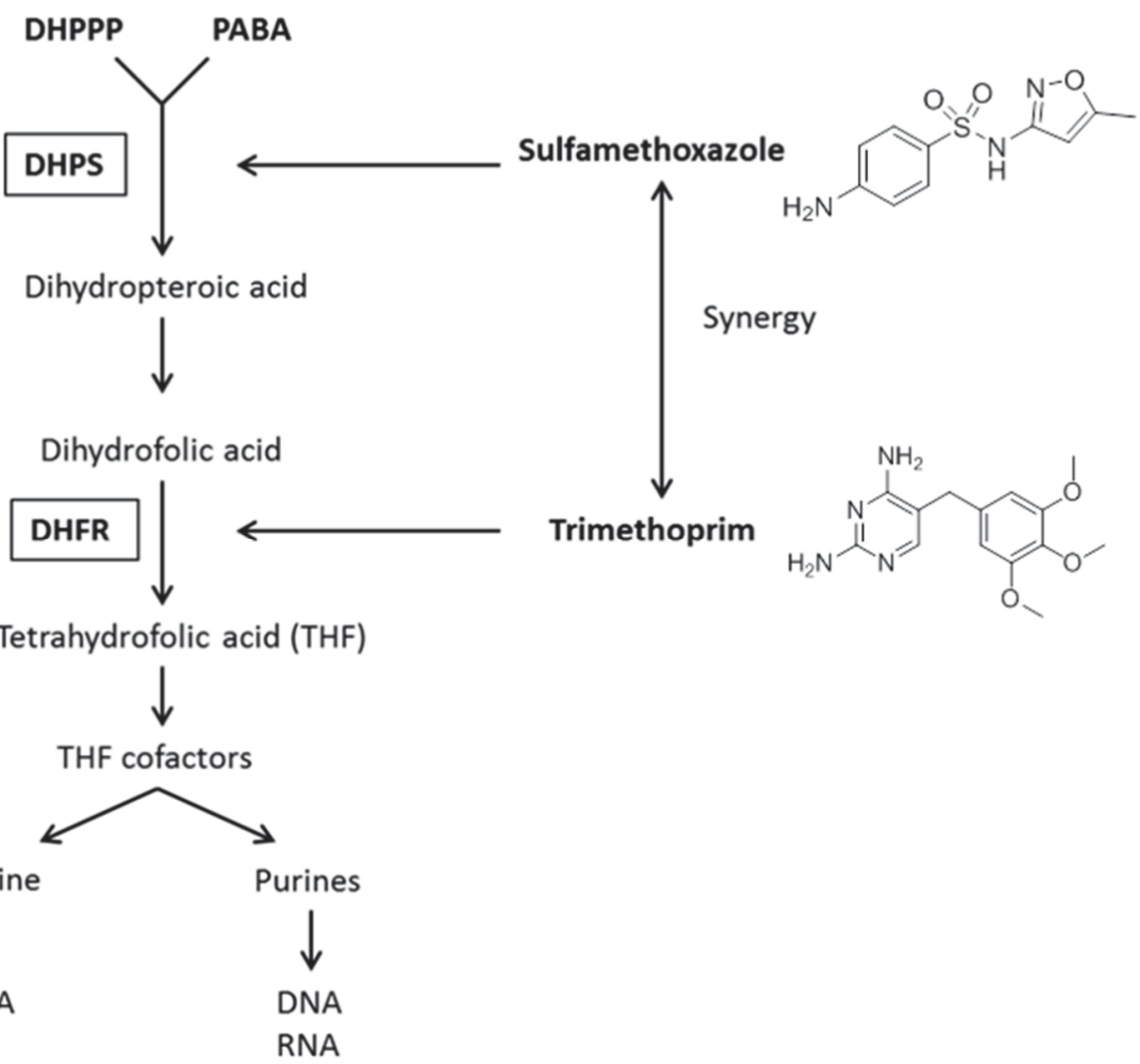

Figure 1-1. Depiction of the synergy of SMX and TMP

The classic example of antibiotic synergy 
waxy, difficult to penetrate outer layer of mycolic acids Mtb's contains lipoarabinomannan. This virulence factor is associated with inactivation of host macrophage immune response and protection against toxic oxidative radicals [11]. These various cell wall components have unique biosynthesis and transport pathways that offer scientists' ample opportunity to selectively target bacterial cells over eukaryotic as well as dial in selectivity for narrow spectrum of activity across bacterial species.

\section{$\beta$-lactams}

The most used and arguably important medical breakthrough in the past century was Ernst Chain, Howard Florey and Alexander Fleming's discovery of penicillin, and its therapeutic use in clearing bacterial infections. Penicillin is the first member of the $\beta$ lactam family of antibiotics that act by disrupting peptidoglycan crosslinking by covalently bonding to penicillin binding proteins (PBP) found at crosslink junctions. The series of naturally occurring penicillins, extracted from fungal secretions, spurned synthetic derivatives that have given rise to various classes of $\beta$-lactam antibiotics (Figure 1-2). $\beta$-lactamases are bacteria's first line of defense against most $\beta$-lactams and act by hydrolyzing the strained ring system that is the covalent attachment point before the drug can reach its target. Subsequent discoveries of later generations of $\beta$-lactam antibiotics such as cephalosporins and carbapenems are more naturally resistant to $\beta$ lactamases and were found to have a broader spectrum of activity. The next big breakthrough for this class came in 1976 with the discovery of $\beta$-lactamase inhibitors such as clavulanic acid [12]. These inhibitors are now commonly co-administered or even formulated with a $\beta$-lactam (Augmentin is amoxicillin and clavulanic acid). This series of antibiotics have broad spectrum of activity, including more recent applications in drug resistant tuberculosis therapy [13-15] and multiple dosage forms, making it the most versatile antibiotic class available.

$\beta$-lactamase inhibitors have had roughly four decades of clinical use, and their discovery certainly propelled the $\beta$-lactams to be perhaps the most clinically relevant class of antibiotics in history. They, however, are not immune to the development of resistance either. Over the years as new $\beta$-lactam antibiotics were discovered and used, there was a corresponding arms race with the bacteria producing even more potent and broadly acting $\beta$-lactamases. The inactivating enzymes are classified into four groups by the type of $\beta$-lactam they target (A,B,C and D) that will be discussed in more detail later. $\beta$-lactamase inhibitors work by mimicking the ring system that binds to the PBP and acting as a substrate for the $\beta$-lactamase enzymes. These substrates can either form a permanent covalent bond with the inactivating enzyme through a "suicide" attack or through slowly reversible, high affinity binding that sterically precludes hydrolysis [16]. The continued development of broad spectrum $\beta$-lactamase inhibitors is a major undertaking to combat drug resistance.

\section{Glycopeptides}

Glycopeptides such as vancomycin and teicoplanin (Figure 1-3) are antimicrobial heptapeptides secreted by actinomycete soil bacteria that inhibit peptidoglycan synthesis

by binding to the D-alanyl-D-alanine dipeptide site and by blocking transglycosylation 


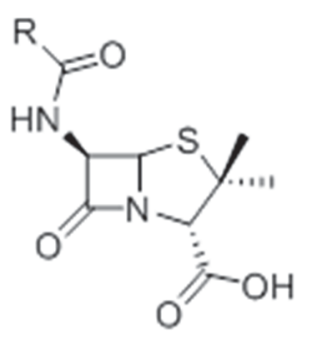

Penicillins<smiles>[R]C(=O)N[C@@H]1C(=O)N2C(C(=O)O)=C([X])CSC12</smiles>

Cephalosporins<smiles>O=C(O)C1C(=CCO)O[C@@H]2CC(=O)N12</smiles>

Clavulanic Acid<smiles>[R]C1=C(C(=O)O)N2C(=O)[C@H]([C@H](C)O)[C@H]2C1</smiles>

Carbapenems<smiles>[R]C(=O)N[C@]12O[C@@H](C)N(C1=O)C(C(=O)O)=C([X])CS2</smiles>

Cephamycins<smiles>CC1(C)C(C(=O)O)N2C(=O)C[C@H]2S1(=O)=O</smiles>

Sullbactam<smiles>[R]C1=C(C(=O)O)N2C(=O)C([R])C2S1</smiles>

$$
\text { Penems }
$$<smiles>[R]NC1CN(S(=O)(=O)O)C1=O</smiles>

Monobactams<smiles>O=C(O)C1[C@@H](Cn2ccnn2)S(=O)(=O)[C@H]2CC(=O)N12</smiles>

Tazobactam

Figure 1-2. Various classes of $\beta$-lactam antibiotics and commonly used $\beta$ lactamase inhibitors

Most commonly used and broadly acting antibiotics 

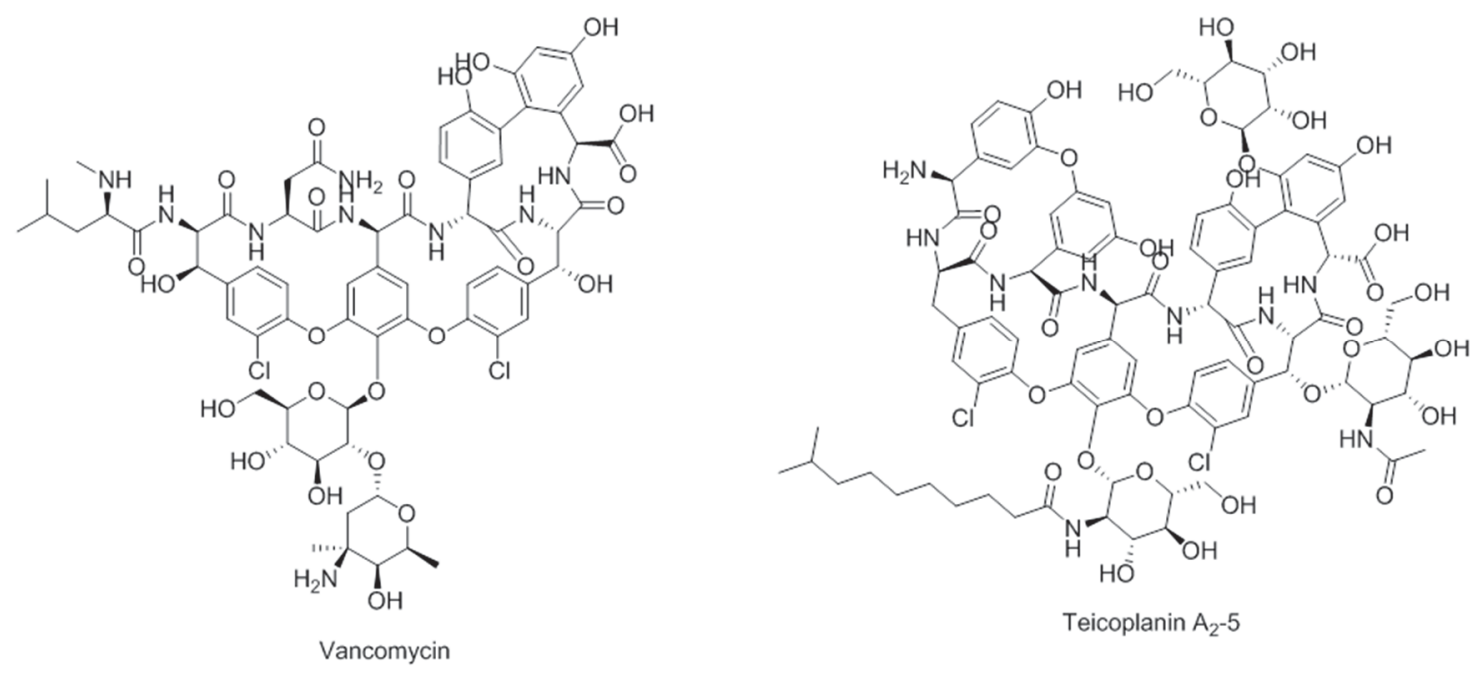

Figure 1-3. The glycopeptide class of antibiotics

Active against gram-positive bacteria 
and transpeptitdation [17]. These agents are used to treat serious Gram-positive infections such as Methicillin Resistant Staphalocacous aeruos (MRSA) and other genera resistant to $\beta$-lactam antibiotics, but are unable to penetrate across the inner membrane of Gramnegative. These large cyclic molecules tend to have poor drug-like properties and are limited to intravenous and topical administration, or oral administration for gastrointestinal tract infections. Drug resistance associated with these glycopeptides is very serious and treated with late stage $\beta$-lactams such as carbapenems, combinations like Septra (SMX:TMP), and cyclic depsipeptides like daptomycin. The common resistance mutation is an alteration the the vanA gene that results in the productions of VanA that allows for the peptidoglycan side chain to express a lower affinity substrate and crosslink like normal [18].

\section{Lipoglycopeptides}

Synthetic modifications that added lipophilic side chains to glycopeptides gave rise to a class of more metabolically stable and more potent of lipoglycopeptides [19]. They still contain the key heptapeptide core that allows for transglycosylation and transpeptidation inhibition. These agents include dalbavancin, oritavancin and telavancin (Figure 1-4). The increased stability allows for interesting pharmacokinetics; for example dalbavancin is dosed just twice, a week apart, and oritavancin is just one dose as a full course of therapy. The lipoglycopeptides are mainly used for skin and skin structure infections (SSSIs) of varying degrees of severity. Daptomycin (Figure 1-4) is a unique lipoglycopeptide in the sense that it interrupts cell membrane integrity, resulting in porous loss of ions and membrane depolarization in addition to inhibition of intercellular processes [20].

\section{Cyclic peptides}

A distinct class of peptide drugs is the cyclic peptides, which include homodetic (cyclosporine A) peptides, isopeptides (bacitracin) and depsipeptides (daptomycin) (Figure 1-5). These terminologies refer to the amount of non-amide linkages they contain in their main macrocycle. These cyclic peptides are more resistant to proteases than their glycopeptide and linear peptide relatives so they tend to at least partially survive the human digestive process required for oral bioavailability. Daptomycin is an excellent choice as a last resort antibiotic to treat extensively resistant Gram-positive infections. Resistance is commonly attributed to an increase in cell wall teichoic acid production and D-alanation, resulting in a thicker and more difficult to penetrate cell wall [21].

\section{Protein Synthesis Inhibitors}

Differences between the bacterial and human ribosome make another enticing drug target that was heavily exploited in the golden age of discovery. This broad classification of protein synthesis inhibitors includes the aminoglycosides, the tetracyclines, chloramphenicols, macrolides, oxazolidinones, lincosamides and 

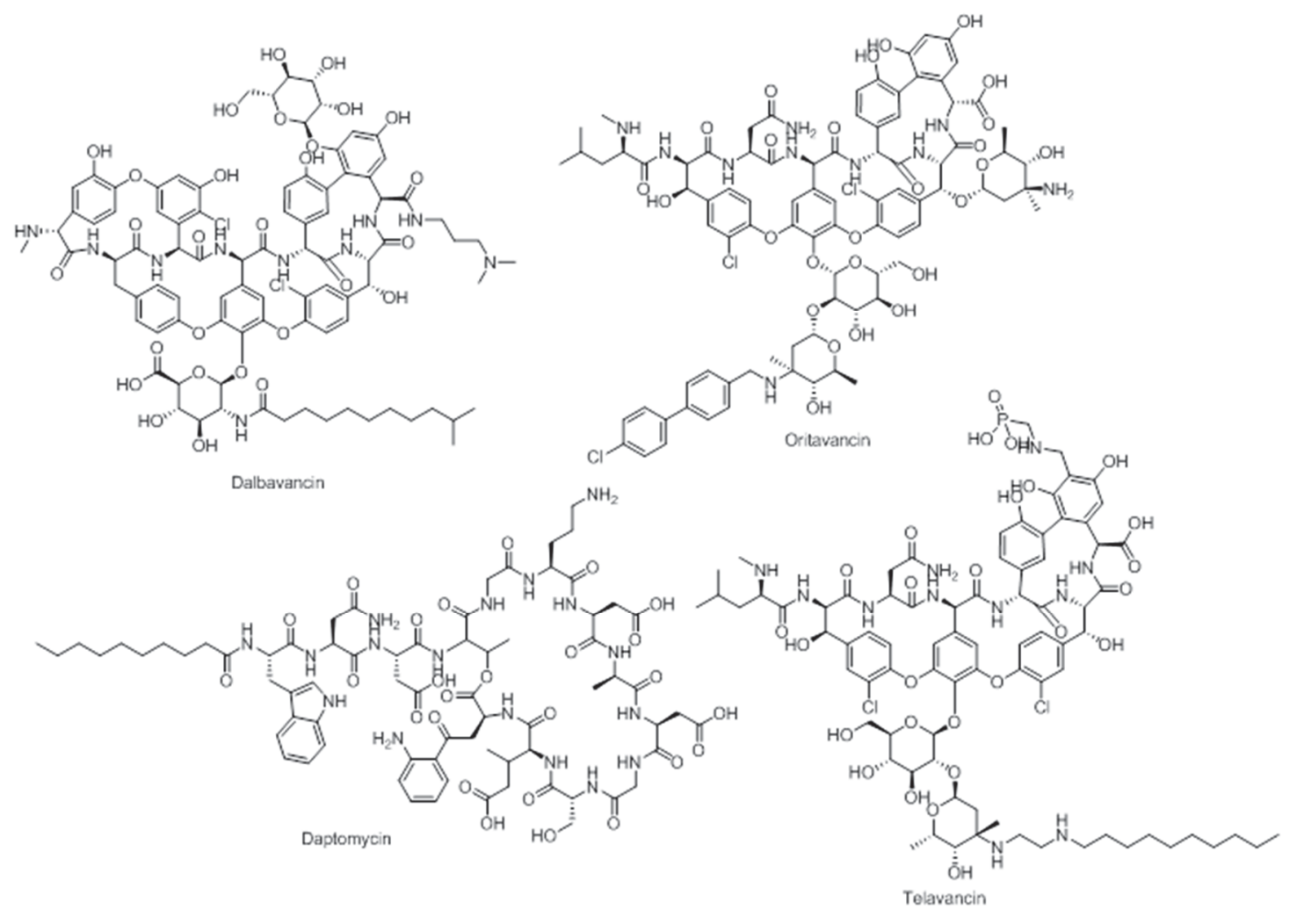

Figure 1-4. Lipoglycopeptides

Synthetic analogs of the glycopeptides with increased metabolic stability 

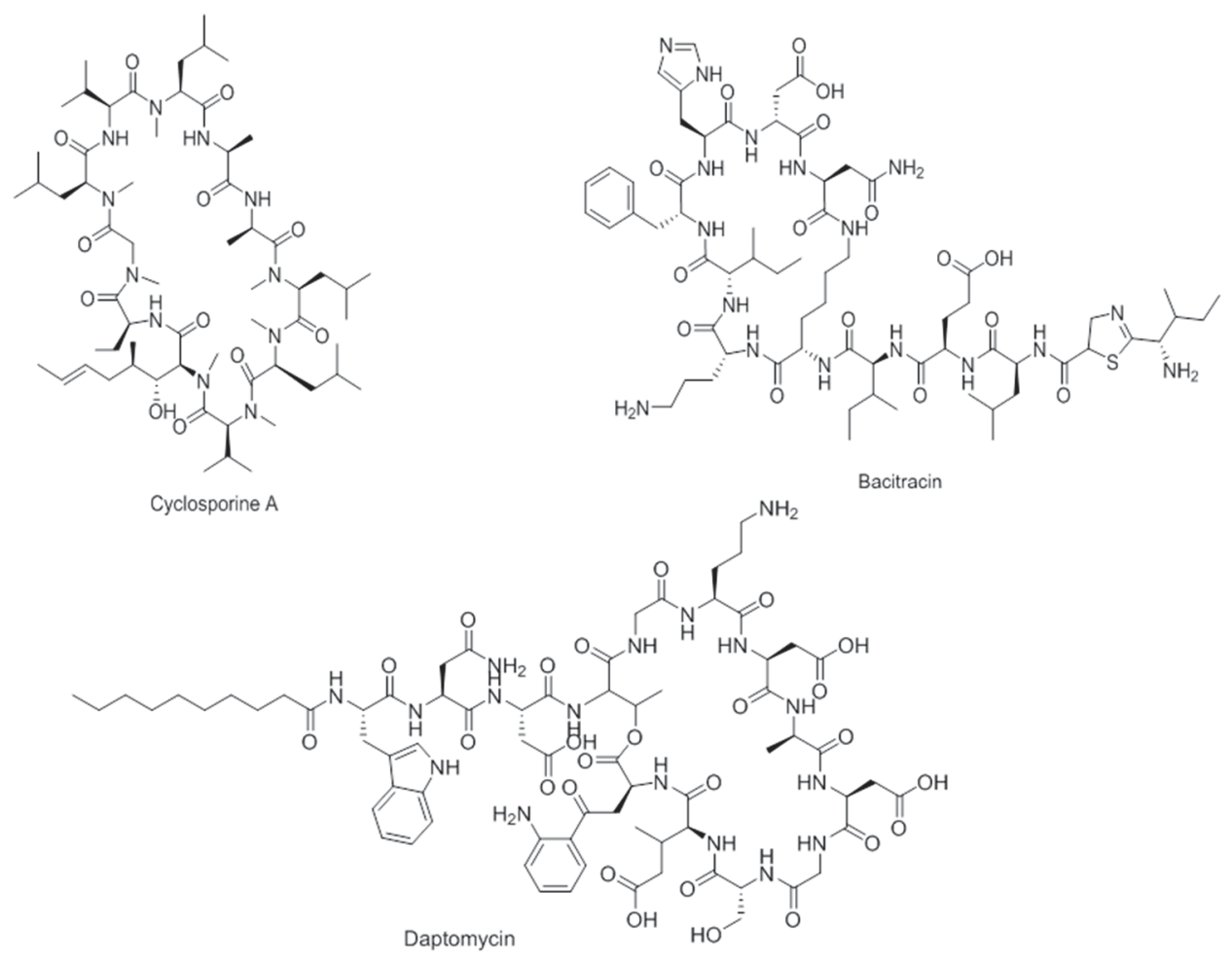

Figure 1-5. Cyclic peptides

Classified by the amount of non-amide bonds inside the ring. More stable in the stomach than glycopeptides allow for some oral bioavailability 
streptogramins. Protein synthesis inhibitors are primarily bacteriostatic with the aminoglycosides being the only primarily bacteriocidal agents. The bacterial ribosome is a complex of two ribonucleoprotein subunits (50S and 30S) that organize after the translocation of a charged tRNA and several initiation factors complex. The ribosome plays a crucial role in mRNA translation to form functional proteins [22]. mRNA translation occurs in three stages; initiation elongation and termination that involve the ribosome and cytoplasmic accessory factors. Drugs that target this mechanism are generally divided into which subunit of the ribosome they target, either the 50S or 30S. $50 \mathrm{~S}$ inhibitors typically act by blocking either the organization of the complex or by inhibiting translocation of the charged tRNA species which dramatically effects elongation of the growing peptide chain. 30Sribosimal inhibitors work by either blocking access of the aminoacyl-tRNAs to the ribosome [23] or by binding the 16S rRNA component of the $30 \mathrm{~S}$ subunit [24]. Some agents that bind to the $16 \mathrm{~S}$ component cause an alteration to the complex that promotes tRNA mismatching and a resultant mistranslated protein [25].

\section{Aminoglycosides}

The aminoglycoside antibiotic class is defined by a three dimensional system of aminated carbohydrate rings joined via glycosidic bonds (Figure 1-6). These are highly potent, broad spectrum antibiotics that possess many desirable drug-like physical chemical properties. Streptomycin was first identified in 1944 and many other similar compounds were found to be excreted from soil residing bacteria (kanamycin, gentamycin and tobramycin). This class possessed early Gram negative activity and led to the development of many semi-synthetic analogs in the 1970s (dibekacin, amikacin etc.), of which escaped early instances of resistance to the original aminoglycosides. The most common mutations associated with aminoglycoside resistance are binding site modulation of the $16 \mathrm{~S}$ component of the $30 \mathrm{~S}$ ribosomal subunit as well as an upregulation of deactivating aminoglycoside acetyltransferase [26, 27]. This is the only class of protein synthesis inhibitors that is associated with consistent bacteriocidal activity [28]. This is due to the mistranslation induced by the aminoglycoside interaction with the 16S rRNA that alters the complex formed between the mRNA and corresponding aminoacyl-tRNA [25]. This causes improper cell membrane integrity by the incorporation of mistranslated, and thus functionally attenuated proteins, into the wall allowing for an increase in aminoglycoside uptake [28].

\section{Chloramphenicol}

Chloramphenicol (Figure 1-7) is a bacteriostatic protein synthesis inhibitor. It acts by specifically binding to the A2451 and A2452 residues of the $23 \mathrm{~S}$ rRNA of the 50S ribosomal subunit that prevents peptide bond formation [29]. Chloramphenicol is noted with extensive adverse events and is subsequently rarely used, but its small molecular size and relative lipophillicity it penetrates the blood brain barrier effectively and is used in the treatment of abscesses in the brain as well as meningitis. Resistance associated to chloramphenicol is most commonly attributed to an altered 50S subunit as well as reduced membrane permeability and an increase in chloramphenicol acetyltransferase activity. 


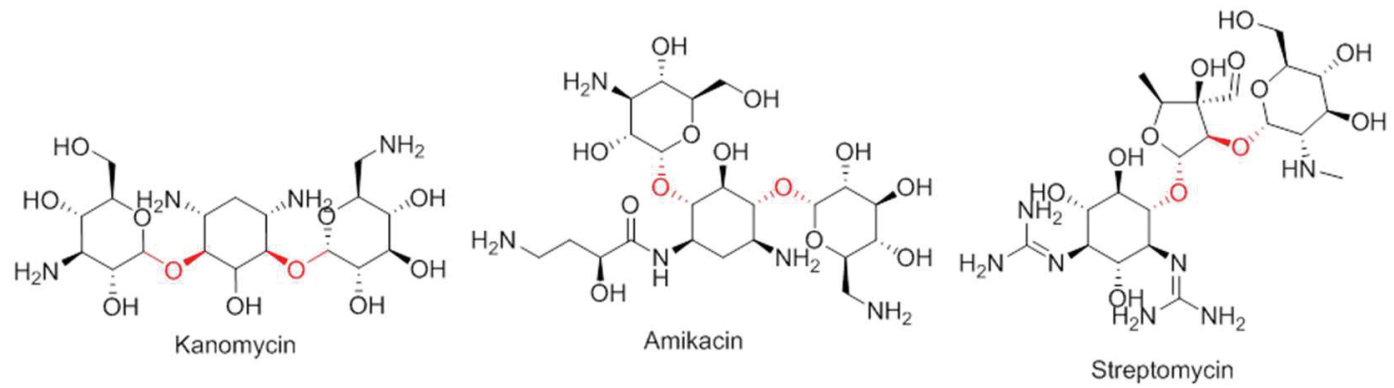

Figure 1-6. Aminoglycoside antibiotics.

In red depicts the glyocosidic bond.<smiles>O=C(N[C@@H](CO)C(O)c1ccc([N+](=O)[O-])cc1)C(Cl)Cl</smiles>

Figure 1-7. Chloramphenicol

A brain penetrating antibiotic that has noted toxicity issues 


\section{Lincosamides}

Lincosamides such as lincomycin and clindamycin (Figure 1-8) are bacteriostatic protein synthesis inhibitors. They bind to the $23 \mathrm{~S}$ portion of the $50 \mathrm{~S}$ subunit of the ribosome and cause a premature detachment of the peptide attached to the tRNA from the ribosomal complex [30]. Isolated from the soil bacteria Streptomyces lincolnensis and named after the city they were discovered in, Lincoln, NE, they possess a very similar pharmacodynamics profile with macrolides and are thus antagonistic when used together. Resistance emerges upon methylation of the $23 \mathrm{~S}$ portion that disfavors binding of lincosamides, macrolides and streptogramins.

\section{Macrolides}

The macrolide class of antibiotics is a well-populated class of polyketide macrocyclic natural products. The typical macrocyclic core is between 14 and 16 membered with various deoxy sugar rings attached (Figure 1-9). They are inhibitors of protein synthesis by blocking peptidyltransferase from coupling the growing peptide chain to the next amino acid and inducing a premature dissociation of the peptidyl-tRNA from the ribosome [30]. They bind to the 50S subunit at the same 23S rRNA as the chloramphenicols and lincosamides. Macrolides tend to accumulate in host leukocytes [31] and are thus transported to the site of infection by the immune system. This coupled with their bacteriostatic nature aids in macrolides in being efficient at dealing with infections used to treat gram positive and a few gram negative infections, and are commonly used in the case of penicillin allergies. The most common resistance mechanism associated with macrolides is the methylation of the $23 \mathrm{~S}$ ribosomal subunit that also results in cross resistance with lincosamides and streptogramins. The main macrolide antibiotics include 5 FDA approved agents, most notably azithromycin and clarithromycin (Figure 1-9). From here structural analogs have generated two subsequent generations of macrolides used to treat macrolide resistant infections. The ketolides (telithromycin, Figure 1-9) are particularly effective as they contain two ribosomal binding units, whereas the experimental fluoroketolides (solithromycin, Figure 1-9) have three such sites.

\section{Oxazolidinones}

Linezolid (Figure 1-10), the first-in-class oxazolidinone antibacterial agent, has demonstrated excellent clinical efficacy in treating drug-resistant gram positive pulmonary infections. Oxazolidinones are protein synthesis inhibitors that bind to the 50s ribosomal subunit of the $23 \mathrm{~S}$ rRNA. Linezolid use is generally restricted to less than 2 weeks, because the agent can cause myelotoxicity (as a result of mitochondrial protein synthesis inhibition), cytopenia, neuropathies, lactic acidosis, and rhabdomyolysis [32]. The class has grown to include more agents useful in drug resistant gram positive infections such as posizolid and tedizolid (Figure 1-10). Recently linezolid and oxazolidinones have received interest in treating drug resistant Mtb infections [33-37]. This is a promising new area for oxazolidinones that will be discussed in more detail later. 


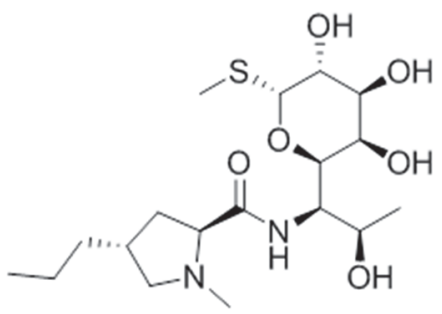

Lincomycin

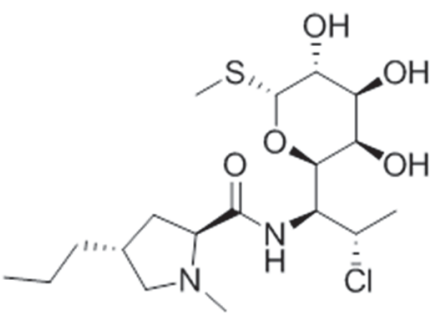

Clindamycin

Figure 1-8. The lincosamide class of bacterial protein synthesis inhibitors
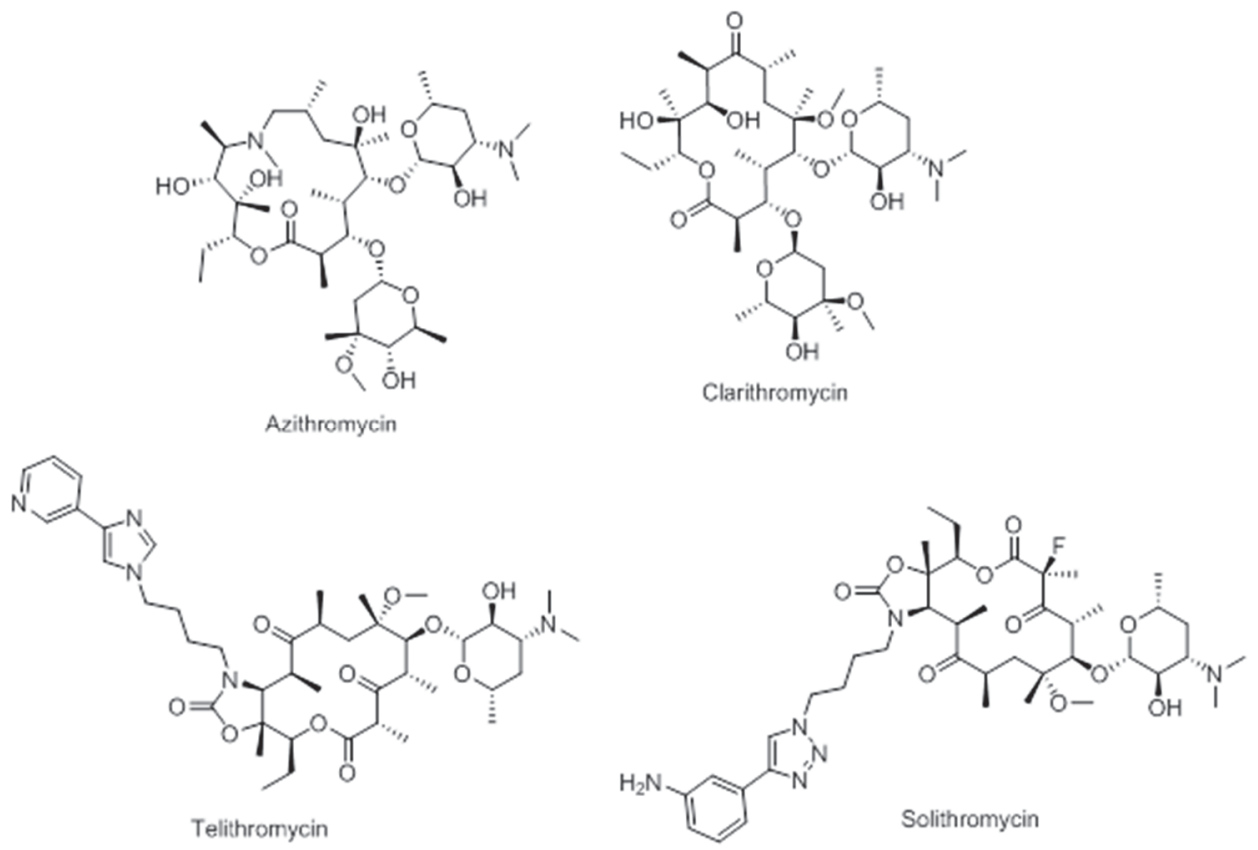

Figure 1-9. The macrolide class of antibiotics

Macrolides have been developed to incorporate more ribosomal binding units to mitigate resistance and increase potency 


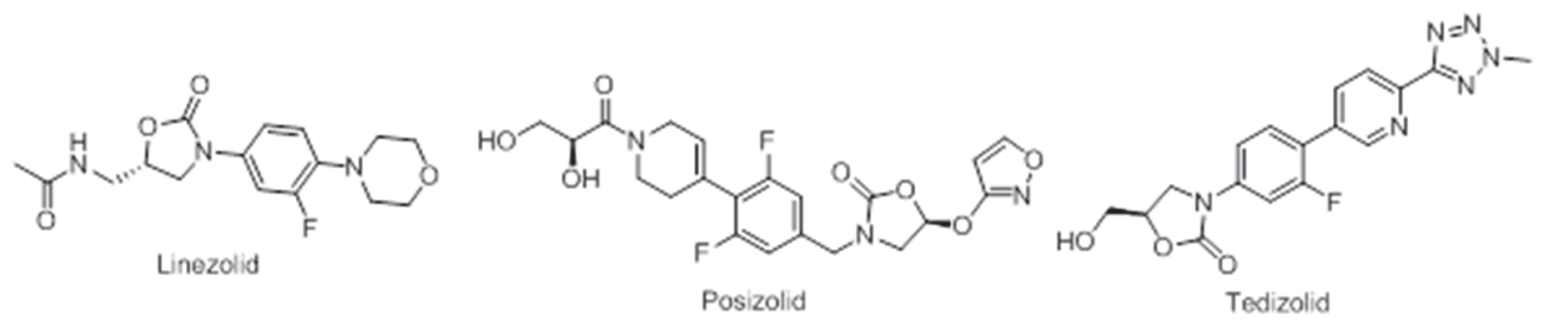

Figure 1-10. The oxazolidinones

The oxazolidinones have received renewed interest in the light of discovering great therapeutic potential 


\section{Streptogramins}

Streptogramins are combinations of two synergistic agents such as synercid; which is a combination of streptogramin A (dalfopristin, Figure 1-11) and streptogramin B (quinupristin, Figure 1-11) that are both bacteriostatic protein synthesis inhibitors when used separately but when co-administered they possess bacteriocidal activity. This combination therapy, Synercid, is highly efficacious in treating serious gram positive infections like vancomycin resistant Staphylococcus aureus (VRSA) and Enterococcus (VRE) and acts with polypharmacology. Dalfoprisin binds to the $30 \mathrm{~S}$ portion of the $70 \mathrm{~S}$ ribosome, altering its conformation which enhances quinupristins binding on the $50 \mathrm{~S}$ subunit [38].

\section{Tetracyclines}

Tetracyclines work by blocking access of the charged aminoacyl-tRNAs to the ribosomal acceptor (A) site of the 30S subunit of the ribosome. The naturally occurring tetracyclines (oxytetracycline, chlortetracycline and demethylchlortetracycline) have been synthetically modified to give rise to the more commonly used semisynthetic class (minocycline, doxycycline etc, Figure 1-12). Tetracyclines are broad spectrum acting agents, having activity against both gram positive and negative organisms as well as more atypical pathogens such as chlamydiae, mycoplasmas and protozoa. This in conjunction with their relative lack of adverse events in mammals have led to their common use in many human and animal infections, including prophylactically in the prevention of malaria spread [23]. Important to the discussion of antibiotic resistance is the fact that tetracyclines have traditionally been used in subtherapeutic doses in animal feeds as growth promoters. The common resistance adaptation many bacteria employ against tetracyclines is the acquisition of active efflux pumps [23]. Resistance is generally slow emerging to this class, but the widespread use of subtherapuetic doses has led to a plethora of bacterial species whom have acquired resistance. Researchers have employed more synthetic modulation techniques to develop the glycylcyclines, led by tigecycline (Figure 1-12). This distinct class of tetracyclines have incorporated a bulky substituent that aids the molecule to be disfavored for efflux [39]. This sort of synthetic modification with drug resistance in mind will be critical in the reactivating drug classes with noted drug resistance and has already began with the development of novel spectinomycins for the treatment of tuberculosis and gram negative pathogens [40].

\section{DNA and RNA Synthesis Inhibitors}

Selective inhibition of bacterial DNA or RNA synthesis has been an important way of inducing bacterial cell death since the discovery of quinolones and rifamycins. Drugs that work by blocking DNA synthesis work on either the DNA gyrase (topoisomerase II) or topoisomerase IV. While functionally similar it seems that targeting one versus the other gives rise to gram species selectivity, for example targeting topoisomerase IV seems to illicit a greater response in gram positive bacteria while targeting DNA gyrase effects gram negatives more [41]. Inhibition of RNA synthesis has 

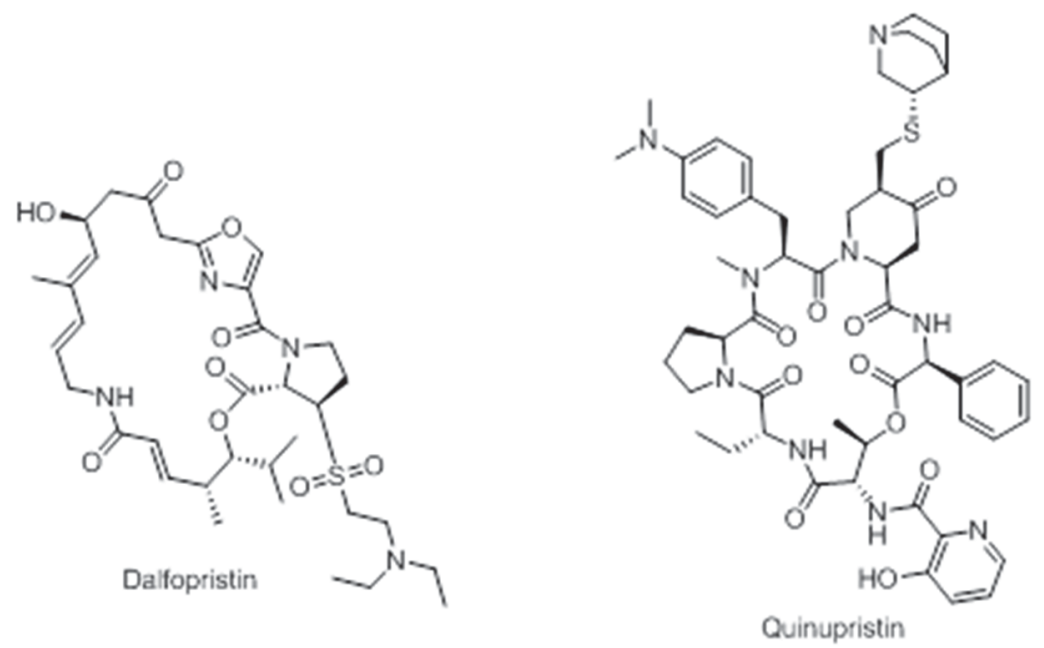

Figure 1-11. Streptogramins

The synergistic combo of dalfopristin (streptogramin A) and quinupristin (streptogramin B) make up the bacteriocidal agent

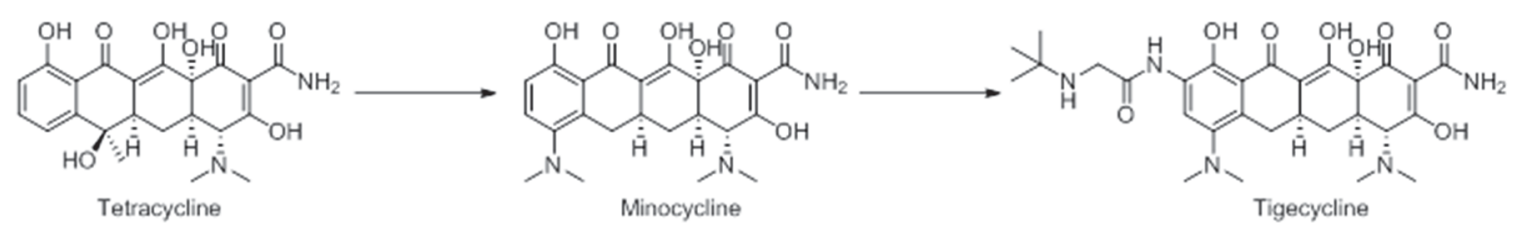

Figure 1-12. Tetracyclines

Semi-synthetic optimization of tetracyclines has produced an efflux resistant molecule in tigecycline 
a devastating effect on nucleic acid metabolism and is achieved by rifamycins by inhibition of the RNA polymerase.

\section{Quinolones}

Quinolones and their more clinically relevant fluoroquinolone derivatives work on inhibition of DNA gyrase and topoisomerase IV. This inhibition results in an interference in the maintanence of chromosomal topography by preventing proper DNA supercoiling. This is an attractive target as eukaryotic cells don't require DNA supercoiling. They work by trapping the enzyme at the DNA cleavage site and not allowing for DNA strand reformation, resulting in double strand breakage and covalently attached complexes of quinolone-topisomerase-DNA [42]. Fluoroquinolones are the more clinically favored class and generally have a fluorine at the C-6 or C-7 position of the central ring (Figure 1-13). They are broad spectrum acting agents with a few later generation iterations being used in M.tb optimized regiments for MDR-TB and pediatric TB $[43,44]$. Later generation fluoroquinolones such as Moxifloxacin and Gatifloxacin (Figure 1-13) have been used for gram negative respiratory infections, but have noted issues of toxicity such as dysglycemia and spontaneous tendon rupturing that limit their use as well as risk of QT interval prolongation [45]. The most common resistance mutations occur at gyrA and gyrB causing an alteration to DNA Gyrase A/B binding site (later generations not always cross resistant with first generations) and increased ABCtype efflux pump expression [27].

\section{Ansamycins}

Ansamycins, otherwise known as the rifamycins, inhibit RNA synthesis and induce cell death by binding to the rpoB subunit of a DNA bound RNA polymerase and block transcription [42]. Anamycins are easily recognizable by their atypical aromatic bridge in their macrocycle, the basket that aliphatic portion of the ring is the "handle" for (ansa $=$ handle in latin). Rifamycins were originally isolated from the soil bacteria Streptomyces mediterranei (since renamed several times to Amycolatopsis rifamycinica) in the 1950s, semisynthetic efforts yielded molecules like rifampicin (RIF, Figure 1-14). Rifamycins are considered bacteriocidal against gram positive and M.tb but bacteriostatic against gram negative. Resistance emergence is common and rapid [42] so rifampycins are usually used in combination therapy such as the front-line cocktail for tuberculosis, rifafour. RIF is considered the most important member of anti-tubercular regimens and multidrug-resistance is associated with resistance to RIF and isoniazid (INH). Rifamycins are also noted as potent drug metabolizing and transport enzyme modulators [43]. This is most important in the increasingly common instance of $M$.tb co-infection with the human immunodeficiency virus (HIV) [44]. RIF is a potent cytochrome p450 (CYP) inducer, the main metabolizing enzymes for early highly active antiretroviral therapy (HAART) agents such as zidovudine and efavirenz $[45,46]$. The most commonly associated resistance occurs at the $r p o B$ gene which causes a conformational change at the RIF binding site of the RNA polymerase [42]. 

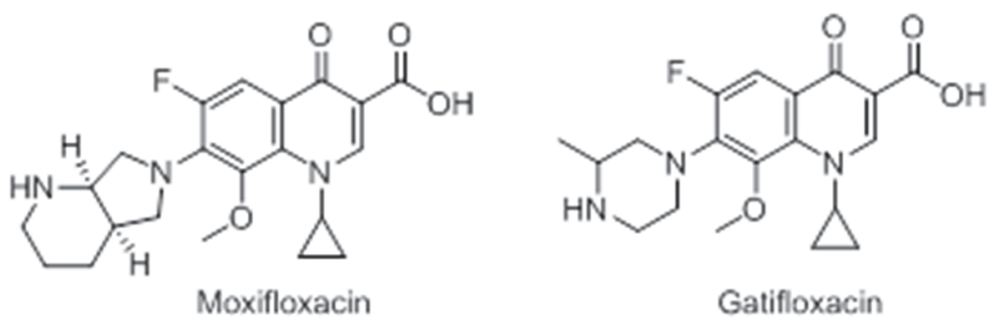

Figure 1-13. Fluoroquinolones

Act as DNA gyrase and Topoisomerase IV inhibitors that prevent DNA supercoiling. They are efficacious against gram negative

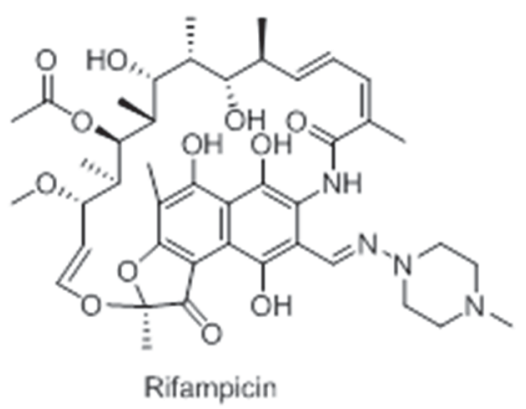

Figure 1-14. Ansamycins

Perhaps the most important drug for the treatment of tuberculosis, rifampicin is also highly active in gram-positive bacteria but only bacteriostatic in gram-negative 


\section{Reactive Species Generation and Targeting the Respiratory Chain}

It is speculated that all bacteriocidal agents elicit their cidal activity from a common production of reactive oxygen species (ROS) caused by bacterial stress responses [47]. These lethal species formations are spurned by an increase in the respiratory and TCA cycles caused by stress responses systems such as envelope stress (Cpx) and redox-response (Arc) [48]. This alteration to cell metabolism is important in destabilizing iron-sulfur clusters resulting in an increase in the Fenton reaction that generates hydroxyl radicals $[28,47]$. This section will discuss a few series of new compounds that both release their own reactive oxygen species and those act on the respiratory chain to elicit bacteriocidal effects.

\section{Nitroimidazoles}

Resistance to nitroimidazoles is quite slow developing and relatively rare compared to many other classes of antimicrobial agents [49]. They possess a vast range of activity, active across both gram stains of bacteria as well as protozoa, solid tumors and M.tb. Metronidazole is perhaps the most famous of these antimicrobials; it has been in use since the 1960s to treat many anaerobic infections as the reduction required for activation only occurs in hypoxic environments. This activation is a reduction of the nitro group that results in a short lived nitro radical anion that can oxidize DNA causing strand breakage and cell death [49].

\section{Diarylquinolones}

The diarylquinolones are the most advanced series of novel antitubercular drugs. The recently approved bedaquiline falls under this classification. Bedaquiline is highly selective and specifically inhibits essential ATP-synthase activity in replicating and dormant mycobacteria but not in other prokaryotic or eukaryotic cells [50, 51]. Through the growth of drug-resistant mutants, it was discovered that the rotor ring of the organism's $\mathrm{F}_{0} \mathrm{~F}_{1}$ ATP synthase was the target, specifically binding to the c subunit [52]. Mtb survives in a nonreplicating state using a pool of ATP that is used to maintain an energized membrane produced by the $\mathrm{F}_{0} \mathrm{~F}_{1} \mathrm{ATP}$ synthase [53]. This makes bedaquiline a prime weapon for killing the latent Mtb subpopulation. With a distinct mechanism of action from RIF and INH, bedaquiline is an effective addition to an MDR-TB regimen. Discovered during a phenotypic screen performed by Janssen, bedaquiline shows excellent activity against drug-sensitive and drug-resistant isolates, with a minimum inhibitory concentration (MIC) of $0.03 \mu \mathrm{g} / \mathrm{mL}$ for drug-sensitive Mtb and $0.12 \mu \mathrm{g} / \mathrm{mL}$ for drug-resistant strains, as well as outstanding activity in in vivo murine models. However, bedaquiline's efficacy in mice has not been as pronounced in humans, presumably due to limited distribution within human granulomas. Bedaquiline is a large lipophilic drug that is subject to CYP3A4 metabolism and has been noted to have PK drug-drug interactions with the CYP3A4 inducer RIF, which decreases its activity [54]. Bedaquiline has been associated with QT-prolongation and must be carefully combined with other drugs that share this risk, including fluoroquinolones, macrolides, clofazimine, or drugs that inhibit CYP3A4, which may increase its exposure [55]. Thus, efforts are ongoing to develop second-generation diarylquinolones that will improve the drug's physiochemical 
properties and remove its cardiac liability. This effort has been recently aided by the cocrystal structure of bedaquiline bound to its active site in the ATP synthase, which should enable structure-guided design of more potent and less toxic analogs [56].

\section{Rise of Resistance}

During the years immediately following the "Golden" age of antibiotic drug discovery scientists felt the discovery pipeline would never dry up. A quote attributed to then United States Surgeon General Dr. William Stewart claimed "It is time to close the book on infectious diseases, and declare the war against pestilence won". However, almost as soon as the first antibiotics were administered, resistance began to emerge in the forms of genetic mutations to the bacteria that allowed them to survive chemotherapeutic intervention. This phenomenon was predicted by Sir Alexander Fleming, the man responsible for the discovery of penicillin, as a consequence of improper and over use of antibacterial compounds [57]. Within a few years of the widespread manufacturing and use of penicillins in non-WWII situations, over $50 \%$ of isolated bacterial infections were no longer susceptible to penicillin. In current days drug resistant infections have become a source of great concern. Hospital acquired infections are problematic, both in increased mortality rates and increased cost burdens. MRSA infections are $64 \%$ more likely to be lethal than their drug susceptible counterparts, and are expected to cost millions every year [58]. Multiple other pathogens have developed worrisome resistance including but not limited to M.tb, Acinetobacter, Pseudomonas, Enterococcus and Pneumococcus. These include bacteria that are resistant to one antimicrobial agent, those resistant to two or more (multidrug-resistant, MDR) and those that are extensively (XDR) or totally drug resistant (TDR). The rapid spread of resistance is generally attributed to inappropriate use of these agents, as well as natural evolutionary pressure for these organisms to overcome chemotherapeutic intervention [57]. We will discuss some of the specific causes of resistance emergence as well as commonly occurring mechanisms, most of whom have been discussed in detail in the previous section. Finally we will close this section with recent discovery efforts in the field, some success and some lessons learned going forward.

\section{Causes and Common Types of Antibiotic Resistance}

It was reported in 2010 that the on average the average US citizen consumed 22 units (unit meaning one dose) of antibiotics, a number that varied greatly by state, with those in the mid-south being the highest consumers [59]. This combined with the estimation that $30-50 \%$ of antibiotic therapies are either misdiagnosed, use the wrong drug or are not completed to the proper duration makes for a perfect breeding ground for drug resistance to cultivate $[57,60]$. In addition to improper dosing, agriculture across the world has been supplementing animal feed with sub-therapeutic concentrations of antibiotics, albeit usually those not used in humans. This use is to promote health and growth in animals and prevent disease, but allows bacteria to experience a drug at sublethal concentrations or improper medication for a particular organism. This gives the 
surviving bacteria a chance to confer resistance and propagate drug resistant strains. This now resistant organism may eventually drop this resistant mutation, but if the fitness cost associated with this new found resilience is not too high than it can propagate unabated [61].

In 2014 it was estimated that more than 2 million people in the United States were infected with drug resistant bacteria, directly resulting in 23,000 deaths [62]. Bacteria can be intrinsically resistant to drug intervention, such as Pseudomonas' intrinsic resistance to the antiseptic triclosan. This has been found to be because Pseudomonas encodes an extra enoyl-ACP reductase enzyme, $f a b V$, that is insensitive to that drugs primary mechanism of action [63]. Many gram negative organisms are intrinsically resistant to agents potent against gram positives due to their differences in cell wall composition, daptomycin and vancomycin being the best examples of this phenomenon. Understanding intrinsic resistance factors can be very important in identifying proper combination therapies to increase the spectrum of activity depending on the type of infection a patient is afflicted by. The focus of this section will be on the acquired forms of antibacterial resistance commonly associated with antibiotic use, those acquired via mutations to chromosomal genes or transferred by horizontal gene transfer. These acquired resistance mechanisms generally fall into three categories to be discussed individually; decreasing cellular concentrations of drug through efflux or alterations in membrane penetration, modulation of the target by mutations or post-translational modifications or through the use of inactivating enzymes to neuter a drug's efficacy.

\section{Decreasing cellular concentrations of drug}

Gram-negative bacteria are intrinsically more difficult for a drug to penetrate due to the presence of an outer membrane and the presence of efflux pumps. This outer membrane allows hydrophilic solutes, including antibiotics, to penetrate through porin proteins. These porins are often speculated to be largely indiscriminate in their permeability, size permitting [64]. It has been shown in cases of carbapenem resistance in Enterobacteriacae, Pseudomonoas and Actinetobacter that a reduction in porin expression such as oprD led to drug resistance, and is often the first step prior to the acquisition of extensive carbapenemases $[65,66]$. This response to drug exposure is concerning as it completely precludes a class of drugs based on its physical chemical properties, something that combination therapies cannot overcome. Logically it follows that a downregulation of the bacteria's ability to incorporate soluble nutrients would result in attenuated bacteria, a resultant fitness cost of resistance. However gram-negative bacteria have multiple types of porins that favor different sized molecules and are expressed in response to certain stress elements [67]. For example larger, more indiscriminant porins such as OmpF or those that are solute-selective like LamB glycoporins are expressed in response to nutritional stress. In the cases of antibiotic stress smaller channels such as OmpC are in more abundance, resulting in decreased antibiotic perfusion but also fewer nutrients. Such a balance can theoretically be exploited in combinations and rational drug design and has been a driving force for the development of nutrient state dependent antibiotics such as siderophore conjugates that may be more efficacious if bacteria are in a weakened state [68]. The converse of exclusion-based antibiotic resistance is the bacteria's ability to promote xenobiotic efflux, and is often found to be complimentary to 
changes in porin expression levels [69]. Efflux pumps are often major contributors to intrinsic gram-negative resistance to gram-positive agents and can similarly be overexpressed as a result of antibiotic stress. There are pumps that have very specific substrates such as the Tet pumps for tetracyclines and other, more broadly acting multidrug resistant (MDR) pumps which greatly contribute to drug resistant infections [70]. Genes that encode MDR efflux pumps are found in multiple places on the bacteria genome, and have been shown to mobilize onto plasmids that can be transferred between bacteria, a phenomenon known as horizontal gene transfer. The resistance nodulation division (RND) of MDR pumps is a family found in gram-negative bacteria and is the most studied family of clinically relevant efflux pumps. In E. coli the RND AcrB is a homotrimer inside the inner membrane that is coupled to an outer membrane channel called TolC that allows for complete efflux. The AcrB has two distinct binding pockets that have vastly different properties, which is what allows the efflux pump to have such a wide variety of substrates and cause resistance across many classes of antibiotics [71]. Patients have been identified to have been infected by drug resistant bacteria overexpressing efflux pumps for over 20 years $[72,73]$. Research in drug discovery has put a heavy emphasis on either inhibiting known efflux mechanisms [74, 75] or using medicinal chemistry to avoid efflux issues that prevent efficacy $[39,40]$. Expression of efflux pumps can be tied into alterations to regulatory networks via mutations at local repressors, global transcription factors or inter-gentic sites [76]. Understanding how small molecule intervention as well as nutrient and environmental status affects efflux regulation is another important aspect to consider when developing drugs with resistance in mind.

\section{Target modulation}

In antibiotic drug discovery, we seek to deliver targeted agents to kill bacteria selectively with as few off target effects as possible. As such, most agents have a very specific target that they bind to in order to illicit their effects. For the bacteria this provides an opportunity to develop resistance at the site of action to disfavor antibiotic activity. This can be in the actual binding site, as is the case with sulfonamide resistance [7], or in bioactivation enzymes for prodrug activation as seen in INH with EthA mutations [77]. In a typical infection there are a host of different species of bacteria and subpopulations of different strains. A single point mutation in a gene conferring resistance to an antibiotic gives that strain a much higher survivability chance in cases of chemotherapeutic intervention. The resistant strain is thus favored to proliferate and a high pressure is applied to retain that resistant gene. This is highlighted in linezolid resistance in gram-positive organisms such as $S$. pneumonia and $S$. aureus. Linezolid typically targets the $23 \mathrm{~S}$ rRNA ribosomal subunit, but in resistant mutants there is a T460C mutation in the $r p l C$ gene, something these organisms have multiple copies of. This selected resistant trait quickly undergoes recombination between these homologous alleles which pushes the overall bacterial population to almost exclusively express this resistance mechanism $[78,79]$. Bacteria can also protect an active site without going through genetic mutations. This can be accomplished through post translation modifications of the site, such as methylation via methyltransferases or direct methylases. The erythromycin ribosome methylases $(\mathrm{erm})$ methylate the $16 \mathrm{~S}$ rRNA and disfavor the binding of macrolides, lincosamides and streptogramins [80]. In resistant bacteria the 
$\operatorname{arm} A$ and $r m t$ methyltransferases have been clinically associated with inactivation of aminoglycoside antibiotics, although this is not typically the predominant form of resistance [81]. Plasmid-mediated alterations have been noted in fluoroquinolone resistance, with a repeat in the genes coding for pentapeptide repeat proteins (PRPs) that can restore topoisomerase activity by releasing the quinolone [82]. Some species of bacteria gain resistance to colistin by modulation of regulators that control their lipopolysaccharide (LPS) production, which in turn alters the negativity of the LPS and the binding preference for colistin. The multitude of ways that bacteria can adapt in the face of pressures confers quite the challenge in designing new agents as well as novel drug regimens to stay one step ahead of emerging resistance.

\section{Inactivating enzymes}

Perhaps the bacteria's best line of defense is the internal ability to inactivate a parent compound completely. First discovered to be an issue within months of the first administration of penicillin in the 1940 [83], thousands of different enzymes have been identified that inactivate various antibiotic classes The best example of bacterial inactivation is the $\beta$-lactamase family of enzymes produced in response to the most prevalent class of antibiotics. This series of enzymes is responsible for the hydrolysis of the $\beta$-lactam ring of penicillins, cephalosporins, clavams, carbapenems and monbactams, rendering them incapable of their covalent modification of the penicillin binding protein. Typically these enzymes can be classified structurally into 4 groups conserved amino acid differences (A, B, C, D) or function (serine catalyzed or metalloenzyme) [84]. Serine mediated hydrolysis is accomplished by a covalent nucleophilic attack on the lactam ring system followed by hydrolysis by water to a carboxylic acid. Metalloenzymes use a zinc atom to activate a water molecule to perform a similar hydrolysis, and then stabilize the resulting acid to drive the equilibrium. Especially difficult to treat resistance is associated with extended-spectrum $\beta$-lactamases (ESBLs) and carbapenemases that inactivate the later stage $\beta$-lactams that are typically last line of defense agents in gram-negative infections such as carbapenem resistant Enterobacteriacae (CRE). The most widespread carbapenemase is the New Dehli metallo- $\beta$-lactamase (NDM) that is found in $A$. baumannii, $K$. pneumonia and $E$. coli that confers resistance to all $\beta$-lactams except actreonam. NDM is able to confer amongst many species and spread across the world rapidly due to the high motility of the gene bla $a_{\text {NDM }}$ between plasmid and chromosome [85]. Another example of enzymatic inactivation of an antibiotic utilized by bacteria is the macrolide esterases. Macrolide antibiotics typically have labile ester moieties within their macrocyclic lactone ring that is integral for antibiotic activity. EreA and ereB are two isolated proteins that perform the cleavage of this bond in highly macrolide resistant bacteria [86]. While not a particularly common resistance mechanism, it results in highly resistant erythromycin resistant E. coli and Providencia stuartii and has been identified in $S$. aureus and Pseudomonas. Bacteria are also capable of inactivating antibiotics by the covalent transfer of functional groups to the chemical scaffold to impair target binding. Natural products have a rich history in antibiotic drug discovery, and these larger molecules tend to be quite rich in free hydroxyl and amide groups that are ripe for modifications. Some of these strategies include O-phosphorylation, O-nucleotidylation, O-ribosylation, O-glycosylation, thiol transfers and acylations. With such a wide variety of diversity in their arsenal for this inactivation pathway it is unsurprising that this is one 
of the largest families of resistance enzymes. Aminoglycosides are particularly susceptible to these types of modulations, due to their high molecular weight and abundance of functionalizable groups. In the cases of aminoglycoside acetyltransferases, phosphotransferases and nucleotidyltransferases it has been shown that all three mimic the target ribosomal binding cleft in order to trick the molecule into being a substrate [87].

\section{Discussion: Mitigating Resistance Through Drug Discovery}

To exacerbate the problems of emerging drug resistance all over the world, there has been a dearth of innovation in the form of new antibiotics. Particularly worrisome is the lack of novel chemical classes in this field, the agents that have been developed have primarily been analogs that improve on current agents. This usually results in improved PK properties for more administration options or improves spectrum of activity, but commonly are susceptible to previously emerged resistance mechanisms. Our reliance on antibiotic use is not going to decrease anytime soon; an aging population will require more surgeries, still will undergo immune-suppressing chemotherapeutics and will in general live longer and thus be exposed to more pathogens. Physicians are quickly realizing the benefits of proper antibiotic practices that should slow the emergence of a resistance epidemic, but the need for new agents with the ability to escape known mechanisms that render our front-line therapies ineffective is evident. In order to accomplish this, the biology of resistance obviously needs to be well understood but we also need agents that hit new targets or have novel ways of inhibiting validated targets. Some alternative strategies are being implemented in lieu of novel antimicrobial compounds in the hopes that less stress on the organism will result in less selective pressure to develop resistance. Agents that target virulence factors, toxin secretion, biofilms, cell adhesion components, signaling pathways and modulation of the host immune system and microbiome are all up and coming therapies in this respect [88].

From an antimicrobial perspective, novel mechanisms of action have been scarce since the introduction of the oxalindiones in 2000. Since then, the only first in class compounds approved in the US have been daptomycin, retapamulin, fidaxomicin and bedaquiline [89]. These agents have very narrow spectrum of activity, none of which are efficacious against gram-negative bacteria. It should be noted that a new $\beta$-lactamase inhibitor has been approved that greatly aids the Fight in multi-drug resistant gramnegative infections. Avibactam is a non- $\beta$-lactam that has demonstrated inactivation of several important $\beta$-lactamases including Klebsiella pneumonia cabapenemase (KPC) and AmpC-type cephalosporin inactivators. There must be a paradigm shift in the drug discovery field; the amount of drug resistant gram-negative infections continues to climb with no relief beyond newer $\beta$-lactam agents [90]. Even the recently discovered and highly heralded teixobactin is limited to gram-positive infections and is far away from being clinically relevant, although its isolation gives great hope in the discovery of a natural product derivative with potent activity [91]. 
Similar to gram-negative pathogens, tropical disease areas such as tuberculosis have gained relatively little in the ways of novel chemistry added to their arsenal and have a disturbing uprising in resistance cases. Tuberculosis primarily afflicts poorer regions of the world and as a result has lacked funding and effort in new drug discovery. Resistance threatens an effective drug regimen to treat the disease, but it also forces patients to extend an already lengthy therapy time as well as utilize more toxic agents that may be difficult to obtain. With only two limited approvals since the discovery of rifamycin in the 1960's, the field is desperate for new agents. A struggling drug pipeline is not very encouraging and there are many challenges associated with targeting the mycobacterium as well as circumventing its known resistance pathway. In the next chapter we describe efforts to develop new inhibitors against two drug targets that act on cell wall construction, perhaps Mtb's strongest natural defense. First we are exploring an extremely hot field of anti-tubercular drug discovery by targeting a membrane transport protein responsible for shuttling a critical membrane component to the outer portions of the cell. Next we re-investigate the Fatty Acid Synthesis (FAS) II pathway, targeted by multiple first and second choice drugs used for TB. In doing so, we are taking a unique approach that is gaining popularity in other fields of drug discovery and should circumvent both resistance and toxicities associated with previous inhibitors. Both projects highlight the underlying need for innovation; whether it is inhibiting a new target or finding a unique way to hit a validated target, both are cognizant of emerging resistance and take aim at generating useful chemistry for the future.

The history of antibiotics has given us a large knowledge base of pharmacology and biology of existing therapeutics. We know there are biosynthetic pathways that are critical for bacterial growth and survival, and we have agents that target many steps in these cascades. The example often used in this regard is the folate biosynthesis pathway. Sulfonamide antibiotics are the oldest synthetically derived class that are still highly clinically relevant today. These work on the heavily studied DHPS enzyme, and are often used in conjunction with a DHFR inhibitor such as TMP to treat a wide variety of pathogens. Using the knowledge the field has acquired on this pathway, it only makes sense to find new ways of both targeting and exploring clinical usefulness of inhibition of folate production. In chapter three we describe two projects on doing both. First the development of inhibitors of another essential enzyme in this pathway, using structure guided and fragment based drug design. Developing a new inhibitor of another enzyme will circumvent resistance to sulfonamides and could be just as synergistic with TMP for robust clinical efficacy. The next project will be a drug repurposing screen to utilize both known bioactive molecules as well as potent inhibitors of DHPS to try and find new agents to treat Pneumocystis pneumonia (PCP). Efforts such as these are gaining popularity in the drug discovery field, especially in antibiotics because of the cost effectiveness and relatively high success rate. In terms of the bioactive agents, the data in terms of safety and therapeutic index is already acquired, so rapid decision making can occur to prioritize lead molecules. Exploring DHPS inhibitors is promising for this disease indication as SMX is already the drug of choice in curative and prophylactic treatment of this opportunistic organism. As these studies have gone on, we have generated more hypotheses that can further answer some emerging debate on DHPS inhibition in general as well as its role in PCP infection treatment. 


\section{CHAPTER 2. TARGETING THE CELL WALL OF MYCOBACTERIUM TUBERCULOSIS}

\section{Introduction: TB as a Global Threat}

Mycobacterium tuberculosis (Mtb) has been a major human pathogen since the dawn of modern human existence, with the evolution of modern Mtb sublineages closely correlating with the different waves of human migration out of Africa [92]. As the human race began to form denser population centers, culminating in urbanization, Mtb spread more easily, and it became one of the leading causes of death by the end of the twentieth century. The prognosis for patients with tuberculosis (TB) improved dramatically with the discovery and introduction of antitubercular drugs, starting with streptomycin in 1946 and subsequent research that produced today's front-line therapies for drug-sensitive Mtb. It is important to note that despite having an effective treatment regimen, Mtb still caused an estimated 9 million infections and 1.5 million deaths in 2013 [93].

The current standard of care for drug-susceptible Mtb infection is very effective in bacillary clearance, provided full compliance by the patient. A 2-month "intensive" phase of a four-drug cocktail containing rifampicin (RIF), isoniazid (INH), pyrazinamide (PZA) and ethambutol (EMB) (Figure 2-1) is followed by a longer "continuation" phase of RIF and INH to eradicate the remaining bacilli that have entered a dormant, non-replicating latent phase. A looming global issue hidden in the midst of this epidemic was the emergence of drug-resistant bacteria, a trend that is on the rise, as such strains are easily spread with low fitness costs associated with transmission $[61,94]$. The World Health Organization (WHO) reported that globally $3.5 \%$ of naïve infections already expressed resistance to two most efficacious front-line agents used to treat the disease, RIF and $\mathrm{INH}$, thereby classifying the infection as multidrug-resistant tuberculosis (MDR-TB) [93]. Treatment of drug-susceptible Mtb is difficult already, requiring 6 to 9 months of combination therapy in ideal circumstances. Complicating the issue is that Mtb tends to be endemic to the developing world; thus, access to adequate healthcare facilities and drugs can be limited for those patients. This leads to incomplete treatments, a leading driver for conferring drug resistance; $20.5 \%$ of patients with relapsed disease have MDRTB [93].

Treatment for MDR-TB can extend upwards of 2 years and relies on more toxic, less efficacious second- or third-line agents, many of which are even more scarce than front-line drugs in affected areas [95]. A very common and deadly complication of Mtb infection is coinfection with human immunodeficiency virus (HIV) $[44,96]$. This is particularly troublesome because RIF, a mainstay in Mtb therapy, is a potent inducer of drug-metabolizing enzymes, including cytochrome P450 (CYP) 3A4. This induction dramatically reduces plasma levels of several highly active antiretroviral therapy drugs; thus, patients are often forced to complete their TB treatment before beginning HIV treatment $[43-45,96]$. Patients who contract MDR-TB with HIV have a very poor prognosis due to the duration of treatment; these individuals frequently succumb within a few months. 

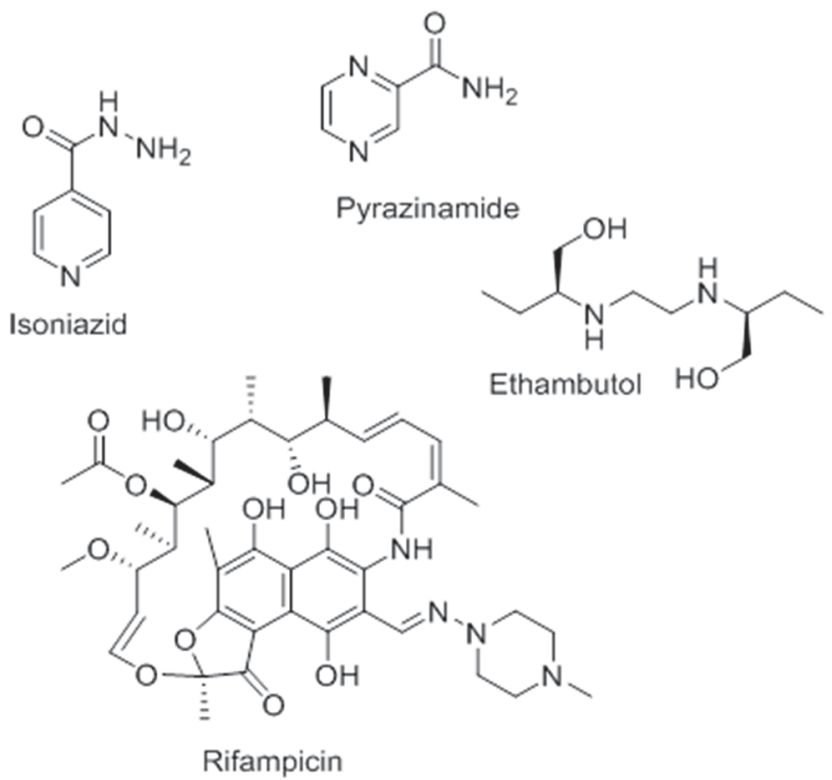

Figure 2-1. Front line therapy of tuberculosis.

Reprinted with permission from Bentham Science. Hoagland, Daniel T et al (2016). "Advances in Drug Discovery and Development for Pediatric Tuberculosis." MiniReviews in Medicinal Chemistry 16(6): 481-497 
The urgent need to develop new active agents to combat MDR-TB has been compounded by the emergence of extensively drug-resistant tuberculosis (XDR-TB) [97], defined as MDR-TB with additional resistance to fluoroquinolones and one of the injectable second-line agents. Furthermore, cases of totally drug-resistant tuberculosis (TDR-TB) have been noted in China, India, Africa, and Eastern Europe. In TDR-TB, the mycobacterium are resistant to all available therapeutics [98]. Combining the decline in efficacy of known active agents with the dearth of new chemical entities being moved into the clinic setting, we have reached the point where a "postantibiotic" era is a very real possibility [99]. From a clinical perspective, the WHO's approach to reduce the number of TB deaths and infections is to implement directly observed treatment, short course. This regimen implements the following five main elements to increase survival and decrease transmittance for most developing countries: (1) government commitment to monitoring and treating Mtb infections; (2) sputum-smear microscopy for all cases to assess drug susceptibility; (3) standardized treatment regimens with direct observation by a trained healthcare professional; (4) uninterrupted drug supply; and (5) standardized reporting system to maximize data interpretation directly from patients' medical records. This approach has reduced the overall number of TB infections and deaths, but it has not affected the increasing numbers of MDR-TB infections [100]. Therefore, more chemotherapeutic interventions are needed. New agents would ideally have the following attributes: a novel mechanism of action to attenuate cross resistance; rapid bactericidal activity to reduce duration of therapy; optimized pharmacokinetic/pharmacodynamic (PK/PD) properties for once daily oral administration; low potential for drug-drug interactions to allow combination therapy, especially with other TB drugs and current HIV therapeutics; and excellent safety profile to allow for use in children and pregnant women. These ideal criteria are coupled with other practical goals such as inexpensive manufacturing, high compound stability, narrow spectrum of activity, high tolerability, and a low rate of spontaneous-resistance emergence.

In recent years, we have seen the first regulatory approvals for new Mtb drugs (i.e., bedaquiline and delamanid) in decades (Figure 2-2) [55, 101]. Both drugs have been conditionally approved [delamanid in the European Union's European Medicines Agency (EMA)[102]] because adverse events have been noted. The U.S. Food and Drug Agency (FDA) has approved bedaquiline for MDR-TB and delamanid as a compassionate care option for XDR-TB and TDR-TB infections, and the EMA approved both agents for MDR-TB. The fact that these drugs have pronounced issues, including being very highly lipophilic, and the lack of viable alternatives in late-stage clinical development is indicative of the state of drug discovery in this field. In this review, we discuss up-and-coming drug classes that are on the cusp of entering clinical trials. We focus on the novelty of their mechanisms of action, the advantages they may possess in targeting MDR-TB in replicating and nonreplicating forms, and their prospects moving into the clinic. 


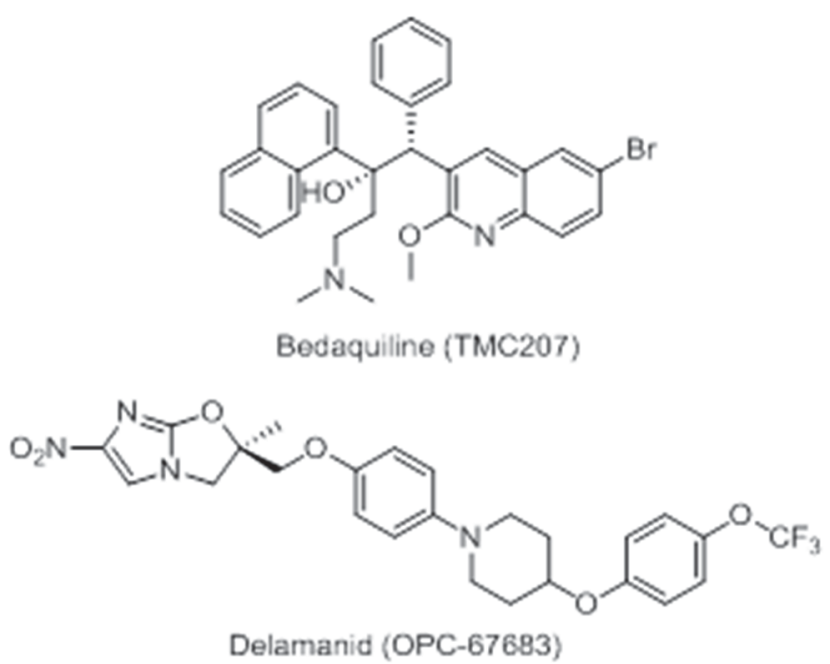

\section{Figure 2-2. Bedaquiline and Delamanid}

Two recently approved drugs for the treatment of MDR-TB, both with issues that limit their usage

Reprinted with permission from Elsevier Science. Hoagland, Daniel T et al (2016). "New Agents for the Treatment of Drug-Resistant Mycobacterium Tuberculosis." Advanced Drug Delivery Reviews 102(1): 55-72 


\section{M.tb Life Cycle and Microenvironments}

M.tb infection begins upon pulmonary exposure, following inhalation of active bacilli from the surrounding environment. The risk of being infected is greatly influenced by many factors, most of which stem from living and working conditions. Population density, weather, duration and intensity of exposure to the M.tb (cramped living and work environments with poor ventilation), a set of virulence factors unique to the infecting organism, and relative immunocompetency of the would-be host are all variables associated with succumbing to an M.tb infection. Initial infection occurs when a carrier exposes M.tb to the air via coughing. Small droplets are expelled from the lungs that contain small number of individual organisms $(<10)$ that must be inhaled deep into the lungs. At this point host macrophages in the lung attempt to phagocytize the pathogen (Figure 2-3A) and transport it across the alveolar epithelium and into the lung. This triggers a proinflammatory response that will recruit other immune cells to form an encapsulated granuloma, which is a typical immune response to a pathogen. At this point, most of the bacterial burden is contained inside what are now considered foamy macrophages (Figure 2-3B), which begin to line the outside of the granuloma. When the granuloma is first formed, it is well vascularized and a lot of immune cells are present (Figure 2-3C), which bolsters the ability of drugs to reach the infection and the host's immune system to combat the pathogen. As the granuloma continues to mature against the relentless immune response, the outer wall begins to harden with a thick fibrous capsule, and the inner core is walled-off from the immune cells. Foamy macrophages line the outside of this lesion, cells die and caseum develops at the core, which cuts off the remaining vasculature. At this point, the granuloma is considered necrotic, and the bacilli exist extracellularly in this caseum and can enter their largely dormant state (Figure 2-3D). Necrotic granuloma lesions (also known as tubercles) make drug penetration difficult and render mouse infection models that do not recapitulate this complex pathology poor surrogates for the human disease. After years or in cases of immune system compromise, the granulomas will fuse to the airways of the lungs and burst, releasing the pathogen to spread to new tissue and new hosts (Figure 2-3E) [103]. Each of these points in the Mtb life cycle have different microenvironments that can affect drug susceptibility. For example, various degrees of vasculature result in lower blood flow, decreased oxygen levels, and $\mathrm{pH}$ differences in the intra/extracellular fluids that can affect ionization or activation of drugs, drug targets in Mtb may be turned off, depending on the replication stage (latent vs. actively replicating), and the ability of the drug to penetrate tissues and membranes required to reach the mycobacteria.

\section{TB in Children}

Tuberculosis in pediatric populations is a horrible and often under reported affliction that suffers from problematic diagnosis and child specific treatment options. Children are often a lower priority than adults in many healthcare systems due to perceptions of a lack of infectiousness, difficulty in obtaining diagnosis using sputum samples and their rapid progression to a more serious disease state [104, 105]. Globally children under the age of 15 accounted for half a million infections in 2011 with 64,000 


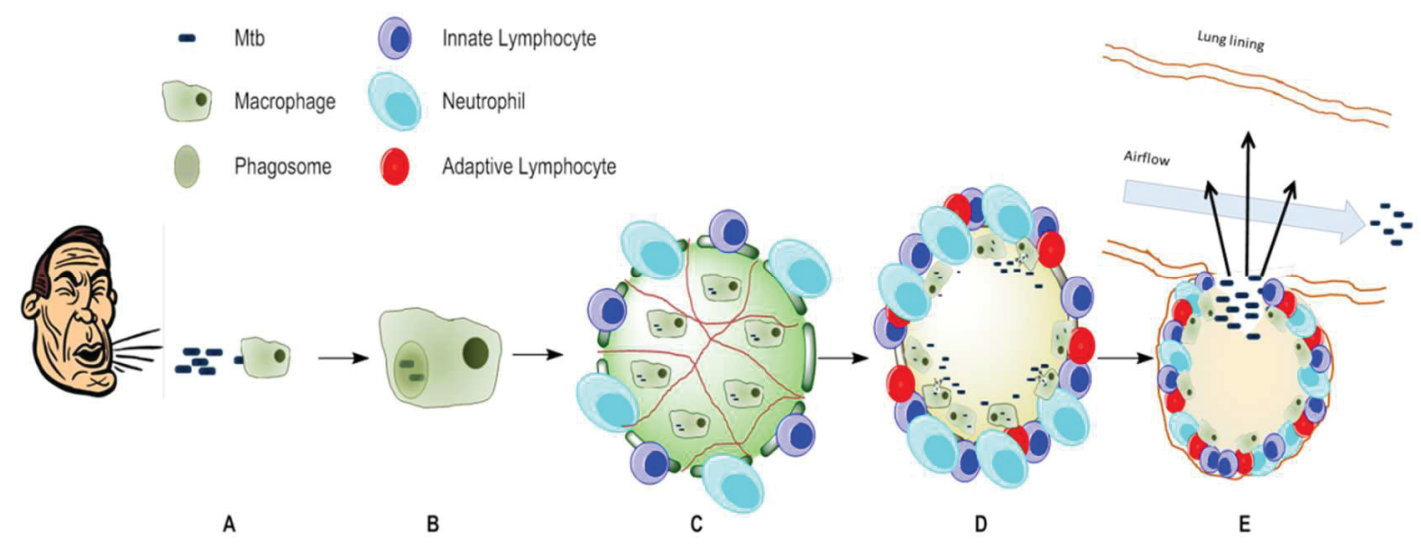

Figure 2-3. A simplified depiction of the Mtb lifecycle

A.) Initial transmission via aerosolized active mycobacterium. Initial bacterial load met by macrophages and phagocytosis occurs to quell the infection.

B.) Mtb inside the phagosome of the host macrophage. Nutrient deplete environment where Mtb relies on host carbon sources like cholesterol degradation and the MCC

C.) The initial immune response to wall off infected macrophages inside a granuloma using inflammatory response cells. Containment is successful for $90 \%$ of immunocompetent patients. Immuno-suppression can lead to rapid dissemenation. Granuloma still well vascularized.

D.) Necrotic granuloma where Mtb begins to seep out of the macrophages into the extracellular caseum layer. Vasculature has been choked out by thick caseum layer. This phase can last decades or until host becomes immuno compromised

E.) Eventual granuloma rupture that allows Mtb to escape into the airways and repropagate the cycle and infect others.

Reprinted with permission from Elsevier Science. Hoagland, Daniel T et al (2016). "New Agents for the Treatment of Drug-Resistant Mycobacterium Tuberculosis." Advanced Drug Delivery Reviews 102(1): 55-72 
deaths in HIV negative patients. Children under the age of five are several times more likely than adults to progress to more serious extra pulmonary dissemination involved in miliary TB and TB meningitis [104, 106-108] and most will succumb to infection within a year of initial exposure [109]. With this rapid progression from infection to disease state, children can be quite indicative of an active epidemic at a local level and are useful in distinguishing between latent and infectious populations. Neonates have the highest progression rate to the disease state and in fact can be useful in diagnosis of previously unknown TB in the mother [110]. As a much understudied population, there is little clinical or even pre-clinical data behind the dosing regimens for pediatrics. There is a significant difference in drug delivery, both in their pharmacokinetics and pharmacodynamics [111-115] when considering a child taking the same medication as an adult. Children tend to have a much more sensitive therapeutic index [116-118], with noted adverse effects to TB therapies worse than adults, especially for second-line agents used for MDR-TB. We will discuss this more in depth later in this review, but for now it should be noted that there is a dearth of knowledge for this patient subpopulation that is just now starting to be addressed. Before 2012, the WHO did not distinguish between pediatric and adult infections when issuing their annual estimate of epidemiology. In addition, the DOTS strategy largely neglects children, as the primary means of detection is sputum smear-positive, which pediatrics often don't display [104, 106, 119]. Other factors contribute to the delicate disease state of pediatric TB infections including altered immune response [120,121], difficulties in proper dosing (both amounts and formulation)[122], and the rapid progression to severe disease state. We will discuss the future of small molecule M.tb therapeutics, specifically how it pertains to the pediatric population, as well as alterations in screening and target validation strategies that are proving helpful in the identification of lead candidates to be advanced to the clinic.

\section{Agents to Treat TB and Known Issues in Adults and Children}

The normal TB drug therapy is 6-9 months of the previously discussed front-line agents. Once a patient no longer responds to INH and RIF they are classified as having MDR-TB, and further resistance to a fluorquinolone or injectable aminoglycosides they are deemed to have XDR-TB. These resistant infections are much more difficult to treat and require longer therapy duration with more toxic or less efficacious drugs. Agents typically reserved for MDR or XDR infections are grouped into four classes (in addition to the first line class) based on efficacy, potency, drug class and experience of use [95, 116] (Figure 2-4). The first group of second-line agents includes injectable aminoglycosides and polypeptides. The next group is the fluoroquinolones, in both oral and injectable forms, from which generally only one agent is used. The final group of second-line agents are also oral and of various drug classes. A fifth group exists as a third-line of anti-TB drugs which include several repurposed compounds such as clofazimine (leprosy), Linezolid (respiratory infections), amoxicillin plus clavulanate (broad spectrum use), imipenem plus cilastatin (broad spectrum), and clarithromycin(respiratory infections). Figure 2-4 depicts a common progression method to select treatment combinations between these groups of second-line agents as resistance 


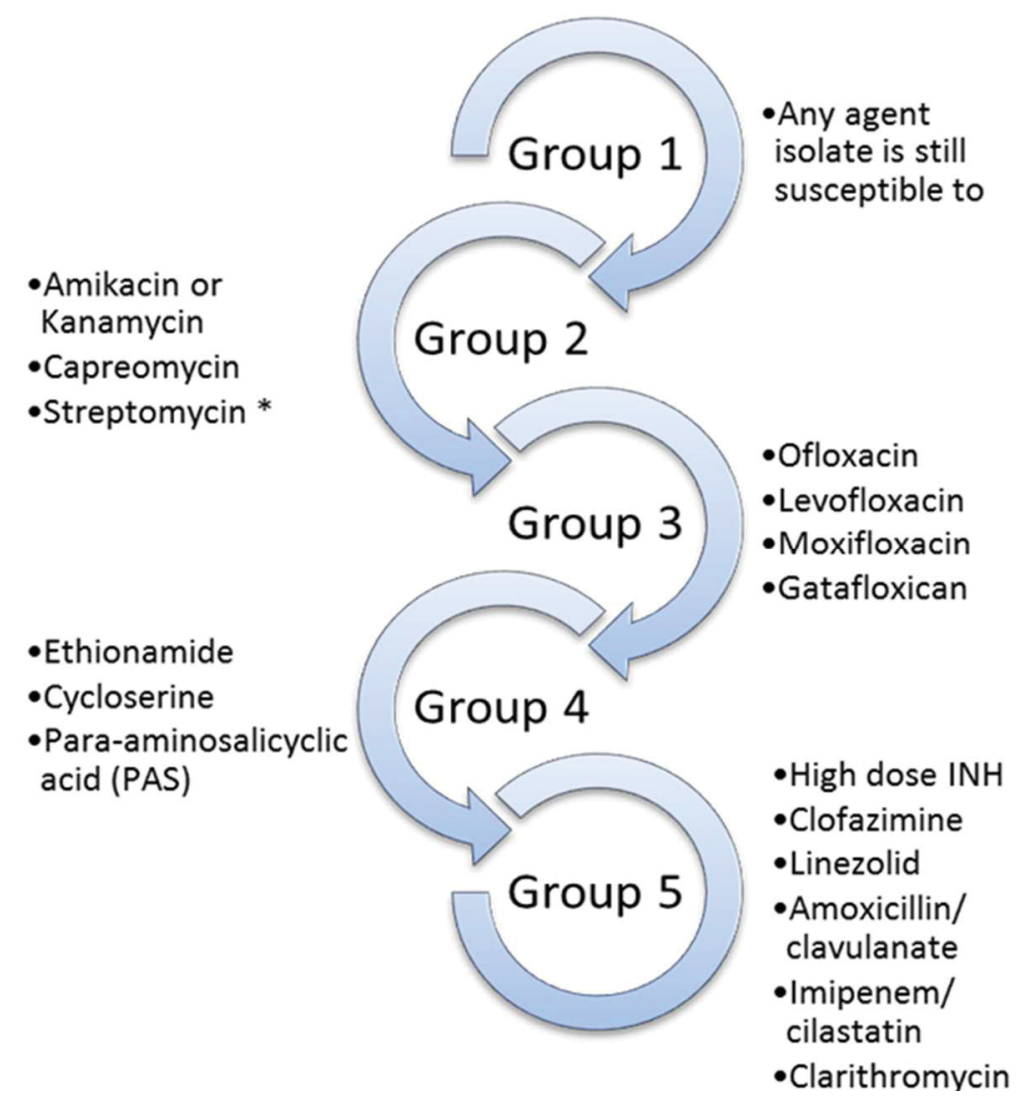

Figure 2-4. General progression of agent addition to MDR-TB infection regimen. Always add one agent until four active agents are being used. *Streptomycin used only if resistance to Amikacin, Kanamycin, and Capreomycin is noted

Reprinted with permission from Bentham Science. Hoagland, Daniel T et al (2016). "Advances in Drug Discovery and Development for Pediatric Tuberculosis." MiniReviews in Medicinal Chemistry 16(6): 481-497 
is confirmed. Typically physicians will attempt to always be using at least four drugs that the isolate is susceptible to in order to prevent further resistance from emerging.

\section{Adverse Effects of Front-line Agents}

As previously stated, TB primarily affects impoverished portions of the world and therefore the majority of people being treated for TB live in very resource-scarce areas. This not only makes obtaining the necessary agents required for first or second-line therapy difficult; it also blurs the line for monitoring adverse effects from therapy. Reporting an adverse reaction is very tricky, as many in this patient population are extremely malnourished and co-infected with one or more serious diseases. However, as of late, there has been a more concerted effort in keeping track of toxicological events as it pertains to tuberculosis treatment, as doses must be safe and well tolerated in order to maintain a high level of patient compliance [123-125]. This is something as a field that needs to be considered going forward as new agents and regimens are developed.

We will first start with side effects associated with front-line therapies. Two months of RIF (10-20 mg/kg, max dose $600 \mathrm{mg} /$ day), PZA (30-40 mg/kg), INH (10-15 $\mathrm{mg} / \mathrm{kg}$, max dose $300 \mathrm{mg} /$ day) and sometimes EMB $(15-25 \mathrm{mg} / \mathrm{kg}$, in areas of high HIV or INH resistance prevalence) followed by four months of RIF and INH at the above doses. Despite the recent increase in dosage, it has been discussed by Hiruy et al. that these doses might be inadequate to reach optimal therapeutic plasma concentrations in children [122], a subject that definitely merits more investigation. We will focus on noted adverse effects for current or lower doses of front and second-line agents.

INH has two major reactions, neurologic and hepatic, both of which are relatively rare. INH competes with vitamin B6's (pyridoxine) action as a cofactor in synaptic neurotransmitter biosynthesis [126]. Dose-dependent neurologic toxicity is less common in children than in adults, which results in peripheral neuropathy, clonic seizures and paresthesia [127]. These effects are magnified in patients co-infected with HIV or who have vitamin deficiencies. Pyridoxine supplementation is recommended in patients whom are severely malnourished or co-infected with HIV [118]. Dosage of INH is dependent on a specific genotype ( $\mathrm{N}$-acetyltransferase 2 ) of a patient population, as it pertains to their rate of acetylation [111]. Slow acetylators are associated with more toxicity issues [124, 128] than intermediate or fast acetylators and genotype differs amongst ethnic groups. Hepatitis is the most severe reaction to $\mathrm{INH}$, with rare reports occurring in children receiving low doses $[129,130]$. However, INH is commonly given prophylactically to help clear latent infections. Systemic hepatotoxicity is rare in children and shown in rather large patient studies to have no effect on discontinuation of therapy [131]. Cases that do devolve into liver dysfunction can result in organ failure and require transplantation $[132,133]$.

RIF is notorious for wreaking havoc on other drugs co-administered with it if they are subject to hepatic metabolism. RIF is a potent ligand for the nuclear hormone receptor PXR, an inducer of Cytochrome p450s (notably 2C9 and 3A4), P-glycoprotein (Pgp or 
MDR1), ATP binding cassette transporters, lung resistance-related proteins and phase II conjugation enzymes [44, 46, 134-137]. The most troublesome effect is the induction of CYP3A4, which is responsible for the metabolism of many anti-retroviral protease inhibitors, making co-administration of TB and HIV drugs problematic in many parts of the world where therapeutic options for both conditions are limited. There is very limited evidence of RIF having adverse hepatic effects when administered alone in children. A common, albeit benign, side effect of RIF is the discoloration of bodily fluid to an orange-red color that may impart some social stigma. Other rifamycins are being developed with similar potency as RIF but with fewer pharmacokinetic disturbances and greater plasma exposure which will be discussed later.

An integral part of the intensive treatment phase of pediatric and adult drug susceptible TB infections is PZA. PZA is a unique drug that barely exhibits any antitubercular activity in vitro. However, its introduction to front-line therapy reduced therapy times by three months. PZA requires acidic conditions such as inside a macrophage and in granulomas to obtain optimum activity, the exact mechanism of action of pyrazinamide remains a subject of conjecture [138], but it has been hypothesized that bioactivation by pyrazinamidase to pyrazinoic acid within the M.tb cell causes disruption in membrane potential and energy production [139]. The commonly associated complications with PZA therapy are GI distress, hepatotoxicity and joint discomfort. In adults, hepatotoxicity is dose and duration of therapy dependent [124]. Hepatotoxicity from PZA has been shown to be relatively rare in children with little alteration in hepatic enzyme function $[113,140]$. Joint problems stem from non-gouty polyarthralgia and gout-induced arthritis via decreasing renal excretion of uric acid. It has not been studied in children, but in adults there have been slight increases of systemic uric acid levels leading to arthritis symptoms.

EMB was developed as a replacement for Thioacetoazone (TAC) in front-line therapy and serves a very similar role in the combination. EMB is only moderately active against M.tb but plays an important role in suppression of resistance emergence in the drug cocktail. TAC is a thiosemicarbazone that can cause extreme skin reactions, such as lethal Stevens-Johnson reactions, in HIV co-infected patients. Its use is now limited to the most drug-resistant MDR/XDR infections. EMB is not without toxicity concerns, being contraindicated in use for pediatrics up until several reviews of pharmacokinetics, efficacy and toxicity by the WHO and others [141-143]. The biggest concern with EMB use is the development of optic neuritis, an inflammation of the optic nerve [144]. In the case of EMB-induced neuritis, the inflammation occurs at the retrobulbar (posterior) of the nerve and thus is difficult to detect via normal opthalmoloscopic examination. Disease-state diagnosis is very dependent on patient observation and communication of a loss of visual acuity and color vision for which there is difficulty in tracking such an adverse effect in young children (under the age of five) due to their inability to either comprehend changes in their vision or articulate them to a healthcare provider [142, 144]. Retrobulbar neuritis is reversible, if detected early, and therefore early signs of the disease-state progression are imperative. Recent advances in detection could allow for rapid detection of nerve damage and thus discontinuation of treatment. Specifically the recording visual-evoked potentials (VEPs) in children under the age of five has been 
tested to see if early detection is possible without being reliant on patient communication of distress [145]. Ocular toxicity with EMB is dose and duration of therapy dependent, with $40 \%$ of adults developing symptoms at doses greater than $50 \mathrm{mg} / \mathrm{kg}$ and $0-3 \%$ at 15 $\mathrm{mg} / \mathrm{kg}[144]$.

\section{Adverse Events to Second-Line Therapies}

Second-line therapeutics for treating drug-resistant TB have their own unique, and often worse, adverse events especially in children with toxicity occurring in up to $40 \%$ of cases [146]. Any treatment for multi or extensively drug-resistant pathogens will always have a slightly larger toleration to adverse events just because the disease state is much more life threatening. The use of one injectable antibiotic is standard in second-line therapy for drug-resistant infections. Second-line therapy is now often supplemented with broad spectrum antibiotics such as fluoroquinolones and linezolid which have a better efficacy and safety profile than historic second-line agents such as ethionamide [147-153].

Important recent studies have begun to focus on developing optimal regimens for the pediatric MDR-TB population. These studies include an examination of pharmacokinetics specific to pediatrics. There are studies of aminoglycosides given short course through IV, but little data on the kinetic profile of those dosed long-term, as in the case with TB therapy, which is generally given intramuscularly (IM) [154, 155].

Injectable agents commonly include aminoglycosides such as amikacin and kanamycin and the cyclic polypeptide capreomycin. Typically the aminoglycosides are given first with favor going towards Amikacin, due to its more potent MIC, with capreomycin withheld for XDR infections $[116,156]$. With a high degree of cross-resistance noted between the aminoglycosides, if resistance develops to one agent, it is generally recommended to progress to capreomycin or another class. The injectable antibiotics are associated with extensive ototoxicity. As discussed before, when dealing with pediatrics, especially infants and very young children, diagnosis of these adverse effects are quite difficult. The next class for MDR infections are the fluoroquinolones, most common being moxifloxacin and levofloxacin. Fluoroquinolones have excellent orally bioavailability and can be administered PO daily, are highly active and display little cross resistance to other TB therapeutics [157]. The fluoroquinolone class of antibiotics has well documented toxicity issues from their wide ranging use, most notably peripheral neuropathy, weakening and inflammation of tendons, dysglycemia and prolongation of QT intervals with associated cardiotoxicity. Other agents used for the treatment of MDR and XDR-TB are listed with their noted adverse events in Table 2-1. The need to develop new therapeutics to treat drug-resistant TB without these negative events is imperative if we want to not only cure patients afflicted by MDR-TB, but also increase their subsequent quality of life after beating their infection. 
Table 2-1. Groups of commonly administered agents for MDR and XDR infections and their noted adverse events in children

\begin{tabular}{|c|c|c|c|}
\hline Group & Group Eame & Drugs & Adverse Effects \\
\hline 2 & Injectable Agents & $\begin{array}{l}\text { Amikacin, Kanamycin, } \\
\text { Capreomycin, } \\
\text { Streptomycin }\end{array}$ & $\begin{array}{l}\text { Vestibular toxicity, } \\
\text { nephrotoxicity, auditory } \\
\text { toxicity }\end{array}$ \\
\hline 3 & Fluoroquinolones & $\begin{array}{l}\text { Moxifloxacin, } \\
\text { Levofloxacin, Ofloxacin }\end{array}$ & $\begin{array}{l}\text { Sleep disturbance, GI } \\
\text { distress, arthritis, peripheral } \\
\text { neuropathy, prolonged QT }\end{array}$ \\
\hline \multirow[t]{3}{*}{4} & $\begin{array}{l}\text { Bacteriostatic } \\
\text { Second-line (Oral) }\end{array}$ & Ethionamide & $\begin{array}{l}\text { GI distress, hepatotoxicity, } \\
\text { hypersensitivity, metallic } \\
\text { taste }\end{array}$ \\
\hline & & Cycloserine & $\begin{array}{l}\text { Neurological distress, } \\
\text { psychosis, convulsions, rash, } \\
\text { drug interactions }\end{array}$ \\
\hline & & $\begin{array}{l}\text { Para-aminosalicyclic } \\
\text { acid }\end{array}$ & $\begin{array}{l}\text { GI distress, hypersensitivity, } \\
\text { hepatotoxicity, sodium load }\end{array}$ \\
\hline \multirow[t]{6}{*}{5} & $\begin{array}{l}\text { Unclear M.o.A or } \\
\text { significant adverse } \\
\text { events }\end{array}$ & Clofazimine & $\begin{array}{l}\text { GI distress, skin } \\
\text { discoloration, organ damage } \\
\text { due to crystal deposition }\end{array}$ \\
\hline & & Linezolid & $\begin{array}{l}\text { GI distress, headache, } \\
\text { myelosuppression, } \\
\text { neurotoxicity, lactic acidosis, } \\
\text { pancreatitis }\end{array}$ \\
\hline & & High dose INH & $\begin{array}{l}\text { Hepatitis, peripheral } \\
\text { neuropathy }\end{array}$ \\
\hline & & $\begin{array}{l}\text { B-lactam/ enzyme } \\
\text { inhibitor } \\
\text { •Amoxicillin } \\
\text { • Meropenem } \\
\text { •Imipenem }\end{array}$ & $\begin{array}{l}\text { GI intolerance, } \\
\text { hypersensitivy, seizures, } \\
\text { liver, renal dysfunction }\end{array}$ \\
\hline & & Thiacetazone & $\begin{array}{l}\text { Stevens Johnson Syndrome } \\
\text { w/HIV co-infection, GI } \\
\text { intolerance, hepatitis }\end{array}$ \\
\hline & & Clarithromycin & $\begin{array}{l}\text { GI intolerance, rash, } \\
\text { hepatitis, prolonged QT and } \\
\text { ventricular arrhythmias }\end{array}$ \\
\hline
\end{tabular}




\section{Drug Resistance in Mtb}

Drug resistance in M.tb was noted soon after the introduction of streptomycin, the first drug to treat tuberculosis. Exposure to a drug induces stress responses inside the infected cell that favors both genetic and physiological mechanisms that lead to colony survival. Current antitubercular drugs are commonly associated with specific resistance mutations (Table 2-2). Most M.tb resistance observed in the clinic can be attributed to independent, spontaneous mutations that interfere with the drug binding to the target protein, reduce prodrug-activating enzymes, or overexpress an essential target [158]. For a more detailed analysis of the molecular basis of resistance to currently used agents, we refer readers to a review by Palomino et al. [27]. In recent years, our ability to study resistance mechanisms has been greatly aided by the availability of whole-genome sequencing. This cutting-edge technology has not only shed light on many direct and indirect routes of resistance but also added insight into the function of some altered genes, thereby providing crucial information about new drug targets and rationale for future drug-design studies. For example, extensive knowledge has been gained in the areas of lipid metabolism, cell wall homeostasis, purine metabolism, and transcriptional regulation [159]. However, many resistant strains that have been sequenced express additional or unique mutations that differ from commonly associated alterations. Resistance mutations often have an associated fitness cost [160], i.e., the physiological penalty for developing said drug resistance is that the M.tb's growth may be limited in vivo. Bacteria can overcome this fitness via compensatory mutations that allow the organism to act more energetically (similar to the wild-type bacteria), while maintaining resistance. An example from MDR-TB clinical isolates in Russia identified rpoA and rpoC as compensatory mutations in RIF-resistant strains [161]. These genes are mutated to alleviate the fitness costs associated with the drug-resistant rpoB mutation that modulates the binding site at the $\beta$-subunit of the RNA polymerase. Medicinal chemistry strategies to target drug-resistant TB infections include designing agents that have novel mechanisms of action and are thus not prone to cross-resistance or redesign of inhibitors to target the mutant enzyme form, and identification and development of agents for which the fitness cost of further resistance development is high.

\section{Notable Recent Advances in Drug Discovery Technologies for Mtb}

As discovery and development of novel chemical entities to treat Mtb has increased significantly in recent years [150, 162-164] so have the technologies used to discover new agents. New technologies have been developed to complement our increased understanding in the biology of the Mtb life cycle and the success and failures of prior drug development strategies. While there is not space to adequately discuss all these advances in this review we do briefly want to discuss the impact of three key technologies - genomics, screening under defined growth conditions that recapitulate various microenvironments of the Mtb life cycle, and Multicenter matrix-assisted laser desorption/ionization mass spectrometry (MALDI-MS) imaging of drug distribution within the infected lung. 
Table 2-2. Commonly associated drug resistance mechanisms to clinically relevant drugs to treat Mtb

\begin{tabular}{|c|c|c|}
\hline Drug Name & Mechanism of Action & Mechanism of Resistance \\
\hline Rifampicin & $\begin{array}{l}\text { RNA synthesis } \\
\text { inhibition }\end{array}$ & $\begin{array}{l}\text { Mutation of } r p o B \text { induces a } \\
\text { conformational change at } \beta \text { - } \\
\text { subunit of RNA polymerase } \\
\text { causing a decrease in binding } \\
\text { affinity }\end{array}$ \\
\hline Isoniazid & $\begin{array}{l}\text { Mycolic acid } \\
\text { biosynthesis inhibitor } \\
\text { and effects on DNA, } \\
\text { lipid, carbohydrate, and } \\
\text { NAD metabolism }\end{array}$ & $\begin{array}{l}\text { Kat } G \text { suppression causing } \\
\text { decreased pro-drug activation, and } \\
\text { a mutation in the promoter region } \\
\text { of InhA causing an overexpression } \\
\text { of InhA }\end{array}$ \\
\hline Pyrazinamide & $\begin{array}{l}\text { Not fully resolved, may } \\
\text { include membrane } \\
\text { potential disruption }\end{array}$ & $\begin{array}{l}\text { Mutations in } p n c A \text { reducing } \\
\text { conversion to active acid form }\end{array}$ \\
\hline Ethambutol & $\begin{array}{l}\text { Arabinogalactan } \\
\text { biosynthesis inhibition. }\end{array}$ & $\begin{array}{l}\text { Mutations in } e m b B \text { at codon } \\
\text { embB306 }\end{array}$ \\
\hline \multirow[t]{2}{*}{ Amikacin/Kanamycin } & $\begin{array}{l}\text { Protein synthesis } \\
\text { inhibition }\end{array}$ & $\begin{array}{l}\text { 16S rRNA target site modulation } \\
\text { (1400 and } 1401 \text { rrs gene) }\end{array}$ \\
\hline & & $\begin{array}{l}\text { Increased drug inactivation via } \\
\text { overexpression of eis } \\
\text { aminoglycoside acetyltransferase }\end{array}$ \\
\hline Capreomycin & $\begin{array}{l}\text { Protein synthesis } \\
\text { inhibition }\end{array}$ & $\begin{array}{l}\text { Cross resistance with } \\
\text { aminoglycosides plus mutation of } \\
\text { tlyA which decreases rRNA } \\
\text { methyltransferase activity }\end{array}$ \\
\hline Streptomycin & $\begin{array}{l}\text { Protein synthesis } \\
\text { inhibition }\end{array}$ & $\begin{array}{l}\text { Mutations in } r p s L \text { and } r r s \text { confer } \\
\text { binding site modulation }\end{array}$ \\
\hline Fluoroquinolones & $\begin{array}{l}\text { DNA gyrase and } \\
\text { Topoisomerase IV } \\
\text { inhibitor }\end{array}$ & $\begin{array}{l}\text { Mutations in gyrA and gyrB } \\
\text { causing an alteration to DNA } \\
\text { Gyrase A/B binding site (later } \\
\text { generations not always cross } \\
\text { resistant with first generation) and } \\
\text { increased ABC-type efflux pump } \\
\text { expression }\end{array}$ \\
\hline
\end{tabular}


Table 2-2. (continued)

\begin{tabular}{|c|c|c|}
\hline Drug Name & Mechanism of Action & Mechanism of Resistance \\
\hline Ethionamide & $\begin{array}{l}\text { Mycolic acid } \\
\text { biosynthesis inhibition }\end{array}$ & $\begin{array}{l}\text { Mutations in ethA and inhA causing } \\
\text { decreased pro-drug activation and } \\
\text { InhA overexpression (cross resistance } \\
\text { with Isoniazid) }\end{array}$ \\
\hline Cycloserine & $\begin{array}{l}\text { Peptidoglycan } \\
\text { biosynthesis inhibition }\end{array}$ & $\begin{array}{l}\text { Overexpression of alr } A \text { decreasing } \\
\text { drug efficiency }\end{array}$ \\
\hline Clofazimine & $\begin{array}{l}\text { Release of Reactive } \\
\text { Oxygen Species (ROS) } \\
\text { and cell membrane } \\
\text { disruption }\end{array}$ & $\begin{array}{l}\text { Mutation to Rv0678 causes } \\
\text { upregulation of MmpL5, a } \\
\text { multisubstrate efflux pump (cross } \\
\text { resistance with Bedaquiline) }\end{array}$ \\
\hline Linezolid & $\begin{array}{l}\text { Protein synthesis } \\
\text { inhibitor ( } 50 \text { S subunit) }\end{array}$ & $\begin{array}{l}\text { T460C mutation in } r p l C \text {, encoding the } \\
50 \mathrm{~S} \text { ribosomal L } 3 \text { protein and possible } \\
\text { efflux mechanisms }\end{array}$ \\
\hline $\begin{array}{l}\text { B-lactam/ } \boldsymbol{\beta} \text { - } \\
\text { lactamase } \\
\text { inhibitor: } \\
\text { Amoxicillin } \\
\text { Meropenem } \\
\text { Imipenem }\end{array}$ & $\begin{array}{l}\text { Cell wall disruption via } \\
\text { peptidoglycan } \\
\text { modulation }\end{array}$ & $\begin{array}{l}\text { Overexpression of } \beta \text {-lactamases, } \\
(B l a C) \text {, point mutations at target site } \\
\text { altering deacylation rate and binding } \\
\text { affinity, cell permeability (alteration in } \\
\text { porins and outer membrane } \\
\text { composition) and increased efflux } \\
\text { (Rv0194) }\end{array}$ \\
\hline Thiacetazone & $\begin{array}{l}\text { Inhibits } \\
\text { methyltransferases in } \\
\text { mycolic acid } \\
\text { biosynthesis }\end{array}$ & $\begin{array}{l}\text { eth } A \text { mutation minimizes pro-drug } \\
\text { activation and mutations to had } A B C \\
\text { operon affecting dehydratase activity }\end{array}$ \\
\hline Clarithromycin & $\begin{array}{l}\text { Protein synthesis } \\
\text { inhibition ( } 50 \mathrm{~S} \text { subunit) }\end{array}$ & $\begin{array}{l}\text { Low cell wall permeability and the } \\
\text { expression of emr } 37 \text {, confers } 23 \mathrm{~S} \\
\text { rRNA site modulation }\end{array}$ \\
\hline Bedaquiline & $\begin{array}{l}\text { Inhibition of } \\
\text { mitochondrial ATP } \\
\text { synthase }\end{array}$ & $\begin{array}{l}\text { atp } E \text { mutations introduces binding site } \\
\text { modulation. Noted efflux via mmpL5 } \\
\text { (cross resistance with Clofazimine) }\end{array}$ \\
\hline Delamanid & $\begin{array}{l}\text { Mycolic acid } \\
\text { biosynthesis inhibition }\end{array}$ & $\begin{array}{l}\text { Mutation of reductive activating } \\
\text { Rv3547 gene }\end{array}$ \\
\hline
\end{tabular}


As discussed previously, the accessibility of whole genome sequencing and genetic technologies that can be used to modulate gene expression levels has revolutionized our understanding of Mtb. The ability to search the genome for suitable therapeutic targets, to look for target conservation across multiple strains and lineages of Mtb and the ability to compare tractability with similar targets in other bacteria now provides a rich pool of information at the start of any discovery program.[161, 165-168]. It is now usually required that any new target based drug discovery programs are first validated by gene knockout and/or gene knockdown experiments [169, 170] to help ensure the druggability of the target. These experiments give key insight into the sensitivity of the Mtb cell to inhibition of a biochemical target, as it is clear that not all therapeutic targets are created equal and inhibition of some are more lethal than others.

The ability to screen Mtb under defined growth conditions that better represent the microenvironments of the Mtb life cycle is also an advancing technology in this field displaying great promise [171]. A good example is the high throughput screening of macrophage-resident $\mathrm{Mtb}$ that identified inhibitors active against cholesterol catabolism in Mtb [172]. Mtb is an intracellular pathogen that infects and multiplies in host macrophages, which is typically a very resource-deprived environment. In this case, the pathogen must scavenge carbon sources from the host, so Mtb has evolved specific catabolic enzymes to break down substrates like cholesterol and particle remnants of lowdensity lipids that are also scavenged by macrophages. Once inhibitors were identified in the macrophage based screen, the researchers tested for inhibition of cholesterol catabolic pathway (or methyl citrate cycle) inhibition by limiting the carbon source of in vitro grown cells in the presence of the inhibitor. These studies led to novel inhibitors of HsaAB, a two-component, flavin-dependent hydroxylase that is crucial to the degradation of the "A" ring of cholesterol [172]. While these inhibitors are still under development, they are best considered as chemical probes with which to study the role of cholesterol catabolism in Mtb infection. The Russell group's work is a landmark study because it has identified and validated a new area of Mtb metabolism for chemotherapeutic intervention and drug design.

Technology that enables us to detect and image drug distribution allows researchers to gain a better understanding of how TB drugs distribute within the lungs of patients and how this exposure compares to rodent models of Mtb infection is another significant recent advance. It is quite easy to look at a single parameter (e.g., plasma concentration) and be deceived about how a drug will act in a patient. However, plasma concentration may be a poor indicator of tissue and granuloma perfusion, especially perfusion into necrotic granulomas' caseum-filled cores [173]. This phenomenon could help explain the failure of the Rapid Evaluation of Moxifloxacin in Tuberculosis (REMoxTB) clinical trial, one of the largest Phase III TB trials ever conducted. The trial, which included more than 1,900 patients, was proposed to shorten the duration of Mtb therapy from 6 to 4 months by substituting moxifloxacin for either INH or EMB, was supported by the results from extensive combination testing in mice [174]. The trial was unable to confirm the treatment shortening potential of moxifloxacin seen in mice, and subsequent data has shown that moxifloxacin is not able to fully penetrate human caseous 
lesions that have a low degree of celluarity [173], despite showing high concentrations inside activated macrophages $[175,176]$.

These results have further spurred on efforts to study location-dependent drug concentrations in the Mtb infected lung, taking advantage of new MALDI-MS imaging technologies [175, 177-180]. In a small study of drug distribution in granulomas taken directly from the lungs of MDR-TB patients who were treated with drug combinations at varying time points prior to lung resection, moxifloxacin exhibited several-fold lower concentrations in caseous regions of pulmonary lesions compared to RIF, concentrating around the periphery of the lesion. RIF exhibited a slow accumulation until high steadystate concentrations were achieved deep within lesions, and PZA was seen to rapidly perfuse throughout the lesion. This inability to efficiently drug the entire lesion probably explains why shortening the course of REMoxTB trail was not successful. These findings support a previous in vivo study in rabbits that used similar matrix-assisted laserdesorption/ionization mass spectroscopy imaging [181, 182], effect. These results suggest that penetration into caseous lesions is critical for future efforts towards treatment shortening regimens (Figure 2-5).

\section{Chapter Outline}

This chapter will focus on two projects to identify novel drugs to combat drug resistant $M t b$ by targeting the cell wall. The first is targeting the clinically relevant FAS II pathway, the target of multiple currently used anti-tuberculars. Rationale design of new inhibitors at an essential enzyme in this pathway is used to develop compounds that are not subject to common resistance mechanisms and overcome poor drug-like properties that afflict second line agents that already inhibit the pathway. The second project is the optimization of a whole cell phenotypic screening compound to improve physical chemical properties. This compound series acts on a novel target, which is good for targeting MDR-TB. Both of these series highlight the strengths and weakness of two commonly implemented drug discovery techniques that are discussed at the end of the chapter.

\section{Fatty Acid Synthase II (FAS-II)}

$\mathrm{INH}$ is a staple of frontline tuberculosis therapy. Its molecular target is the mycobacterial enoyl-ACP reductase InhA, which is required for the biosynthesis of mycolic acids, the dominant feature of the lipophilic outer mycobacterial cell wall that is essential for growth and virulence. [183, 184]. There are two distinct fatty acid biosynthesis (FAS) routes; mammals rely on the FAS I pathway, and bacteria rely on the FAS II. Mycobacteria contain both pathways, with a distinct subset of enzymes included in FAS II for the biosynthesis of extraordinarily long mycolic acids. These FAS II enzymes are where drugs can be designed for mycobacterium specificity, an important consideration for the development of a therapy for MDR-TB. FAS-II is responsible for the synthesis of the $\mathrm{C}_{56}$ fatty acids that provide the meromycolate backbone that is 


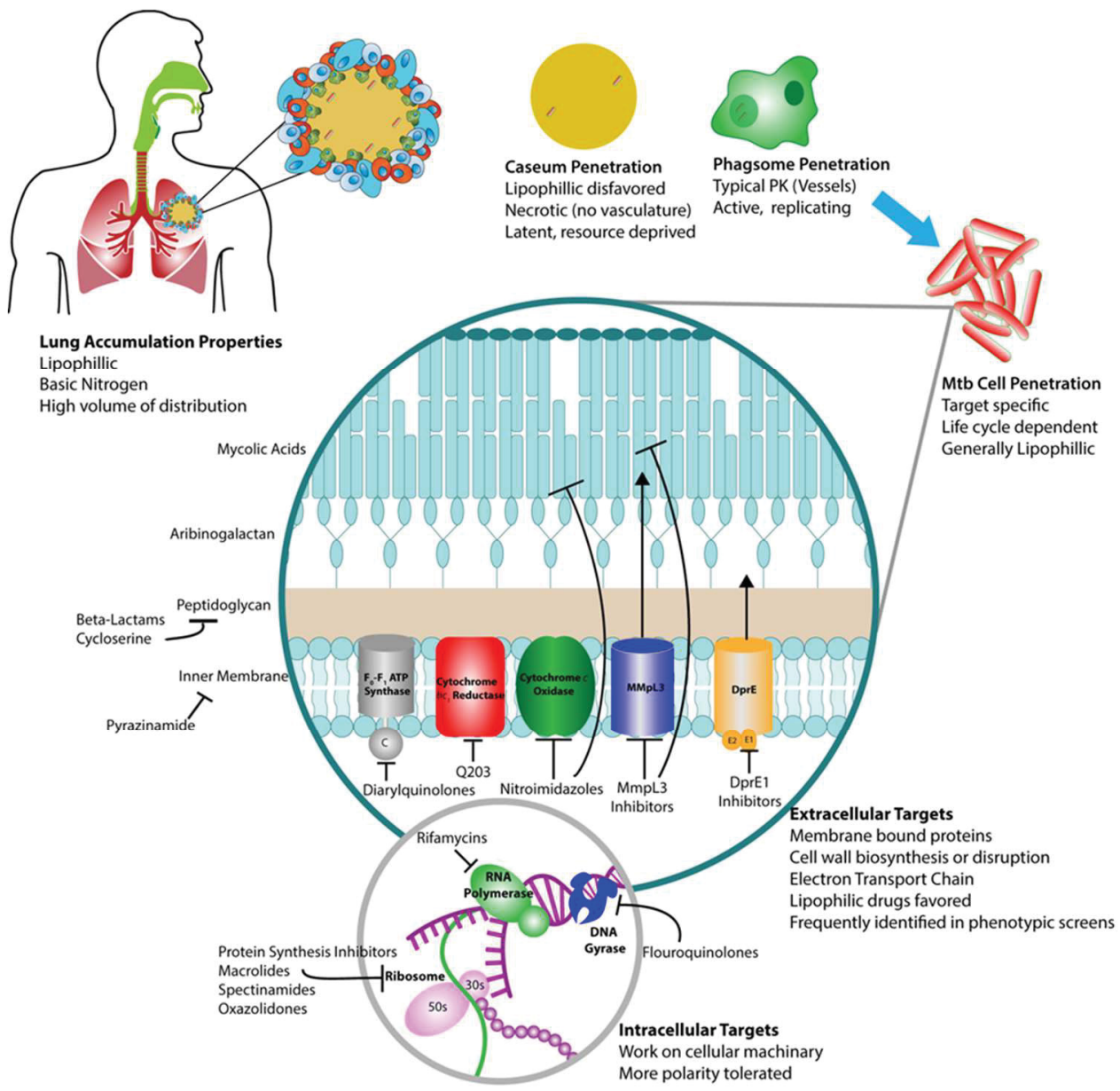

Figure 2-5. The various environments a drug must thrive in to be an effective antitubercular agent

Reprinted with permission from Elsevier Science. Hoagland, Daniel T et al (2016). "New Agents for the Treatment of Drug-Resistant Mycobacterium Tuberculosis." Advanced Drug Delivery Reviews 102(1): 55-72 
condensed with the product of FAS I to form mycolic acids. Mycolic acids are homologous series of 60-90 carbon $\alpha$-alkyl $\beta$-hydroxy fatty acid that are present in all mycobacterium and are one of the cells best intrinsic defenses from immune and xenobiotic threats. INH is a prodrug that requires activation by the catalase-peroxidase KatG to inhibit InhA, and this bioactivation step is where Mtb primarily develops resistance by inactivating this nonessential enzyme (Figure 2-6). Other drugs such as ethionamide and thiacetazone inhibit distinct enzymes in this pathway and are also bioactivated by non-essential mechanisms (monooxygenase EthA). The resulting INHNAD adduct inhibits the enzyme and displays remarkable residency time due, in part, to a conformational shift in helix 6 that occurs after the initial binding, a shift that does not occur with rapid reversible inhibitors of InhA [185-187]. Peter Tonge and others have stressed the importance of residency time in drug discovery and lead optimization [188190]. Just having tight binding affinity is not enough. Typically, it is the dissociation rate of the inhibitor from the enzyme that is more indicative of the biological activity in vivo [191]. The pathway is a sequence of initiation via the carboxylation by FabH of acyl-CoA produced in FAS-I. The resulting 3-ketoacyl-ACP is reduced by MabA to the hydroxyl from where it's dehydrated by HadAB/BC heterodimer complexes. The enoyl produced by this dehydration is the substrate for InhA where the elongated ACP can be desaturated and modified by various methyltransferases before the final claisen condensation with the $\alpha$-branch from FAS I performed by Pks13 (FadD32) [192]. Fatty acid biosynthesis offers a tremendous number of drug targets and is a commonly targeted pathway for both clinically used agents and those under development.

\section{Introduction: HadAB-Known Inhibitors and Pharmacology}

The dehydratase portion of the Mtb FAS II cycle is carried out by a pair of heterodimeric protein complexes, HadAB and HadBC, that are encoded by the essential had $A B C$ gene. It has been known for some time that drugs like ISO and TAC inhibit mycolic acid biosynthesis, but it has been demonstrated recently that their exact target was this essential dehydratase process $[193,194]$. Spontaneous mutants were found to have changes at the HadA and HadC locations and overexpression of HadABC resulted in a 16-fold increase in whole cell MIC levels. Through biochemical and genetic testing it was determined that HadAB acted early in the elongation process while HadBC worked on much longer fatty acyl substrates making this subunit only required for virulence while HadAB is required for growth [195]. The exact mechanism of how ISO and TAC inhibit HadAB has recently been shown to be covalent in nature. Both undergo bioactivation by the flavin-dependent monooxygenase EthA to a sulfinic acid where a nucleophilic attack by a non-catalytic cysteine occurs forming a disulfide bond (Figure 2-7). From here a distal cysteine residue displaces the drug forming an inactive cross linked protein and releasing free drug. Herein, we describe work to identify and optimize a new generation of inhibitors targeting the essential Mtb dehydratase HadAB, the validated bactericidal target of ISO and TAC. To accomplish this we use structure guided design of reversible covalent inhibitors that removes the requirement for prodrug activation and mitigates toxicity, fragment-based drug design approaches and whole cellbased screening. Once hits are identified they will coupled to an iterative medicinal 

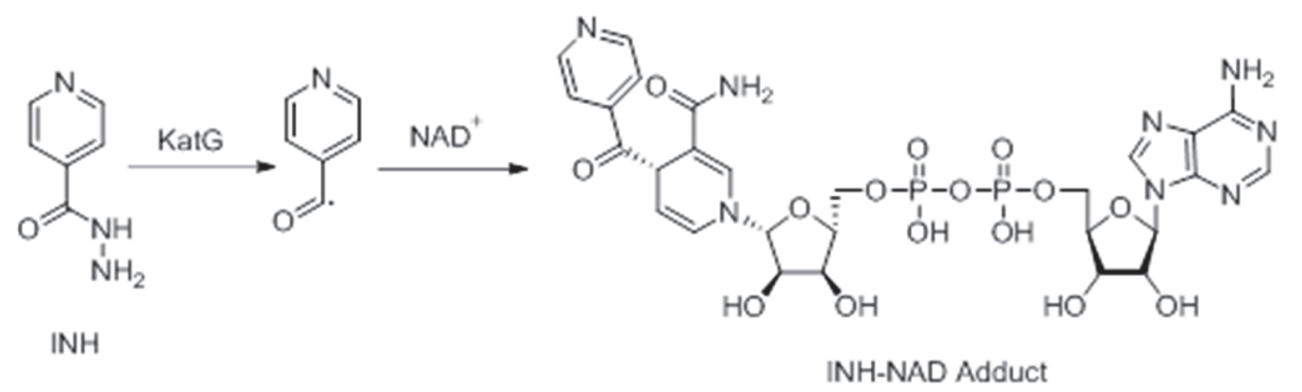

Figure 2-6. Mechanism of INH bioactivation by KatG and forming a covalent adduct with NAD to inhibit InhA

Reprinted with permission from Elsevier Science Hoagland, Daniel T et al (2016). "New Agents for the Treatment of Drug-Resistant Mycobacterium Tuberculosis." Advanced Drug Delivery Reviews 102(1): 55-72 


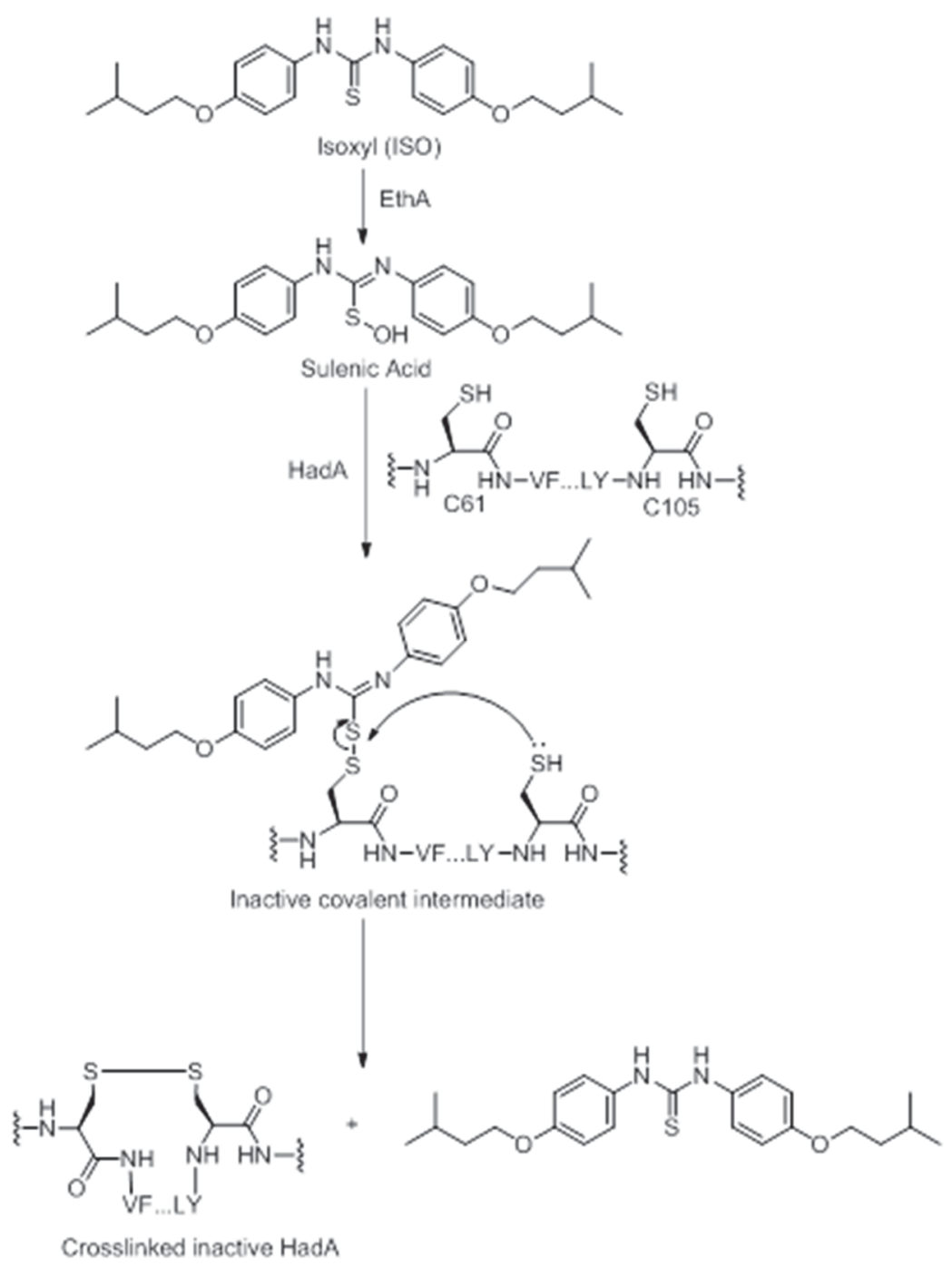

Figure 2-7. Mechanism of ISO's bioactivation to a sulfenic acid Able to inactively crosslink two cysteine residues of HadA 
chemistry, biological and pharmacokinetic optimization of emerging leads to ultimately produce potent dehydratase inhibitors showing activity in vivo.

\section{Reversible Covalent Inhibitors of HadAB}

The medicinal chemistry field of covalent inhibitors has recently been boosted with the approval of Ibrutinib [196]. Ibrutinib is a first-in-class Bruton's tyrosine kinase (Btk) irreversible covalent inhibitor for the treatment of chronic lymphocytic leukemia (CLL) and other B-cell malignancies. Ibrutinib is highly selective for Btk ( $>1000$ fold selectivity by $\mathrm{IC}_{50}$ vs other kinases) and is able to fully occupy the Btk active site at just $10 \mathrm{nM}$. Such selectivity and potency is critical in its approval since it can be dosed quite low and still have dramatic effects. Covalent inhibitors have traditionally been stigmatized in drug discovery, due to their propensity for off target toxicity and selectivity profiles [197]. However in the past few years there has been a dramatic increase in developing covalent inhibitors with a high degree of selectivity like ibrutinib that can illicit similar efficacy and incredible residency time. It has been demonstrated that Btk inhibition could have promise in collagen-induced arthritis and other forms of autoimmune inflammation $[198,199]$. As selective as ibrutinib is, it still possesses off-target kinase and non-kinase binding that limits its dosing amount and duration, limiting it from this indication [200].

The Taunton group has worked on new approaches to target non-catalytic cysteine residues near the active sit, developing semi-covalent reversible inhibitors of the Btk around the scaffold of ibrutinib [201] (Figure 2-8). The group was able to successfully demonstrate tunable target residency time and inhibition, dependent on functionalization to a nitrile activated acrylamide warhead group. This reversible attachment is crucial for eliminating off-target covalent adducts such as with glutathione, a cause of concern in most covalent modifiers. There is still a high degree of site specificity built into the design of these kinase inhibitors, which primarily drives potency. The primary purpose of the acrylonitrile group is to ability to modulate residency time at the target, in such a way to balance the pharmacodynamics of inactivation of dehydratase enzyme through crosslinking of the cysteine. The elucidation of ISO and TAC's mechanism of action has opened up the possibility to develop other compounds that act covalently to modify the non-catalytic cysteine near the active site. As such, a series of ISO mimics were designed and synthesized to achieve three goals: replace the thiourea found in ISO and TAC, maintain a covalent modifying group to target HadAB C61 while mitigating off target binding and do so in a way that doesn't require bioactivation that would confer cross resistance with other FAS-II inhibitors.

\section{Fragment-Based Drug Discovery}

In the early 2000's antibiotic drug discovery was dominated by high throughput screening (HTS) approaches. Academics and industry alike would screen hundreds of thousands of drug-sized molecules (molecular weight $>300 \mathrm{Da}$ ) either against a specific target, or just to test for cell death. However, target based screening proved to be largely 

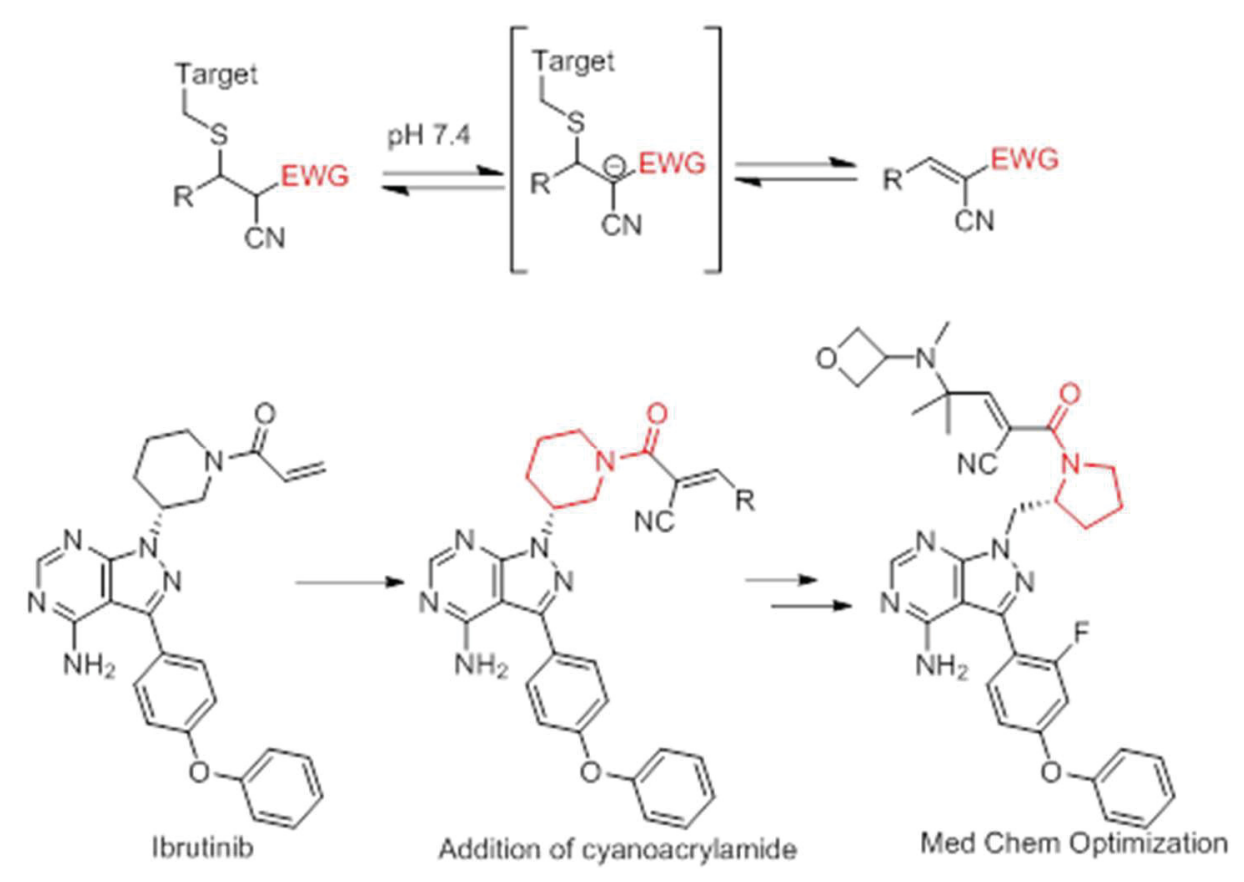

Figure 2-8. Medicinal chemistry optimization of ibrutinib into a semi-covalent reversible acrylonitrile for residency time studies 
ineffective at transitioning between in vitro activity to whole cell and in vivo efficacy. Phenotypic screening also was not productive in the discovery of new chemical matter, often suffering from poor physical chemical properties. One of the drawbacks in HTS is the ability to properly probe chemical space. A normal HTS library has on the order of $10^{6}$ compounds, but taking into account just commonly associated heavy atoms (HA) there are upwards of $10^{60-200}$ drug-sized compounds possible leaving this approach woefully short in exploring chemical space [202]. Using much smaller molecules $(<11$ HA) it is only possible to generate $10^{7}$ combinations, getting a much better sampling of available chemical matter. Generally most fragment libraries adhere to a Rule of Three: molecular weight $<300 \mathrm{Da}$, up to three hydrogen bond donors, up to three hydrogen bond acceptors and a calculated $\log$ patrician coefficient $(\mathrm{cLogP})$ of $\leq 3$ [203]. These much smaller molecules are also less likely to have intrinsic steric interference with the binding site and a higher probability of a pharmacophoric match. A large molecule may have a portion of its structure that is suitable for binding but another feature could strongly disfavor it, meaning it is lost as a negative in traditional HTS. However these smaller molecules do not typically have enough binding capabilities to elicit a biological response in a whole cell system. Sensitive biophysical or biochemical assays such as surface plasmon resonance (SPR) are required to determine binding affinities and rank order screening hits. After screening the typical workflow would be to use structure guided design to rationally develop analogs and up binding affinity. Useful metrics employed to monitor the growing fragment are ligand efficiency (LE) $(\Delta \mathrm{G} /$ Heavy atom count) and ligand-lipophilic efficiency (LiPE) (pIC50- cLogP), which links potency to the cLogP to estimate a compound's drug likeness. After several iterations of medicinal chemistry an optimized inhibitor will be closer to a lead-like molecule produced from an HTS in terms of drug like properties, with high target specificity. We describe efforts to use our curated library of 4,500 well behaving fragments to establish binding affinities for molecules at the HadAB complex in the hopes of development into inhibitors on their own or for addition of the previously described reversible covalent attachment point.

\section{Results}

The synthesis of acrylonitrile Michael acceptor group is straightforward and modular, excellent for diversity oriented synthesis (Figure 2-9A). 11 analogs were first synthesized to mimic half of ISO's symmetric structure by coupling of alkyl halides to 4hydoxybenzaldehyde and combination with various 3-oxopropanenitriles with triethylamine and microwave conditions. The other half of the molecule served to install small electron withdrawing groups in the hopes of both filling the space occupied by the second benzene ring. This synthesis is open to a wide variety of aldheydes and propanenitiriles to generate a large number of compounds, as well as is adaptable enough to anchor this acylonitriles to optimized fragments. A brief covalent docking study was conducted to design a secondary series of more drug-like side chains (Figure 2-10). ISO is notorious for poor solubility, undoubtedly a product of a very high lipophillicity $(\mathrm{cLogP}=6.23)$. After a quick search of commercially available aldehydes, iterative ligands were generated and docked using the CovDock module in the Schrödinger software pack. CovDock first docks the ligands in to a receptor where the reactive residue 

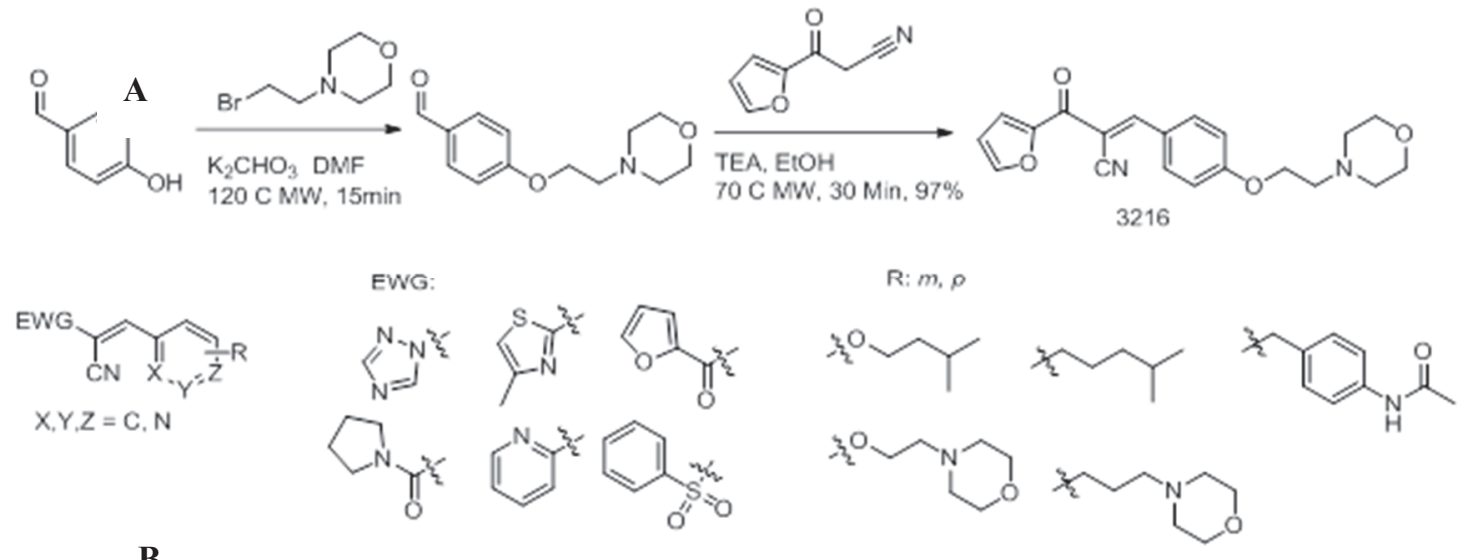

Figure 2-9. Design of HadAB inhibitors

A. Schematic of the two step synthesis to achieve these acrylonitrile inhibitors. B.

Planned analogs designed to modulate electron withdrawing and drug-like properties of top inhibitors.
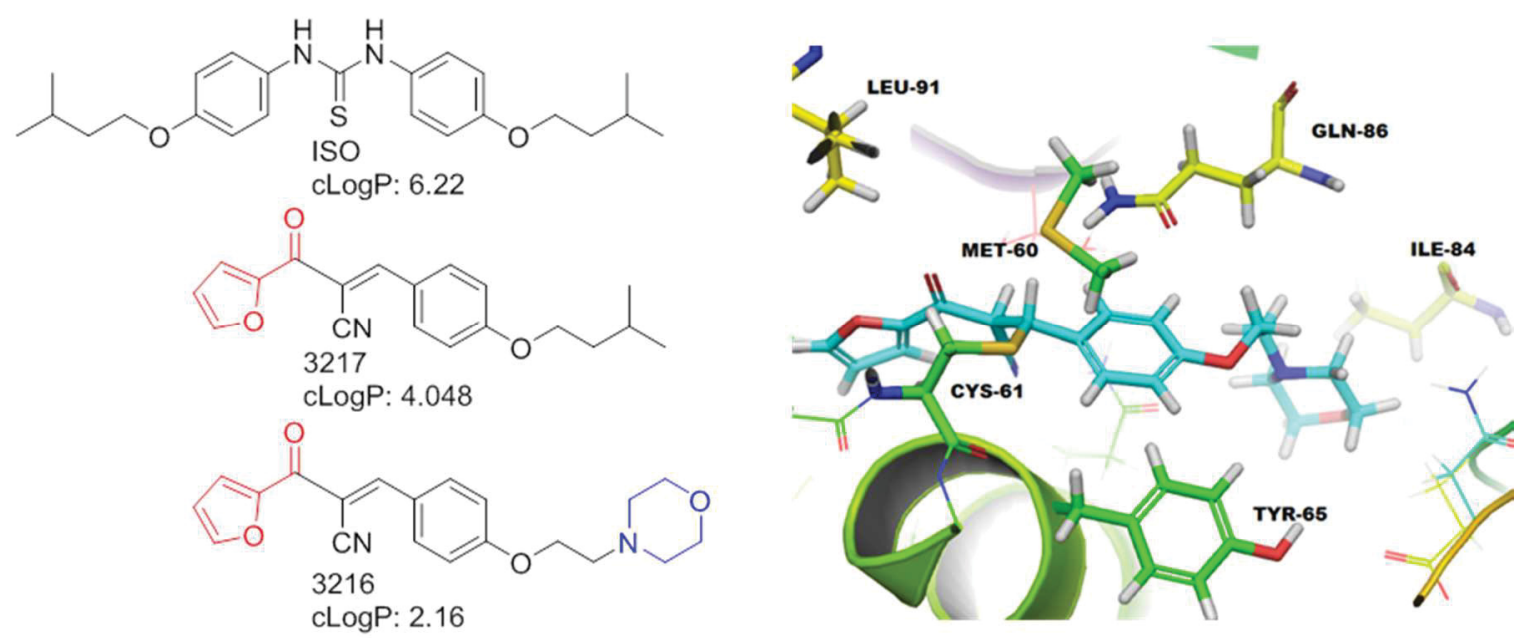

Figure 2-10. Rational development of more drug-like ISO mimics

That contain the acrylonitrile functional group. In red reflects the election withdrawing side group and in blue the replacement morpholine ring system chosen from covalent docking studies shown on the right. 
has been mutated to an alanine. The reactive residue is then switched back and sampled to form a covalent bond with the ligand in different poses. Covalent complexes are minimized and scored to determine the top covalent complexes. An apparent affinity score, based on the docking score of pre-reactive and post-reactive poses, is also calculated to estimate binding energies for use in virtual screening. The best scoring series was one that contained a morpholine group; the tertiary nitrogen was predicted in several poses to form a hydrogen bond with T65. Swapping this side chain for the branched alkyl chain of ISO dropped the cLogP of $\mathbf{3 2 1 7}$ from 4.05 to 2.16 in $\mathbf{3 2 1 6}$ whileactually increasing its MW by 43 Da (Figure 2-10). This could have a dramatic effect on efficiency metrics that are becoming more popular in drug discovery such as Lipophillic ligand efficiency (LiPE) which links potency to the cLogP to estimate a compound's drug likeness. In general it is much easier in medicinal chemistry to add lipophilic groups, and as such screening hits almost always see their lipophilliticy raised in optimization.

Enzymatic activity was monitored in two fashions: using an absorption based readout and an NMR reaction monitoring program, both meant to monitor the rate of substrate processing by the enzyme. The absorption assay is a spectrophotometric enzymatic assay developed by Sacco et al to determine the ability of the protein complex to transform substrate [194]. $(R)$-specific enoyl hydratase/hydroxyacyl dehydratase family enzymes have been shown to preferentially catalyze the hydration reaction when isolated from their enzymatic complex kinetic assays in the presence of trans-2-C12:1$\mathrm{CoA}$ were monitored at $263 \mathrm{~nm}(\Delta \mathrm{A}$ of 0.67 for a variation of $100 \mu \mathrm{M}$ in substrate concentration). We have also characterized the HadAB functionality using an NMR reaction monitoring system. Incubating substrate with protein allows us to visualize the change in the chemical shifts associated with the hydroxylation of the unsaturated bond in the fatty acid side chain. The addition of inhibitor to the protein before the addition of the substrate allows us to visualize a lack of transformation, or at least a slowing of the rate. Ebselen is a selenium based compound commonly used in covalent bond studies, and has been shown to covalently inhibit the mycobacterium antigen 85 complex at a similar cysteine residue. This led us to use ebselen as a control compound in hopes that it formed a similar covalent bond with $\mathrm{C} 61$. We were able to obtain a crystal structure of

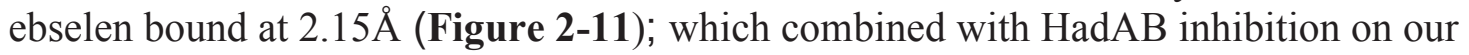
spectrophotometric assay gives us confidence that this is a useful control compound to screen other potential inhibitors. We have demonstrated that multiple reversible covalent inhibitors are able to slow this reaction considerably both compared to no treatment and ebselen control, giving us confidence that we are on target in our synthetic rationale to design this novel class of anti-tubercular compounds (Figure 2-12).

Compounds like $\mathbf{3 2 1 3}$ and $\mathbf{3 2 1 6}$ contained the morpholine ring that was prioritized due to the CovDock studies and were able to slow the reaction rate more than their simple branched alkyl chain analogs (3214 and 3217 respectively Figure 2-13). Extension beyond 5 atoms past the unsaturated bind with a sulfonyl resulted in four inactive compounds relative to DMSO control (Figure 2-14), 3187, 3189 and 3210 actually significantly increasing the rate of hydroxylation. This could imply activation of the enzyme, but certainly requires more extensive investigation before making any 


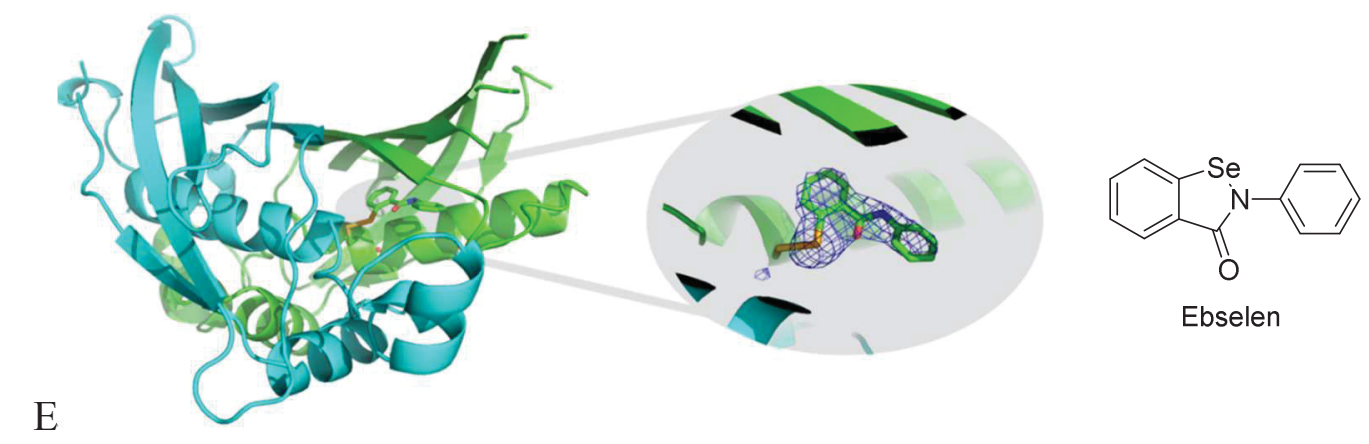

Figure 2-11. Ribbon diagram of the HadAB heterodimer co-crystal structure with ebselen

HadA in green and HadB in cyan. Ebselen shown covalently bound to C61. Crystal captured at $2.15 \AA$

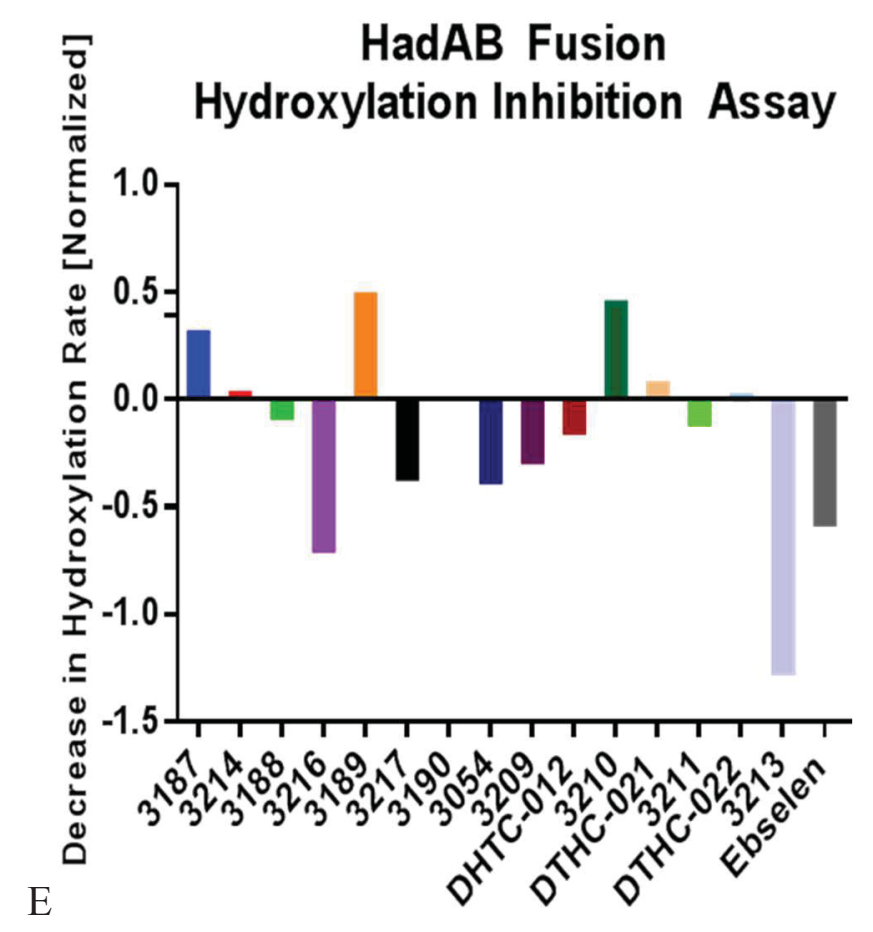

Figure 2-12. Spectrophotometric inhibition assay

Used to measure HadAB enzyme activity using a trans-2- $\mathrm{C}_{12: 1}-\mathrm{CoA}$ substrate and monitoring absorbtion at $263 \mathrm{nM}$ with $100 \mu \mathrm{M}$ of inhibitors. Rates normalized to DMSOonly control and ebselen used as a positive control. 

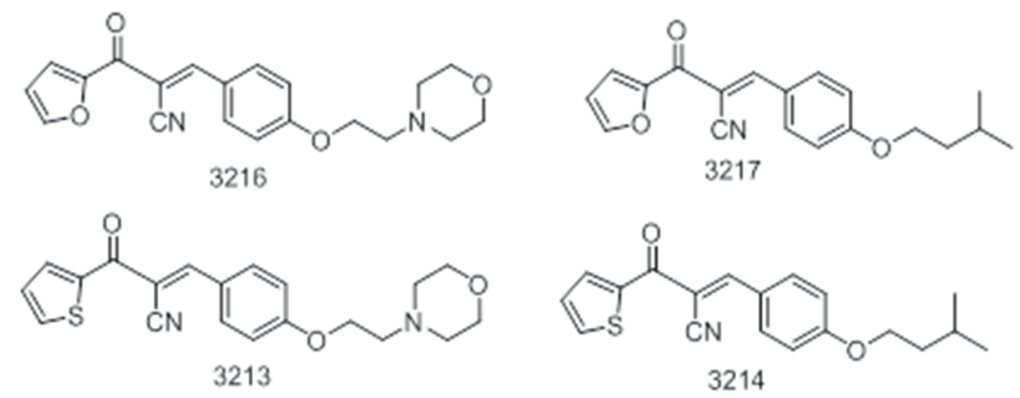

Figure 2-13. Four of the best acrylonitrile compounds

Highlight the advantage gained in the inhibition assay by the addition of the morpholine ring.
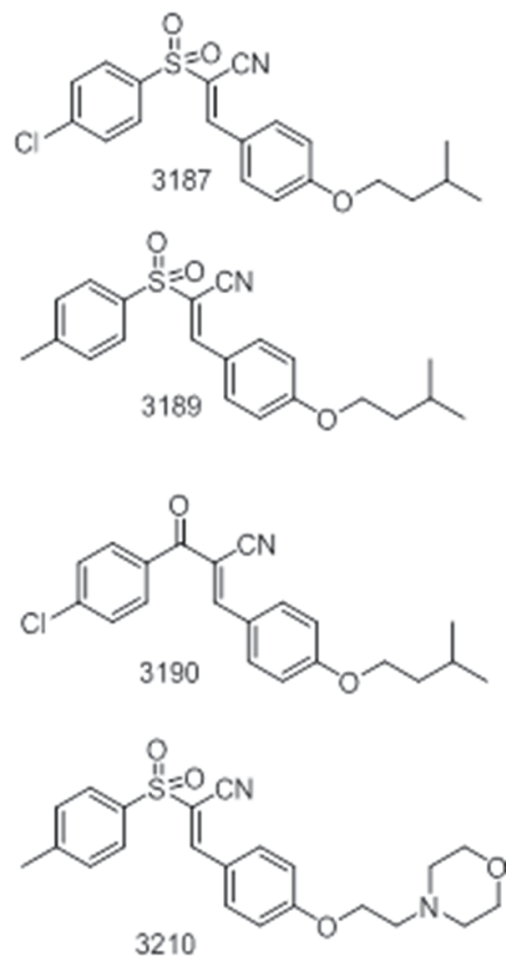

Figure 2-14. Four poor acrylonitrile inhibitors

Demonstrate extension beyond 5 atoms is deleterious to inhibition using sulfone and carbonyl as the electron deficient linker and both studied side chains. 
claims. Interestingly the substituted phenylsulfones (3188 and 3209, Figure 2-15) which only extend five atoms past the unsaturated bond are modest inactivators where the morpholine ring is still the more potent side group. Analogs are planned to harness more amide linkages and more diverse heterocyclic groups to further modulate polarity and proton affinity (Figure 2-9B), but more precise assays are required to generate concrete enzymatic SAR analysis. We have performed a ligand detection, reaction monitoring NMR based assay that collaborates our absorption based findings for the reversible inhibitors (Figure 2-16). Protein was incubated with substrate and we were able to gauge reaction kinetics using MestReNova's reaction monitoring software. We tracked the chemical shifts associated with protons labeled $\mathbf{1}$ and $\mathbf{2}$ in Figure 2-16C, and monitored their transfer upfield as the substrate became hydroxylated (Figure 2-16A). We demonstrate a protective effect upon pre-incubation of the HadAB protein with $100 \mu \mathrm{M}$ of 3216 (Figure 2-16B) and after 45 minutes there is still limited hydroxylation, especially compared to just protein and substrate alone (Figure 2-16A). There are corresponding upfield shifts in the aliphatic region (ppm 4.0 and 2.7, not shown) in unprotected protein that correlate to hydroxylation of the substrate that do not appear in pre-incubated sample.

At St Jude we have access to a library of 4,500 fragments from commercial sources, pre-screened to remove promiscuously destabilizing compounds and those which interfere with assay conditions. Using purified HadAB, SPR binding studies were conducted to register affinities and off rates for these molecules. SPR is the preferred primary screening method due to its amenability to be both high throughput as well as highly sensitive for weaker affinity compounds that we expect these fragments to be. We have identified 63 hit molecules (50\% of control response) and 12 fragments with $\mathrm{K}_{\mathrm{D}}$ 's under $100 \mu \mathrm{M}$. Going forward with medicinal chemistry, scaffolds will be prioritized using lipophilic ligand efficiency metrics discussed previously using our binding affinity $\left(\mathrm{K}_{\mathrm{D}}\right)$ as our potency marker. The lab has used this technique successfully in optimization processes for HTS screening [204] and we believe it can be a powerful guiding tool for fragment based drug discovery. Looking at Figure 2-17 we discuss how we will reprioritize our hits early based on structure and lipophilic efficiency. Currently one would select chemically diverse series with the best binding affinity (i.e 1-3). However 2aminothiazoles like $\mathbf{1}$ are known to be a promiscuous inhibitors across many screens [205] and have been nicknamed PrATS (Promiscuous 2-AminoThiazoles). As such it will be treated with trepidation and has a highly likelihood of being dropped early. Moving the discussion back to efficiency, normally 2 would be prioritized over $\mathbf{3}$ based on affinity, however based on LiPE $\mathbf{3}$ would be the top choice. These rules are not completely rigid, as removal of the chlorine atom from $\mathbf{2}$ could yield a similarly potent fragment with a dramatically reduced $\operatorname{cLogP}(3.88 \rightarrow 2.0)$ and thus much higher LiPE. A better example of prioritization would be looking at compounds 4-6. 4 and 5 are much tighter binders than $\mathbf{6}$, but their high degree of lipophillicity relative to $\mathbf{6}$ would score them much lower than the modest binder. These modest binders are easier to build out for potency while maintaining favorable properties associated with a high LiPE. It cannot be understated that the use of one efficiency metric can be problematic, looking at multiple data sources will always give a more complete understanding. 


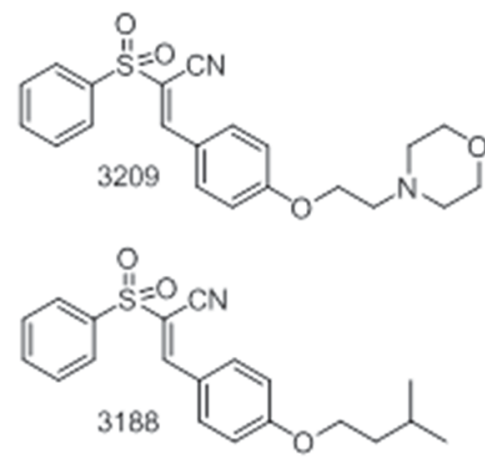

Figure 2-15. 3209 and 3188 regain activity by the deletion of one atom beyond the phenyl ring system 

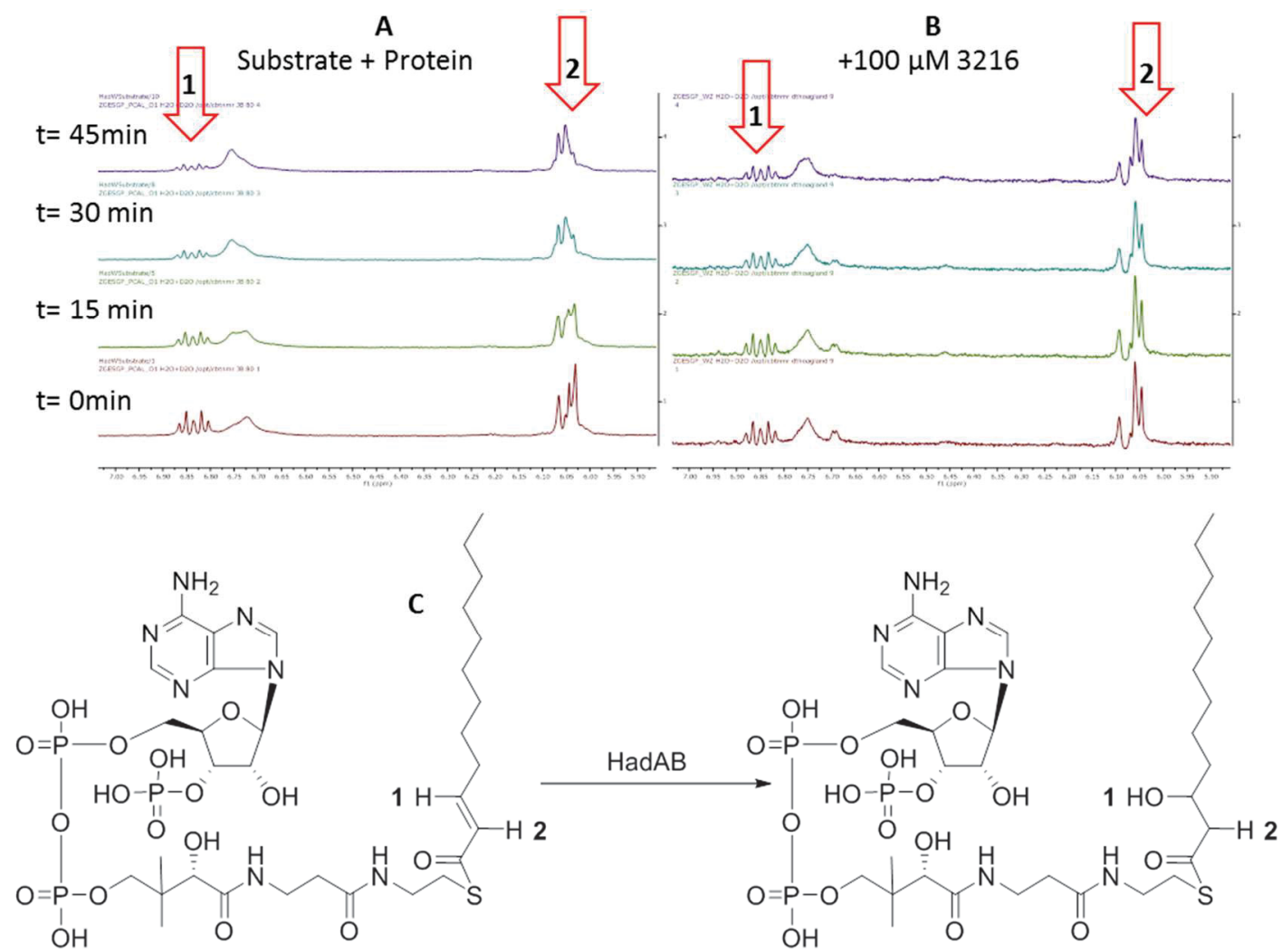

Figure 2-16. Ligand detected ${ }^{1} \mathrm{H}$ NMR study

NMR based substrate processing assay. Reaction monitoring software tracked the spectral shifts of positions $\mathbf{1}$ and $\mathbf{2}$ as hydroxylation occurred (A). Corresponding shifts occur upfield (not shown) and there is a demonstrative protective effect by 3216 (B) for up to 45 minutes. 


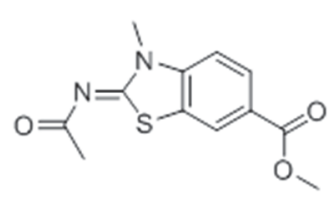

$K_{D}=15.4 \pm 0.2 \mu \mathrm{M}$

$\mathrm{LE}=0.37$

cLogP: 3.662

LiPE $=1.15$

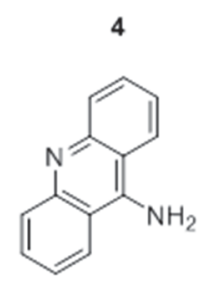

$K_{D}=122 \pm 1 \mu \mathrm{M}$

$\mathrm{LE}=0.36$

CLogP: 3.086

LiPE $=0.83$

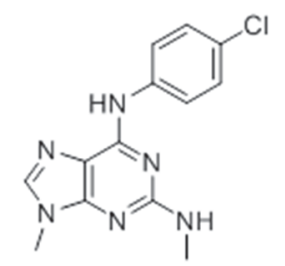

$K_{D}=21 \cdot 4 \pm 0.1 \mu \mathrm{M}$

$\mathrm{LE}=0.32$

cLogP: 3.87994

$\mathrm{LiPE}=0.95$

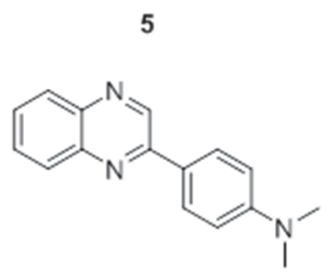

$K_{D}=192 \pm 2 \mu M$

LE $=0.27$

CLogP: 3.69323

LiPE $=0.02$
3

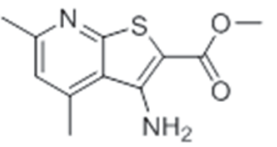

$K_{D}=62 \pm 1 \mu \mathrm{M}$

$\mathrm{LE}=0.36$

CLogP: 3.17683

LiPE $=1.03$

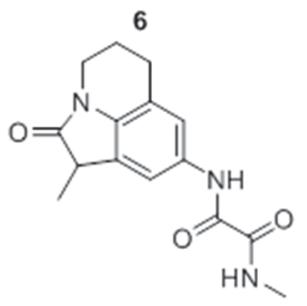

$\mathrm{K}_{\mathrm{D}}=382 \pm 4 \mu \mathrm{M}$

LE $=0.22$

CLogP: -0.344999

$\mathrm{LiPE}=3.76$

Figure 2-17. Representative fragment hits used to discuss the application of lipophilic ligand efficiency as a filter to select scaffolds to pursue going forwards. 
X-ray crystallography conditions are being optimized to verify the binding modes of these fragments for structure guided medicinal chemistry. Not unexpectedly, none of the fragments made a significant enough interaction with the protein to illicit any distinguishable rate change in our enzymatic assays. We are also looking into the binding mechanisms of a small library of covalent fragments recently purchased. These fragments will be biased to bind near our residue of interest which should give more structural information about binding pocket itself. Ultimately the idea is merge tight binding fragments to our reversibly covalent functional group approaches to generate site specificThese molecules will then be further optimized to obtain and maximize MIC and pharmacokinetic properties.

\section{Discussion}

Fatty acid biosynthesis is a tantalizing drug target for drug resistant tuberculosis. There are multiple enzymatic targets that are previously validated as clinically relevant anti-tuberculars and ample opportunity to mitigate common resistance mechanisms while developing more effective drugs. The recent discovery of a naturally occurring InhA inhibitor gives us even more confidence that the FAS-II pathway is not only druggable, but in fact evolutionarily critical as an antibiotic target [206]. The results we have generated give us hope that we can design a highly specific reversibly covalent inhibitor of the HadAB enzyme that can be mycobacteria specific with limited off target events. With on-going crystallography studies guiding medicinal chemistry we hope to learn a great deal about the binding mechanics required to have potent inhibition without the need for a long lasting covalent bond. We need to develop an optimized substrate with a longer aliphatic chain to gain more sensitivity in our inhibition to delve into proper dose response. The current substrate is not optimized for HadAB, the endogenous substrate has a much longer aliphatic chain giving it a much higher affinity. However outside of the cellular environment these longer chain analogs lose solubility that renders these assays suspect. We are working on the production of longer chain ACP analogs that may have higher specificity and provide a much more sensitive biochemical assay. We are currently developing a native state mass spectrometry method of detecting binding, a technique that is gaining traction in the fragment drug discovery field [207,208]. We have had difficulties establishing binding affinities of our reversible inhibitors, due to complexation with the chemical matrix used to immobilize the protein. This technique has proven capable of working in concert with high throughput biophysical techniques such as SPR, and has good track record of predicting molecules that will form co-crystal structures [207]. Adding a secondary biophysical screen will help in prioritizing compounds to be sent for these crystallography studies and should allow us to gauge relative affinities [208]. Structure guided design will be invaluable to the continued optimization of this series. These inhibitors will bypass the most common resistance mechanism in targeting FAS-II, the requirement of bioactivation by the mycobacterium. In fact, the covalent modifier's activity should be primarily driven by normal binding enthalpy in its hydrogen bond and hydrophobic interactions with the protein. This could actually help these molecules avoid both of ISO and TAC's common resistance, as mutations at the $\mathrm{C} 61$ position might not diminish their affinity enough to overcome any 
associated fitness cost. It certainly is an intriguing prospect that this project will continue to push towards.

In addition to target guided design of new inhibitors we are also working on identifying HadAB inhibitors from known anti-tubercular compounds using whole cell phenotypic screening. Using a library of 1,500 known anti-tubercular compounds provided by Global TB Alliance, collaborators have overexpressed HadAB to find a drug whose MIC shifts. This approach harnesses the advantages intrinsic to phenotypic screening while accounting for our target of interest; it accounts for cellular penetration and efflux by requiring an active molecule to get to the target of interest to actually illicit its effect. Drug screening will be discussed at length in the next chapter, as will the advantages of repurposing known bioactives that have extensive preclinical data available already.

As we learn more about the function and role of this protein complex and how potential inhibitors behave in this environment we can begin to have a better grasp of the most effective way to use this target clinically. Ideally a novel anti-tubercular drug will have a unique mechanism of action to minimize cross resistance, which we can achieve here by circumventing bioactivation. Mechanistically there is potential for synergy by targeting two targets in the FAS-II system. INH forms a covalent adduct with NAD that binds tightly to InhA and acts as a competitive inhibitor. By out-competing the natural enoyl-ACP, INH is acting in a similar fashion as SMX. SMX out competes PABA for conjugation to DHF, which removes this metabolic precursor from the pathway making the bacteria work harder to produce its co-factor. When TMP is introduced there is marked synergy because there is less DHF for TMP to compete for binding at the DHFR. It could be a good investigation to look at the potential synergy of co-administration of FAS-II inhibitors upstream of InhA to see if similar metabolic depletion could aid in efficacy. Similar studies with current HadAB inhibitors would be difficult due to their toxicity concerns and common bioactivation pathway, EthA, which activates 3 of the existing FAS-II inhibitors (Ethinoamide, ISO and TAC) making combinations highly susceptible to resistance. The success of this project could lead into very interesting studies with the potential to drastically alter MDR-TB therapy for the better.

\section{MmpL3 as a Drug Target}

\section{Function and Druggability}

The Mycobacterial membrane protein Large (MmpL) is a family of export proteins involved in transportation of metabolites from the cytosol of Mtb. The Mtb genome contains 12 genes that express the MmpL proteins that are considered resistancenodulation-division proteins, which play an important role in Mtb survival and pathogenesis [209]. MmpL3 is required for the export of mycolic acids in the form of trehalose monomycolates to the periplasmic space or outer membrane. Established as the only protein in this family that is essential for mycobacterial survival, MmpL3 is an 
attractive drug target. It is an important membrane protein, and a very common target elucidated in hits from whole-cell phenotypic assays, similar to DprE1 and QcrB [210]. Because MmpL3 is an easily druggable target with proven essential roles in Mtb survival, there is immense interest in MmpL3 inhibition as a novel mechanism of action for a new anti-tubercular agent.

There are a host of compounds that have been found to elicit their anti-tubercular activity via inhibition of MmpL3 (Figure. 2-18). The most advanced MmpL3 inhibitor is SQ109, a structural derivative of EMB's diamine moiety [211]. The initial effort in discovering SQ109 was undertaken using a combinatorial chemistry approach to find an EMB analog with improved activity because EMB is the weakest agent of the frontline therapeutics. However, retrospective analysis using SQ109-resistant mutants showed that this inhibitor's mode of action differs from EMB, with action associated with MmpL3 inhibition. It is now accepted that SQ109 has polypharmacology properties, as it has activity on fungi and bacteria that do not possess mycolic acids $[212,213]$ and activity against latent cells that do not require active cell wall synthesis [162, 214]. Further investigation of SQ109's mechanism of action divulged additional inhibition of menaquinone synthesis, cellular respiration, and ATP synthesis that results in a dissipation of the electrochemical proton gradient [162]. These multiple mechanisms of antitubercular activities suggest that SQ109 (Figure 2-18) would be an effective agent to add to MDR-TB therapies and will cause limited instances of resistance if used in such a regimen, though it does have some pharmacological limitations due to its amphipathic structure.

More specific classes of MmpL3 inhibitors were discovered via a whole-cell phenotypic screen performed at the Novartis Institute for Tropical Diseases (NITD; Singapore). Indolcarboxamides (Figure. 2-18) that have excellent PD properties, including potent bactericidal activity, display both concentration and time-dependent killing [215]. Lead molecules are orally bioavailable and display limited toxicity, including no inhibition of HepG2, hERG, AMES, or CYP450. They possess a narrow spectrum of activity against gram-positive and -negative bacteria, a positive attribute for a potential antitubercular agent. Researchers working with indolcarboxamide-resistant Mtb discovered no cross-resistance associated with any commonly used drugs for Mtb treatment. Cross-resistance was noted with other MmpL3 inhibitors, including SQ109 (16-fold shift in MIC), AU1235 (>1,024-fold, Figure 2-18), and BM212 (2-fold, Figure 2-18) [216]. This range of MIC shifts can be explained by different binding sites of the inhibitors in MmpL3 and that fact that some MmpL3 inhibitors are notorious polypharmacologic. One crucial advantage that the indolcarboxamides display is a dramatic accumulation in the lungs in vivo. Early analogs of the initial HTS hit showed a 5-fold higher $\mathrm{C}_{\max }$ and a 10-fold increase in $\mathrm{AUC}_{0-24 \mathrm{~h}}$, in respect to lung concentrations vs. plasma concentrations of drug [216]. In general, MmpL3 inhibitors are quite lipophilic, which is not surprising, given the role of MmpL3 in exporting mycolic acids. This lipophilic nature, in turn, causes problems with distribution and propensity for oxidative metabolism of the inhibitors. 


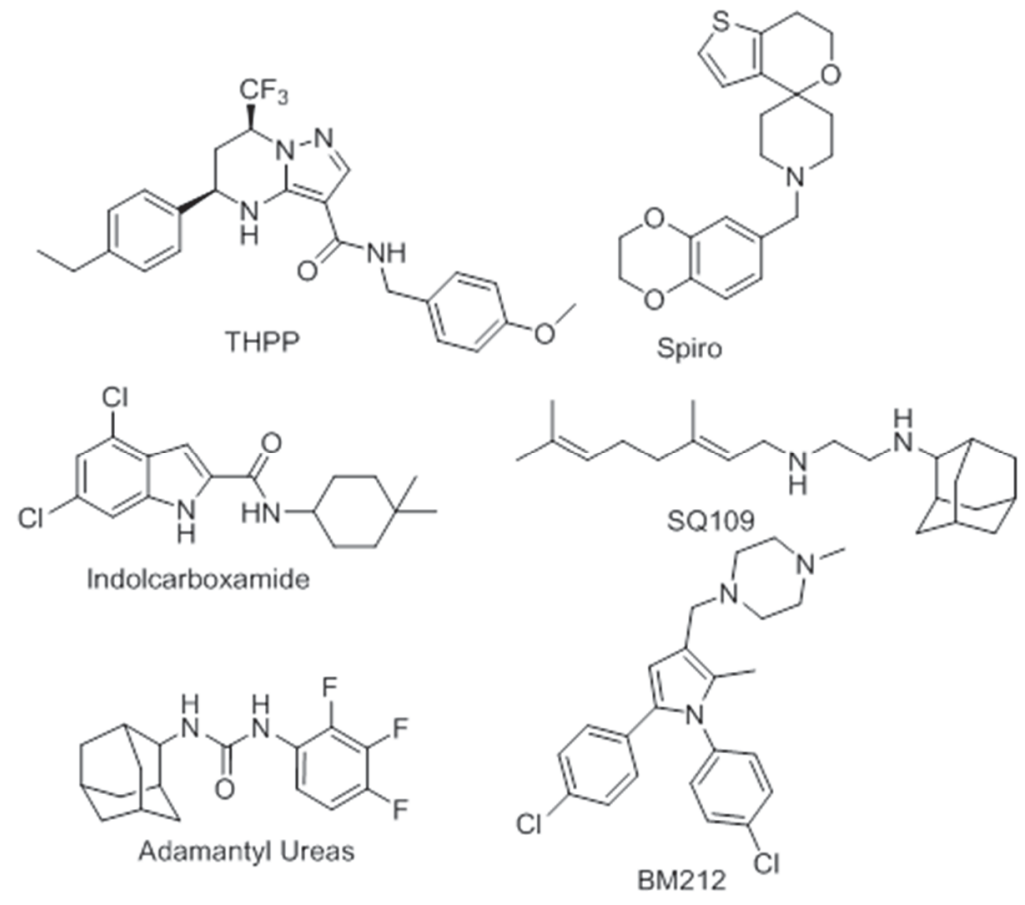

Figure 2-18. MmpL3 inhibitors

The class of MmpL3 inhibitors is highly chemically diverse, but is hallmarked by lipophilic molecules with less than ideal physiochemical properties. 


\section{Isocamphonyl Ureas for TB}

In the past we have identified multiple series of ureas with potent Mtb activity. These were initially discovered via a phenotypic high throughput screening (HTS) effort of 12,000 compounds and further optimized in multiple stages to improve physicochemical properties. Early iterations of this initial hit were noted to be very structurally similar to mammalian soluble epoxide hydrolase (SEH) inhibitors as well as Mtb epoxide hydrolase enzymbe B (EphB) inhibitors, which was the presumed target of the urea HTS hit [217, 218] (Figure 2-19). Early medicinal chemistry efforts not only attempted to improve on the poor in vitro adsorption, distribution, metabolism, elimination and toxicity (ADMET) properties but to gain selectivity away from the human isoform of SEH. It was only through genome sequencing of lead compound resistant mutants that the true target of these inhibitors was identified. It was identified there was a single point mutation in the gene that encodes the MmpL3 transport protein that transports trehalose monomycolates across the inner membrane of the Mtb cell envelope to the outermost layer of mycolic acids [219]. MmpL3 is the only member of the MmpL family that is essential for Mtb growth and survival and is a frequently identified target of phenotypic screens [210, 219]. The lead MmpL3 inhibitor SQ109 [220] is in Phase II clinical trials to treat TB and MDR-TB as a potential replacement for the currently used ethambutol, another mycolic acid disrupting agent. This work focuses on continuing earlier efforts to optimize already mycobacterium potent ureas previously identified from a HTS phenotypic screen $[217,221]$. These previous ureas were quite potent in vitro against Mtb but had very poor ADMET properties; notably poor solubility, high plasma protein binding and poor metabolic stability (1235, Figure 2-20). This series also suffered from an off target promiscuity issue, with relatively high inhibition of human soluble epoxide hydrolase $(\mathrm{hSEH})$. The driving force of this exploration was to determine the impact of a pharmacological biosteric replacement of the adamantyl ring with a unique lipophilic ring system, the iso-camphonyl ring. Iso-camphonyl is a structural derivative of the natural product camphor, a fused ring system with a host of pharmacological properties and very well tolerated in humans. In previous studies it was discovered that substituted heteroaromatics gave the greatest Mtb activity and modulation of these was used to tweak in vitro ADMET properties (Figure 2-20). This study looks to incorporate previous SAR studies on the adamantly urea with the pharmacological biostere iso-camphonyl moiety. Efforts are focused on improving PK properties such as solubility and microsomal stability while maintaining the robust Mtb activity and find an agent with in vivo efficacy that is still very safe.

\section{Results}

The goal for this series was to improve pharmacokinetic properties and increase selectivity for Mtb MIC versus sEH while maintaining or improving ADMET properties from previous generation of adamantly urea compounds. The compounds were synthesized by first forming an isocyanate from $1 \mathrm{R}, 2 \mathrm{R}, 3 \mathrm{R}, 5 \mathrm{~S}$ - isocamphonyl amine using triphosgene at $0^{\circ} \mathrm{C}$. This mixture was added carefully to substituted hetroaromatic amines deprotonated by butyl lithium at $-78^{\circ} \mathrm{C}$. Care had to be used in temperature 
<smiles>[Y16]S(=O)(=O)NC1C2CC3CC(C2)CC1C3</smiles>

hSEH IC50: $0.4 \mathrm{nM}$<smiles>O=C(Nc1ccccc1)Nc1ccccc1</smiles>

MtbSEH IC50: $19 \mathrm{nM}$

Figure 2-19. The initial HTS hit (1235) compared structurally to a known Mtb soluble epoxide hydrolase inhibitor.

Concerns of specificity spurned the initial medicinal chemistry investigations.<smiles>O=C(Nc1ccc(F)c(F)c1F)NC1C2CC3CC(C2)CC1C3</smiles>

1235

Mtb MIC: $0.01 \mathrm{ug} / \mathrm{mL}$

Solubility: $>0.1 \mathrm{ug} / \mathrm{mL}$

HPPB: $99 \%$<smiles>CC(C)(C)c1cc(NC(=O)NC2C3CC4CC(C3)CC2C4)no1</smiles>

1608

Mtb $\mathrm{MIC}=0.1 \mu \mathrm{g} / \mathrm{mL}$

Solubility $=22.7 \mu \mathrm{g} / \mathrm{mL}$

M. Stability $\mathrm{t} 1 / 2=1.38 \mathrm{hr}$

HPPB $=95.2 \%$

Figure 2-20. Initial optimization

After two reported iterations of medicianal chemistry optimizations, 1608 emerged as a lead compound with improved drug-like properties and comparable MIC activity. 
regulation and speed of addition to avoid the production of symmetric isocamphonyl ureas that were the main byproduct of these reactions. Compounds were then evaluated for whole cell Mtb activity before having their in vitro ADMET properties tested by the High Throughput Analytical Chemistry (HTAC) core (Table 2-3). The new isocamphonyl caged ring system we were able to improve on the MIC activity of the more potent compounds from previous series such as 1608 (Figure 2-21) and increase the LiPE. We were also achieved selectivity away from $\mathrm{hSEH}$ inhibiton, doubling the $\mathrm{IC}_{50}$ from 1608 while improving MIC activity which opens our therapeutic window wider. However, we were unable to dramatically improve or even maintain PK properties (Figure 2-21) decreasing in vitro solubility, metabolic stability and returning to $99 \%$ plasma protein binding. In the case of compound 1, a t-butyl isoxazole isocamphonyl urea, we were able to get a lead compound for our series that was submitted for in vivo studies. From this compound we will discuss some general SAR, mainly involving the hetero-aromatic off the 3 position of the urea core.

It tracked across most similar compounds that the more polarity an analog possessed (lower cLogP) the better PK parameters it would have. This increase in solubility and lower plasma protein binding generally came at a cost to Mtb activity as MIC values increased. We have applied the same LiPE efficiency coefficient to directly compare compounds in relation to their lipophilicity. Comparing $\mathbf{1}$ to $\mathbf{2}$ these is a clear shift in the MIC but a noticeable improvement in solubility, marginal improvement in protein binding and microsomal stability with a simple t-butyl to methyl swap on the same isoxazole ring. Overall there is a decrease in the LiPE from 3.30 to 0.33 (Figure 2-22). This trend is replicated across multiple aromatic groups such as when comparing 3 and 4 to 5 , removing a methyl from the thiazole ring to again decrease lipophilicity, with the same negative effects on MIC and slight improvements on PK properties. This is possibly due to the location and lipophilic substrates of the presumed cellular target MmpL3. This is a membrane bound protein that transports mycolic acids to the cell surface.

Next, the opposite enantiomer of 1, 2, 3, 6 and $\mathbf{8}$ were synthesized. Switching the chirality of the four chiral centers in the ring system results in a ring that is essentially turned inside out, which allows us to probe a different chemical space relative to the unchanged urea and substituted heterocycle. This was achieved by using the $1 \mathrm{~S}, 2 \mathrm{~S}, 3 \mathrm{~S}$, $5 \mathrm{R}$ enantiomer of the iso-camphonyl amine in the same reaction with triphosgene to generate the isocyanate and urea formation with a deprotonated heteroaromatic amine. This inversion was shown to be deleterious to Mtb activity, in addition to not providing any benefits to PK properties (Figure 2-23). This demonstrates that wherever that drug is binding, it is not solely dependent on lipophilicity but its site is spatially constrained; most likely through specific binding to a protein target rather than simple membrane disruption. If these compounds were simply integrating into the membrane and causing destabilization than an alteration to how the ring orients in space would not affect activity; but if there is a specific site in a protein that it binds to than altering the molecule in this way could disfavor binding causing a loss in efficacy. This same spatial restriction phenomenon was noted in the previous adamantly urea series, where changing the 
Table 2-3. All urea compounds with in vitro data

\begin{tabular}{|c|c|c|c|c|c|c|c|c|}
\hline$\#$ & Structure & $\begin{array}{r}\text { Mtb MIC } \\
(\mu \mathrm{g} / \mathrm{mL})^{1}\end{array}$ & $c \log \mathrm{P}^{2}$ & LiPE & $\begin{array}{l}\text { Solubility } \\
(\mu \mathrm{g} / \mathrm{mL})^{3}\end{array}$ & $\begin{array}{c}\text { Protein } \\
\text { Binding }(\%) \\
\end{array}$ & $\begin{array}{c}\text { Microsomal } \\
\text { Stability }^{4}\end{array}$ & $\begin{array}{c}\text { sEH IC } 50 \\
(n M)^{5}\end{array}$ \\
\hline 1 & & 0.049 & 5.484 & 3.23 & 7.8 & 99.1 & 0.90 & 132.6 \\
\hline 2 & & 3.13 & 4.157 & 0.33 & 14.8 & 98.1 & 0.93 & 139.1 \\
\hline 3 & & 0.098 & 4.855 & 3.15 & 3.8 & 99.3 & 0.40 & 397.1 \\
\hline 4 & & 0.391 & 4.855 & 1.77 & 6.0 & 99.6 & 0.80 & 178.5 \\
\hline 5 & & 3.13 & 4.356 & 0.14 & 15.8 & 98.9 & 0.86 & 220.5 \\
\hline 6 & & 0.049 & 5.980 & 2.76 & 0.2 & 98.8 & 0.70 & 4.2 \\
\hline 7 & & 0.02 & 5.950 & 3.76 & 0.12 & 77.3 & 14.18 & 298.7 \\
\hline 8 & & 3.13 & 4.146 & 0.48 & 29.9 & 98.5 & 1.13 & 452.8 \\
\hline 9 & & 3.13 & 4.071 & 0.51 & 13.2 & 98.6 & 0.71 & 1331.6 \\
\hline 10 & & 0.098 & 5.329 & 2.84 & 6.5 & 98.2 & 0.72 & 5.7 \\
\hline
\end{tabular}


Table 2-3. (continued)

\begin{tabular}{|c|c|c|c|c|c|c|c|c|}
\hline$\#$ & Structure & $\begin{array}{c}\text { Mtb MIC } \\
(\mu \mathrm{g} / \mathrm{mL})^{1}\end{array}$ & $\operatorname{cLog} P^{2}$ & LiPE & $\begin{array}{l}\text { Solubility } \\
(\mu \mathrm{g} / \mathrm{mL})^{3}\end{array}$ & $\begin{array}{c}\text { Protein } \\
\text { Binding }(\%)\end{array}$ & $\begin{array}{c}\text { Microsomal } \\
\text { Stability }^{4}\end{array}$ & $\begin{array}{c}\text { sEH IC } 50 \\
(n M)^{5}\end{array}$ \\
\hline 11 & & 0.39 & 4.883 & 1.82 & 6.2 & 99.4 & 1.52 & 357.1 \\
\hline 12 & & 12.5 & 4.584 & -1.38 & 2.7 & 97.5 & 0.31 & 463.7 \\
\hline 14 & & $>200$ & 4.157 & -3.83 & 21.0 & 97.7 & 0.14 & 322.6 \\
\hline 15 & & 12.5 & 4.855 & -1.70 & 5.8 & 99.0 & 0.17 & 1670.4 \\
\hline 16 & & 3.125 & 5.980 & -1.39 & 0.4 & 98.6 & 0.21 & 3.5 \\
\hline 17 & & 25 & 4.146 & -1.60 & 26.5 & 98.0 & 0.88 & 798.2 \\
\hline 18 & & $>200$ & 4.071 & -3.64 & 21.1 & 98.1 & 0.71 & 960.1 \\
\hline
\end{tabular}


Table 2-3: (continued)

\begin{tabular}{|c|c|c|c|c|c|c|c|c|}
\hline \# & Structure & $\begin{array}{l}\text { Mtb MIC } \\
(\mu \mathrm{g} / \mathrm{mL})^{1}\end{array}$ & $\operatorname{cLog} P^{2}$ & LiPE & $\begin{array}{l}\text { Solubility } \\
(\mu \mathrm{g} / \mathrm{mL})^{3}\end{array}$ & $\begin{array}{c}\text { Protein } \\
\text { Binding }(\%)\end{array}$ & $\begin{array}{c}\text { Microsomal } \\
\text { Stability }^{4}\end{array}$ & $\begin{array}{c}\text { sEH IC } 50 \\
(n M)^{5}\end{array}$ \\
\hline 19 & & 12.5 & 4.670 & -1.39 & 8.3 & 99.2 & 0.21 & 413.5 \\
\hline 20 & & 50 & 4.920 & -3.02 & 12.5 & 99.2 & 0.11 & 1791.5 \\
\hline
\end{tabular}

\section{Notes}

${ }^{1}$ In vitro Mtb MIC using H37RV ${ }^{2}$ Calculated log of partition coeffiecent between octanol and water ${ }^{3}$ Solubility in simulated biological fluid ${ }^{4}$ Chemical stability in purified human hepatocytes in hours ${ }^{5}$ In vitro inhibition of human soluble epoxide hydrolase 


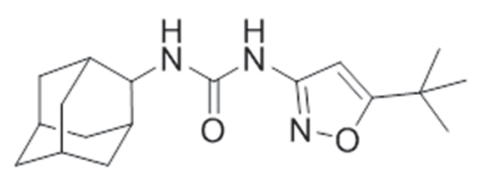

1608

$\mathrm{MIC}=0.1 \mu \mathrm{g} / \mathrm{mL}$

Solubility $=22.7 \mu \mathrm{g} / \mathrm{mL}$

M. Stability $t_{1 / 2}=1.38 \mathrm{hr}$

HPPB $=95.2 \%$

$\mathrm{hSEH}$ IC $\mathrm{C}_{50}=73 \mathrm{nM}$

$\mathrm{LiPE}=2.74$

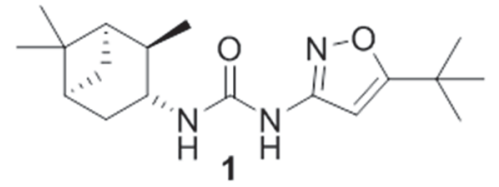

$\mathrm{MIC}=0.049 \mu \mathrm{g} / \mathrm{mL}$

Solubility $=7.8 \mu \mathrm{g} / \mathrm{mL}$

M. Stability $\mathrm{t}_{1 / 2}=0.90$

HPPB $=99.1 \%$

hSEH IC ${ }_{50}=132.6 \mathrm{nM}$

$\mathrm{LiPE}=3.30$

Figure 2-21. Urea lead comparisons

Comparison of the physiochemical properties of the previous 2-adamantyl urea lead 1608 with the iso-camphonyl lead $\mathbf{1}$. There was an improvement in lipophilic efficiency that may be able to overcome the decrease in some PK properties. 

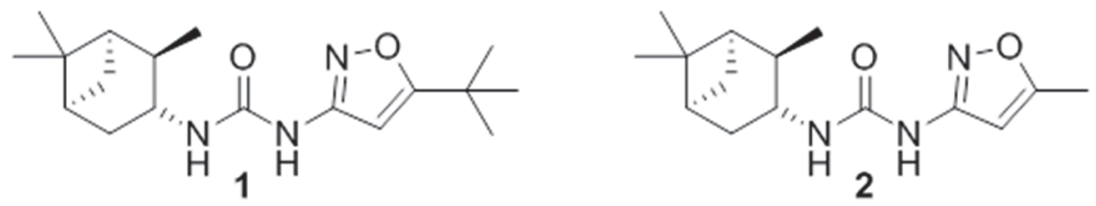

$\operatorname{cLog} P=5.485$

cLogP $=4.157$

Mtb MIC $=0.049 \mu \mathrm{g} / \mathrm{mL}$

Solubility $=7.8 \mu \mathrm{g} / \mathrm{mL}$

Mtb MIC $=3.13 \mu \mathrm{g} / \mathrm{mL}$

Solubility $=14.8 \mu \mathrm{g} / \mathrm{mL}$

$\mathrm{LiPE}=3.30$

LePE $=0.33$

Figure 2-22. Decreasing lipophilicity

Replacing a t-butyl with a methyl resulted in a dramatic reduction in potency and a tenfold hit to lipophilic efficiency.
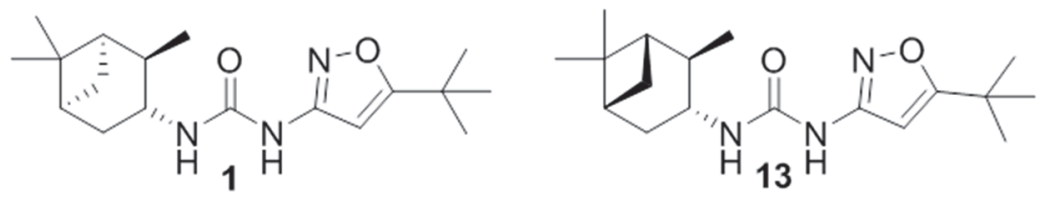

CLogP $=5.485$

Mtb MIC $=0.049 \mu \mathrm{g} / \mathrm{mL}$

CLogP $=5.484$

Mtb MIC $=6.25 \mu \mathrm{g} / \mathrm{mL}$

Solubiility $=7.8 \mu \mathrm{g} / \mathrm{mL}$

Solubility $=8.7 \mu \mathrm{g} / \mathrm{mL}$

$\mathrm{LiPE}=3.30$

LiPE $=-1.55$

Figure 2-23. Inversion of the stereochemistry

On the iso-camphonyl ring resulted in a 100fold increase in Mtb MIC activity. This implies there is a spactial limitation to the molecule and that it is binding to a specific site rather than simple membrane disruption. 
attachment point of the amine from the 1 to 2 position of the ring system resulted in a 10 fold increase in Mtb activity [221]. With roughly 100 fold reduction in activity, the generation of further analogs with this enantiomer was quickly abandoned.

Finally a brief study was conducted to see if the urea core could be replaced by a 2-aminoactamide to achieve free secondary amines, similar to other proven TB drugs like SQ109. The theory behind this is the free amine could aid in a greater volume of distribution of the drug and a higher degree of lung accumulation, which would make these more potent in vivo drugs [222]. Using a slightly different synthesis, chloroacetylchloride was coupled with either the lipophilic ring system or the t-butyl isoxazole amine first and the other second to generate 19 and 20 (Figure 2-24). However, it was easily seen with both the iso-camphonyl and previously studied adamantyl that the efficacy was lost when a methylene spacer was placed on either side of the urea to give a secondary amine proximal to either ring. The modest gains in the PK profile were not enough to overcome the dramatic loss in efficacy so this series was also discontinued.

After the brief medicinal chemistry exploration around the urea core, compound 1 was selected for in vivo studies. First the maximum tolerated dose was evaluated to see how safe this compound was. 1 dose per day (QD) was administered into C57BL/6 mice from Charles River Laboratories (females, 6-8 weeks old) for 3 days, followed by 7 more days of observation. Compound 1 was found to be safe at 100, 200 and $400 \mathrm{mg} / \mathrm{kg}$ given both subcutaneously and by oral gavage. This safety profile was encouraging and pushed for further PK trials for $\mathbf{1}$ to establish proper dosing regimens for an efficacy trial. We were able to improve oral bioavailability over the adamantyl lead compound from $\mathrm{F}=$ $2.0 \%$ to $\mathrm{F}=8.6 \%$ but still not in the range to be therapeutically relevant for oral administration (Table 2-4). Using a two-compartment model for PK analysis we found after an IV dose of $10 \mathrm{mg} / \mathrm{kg}$ that plasma levels of 1 reached $4.27 \mathrm{mg} / \mathrm{L}$ and had an average area under the curve (AUC) of $5.04 \mathrm{mg}^{*} \mathrm{hr} / \mathrm{L}$. The average half-life was 6.98 hours and the compound achieved a modest volume of distribution of $5.01 \mathrm{~L} / \mathrm{kg}$.

\section{Discussion}

These data suggest that $\mathbf{1}$ could be efficacious in vivo if oral bioavailability could be improved, or if the route of administration was altered. Recently bedaquiline has been approved as an oral treatment for MDR-TB, despite being highly lipophilic and violating many Lipinski's rules for oral bioavailability [55, 223]. In addition, newer therapeutic options of directly delivering agents to pulmonary lesions via aerosol are gaining traction and could be a more effective way to deliver our potent compounds [224]. Since these are very well tolerated compounds in regards to any acute toxicity, these exploratory PK formulation studies could be very helpful in producing ideal drug compounds for clinical success. Currently there are no MmpL3 inhibitors in the clinic, so this series fits most of the criteria for an emerging lead candidate in MDR-TB; highly potent for the possibility of reducing therapy time, no cross resistance with current agents and cheap to produce with facile chemistry. However there are limitations to formulation that would make once-a-day oral dosing almost impossible. It should be noted that this series was by and 


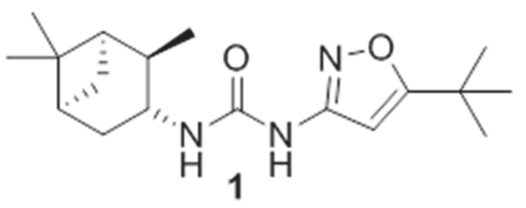

$\operatorname{cLog} P=5.485$

Mtb MIC $=0.049 \mu \mathrm{g} / \mathrm{mL}$

Solubility $=7.8 \mu \mathrm{g} / \mathrm{mL}$

$\mathrm{LiPE}=3.30$

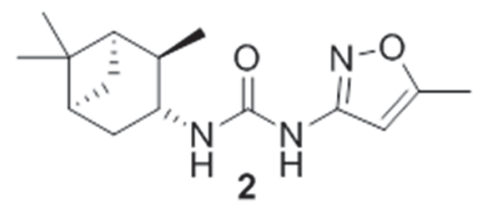

$\operatorname{cLog} \mathrm{P}=4.157$

Mtb MIC $=3.13 \mu \mathrm{g} / \mathrm{mL}$

Solubility $=14.8 \mu \mathrm{g} / \mathrm{mL}$ LePE $=0.33$

Figure 2-24. Decreasing lipophilicity

Replacing a t-butyl with a methyl resulted in a dramatic reduction in potency and a tenfold hit to lipophilic efficiency.

Table 2-4. Compound 1 in vivo PK data

\begin{tabular}{cccccc}
\hline Rat & $\begin{array}{c}\text { AUCpo } \\
\text { (hr* mg/L) }\end{array}$ & AUCiv & Dose $_{\text {po }}(\boldsymbol{\mu g})$ & Doseiv $(\boldsymbol{\mu g})$ & $\mathbf{\% F}$ \\
\hline 1 & 3.75 & 4.71 & 24100 & 2220 & 7.33 \\
2 & 5.47 & 5.36 & 24400 & 2220 & 9.27 \\
3 & 4.26 & 5.05 & 23900 & 2230 & 7.87 \\
4 & 5.24 & 5.02 & 23200 & 2220 & 10.0 \\
Mean: & 4.67 & 5.04 & 23900 & 2222.5 & 8.62 \\
\hline
\end{tabular}


large mycobacterial specific, having limited MIC activity in a panel of 15 gram-positive and negative species. This reassures that they are on target since prokaryotes do not possess mycolic acids and thus do not need their transport for effective cell replication.

\section{Discussion on TB Drug Discovery}

All of our efforts to develop new drugs to treat drug resistant Mtb have always been designed with the same principles core to the field as a whole. A series must have anovel mechanism of action, whether that is a new enzymatic target, a new biosynthetic pathway or a unique means of hitting a clinically relevant area that eludes its common resistance mechanisms. There is very little point in developing agents that might well be cross resistant to currently used therapies, especially in a disease area like tuberculosis where the resources and available medications are already scare. Next, a lead molecule should possess good drug-like properties such as solubility and plasma stability, in the hopes of easy formulation for once a day oral therapy. We have applied a host of drug discovery techniques to try and fill the discovery pipeline with good molecules for further development. We have described exploring an old clinical target in new ways in trying to find novel FAS-II inhibitors. This approach has allowed us to harness many target based discovery techniques to assess enzymatic activity and biophysical interactions with the protein of interest, as well as screen known Mtb active compounds to identify existing material that works at this target. I also detailed work on a phenotypic screening hit that required further optimization despite robust $\mathrm{Mtb}$ activity from the start.

Our Enoyl-ACP dehydratase inhibitors offer a unique way of inhibiting a clinically relevant drug target. We have shown ways to re-explore a validated enzymatic protein in HadAB that is the target of ISO and TAC in a manner that overcomes their two most common resistance mechanisms as well as eliminates the thiourea moiety that leads to poor PK properties and some toxicities. With our unique mechanism of semi covalent, reversible inhibitors we are also seeking to harness the long residency afforded by covalent inhibitors while circumventing off target binding that may have limited their dosage and duration of use. All of this combined with a fragment based screen that has identified efficient binders that are primed for structure guided design led by efficiency metrics to ensure drug-like properties. This project has a long way to go before generating clinical compounds of interest, but has strong possibilities along the way to make useful compounds. Producing and confirming a reversible covalent inhibitor library will greatly aid in studying the underlying mechanisms of the dehydratase enzyme, specifically the crosslinking inactivation. Tight binding fragments can be grown to strong inhibitors, some for attachment of the acrylonitrile but others can be grown out for better biochemistry control compounds. For example, tight binders can have fluorescent probes attached for fluorescence polarization assays which would be a vastly superior biochemical screening assay than what we currently have.

Phenotypic screens are not always significantly better at generating drug-like hits. Phenotypic screens tend to produce large, lipophilic molecules with a high degree of aromaticity that lead to poorly soluble drugs, for reasons to be discussed in Chapter four. 
This, combined with the fact that medicinal chemistry optimizations only tend to add grease to molecules (or at least do so at a higher rate than adding polarity) quickly exacerbates issues in solubility, plasma stability (high hepatic metabolism), CYP issues and cardiac toxicities. Our urea series for Mtb highlights these short comings. The series presented is the third round of optimizations on a phenotypic screening hit, and still fall short in terms of obtaining a true drug-like lead. It is true that there have been significant advances in formulations that make highly potent "bad" drugs efficacious (i.e. bedaquiline), but even this successful example is stuck with cardiac liabilities and an inability to penetrate all of Mtb's life cycles. Efficiency metrics are almost a must for phenotypic screens, and I believe curated libraries with chemical properties in mind are the next step in advancing phenotypic screening. In our efforts to optimize a phenotypic screening hit I have learned a great deal about the importance of drug-like properties and how difficult it can be to modulate them. We describe many attempts to alter our active scaffold in the hopes of making a better behaved drug; none of which were very

successful. Despite having a highly potent series we could not get the required PK to have an effect in vivo, something the field struggles with quite a bit. The transition from in vitro potency to in vivo efficacy is the most challenging aspect in drug discovery, and it is a transition that is made more difficult in Mtb. Efficiency metrics are just as important in phenotypic screening as they are in target based, and tracking a series progress with such markers early will only improve their long term chances.

\section{Materials and Methods}

\section{FAS-II Methods}

\section{Protein purification}

HadAB fused protein was expressed in BL21* (DE) cells. These cells were cultured in the presence of ampicillin and colonies that grew were picked stock cultures were established and frozen. Stock culture was grown in 4L of ampicillin containing growth media plus $1 \%$ glucose at $37^{\circ} \mathrm{C}$. At mid log $1 \mathrm{mM}$ IPTG was added and cells were incubated 16 hours at $18^{\circ} \mathrm{C}$. Cells were then centrifuged at $25000 \mathrm{rpm}$ for 30 minutes. Pellets were re-suspended in lysate buffer $(50 \mathrm{mM}$ Tris- $\mathrm{HCl} \mathrm{pH} 8.0,150 \mathrm{NaCl}, 10 \%$ glycerol and Roche protease inhibitor cocktail) before sonication for lysis. Cell debris and unburst cells were spun down again and supernatant was purified using GE His-Trap affinity and size exclusion columns to produce pure HadAB. Verified using LC-MS using an Agilent 6220 TOF (time-of-flight) mass spectrometer equipped with a multimode source (positive mode) and an Agilent 1200 binary pump. The column used was a Zorbax $(2.1 \times 150 \mathrm{~mm} 300 \mathrm{SB}-\mathrm{C} 83.5 \mu \mathrm{M})$ and separation was done using a gradient of solvent $\mathrm{A}$ (water, $1 \%$ formic acid) and solvent $\mathrm{B}$ (acetonitrile, $1 \%$ formic acid). The drying gas temperature was $310{ }^{\circ} \mathrm{C}$, and the vaporizer temperature was set at $200{ }^{\circ} \mathrm{C}$. Flow rate was $0.25 \mathrm{ml} / \mathrm{min}$, and the total run time was $27 \mathrm{~min}$. The fragmentor voltage was set to $180 \mathrm{~V}$. The runs were later analyzed using the Agilent's Mass Hunter program. Protein was concentrated to $5 \mu \mathrm{g} / \mathrm{mL}$ stock solutions and flash frozen before storage at $-80^{\circ} \mathrm{C}$. 


\section{Spectrophotometric enzymatic assay}

Hydratase activity was monitored by spectrophotometry at $263 \mathrm{~nm}$ in the presence of trans-2-dodecenoyl-CoA (C12:1-CoA) substrate $(500 \mu \mathrm{M})$ using a spectrophotometer. Comparison of the initial reaction rates obtained for the trans-2-enoyl-CoAs (C4 to C20) was performed at a fixed substrate concentration and $80 \mathrm{nM}$ HadAB plus $100 \mu \mathrm{M}$ of the inhibitors. Control experiments lacking the enzyme were included in each run.

\section{NMR analysis of HadAB reaction}

Trans-2-dodecenoyl-CoA (C12:1-CoA) substrate $(500 \mu \mathrm{M})$ was dissolved into buffer $\left(50 \mathrm{mM} \mathrm{PO}_{4}, 2 \mathrm{mM}\right.$ dueterated-DTT and $150 \mathrm{mM} \mathrm{NaCl}$ in $\left.\mathrm{D}_{2} \mathrm{O}\right)$ and protein $(200$ $\mathrm{nM}$ ) was added to a final volume of $600 \mu \mathrm{L}$. Solution was scanned using water suppression via excitation sculpting ${ }^{1} \mathrm{H}$ method on a $500 \mathrm{MHz}$ Bruker NMR. Time points were taken at initial mixing, 2 minutes, 5 minutes, 10 minutes, 20 minutes and 45 minutes. Spectra were then analyzed using a reaction monitoring method from the MestReNova suite.

\section{NMR analysis of 3216 inhibition of $\mathrm{Had} A B$ reaction}

Protein $(200 \mathrm{nM})$ and inhibitor $(100 \mu \mathrm{M})$ were added to dueterated buffer and allowed to incubate for 5 minutes. Substrate was added to a final volume of $600 \mu \mathrm{L}$ and spectra were collected and analyzed using the previously described methods.

\section{Fragment screening by SPR}

Fragment library (4,500 compounds) was procured from St. Jude inventory and composed of commercially available fragment libraries from Life, Enamine and Maybridge. Fragments have previously been curated to remove indiscriminate protein destabilizers and those which interfered with many assays. SPR experiments were conducted at $20^{\circ} \mathrm{C}$ using a SensiQ Pioneer optical biosensor (SensiQ Technologies). Neutravidin (Thermo Sci-entific) was covalently immobilized on a polysaccharide hydrogel-coated gold surface (COOH5 chip; SensiQ Technologies) using routine amine coupling chemistry in immobilization buffer (10 mM HEPES pH 7.4, $150 \mathrm{mM} \mathrm{NaCl}$, $0.005 \%$ Tween 20$)$. Carboxyl groups on the hydrogel were activated with $\mathrm{N}$-ethyl- $\mathrm{N}^{0}-(3-$ dimethylamino-propyl) carbodiimide (EDC) and N-hydroxysuccinimide (NHS), and neutravidin was injected until immobilization levels of 5000- 7000 RU were achieved. Remaining active sites were blocked by reaction with ethanolamine. The instrument was primed with binding buffer (20 mM Tris $\mathrm{pH} 8.5,100 \mathrm{mM} \mathrm{NaCl}, 10 \mathrm{mM} \mathrm{MgCl}, 1 \mathrm{mM}$ TCEP, $0.005 \%$ Tween 20, 10\% glycerol, 5\% DMSO), and HadAB was injected until 3000-3700 RU of protein was captured. Unoccupied biotin-binding sites of neutravidin on both the reference and HadAB surfaces were blocked with amine- $\mathrm{PEG}_{2}$-biotin (Thermo Scientific) to minimize potential non-specific binding by the compounds. ${ }^{35}$ The compounds were prepared in running buffer as a 3-fold dilution series starting at $100 \mu \mathrm{M}$ and were injected at a flow rate of $80 \mu \mathrm{L} / \mathrm{min}$. A series of buffer-only (blank) injections was included throughout the experiment to account for instrumental noise. The data were processed, double-referenced, solvent cor-rected and analyzed ${ }^{35,36}$ using the software 
package Qdat (version 2.1.0.21, BioLogic Software). The equilibrium dissociation constants were determined by fitting the data to a 1:1 interaction model

\section{LC-MS-MS}

Chromatographic separations were carried out using a Shimadzu liquid chromatograph (Shimadzu Corporation, USA) consisting of two pumps, online degasser, system controller and a CTC Leap auto sampler (Leap Technologies, Carrboro, NC). Mobile phase consist of $0.1 \%$ Formic acid spiked acetonitrile (Pump B) and $0.1 \%$ Formic acid spiked HPLC grade water (Pump A) was used at a flow rate of $0.6 \mathrm{~mL} / \mathrm{min}$ in gradient mode. A Phenomenex ${ }^{\circledR} \mathrm{C} 18(2), 3 \mu \mathrm{m}, 50 \times 2.0 \mathrm{~mm}$ column (Phenomenex, Torrance, CA) protected with a guard column was used for the separation. The samples $(20 \mu \mathrm{L})$ were injected on column and the eluate was led directly into a mass spectrometer.

\section{UPLC/MS/UV}

LC-MS chromasolv grade acetonitrile $(\mathrm{ACN})$ was purchased from Fisher Scientific (Loughborough, UK). LC-MS chromasolv grade methanol, ammonium bicarbonate and formic acid were obtained from Sigma-Aldrich (St. Louis, MO). Milli-Q water as an ultrapure laboratory grade water was used in aqueous mobile phase. Chromatographic separation was performed on an Acquity UPLC BEH C18 1.7 $\mu \mathrm{m}, 2.1$ x $50 \mathrm{~mm}$ column (Waters Corporation, Milford, MA) using an Acquity ultra performance liquid chromatography system. Data were acquired using Masslynx v. 4.1 and analyzed using the Quanlynx software suite. This was coupled to an SQ mass spectrometer. The total flow rate was $1.0 \mathrm{~mL} / \mathrm{min}$. The sample injection volume was $10 \mu \mathrm{L}$. The UPLC column was maintained at $55^{\circ} \mathrm{C}$. Samples were separated on the column under acidic or basic mobile phase based on their physical-chemical properties. For the acidic mobile phase, solvent A was $0.1 \%$ formic acid in MilliQ $\mathrm{H} 2 \mathrm{O}$ and solvent B was $0.1 \%$ formic acid in ACN. For the basic mobile phase, solvent A was $10 \mathrm{mM}$ ammonium bicarbonate in MilliQ H2O and solvent B was methanol. Samples were eluted from the column under one of the gradients listed in table 1. The mass spectrometer was operated in positive-ion mode with electrospray ionization. The conditions were as follows: capillary voltage 3.4 $\mathrm{kV}$, cone voltage $30 \mathrm{~V}$, source temperature $130{ }^{\circ} \mathrm{C}$, desolvation temperature $400{ }^{\circ} \mathrm{C}$, desolvation gas $800 \mathrm{~L} / \mathrm{hr}$, cone gas $100 \mathrm{~L} / \mathrm{hr}$. A full scan range from $\mathrm{m} / \mathrm{z}=110-1000$ in $0.2 \mathrm{~s}$ was used to acquire MS data. A single ion recording mass spectrometry for each compound was used to determine the quantification of the samples.

\section{General synthesis of aldehydes}

A microwave vial was charged with a stir bar and 4-hydoxybenzaldehyde was added $(1.221 \mathrm{~g}, 10 \mathrm{mmol})$ followed by alkylhalide (1.1 eq, $11 \mathrm{mmol})$, potassium carbonate $(2.073 \mathrm{~g}, 15 \mathrm{mmol})$ and DMF $(2 \mathrm{~mL})$. Vessel was sealed and heated to $120^{\circ} \mathrm{C}$ for 15 minutes. Water was added to reaction mixture and extracted with ethyl acetate. Organic layer was washed in sequence with $1 \mathrm{~N}$ sodium hydroxide, water and saturated sodium choloride before being dried over sodium sulfate and concentrated under reduced pressure. Crude was carried forward to subsequent steps without purification. 


\section{General synthesis of acrylonitriles}

Aldehyde (1eq, 0.2mmol), 2-nitrile (1.2eq, 0.24mmol) and triethyl amine were suspended in ethanol and added to a microwave vessel. Reaction heated to $70^{\circ} \mathrm{C}$ for 30 minutes, cooled to ambient temperature and concentrated. Crude residue was purified by flash chromatography using a 40-100\% hexane:ethyl acetate gradient.

Below is a listing of analytical purity of compounds discussed in this section. Experimental details for determination are described in the methods above.

(E)-2-((4-chlorophenyl)sulfonyl)-3-(4-(isopentyloxy)phenyl)acrylonitrile (3187) $56.9 \mathrm{mg}\left(73 \%\right.$ yield) white powder ${ }^{1} \mathrm{H}$ NMR $(500 \mathrm{MHz}$, Chloroform- $d) \delta 8.13(\mathrm{~s}, 1 \mathrm{H})$, 7.94 (dd, $J=14.6,7.9 \mathrm{~Hz}, 4 \mathrm{H}), 7.58$ (d, $J=7.9 \mathrm{~Hz}, 2 \mathrm{H}), 6.99$ (d, $J=8.2 \mathrm{~Hz}, 2 \mathrm{H}), 4.09$ (t, $J=6.6 \mathrm{~Hz}, 2 \mathrm{H}), 1.85(\mathrm{dp}, J=13.2,6.6 \mathrm{~Hz}, 1 \mathrm{H}), 1.72(\mathrm{q}, J=6.6 \mathrm{~Hz}, 2 \mathrm{H}), 0.99$ (d, $J=6.6$ Hz, 6H). ESI-MS [M+H]: Calc: 389.89 Found: 390.03

\section{(E)-3-(4-(isopentyloxy)phenyl)-2-(phenylsulfonyl)acrylonitrile (3188)}

$58.6 \mathrm{mg}\left(82 \%\right.$ yield) white powder ${ }^{1} \mathrm{H}$ NMR $(500 \mathrm{MHz}$, Chloroform- $d$ ) $\delta 8.05(\mathrm{~s}, 1 \mathrm{H})$, $7.93(\mathrm{~d}, J=8.1 \mathrm{~Hz}, 2 \mathrm{H}), 7.83(\mathrm{~d}, J=8.8 \mathrm{~Hz}, 2 \mathrm{H}), 7.60(\mathrm{t}, J=7.1 \mathrm{~Hz}, 1 \mathrm{H}), 7.51(\mathrm{t}, J=7.7$ $\mathrm{Hz}, 2 \mathrm{H}), 6.89(\mathrm{~d}, J=8.8 \mathrm{~Hz}, 2 \mathrm{H}), 1.75(\mathrm{dp}, J=13.3,6.7 \mathrm{~Hz}, 1 \mathrm{H}), 1.62(\mathrm{t}, J=6.7 \mathrm{~Hz}$, 2H), $0.88(\mathrm{~d}, J=6.7 \mathrm{~Hz}, 6 \mathrm{H})$. ). ESI-MS [M+H]: Calc: 355.45 Found: 356.13

(E)-3-(4-(isopentyloxy)phenyl)-2-tosylacrylonitrile (3189)

$42.5 \mathrm{mg}\left(57.5 \%\right.$ yield) off-white powder ${ }^{1} \mathrm{H}$ NMR $(500 \mathrm{MHz}$, Chloroform- $d$ ) $\delta 8.13$ (s, $1 \mathrm{H}), 7.91(\mathrm{t}, J=8.4 \mathrm{~Hz}, 4 \mathrm{H}), 7.40(\mathrm{~d}, J=8.0 \mathrm{~Hz}, 2 \mathrm{H}), 6.98(\mathrm{~d}, J=8.7 \mathrm{~Hz}, 2 \mathrm{H}), 4.09$ (t, $J$ $=6.6 \mathrm{~Hz}, 2 \mathrm{H}), 2.48(\mathrm{~s}, 3 \mathrm{H}), 1.85(\mathrm{dp}, J=13.3,6.7 \mathrm{~Hz}, 1 \mathrm{H}), 1.72(\mathrm{q}, J=6.7 \mathrm{~Hz}, 2 \mathrm{H}), 0.99$ $(\mathrm{d}, J=6.6 \mathrm{~Hz}, 6 \mathrm{H})$. ESI-MS [M+H]: Calc: 369.48 Found: 369.96

(E)-2-(4-chlorobenzoyl)-3-(4-(isopentyloxy)phenyl)acrylonitrile (3190)

$53.0 \mathrm{mg}$ (74.9\% yield) yellow powder ${ }^{1} \mathrm{H}$ NMR $(500 \mathrm{MHz}$, Chloroform- $d) \delta 8.06(\mathrm{~d}, J=$ $7.4 \mathrm{~Hz}, 3 \mathrm{H}), 7.86(\mathrm{~d}, J=7.5 \mathrm{~Hz}, 2 \mathrm{H}), 7.51(\mathrm{~d}, J=7.6 \mathrm{~Hz}, 2 \mathrm{H}), 7.02(\mathrm{~d}, J=8.2 \mathrm{~Hz}, 2 \mathrm{H})$, $4.12(\mathrm{t}, J=6.6 \mathrm{~Hz}, 2 \mathrm{H}), 1.87(\mathrm{dp}, J=13.2,6.6 \mathrm{~Hz}, 1 \mathrm{H}), 1.74(\mathrm{q}, J=6.5 \mathrm{~Hz}, 2 \mathrm{H}), 1.00$ (d, $J=6.6 \mathrm{~Hz}, 6 \mathrm{H})$. ESI-MS [M+H]: Calc: 353.85 Found: 354.05

(E)-3-(4-(2-morpholinoethoxy)phenyl)-2-(phenylsulfonyl)acrylonitrile (3209)

$103.8 \mathrm{mg}$ (97\% yield) dark red oil ${ }^{1} \mathrm{H}$ NMR $(500 \mathrm{MHz}$, Chloroform- $d$ ) $\delta 8.16(\mathrm{~s}, 1 \mathrm{H}), 8.03$ $(\mathrm{d}, J=7.9 \mathrm{~Hz}, 2 \mathrm{H}), 7.94(\mathrm{~d}, J=8.5 \mathrm{~Hz}, 2 \mathrm{H}), 7.72(\mathrm{t}, J=7.4 \mathrm{~Hz}, 1 \mathrm{H}), 7.63(\mathrm{t}, J=7.6 \mathrm{~Hz}$, 2H), $7.01(\mathrm{~d}, J=8.5 \mathrm{~Hz}, 2 \mathrm{H}), 4.27(\mathrm{~s}, 2 \mathrm{H}), 3.80(\mathrm{~s}, 4 \mathrm{H}), 2.92(\mathrm{~s}, 2 \mathrm{H}), 2.67$ (s, 4H). ESIMS [M+H]: Calc: 398.48 Found: 398.89

(E)-3-(4-(2-morpholinoethoxy)phenyl)-2-tosylacrylonitrile (3210)

$78.5 \mathrm{mg}\left(87 \%\right.$ yield) dark red oil ${ }^{1} \mathrm{H}$ NMR $(500 \mathrm{MHz}$, Chloroform- $d) \delta 8.11(\mathrm{~s}, 1 \mathrm{H}), 7.95$ $-7.81(\mathrm{~m}, 4 \mathrm{H}), 7.38(\mathrm{~d}, J=7.8 \mathrm{~Hz}, 2 \mathrm{H}), 6.98(\mathrm{~d}, J=7.7 \mathrm{~Hz}, 2 \mathrm{H}), 5.30$ (s, 3H), 4.19 (s, 2H), $3.80-3.65(\mathrm{~m}, 4 \mathrm{H}), 2.83$ (s, 2H), 2.58 (s, 4H). ESI-MS [M+H]: Calc: 412.50

Found: 413.09 
(E)-2-((4-chlorophenyl)sulfonyl)-3-(4-(2-morpholinoethoxy)phenyl)acrylonitrile (3211) $82.2 \mathrm{mg}$ ( $88 \%$ yield) orange powder ${ }^{1} \mathrm{H}$ NMR $(500 \mathrm{MHz}$, Chloroform- $d$ ) $\delta 8.12(\mathrm{~s}, 1 \mathrm{H})$, $7.92(\mathrm{t}, J=9.1 \mathrm{~Hz}, 4 \mathrm{H}), 7.57(\mathrm{~d}, J=9.8 \mathrm{~Hz}, 2 \mathrm{H}), 7.00(\mathrm{~d}, J=8.3 \mathrm{~Hz}, 2 \mathrm{H}), 4.20(\mathrm{~s}, 2 \mathrm{H})$, $3.73(\mathrm{~s}, 4 \mathrm{H}), 2.84$ (s, 2H), 2.59 (s, 4H). ESI-MS [M+H]: Calc: 432.92 Found: 433.07

(E)-3-(4-(2-morpholinoethoxy)phenyl)-2-(thiophene-2-carbonyl)acrylonitrile (3213) $40.0 \mathrm{mg}\left(54.3 \%\right.$ yield) orange powder ${ }^{1} \mathrm{H}$ NMR $(500 \mathrm{MHz}$, Chloroform- $d$ ) $\delta 8.28$ (d, $J=$ $3.8 \mathrm{~Hz}, 1 \mathrm{H}), 8.26(\mathrm{~s}, 1 \mathrm{H}), 8.09$ (d, $J=8.6 \mathrm{~Hz}, 2 \mathrm{H}), 7.78(\mathrm{~d}, J=4.9 \mathrm{~Hz}, 1 \mathrm{H}), 7.22(\mathrm{t}, J=$ $4.4 \mathrm{~Hz}, 2 \mathrm{H}), 7.04$ (d, $J=8.3 \mathrm{~Hz}, 2 \mathrm{H}), 4.28-4.23(\mathrm{~m}, 2 \mathrm{H}), 3.78(\mathrm{~s}, 4 \mathrm{H}), 2.91-2.87$ (m, 2H), 2.65 (s, 4H). ESI-MS [M+H]: Calc: 368.45 Found: 369.05

(E)-3-(4-(isopentyloxy)phenyl)-2-(thiophene-2-carbonyl)acrylonitrile (3214) $20.4 \mathrm{mg}\left(31.3 \%\right.$ yield) yellow powder ${ }^{1} \mathrm{H}$ NMR $(500 \mathrm{MHz}$, Chloroform- $d$ ) $\delta 8.30(\mathrm{~d}, J=$ $3.8 \mathrm{~Hz}, 1 \mathrm{H}), 8.28(\mathrm{~s}, 1 \mathrm{H}), 8.10(\mathrm{~d}, J=8.6 \mathrm{~Hz}, 2 \mathrm{H}), 7.78(\mathrm{~d}, J=4.9 \mathrm{~Hz}, 1 \mathrm{H}), 7.23(\mathrm{t}, J=$ $4.4 \mathrm{~Hz}, 1 \mathrm{H}), 7.03(\mathrm{~d}, J=8.6 \mathrm{~Hz}, 2 \mathrm{H}), 4.12(\mathrm{t}, J=6.6 \mathrm{~Hz}, 2 \mathrm{H}), 1.88(\mathrm{dt}, J=13.2,6.6 \mathrm{~Hz}$, $1 \mathrm{H}), 1.77-1.73(\mathrm{~m}, 2 \mathrm{H}), 1.01(\mathrm{~d}, J=6.7 \mathrm{~Hz}, 6 \mathrm{H})$. ESI-MS [M+H]: Calc: 325.43 Found: 326.11

(E)-2-(furan-2-carbonyl)-3-(4-(2-morpholinoethoxy)phenyl)acrylonitrile (3216) $46.5 \mathrm{mg}\left(66.0 \%\right.$ yield) orange powder ${ }^{1} \mathrm{H}$ NMR $(500 \mathrm{MHz}$, Chloroform- $d$ ) $\delta 8.35(\mathrm{~s}, 1 \mathrm{H})$, $8.10(\mathrm{~d}, J=8.7 \mathrm{~Hz}, 2 \mathrm{H}), 7.87-7.71(\mathrm{~m}, 2 \mathrm{H}), 7.04(\mathrm{~d}, J=8.7 \mathrm{~Hz}, 2 \mathrm{H}), 6.65(\mathrm{~s}, 1 \mathrm{H}), 4.35$ $-4.22(\mathrm{~m}, 2 \mathrm{H}), 3.84-3.73(\mathrm{~m}, 4 \mathrm{H}), 2.91$ (s, 2H), $2.66(\mathrm{~s}, 4 \mathrm{H})$. ESI-MS [M+H]: Calc: 352.39 Found: 353.32

(E)-2-(furan-2-carbonyl)-3-(4-(isopentyloxy)phenyl)acrylonitrile (3217)

$26.1 \mathrm{mg}\left(42.5 \%\right.$ yield) orange powder ${ }^{1} \mathrm{H}$ NMR $(500 \mathrm{MHz}$, Chloroform- $d) \delta 8.35(\mathrm{~s}, 1 \mathrm{H})$, $8.10(\mathrm{~d}, J=8.6 \mathrm{~Hz}, 2 \mathrm{H}), 7.77(\mathrm{~d}, J=14.0 \mathrm{~Hz}, 2 \mathrm{H}), 7.02(\mathrm{~d}, J=8.6 \mathrm{~Hz}, 2 \mathrm{H}), 6.65(\mathrm{~s}, 1 \mathrm{H})$, $4.11(\mathrm{t}, J=6.5 \mathrm{~Hz}, 3 \mathrm{H}), 1.87(\mathrm{dt}, J=13.2,6.6 \mathrm{~Hz}, 2 \mathrm{H}), 1.74(\mathrm{q}, J=6.5 \mathrm{~Hz}, 3 \mathrm{H}), 1.00(\mathrm{~d}$, $J=6.6 \mathrm{~Hz}, 6 \mathrm{H})$. ESI-MS [M+H]: Calc: 309.37 Found: 310.1

\section{MmpL3 Methods}

\section{Protein binding study}

Plasma protein binding was determined by equilibrium dialysis. The red device inserts are supplied ready to use (Thermo Scientific, Rockford, USA) containing plasma and buffer chambers for dialysis. The inserts were placed in base plate. Two different concentrations $(500 \mathrm{ng} / \mathrm{mL}$ and $5 \mu \mathrm{g} / \mathrm{mL}$ ) of Lee 1804 were prepared in rat plasma and an aliquot of $300 \mu \mathrm{L}$ was added in the plasma chamber in duplicate. A $500 \mu \mathrm{L}$ aliquot of PBS was added in the buffer chamber for dialysis. The base plate was covered with sealing tape and incubated at $37^{\circ} \mathrm{C}$ at approximately $100 \mathrm{rpm}$ on an orbital shaker for 4 hours to achieve equilibrium. After incubation $50 \mu \mathrm{L}$ of each post-dialysis samples were pipette out from the plasma and buffer chambers into separate micro-centrifuge tubes. 50 $\mu \mathrm{L}$ of plasma was added to the buffer samples and an equal volume of PBS to the collected plasma samples and vortex. Pipette out $50 \mu \mathrm{L}$ and analyzed for bound and unbound drug concentrations by LC-MS/MS 


\begin{abstract}
Animals
Catheterized male Sprague-Dawley rats (femoral vein for drug administration and jugular vein for blood sample collection) from Harlan Bioscience (Indianapolis, IN), weighing approximately $200 \mathrm{~g}$, were kept on a $12 \mathrm{~h}$ light/ dark cycle with access to food and water ad libitum. The study protocol was approved by the Institutional Animal Care and Use Committee of the University of Tennessee Health Science Center.
\end{abstract}

\title{
Drug formulation and administration
}

Formulation of $1804(10 \mathrm{mg} / \mathrm{kg})$ was prepared by dissolving the drug in $40 \%$ DMSO, $40 \%$ Propylene glycol and 20\% PEG 300. The i.v. doses were administered via femoral vein catheter followed by flushing the catheter with locking solution. A group of 5 rats were dosed for each of the compound.

\section{Sampling procedure}

Blood samples $(250 \mu \mathrm{L})$ were collected at $0.25,0.5,0.75,1.0,1.5,2.0,4.0,6.0$, 8.0, 12.0, 24.0, 36.0 and $48.0 \mathrm{~h}$ after drug administration for all the three compounds. Plasma was separated immediately by centrifugation $\left(10,000 \times \mathrm{g}\right.$ for $10 \mathrm{~min}$ at $\left.4^{\circ} \mathrm{C}\right)$ and stored at $-20^{\circ} \mathrm{C}$ until analysis. Urine samples were collected at an interval of $0-6,6-12$, $12-24,24-36,36-48,48-60$ and $60-72 \mathrm{~h}$ post-dose and stored at $-20^{\circ} \mathrm{C}$ until analysis.

\section{In-vivo pharmacokinetics}

Catheterized male Sprague-Dawley rats (jugular vein for blood sample collection) from Harlan Bioscience (Indianapolis, IN), weighing approximately 190-230 g, were kept on a $12 \mathrm{~h}$ light/ dark cycle with access to food and water ad libitum. Prior to the dosing the animals were fasted overnight and given access to water ad libitum. The study protocol was approved by the Institutional Animal Care and Use Committee of the University of Tennessee Health Science Center.

\section{Drug formulation and administration}

Formulations of Lee $1804(100 \mathrm{mg} / \mathrm{kg}$ ) were prepared in 10\% Vitamin E TPGS (vitamin E d-alpha tocopheryl polyethyleneglycol 1000 succinate). The oral doses were administered using oral gavage needle fitted to $1.0 \mathrm{~mL}$ syringe. A group of 5 rats were dosed for each of the compound.

\section{LC-MS-MS}

Chromatographic separations were carried out using a Shimadzu liquid chromatograph (Shimadzu Corporation, USA) consisting of two pumps, online degasser, system controller and a CTC Leap auto sampler (Leap Technologies, Carrboro, NC). Mobile phase consist of $0.1 \%$ Formic acid spiked acetonitrile (Pump B) and $0.1 \%$ Formic acid spiked HPLC grade water (Pump A) was used at a flow rate of $0.6 \mathrm{~mL} / \mathrm{min}$ in gradient mode. A Phenomenex ${ }^{\circledR} \mathrm{C} 18(2), 3 \mu \mathrm{m}, 50 \times 2.0 \mathrm{~mm}$ column (Phenomenex, Torrance, CA) protected with a guard column was used for the separation. The samples 
$(20 \mu \mathrm{L})$ were injected on column and the eluate was led directly into a mass spectrometer.

\section{UPLC/MS/UV}

LC-MS chromasolv grade acetonitrile (ACN) was purchased from Fisher Scientific (Loughborough, UK). LC-MS chromasolv grade methanol, ammonium bicarbonate and formic acid were obtained from Sigma-Aldrich (St. Louis, MO). Milli-Q water as an ultrapure laboratory grade water was used in aqueous mobile phase. Chromatographic separation was performed on an Acquity UPLC BEH C18 1.7 $\mu \mathrm{m}, 2.1$ x $50 \mathrm{~mm}$ column (Waters Corporation, Milford, MA) using an Acquity ultra performance liquid chromatography system. Data were acquired using Masslynx v. 4.1 and analyzed using the Quanlynx software suite. This was coupled to an SQ mass spectrometer. The total flow rate was $1.0 \mathrm{~mL} / \mathrm{min}$. The sample injection volume was $10 \mu \mathrm{L}$. The UPLC column was maintained at $55^{\circ} \mathrm{C}$. Samples were separated on the column under acidic or basic mobile phase based on their physical-chemical properties. For the acidic mobile phase, solvent A was $0.1 \%$ formic acid in MilliQ $\mathrm{H} 2 \mathrm{O}$ and solvent B was $0.1 \%$ formic acid in ACN. For the basic mobile phase, solvent A was $10 \mathrm{mM}$ ammonium bicarbonate in MilliQ H2O and solvent B was methanol. Samples were eluted from the column under one of the gradients listed in table 1 . The mass spectrometer was operated in positive-ion mode with electrospray ionization. The conditions were as follows: capillary voltage 3.4 $\mathrm{kV}$, cone voltage $30 \mathrm{~V}$, source temperature $130{ }^{\circ} \mathrm{C}$, desolvation temperature $400{ }^{\circ} \mathrm{C}$, desolvation gas $800 \mathrm{~L} / \mathrm{hr}$, cone gas $100 \mathrm{~L} / \mathrm{hr}$. A full scan range from $\mathrm{m} / \mathrm{z}=110-1000$ in $0.2 \mathrm{~s}$ was used to acquire MS data. A single ion recording mass spectrometry for each compound was used to determine the quantification of the samples.

\section{Synthesis of urea compounds: General method}

In a dry round bottom flask equipped with a stir bar, $0.5 \mathrm{mmol}$ of triphosgene was dissolved in three $\mathrm{mL}$ of anhydrous dichloromethane under nitrogen. To this was added dropwise over ten minutes, a solution of $1.350 \mathrm{mmol}$ Isocamphonyl amine, $1.500 \mathrm{mmol}$ of diisopropylethylamine, and three $\mathrm{mL}$ of anhydrous dichloromethane. This reaction was allowed to stir at room temperature for fifteen minutes while the next reaction was run. In a separate dry round bottom flask, the appropriate amine was dissolved in $15 \mathrm{~mL}$ of THF. Flask was cooled to $-78^{\circ} \mathrm{C}$ and $1 \mathrm{mmol}$ of $2.5 \mathrm{M}$ Butyl Lithium in hexane was added slowly. Reaction was allowed to stir while coming to room temperature for 20 minutes. After 20 minutes the first reaction was added carefully to the second and combined reaction was allowed to react for one hour before remaining Butyl Lithium was quenched with methanol. Solvent removed under vacuum and product was purified via flash chromatography (Hexane to Ethyl Acetate).

Below is a listing of analytical purity of compounds discussed in this section. Experimental details for determination are described in the methods above. 
1-(5-(tert-butyl)isoxazol-3-yl)-3-((1R,2R,3R,5S)-2,6,6-trimethylbicyclo[3.1.1]heptan-3yl)urea: (1)

Yield: $22.7 \mathrm{mg}$ (14.07\%) of white powder M.P. $145-147{ }^{\circ} \mathrm{C} ;{ }^{1} \mathrm{H}$ NMR $(400 \mathrm{MHz}$, Chloroform-d) $\delta 9.12(\mathrm{~s}, 1 \mathrm{H}), 7.14(\mathrm{~s}, 1 \mathrm{H}), 5.93(\mathrm{~s}, 1 \mathrm{H}), 4.22(\mathrm{~h}, J=7.1,6.5 \mathrm{~Hz}, 1 \mathrm{H})$, 2.65 (ddt, $J=13.3,10.2,2.6 \mathrm{~Hz}, 1 \mathrm{H}), 2.70-2.59(\mathrm{~m}, 0 \mathrm{H}), 2.43$ (ddt, $J=8.5,6.3,3.9 \mathrm{~Hz}$, $1 \mathrm{H}), 2.03-1.91(\mathrm{~m}, 2 \mathrm{H}), 2.02-1.92(\mathrm{~m}, 2 \mathrm{H}), 1.86(\mathrm{dt}, J=5.9,3.0 \mathrm{~Hz}, 1 \mathrm{H}), 1.86(\mathrm{t}, J=$ $5.9 \mathrm{~Hz}, 1 \mathrm{H}), 1.71$ (ddd, $J=14.0,6.1,2.4 \mathrm{~Hz}, 1 \mathrm{H}), 1.34(\mathrm{~s}, 9 \mathrm{H}), 1.25$ (s, 3H), 1.17 (d, $J=$ $7.1 \mathrm{~Hz}, 3 \mathrm{H}), 1.07$ (s, 3H), 1.00 (d, $J=9.8 \mathrm{~Hz}, 1 \mathrm{H})$; ESI-HRMS m/z: [M+H]+ calcd: 320.2293, found: 320.2327

1-(5-methylisoxazol-3-yl)-3-((1R,2R,3R,5S)-2,6,6-trimethylbicyclo[3.1.1]heptan-3yl) urea (2)

Yield: $71.3 \mathrm{mg}$ (51.4\%) of an off-white powder; M.P: $133-138^{\circ} \mathrm{C} ;{ }^{1} \mathrm{H}$ NMR $(400 \mathrm{MHz}$, Chloroform-d) $\delta 9.50(\mathrm{~s}, 1 \mathrm{H}), 7.07(\mathrm{~s}, 1 \mathrm{H}), 6.01(\mathrm{~s}, 1 \mathrm{H}), 4.27-4.13(\mathrm{~m}, 1 \mathrm{H}), 2.63$ (dddd, $J=13.8,9.9,3.6,2.1 \mathrm{~Hz}, 1 \mathrm{H}), 2.43$ (dtd, $J=9.8,6.0,2.2 \mathrm{~Hz}, 1 \mathrm{H}), 2.37$ (s, 3H), $2.03-$

$1.90(\mathrm{~m}, 2 \mathrm{H}), 1.85(\mathrm{td}, J=5.8,1.9 \mathrm{~Hz}, 1 \mathrm{H}), 1.69(\mathrm{ddd}, J=14.0,6.1,2.4 \mathrm{~Hz}, 1 \mathrm{H}), 1.25$ (s, $3 \mathrm{H}), 1.16(\mathrm{~d}, J=7.2 \mathrm{~Hz}, 3 \mathrm{H}), 1.06$ (s, 3H), 0.98 (d, $J=9.8 \mathrm{~Hz}, 1 \mathrm{H})$; ESI-HRMS m/z: $[\mathrm{M}+\mathrm{H}]+$ Calcd: 278.1824 , found: 278.1879

1-(4-methylthiazol-2-yl)-3-((1R,2R,3R,5S)-2,6,6-trimethylbicyclo[3.1.1]heptan-3yl)urea: (3)

Yield: $76.7 \mathrm{mg}$ (51.8\%) of colorless oil; M.P. N/A; ${ }^{1} \mathrm{H}$ NMR (400 MHz, Chloroform- $d$ ) $\delta$ $6.76(\mathrm{~s}, 1 \mathrm{H}), 4.02(\mathrm{q}, J=7.5,7.0 \mathrm{~Hz}, 1 \mathrm{H}), 3.32(\mathrm{~s}, 2 \mathrm{H}), 2.46(\mathrm{t}, J=11.9 \mathrm{~Hz}, 1 \mathrm{H}), 2.18(\mathrm{~s}$, $3 \mathrm{H}), 1.76-1.64(\mathrm{~m}, 2 \mathrm{H}), 1.50(\mathrm{dd}, J=13.9,6.1 \mathrm{~Hz}, 1 \mathrm{H}), 1.06(\mathrm{~s}, 3 \mathrm{H}), 0.98(\mathrm{~d}, J=7.2$ $\mathrm{Hz}, 3 \mathrm{H}), 0.87$ (s, 3H), $0.76(\mathrm{~d}, J=9.8 \mathrm{~Hz}, 1 \mathrm{H})$; ESI-HRMS m/z: [M+H]+ Calcd: 294.1595, found: 294.1626

1-(5-methylthiazol-2-yl)-3-((1R,2R,3R,5S)-2,6,6-trimethylbicyclo[3.1.1]heptan-3yl)urea: (4)

Yield: 91.6mg (62.4\%) of colorless oil; M.P. N/A; ${ }^{1} \mathrm{H}$ NMR (400 MHz, Chloroform- $d$ ) $\delta$ $10.31(\mathrm{~s}, 1 \mathrm{H}), 6.35(\mathrm{~s}, 1 \mathrm{H}), 4.35-4.11(\mathrm{~m}, 1 \mathrm{H}), 3.51(\mathrm{~s}, 1 \mathrm{H}), 2.67$ (dddd, $J=13.7,10.0$, 3.7, 2.2 Hz, 1H), $2.51-2.39(\mathrm{~m}, 1 \mathrm{H}), 2.35(\mathrm{~d}, J=1.1 \mathrm{~Hz}, 3 \mathrm{H}), 2.05-1.95(\mathrm{~m}, 1 \mathrm{H}), 2.00$ $-1.82(\mathrm{~m}, 2 \mathrm{H}), 1.70(\mathrm{ddd}, J=13.9,6.1,2.4 \mathrm{~Hz}, 1 \mathrm{H}), 1.26(\mathrm{~s}, 3 \mathrm{H}), 1.18(\mathrm{~d}, J=7.2 \mathrm{~Hz}$, 3H), 1.07 (s, 3H), 0.94 (d, $J=9.8$ Hz, 1H). ESI-HRMS m/z: [M+H]+ Calcd: 294.1595, found: 294.1623

1-(thiazol-2-yl)-3-((1R,2R,3R,5S)-2,6,6-trimethylbicyclo[3.1.1]heptan-3-yl)urea (5) Yield: N/A; M.P. N/A; ${ }^{1} \mathrm{H}$ NMR (400 MHz, Chloroform- $d$ ) $\delta 11.24$ (s, 1H), 7.35 (dd, $J$ $=3.7,1.4 \mathrm{~Hz}, 1 \mathrm{H}), 6.82(\mathrm{~d}, J=3.7 \mathrm{~Hz}, 0 \mathrm{H}), 4.25(\mathrm{p}, J=7.5,7.0 \mathrm{~Hz}, 1 \mathrm{H}), 2.73-2.58(\mathrm{~m}$, $1 \mathrm{H}), 2.51-2.40(\mathrm{~m}, 1 \mathrm{H}), 2.05-1.82(\mathrm{~m}, 3 \mathrm{H}), 1.71(\mathrm{dd}, J=14.0,6.3,2.3 \mathrm{~Hz}, 1 \mathrm{H}), 1.26$ $(\mathrm{d}, J=1.5 \mathrm{~Hz}, 4 \mathrm{H}), 1.19(\mathrm{dd}, J=7.3,1.6 \mathrm{~Hz}, 3 \mathrm{H}), 1.18-1.02(\mathrm{~m}, 4 \mathrm{H}), 0.96(\mathrm{~d}, J=9.9$ $\mathrm{Hz}, 1 \mathrm{H})$; ESI-HRMS m/z: N/A

1-(benzo[d]thiazol-2-yl)-3-((1R,2R,3R,5S)-2,6,6-trimethylbicyclo[3.1.1]heptan-3yl)urea (7)

Yield: 117.2 (71.1\%) of a white powder; M.P. $185-187^{\circ} \mathrm{C} ;{ }^{1} \mathrm{H}$ NMR $(400 \mathrm{MHz}$, Chloroform- $d$ ) $\delta 11.32(\mathrm{~s}, 1 \mathrm{H}), 7.78(\mathrm{dd}, J=12.2,8.0 \mathrm{~Hz}, 2 \mathrm{H}), 7.42(\mathrm{ddd}, J=8.3,7.3$, $1.3 \mathrm{~Hz}, 1 \mathrm{H}), 7.27$ (ddd, $J=8.3,7.3,1.1 \mathrm{~Hz}, 1 \mathrm{H}), 4.37-4.24(\mathrm{~m}, 1 \mathrm{H}), 2.77-2.65$ (m, 
$1 \mathrm{H}), 2.51-2.40(\mathrm{~m}, 1 \mathrm{H}), 2.07(\mathrm{~s}, 1 \mathrm{H}), 2.11-1.96(\mathrm{~m}, 1 \mathrm{H}), 1.96-1.84(\mathrm{~m}, 1 \mathrm{H}), 1.74$ (ddd, $J=14.1,6.2,2.4 \mathrm{~Hz}, 1 \mathrm{H}), 1.29(\mathrm{~d}, J=7.2 \mathrm{~Hz}, 1 \mathrm{H}), 1.26(\mathrm{~d}, 3 \mathrm{H}), 1.22(\mathrm{~d}, J=7.2$ $\mathrm{Hz}, 3 \mathrm{H}), 1.08$ (s, 3H), $1.02-0.83$ (m, 2H). ESI-HRMS m/z: [M+H]+ Calcd: 330.1595, found: 330.1654

1-(5-cyclobutyl-1,3,4-oxadiazol-2-yl)-3-((1R,2R,3R,5S)-2,6,6trimethylbicyclo[3.1.1]heptan-3-yl)urea (8)

Yield: 78.0mg (49\%) of brown oil; M.P. N/A; ${ }^{1} \mathrm{H}$ NMR (400 MHz, Chloroform- $d$ ) $\delta$ $10.34(\mathrm{~s}, 1 \mathrm{H}), 8.16(\mathrm{~s}, 1 \mathrm{H}), 4.31-4.18(\mathrm{~m}, 1 \mathrm{H}), 3.65(\mathrm{p}, J=8.3 \mathrm{~Hz}, 1 \mathrm{H}), 2.70-2.57(\mathrm{~m}$, $1 \mathrm{H}), 2.50-2.34(\mathrm{~m}, 5 \mathrm{H}), 2.18-1.93(\mathrm{~m}, 4 \mathrm{H}), 1.90-1.80(\mathrm{~m}, 1 \mathrm{H}), 1.73(\mathrm{ddd}, J=14.0$, 6.0, 2.4 Hz, 1H), 1.25 (s, 3H), 1.17 (d, J=7.2 Hz, 3H), 1.07 (s, 3H), $1.06-1.00(\mathrm{~m}, 1 \mathrm{H})$. ESI-HRMS m/z: [M+H]+ Calcd: 319.2089, found 319.2154

\section{1-(5-isopropyl-1,3,4-oxadiazol-2-yl)-3-((1R,2R,3R,5S)-2,6,6-}

trimethylbicyclo[3.1.1]heptan-3-yl)urea (9)

Yield: $9.0 \mathrm{mg}$ (5.87\%) of Yellow oil; M.P. N/A; ${ }^{1} \mathrm{H}$ NMR $(400 \mathrm{MHz}$, Chloroform- $d) \delta$ $8.01(\mathrm{~s}, 1 \mathrm{H}), 7.28$ (s, 3H), 4.23 (p, $J=7.7,7.3 \mathrm{~Hz}, 1 \mathrm{H}), 3.12$ (p, $J=6.8 \mathrm{~Hz}, 1 \mathrm{H}), 2.65$ (t, $J$ $=12.1 \mathrm{~Hz}, 1 \mathrm{H}), 2.45(\mathrm{~s}, 1 \mathrm{H}), 1.99(\mathrm{~d}, J=6.8 \mathrm{~Hz}, 2 \mathrm{H}), 1.87(\mathrm{~s}, 1 \mathrm{H}), 1.72(\mathrm{dd}, J=14.0,5.9$ $\mathrm{Hz}, 1 \mathrm{H}), 1.39$ (dd, $J=7.1,1.9 \mathrm{~Hz}, 5 \mathrm{H}), 1.26(\mathrm{~d}, J=2.0 \mathrm{~Hz}, 3 \mathrm{H}), 1.17$ (dd, $J=7.2,1.9$ $\mathrm{Hz}, 3 \mathrm{H}), 1.08(\mathrm{~d}, J=1.8 \mathrm{~Hz}, 3 \mathrm{H}), 1.01(\mathrm{~d}, J=9.9 \mathrm{~Hz}, 1 \mathrm{H})$. ESI-HRMS m/z: [M+H]+ Calcd: 307.2089 , found: 307.2140

\section{Methyl 2-hydroxy-4-(3-((1R,2R,3R,5S)-2,6,6-trimethylbicyclo[3.1.1]heptan-3-} yl)ureido)benzoate (10)

Yield: $86.7 \mathrm{mg}(50.1 \%)$ of clear film on glass; M.P. N/A; ${ }^{1} \mathrm{H}$ NMR $(400 \mathrm{MHz}$, Chloroform- $d) \delta 10.60(\mathrm{~s}, 1 \mathrm{H}), 7.48(\mathrm{~d}, J=8.8 \mathrm{~Hz}, 1 \mathrm{H}), 6.87-6.75(\mathrm{~m}, 2 \mathrm{H}), 6.67(\mathrm{~d}, J=$ $2.1 \mathrm{~Hz}, 1 \mathrm{H}), 4.91(\mathrm{~s}, 1 \mathrm{H}), 3.69(\mathrm{~s}, 3 \mathrm{H}), 3.67(\mathrm{~s}, 1 \mathrm{H}), 3.29(\mathrm{~s}, 1 \mathrm{H}), 2.47-2.34(\mathrm{~m}, 1 \mathrm{H})$, $2.21-2.10(\mathrm{~m}, 1 \mathrm{H}), 1.74-1.67(\mathrm{~m}, 1 \mathrm{H}), 1.62-1.55(\mathrm{~m}, 1 \mathrm{H}), 1.56-1.47(\mathrm{~m}, 1 \mathrm{H}), 1.41$ $-1.30(\mathrm{~m}, 1 \mathrm{H}), 0.99(\mathrm{~s}, 2 \mathrm{H}), 0.91(\mathrm{~d}, J=7.2 \mathrm{~Hz}, 3 \mathrm{H}), 0.78(\mathrm{~s}, 3 \mathrm{H}), 0.56(\mathrm{~d}, J=9.9 \mathrm{~Hz}$, 1H). ESI-HRMS m/z: [M+H]+ Calcd: 347.1926, found: 347.1956

\section{1-(5-methyl-1-propyl-1H-pyrazol-3-yl)-3-((1R,2R,3R,5S)-2,6,6-}

trimethylbicyclo[3.1.1]heptan-3-yl)urea (11)

Yield: 46.4mg (29.1\%) of off-white powder; M.P. $139-141{ }^{\circ} \mathrm{C} ;{ }^{1} \mathrm{H}$ NMR (400 MHz, Chloroform-d) $\delta 7.86(\mathrm{~s}, 1 \mathrm{H}), 6.99(\mathrm{~s}, 1 \mathrm{H}), 5.55(\mathrm{~s}, 1 \mathrm{H}), 4.20$ (dddd, $J=9.9,8.3,6.8,5.8$ $\mathrm{Hz}, 1 \mathrm{H}), 3.87$ (t, $J=6.9 \mathrm{~Hz}, 2 \mathrm{H}), 2.67$ (dddd, $J=13.7,9.9,3.6,2.2 \mathrm{~Hz}, 1 \mathrm{H}), 2.43$ (dtd, $J$ $=9.7,6.1,2.2 \mathrm{~Hz}, 1 \mathrm{H}), 2.22(\mathrm{~d}, J=0.7 \mathrm{~Hz}, 3 \mathrm{H}), 2.03-1.94(\mathrm{~m}, 1 \mathrm{H}), 1.91-1.76(\mathrm{~m}$, 5H), 1.68 (ddd, $J=14.0,5.9,2.5 \mathrm{~Hz}, 1 \mathrm{H}), 1.25$ (s, 3H), 1.18 (d, $J=7.2 \mathrm{~Hz}, 3 \mathrm{H}), 1.07$ (s, 3H), 0.94 (t, $J=7.4 \mathrm{~Hz}, 4 \mathrm{H})$. ESI-HRMS m/z: [M+H]+ Calcd:319.2453, found: 319.2536

\section{1-(5-chloropyrimidin-2-yl)-3-((1R,2R,3R,5S)-2,6,6-trimethylbicyclo[3.1.1]heptan-3-} yl) urea (12)

Yield: 33.3mg (21.6\%) of white powder; M.P. $184-185^{\circ} \mathrm{C} ;{ }^{1} \mathrm{H}$ NMR $(400 \mathrm{MHz}$, Chloroform- $d$ ) $\delta 8.57(\mathrm{~d}, J=8.3 \mathrm{~Hz}, 1 \mathrm{H}), 8.41(\mathrm{~s}, 2 \mathrm{H}), 8.02(\mathrm{~s}, 1 \mathrm{H}), 4.25-4.10(\mathrm{~m}, 1 \mathrm{H})$, $2.67-2.55(\mathrm{~m}, 1 \mathrm{H}), 2.42-2.33(\mathrm{~m}, 1 \mathrm{H}), 1.95-1.90(\mathrm{~m}, 1 \mathrm{H}), 1.89-1.83(\mathrm{~m}, 1 \mathrm{H}), 1.83$ -1.77 (m, $1 \mathrm{H}), 1.61$ (ddd, $J=14.0,6.0,2.5 \mathrm{~Hz}, 1 \mathrm{H}), 1.17$ (s, 3H), 1.10 (d, J=7.2 Hz, 
3H), 1.00 (s, 3H), 0.89 (d, 1H). ESI-HRMS m/z: [M+H]+ Calcd: 310.1374, found: 309.1478

1-(5-(tert-butyl)isoxazol-3-yl)-3-((1S,2S,3S,5R)-2,6,6-trimethylbicyclo[3.1.1]heptan-3yl) urea (13)

Yield: 9.2mg (5.76\%) of clear film on glass; M.P. N/A; ${ }^{1} \mathrm{H}$ NMR $(400 \mathrm{MHz}$, Chloroformd) $\delta 8.60(\mathrm{~s}, 1 \mathrm{H}), 7.16(\mathrm{~s}, 1 \mathrm{H}), 5.87(\mathrm{~s}, 1 \mathrm{H}), 4.22(\mathrm{ddt}, J=10.1,8.4,6.6 \mathrm{~Hz}, 1 \mathrm{H}), 2.72-$ $2.59(\mathrm{~m}, 1 \mathrm{H}), 2.49-2.38(\mathrm{~m}, 1 \mathrm{H}), 2.04-1.90(\mathrm{~m}, 2 \mathrm{H}), 1.86(\mathrm{td}, J=5.9,1.8 \mathrm{~Hz}, 1 \mathrm{H})$, 1.71 (ddd, $J=14.0,6.1,2.4 \mathrm{~Hz}, 1 \mathrm{H}), 1.35(\mathrm{~s}, 8 \mathrm{H}), 1.26(\mathrm{~s}, 3 \mathrm{H}), 1.18(\mathrm{~d}, J=7.2 \mathrm{~Hz}, 3 \mathrm{H})$, 1.08 (s, 2H), 1.00 (d, $J=9.8$ Hz, 1H). ESI-HRMS m/z: [M+H]+ Calcd: 320.2293, found: 320.2354

\section{1-(5-methylisoxazol-3-yl)-3-((1S,2S,3S,5R)-2,6,6-trimethylbicyclo[3.1.1]heptan-3- yl) urea (14)}

Yield: 21.9mg (15.8\%) of off white powder; M.P. N/A; ${ }^{1} \mathrm{H}$ NMR (400 MHz, Chloroformd) $\delta 8.52(\mathrm{~s}, 1 \mathrm{H}), 7.12(\mathrm{~s}, 1 \mathrm{H}), 5.92(\mathrm{~s}, 1 \mathrm{H}), 4.26-4.15(\mathrm{~m}, 1 \mathrm{H}), 2.70-2.59(\mathrm{~m}, 1 \mathrm{H})$, $2.47-2.40(\mathrm{~m}, 1 \mathrm{H}), 2.39(\mathrm{~s}, 3 \mathrm{H}), 2.04-1.90(\mathrm{~m}, 2 \mathrm{H}), 1.86(\mathrm{td}, J=5.9,1.9 \mathrm{~Hz}, 1 \mathrm{H}), 1.70$ (ddd, $J=14.0,6.0,2.4 \mathrm{~Hz}, 1 \mathrm{H}), 1.26$ (s, 3H), 1.17 (d, $J=7.2 \mathrm{~Hz}, 3 \mathrm{H}), 1.08$ (s, 3H), 1.00 $(\mathrm{d}, J=9.9 \mathrm{~Hz}, 1 \mathrm{H})$. Calcd: 278.1824 , found: 278.1881

\section{1-(4-methylthiazol-2-yl)-3-((1S,2S,3S,5R)-2,6,6-trimethylbicyclo[3.1.1]heptan-3-yl)urea (15)}

Yield: $26.3 \mathrm{mg}(17.93 \%)$ of yellow oil; M.P. N/A; ${ }^{1} \mathrm{H}$ NMR (400 MHz, Chloroform- $d$ ) $\delta$ $6.15(\mathrm{~s}, 1 \mathrm{H}), 4.16-3.89(\mathrm{~m}, 1 \mathrm{H}), 2.47$ (dddd, $J=14.0,10.1,3.8,2.3 \mathrm{~Hz}, 1 \mathrm{H}), 2.30-$ $2.18(\mathrm{~m}, 1 \mathrm{H}), 2.14(\mathrm{~s}, 3 \mathrm{H}), 1.83-1.62(\mathrm{~m}, 3 \mathrm{H}), 1.49(\mathrm{ddd}, J=14.1,6.1,2.4 \mathrm{~Hz}, 1 \mathrm{H})$, 1.06 (s, 2H), 0.98 (d, $J=7.1,1.2 \mathrm{~Hz}, 3 \mathrm{H}), 0.86(\mathrm{~s}, 2 \mathrm{H}), 0.74$ (d, $J=9.7 \mathrm{~Hz}, 1 \mathrm{H})$. ESIHRMS m/z: [M+H]+ Calcd: 294.1595, found: 294.1659

1-(5-cyclobutyl-1,3,4-oxadiazol-2-yl)-3-((1S,2S,3S,5R)-2,6,6trimethylbicyclo[3.1.1]heptan-3-yl)urea (17)

Yield: $54.8 \mathrm{mg}$ (34.4\%) of Pink oil; M.P. N/A; ${ }^{1} \mathrm{H}$ NMR (400 MHz, Chloroform- $d$ ) $\delta$ $9.71(\mathrm{~s}, 1 \mathrm{H}), 8.13(\mathrm{~d}, J=8.0 \mathrm{~Hz}, 1 \mathrm{H}), 4.28-4.18(\mathrm{~m}, 1 \mathrm{H}), 3.65(\mathrm{p}, J=8.4 \mathrm{~Hz}, 1 \mathrm{H}), 2.65$ (dddd, $J=13.8,10.0,3.7,2.2 \mathrm{~Hz}, 1 \mathrm{H}), 2.51-2.36(\mathrm{~m}, 5 \mathrm{H}), 2.22-1.97(\mathrm{~m}, 3 \mathrm{H}), 1.87$ $(\mathrm{td}, J=5.9,1.9 \mathrm{~Hz}, 1 \mathrm{H}), 1.74(\mathrm{ddd}, J=14.0,6.1,2.4 \mathrm{~Hz}, 1 \mathrm{H}), 1.26(\mathrm{~s}, 3 \mathrm{H}), 1.18(\mathrm{~d}, J=$ $7.2 \mathrm{~Hz}, 3 \mathrm{H}), 1.08(\mathrm{~s}, 3 \mathrm{H}), 1.03(\mathrm{~d}, J=9.8 \mathrm{~Hz}, 1 \mathrm{H})$. ESI-HRMS m/z: $[\mathrm{M}+\mathrm{H}]+$ Calcd: 319.2089, found: 319.2153

\section{1-(5-isopropyl-1,3,4-oxadiazol-2-yl)-3-((1S,2S,3S,5R)-2,6,6-}

trimethylbicyclo[3.1.1]heptan-3-yl)urea (18)

Yield: $55.7 \mathrm{mg}(36.4 \%)$ of tan powder; M.P. $148-156{ }^{\circ} \mathrm{C}$; ${ }^{1} \mathrm{H}$ NMR $(400 \mathrm{MHz}$, Chloroform-d) $\delta 6.82(\mathrm{~s}, 1 \mathrm{H}), 4.96(\mathrm{~s}, 1 \mathrm{H}), 4.15(\mathrm{~d}, J=8.3 \mathrm{~Hz}, 1 \mathrm{H}), 2.66-2.57(\mathrm{~m}, 1 \mathrm{H})$, $2.44-2.34(\mathrm{~m}, 1 \mathrm{H}), 1.99-1.91(\mathrm{~m}, 1 \mathrm{H}), 1.86-1.78(\mathrm{~m}, 1 \mathrm{H}), 1.77-1.66(\mathrm{~m}, 1 \mathrm{H}), 1.57$ (ddd, $J=13.9,6.1,2.4 \mathrm{~Hz}, 1 \mathrm{H}), 1.23$ (s, 4H), 1.14 (d, $J=7.1 \mathrm{~Hz}, 4 \mathrm{H}), 1.01$ (s, 3H), 0.78 $(\mathrm{d}, J=9.7 \mathrm{~Hz}, 1 \mathrm{H})$. ESI-HRMS m/z: [M+H]+ Calcd: 307.2089, found: 307.2138 


\section{Isocyanate preparation of ureas}

In a round bottom flask equipped with a stir bar, Isocamphonyl amine (1 mmol) was added to DCM $(10 \mathrm{ml})$. To this solution, appropriate isocyanate $(1.2 \mathrm{mmol}$ was added followed by triethylamine $(3.60 \mathrm{mmol})$ was added. The reaction was stirred at room temperature overnight. Final product separated by a Hexane to Ethyl Acetate gradient flash column.

Below is a listing of analytical purity of compounds discussed in this section. Experimental details for determination are described in the methods above.

1-(4-chlorophenyl)-3-((1R,2R,3R,5S)-2,6,6-trimethylbicyclo[3.1.1]heptan-3-yl)urea (6) Yield: $304.3 \mathrm{mg}$ (98.7\%) of yellow oil; M.P: N/A; ${ }^{1} \mathrm{H}$ NMR (400 MHz, Chloroform- $d$ ) $\delta$ $7.68(\mathrm{~s}, 1 \mathrm{H}), 7.28-7.24(\mathrm{~m}, 2 \mathrm{H}), 7.22-7.13(\mathrm{~m}, 2 \mathrm{H}), 4.16-4.03(\mathrm{~m}, 1 \mathrm{H}), 3.51(\mathrm{~d}, J=$ $5.3 \mathrm{~Hz}, 2 \mathrm{H}), 2.62-2.50(\mathrm{~m}, 1 \mathrm{H}), 2.40-2.29(\mathrm{~m}, 1 \mathrm{H}), 1.93-1.86(\mathrm{~m}, 1 \mathrm{H}), 1.77(\mathrm{td}, J=$ $5.8,1.8 \mathrm{~Hz}, 1 \mathrm{H}), 1.67$ (pd, $J=7.2,1.8 \mathrm{~Hz}, 1 \mathrm{H}), 1.60-1.48(\mathrm{~m}, 2 \mathrm{H}), 1.20(\mathrm{~s}, 3 \mathrm{H}), 1.09$ $(\mathrm{d}, J=7.2 \mathrm{~Hz}, 3 \mathrm{H}), 0.93(\mathrm{~s}, 3 \mathrm{H}), 0.72(\mathrm{~d}, J=9.8 \mathrm{~Hz}, 1 \mathrm{H})$. ESI-HRMS m/z: $[\mathrm{M}+\mathrm{H}]+$ Calcd: 308.1469 , found: 307.1559

1-(4-chlorophenyl)-3-((1S,2S,3S,5R)-2,6,6-trimethylbicyclo[3.1.1]heptan-3-yl)urea (16) Yield: $264.7 \mathrm{mg}(86 \%)$ of yellow oil; M.P: N/A; ${ }^{1} \mathrm{H}$ NMR (400 MHz, Chloroform- $\left.d\right) \delta$ $7.33-7.20(\mathrm{~m}, 4 \mathrm{H}), 6.82(\mathrm{~s}, 1 \mathrm{H}), 4.96(\mathrm{~s}, 1 \mathrm{H}), 4.15(\mathrm{~d}, J=8.3 \mathrm{~Hz}, 1 \mathrm{H}), 2.66-2.57(\mathrm{~m}$, $1 \mathrm{H}), 2.45-2.34(\mathrm{~m}, 1 \mathrm{H}), 1.95(\mathrm{dt}, J=6.3,3.0 \mathrm{~Hz}, 1 \mathrm{H}), 1.82(\mathrm{td}, J=5.9,1.8 \mathrm{~Hz}, 1 \mathrm{H})$, 1.71 (q, $J=7.0 \mathrm{~Hz}, 1 \mathrm{H}), 1.57$ (ddd, $J=13.9,6.1,2.4 \mathrm{~Hz}, 1 \mathrm{H}), 1.23$ (s, 3H), 1.14 (d, $J=$ $7.1 \mathrm{~Hz}, 3 \mathrm{H}), 1.01(\mathrm{~s}, 2 \mathrm{H}), 0.78(\mathrm{~d}, J=9.7 \mathrm{~Hz}, 1 \mathrm{H})$. ESI-HRMS m/z: [M+H]+ Calcd: 308.1469, found: 307.2136

\section{Preparation of methylene-spaced ureas}

5-(tert-butyl)isoxazol-3-amine (1 mmol) was in solution with $3 \mathrm{~mL}$ of DCM and 2-chloroacetyl chloride $(1.1 \mathrm{mmol})$ and stired for $30 \mathrm{~min}$. Solid was filtered off and purified with flash chromatography (Hexane to ethyl acetate). In a separate reaction sodium hydride (1.24 mmol) was added to 2-Adamantyl Amine Hydrogen Chloride (0.79 $\mathrm{mmol}$ ) in $3 \mathrm{~mL}$ of DMF and was also stirred for $30 \mathrm{~min}$. After $30 \mathrm{~min}$ the Isoxazol reaction was added and the combined reactions were allowed to stir for 30 min at $35^{\circ} \mathrm{C}$. Water $(10 \mathrm{~mL})$ was added to the crude mixture and product was extracted with Ethyl acetate $(2 \times 15 \mathrm{~mL})$, the organic layer washed with water $(2 \times 20 \mathrm{~mL})$ and dried over $\mathrm{Na} 2 \mathrm{SO} 4$. Solvent removed under vacuum and flash chromatography (Hexane to Ethyl acetate) was used to obtain pure product.

Below is a listing of analytical purity of compounds discussed in this section. Experimental details for determination are described in the methods above.

2-((1r,3r,5r,7r)-adamantan-2-ylamino)-N-(5-(tert-butyl)isoxazol-3-yl)acetamide (19) Yield: 57.4mg (17.32\%) of white powder; M.P. $114-117{ }^{\circ} \mathrm{C} ;{ }^{1} \mathrm{H}$ NMR $(400 \mathrm{MHz}$, Chloroform-d) $\delta 10.00(\mathrm{~s}, 1 \mathrm{H}), 6.72(\mathrm{~s}, 1 \mathrm{H}), 3.44(\mathrm{~s}, 2 \mathrm{H}), 2.71(\mathrm{~d}, J=3.3 \mathrm{~Hz}, 1 \mathrm{H}), 1.96-$ 
$1.77(\mathrm{~m}, 10 \mathrm{H}), 1.75-1.66(\mathrm{~m}, 2 \mathrm{H}), 1.60(\mathrm{dd}, J=12.9,3.4 \mathrm{~Hz}, 2 \mathrm{H}), 1.36(\mathrm{~d}, J=0.5 \mathrm{~Hz}$, 9H). ESI-HRMS m/z: [M+H]+ Calcd: 332.2293, found: 332.2378

2-((5-(tert-butyl)isoxazol-3-yl)amino)-N-((1S,2S,3S,5R)-2,6,6trimethylbicyclo[3.1.1]heptan-3-yl)acetamide (20)

Yield: 46.7mg (14\%) of clear oil; M.P: N/A; ${ }^{1}$ H NMR (400 MHz, Chloroform- $d$ ) $\delta 10.01$ $(\mathrm{s}, 1 \mathrm{H}), 6.71(\mathrm{~s}, 1 \mathrm{H}), 3.45(\mathrm{~d}, J=9.1 \mathrm{~Hz}, 2 \mathrm{H}), 2.96-2.85(\mathrm{~m}, 1 \mathrm{H}), 2.51-2.32(\mathrm{~m}, 2 \mathrm{H})$, $1.97(\mathrm{tt}, J=5.9,3.4 \mathrm{~Hz}, 1 \mathrm{H}), 1.87-1.74(\mathrm{~m}, 2 \mathrm{H}), 1.58(\mathrm{ddd}, J=13.6,5.4,2.6 \mathrm{~Hz}, 1 \mathrm{H})$, $1.35(\mathrm{~s}, 7 \mathrm{H}), 1.38-1.27(\mathrm{~m}, 1 \mathrm{H}), 1.22(\mathrm{~s}, 3 \mathrm{H}), 1.16(\mathrm{~d}, J=7.1 \mathrm{~Hz}, 3 \mathrm{H}), 0.94(\mathrm{~s}, 3 \mathrm{H})$, 0.89 (d, $J=9.8 \mathrm{~Hz}, 1 \mathrm{H})$. ESI-HRMS m/z: N/A 


\section{CHAPTER 3. DESIGN AND REPURPOSING OF ANTI-FOLATE ANTIBIOTICS AND BIOACTIVES}

\section{Anti-Folate Antibiotics}

The easiest way to selectively target bacteria over human cells is to target metabolic pathways required for bacterial growth or survival that either don't exist in humans or are not essential through metabolic redundancies or circumvention. Prontosil is an example of a prodrug that has an active metabolite that inhibits the folate biosynthesis pathway. Folate is critical for the survivability of all living organisms as a co-factor in DNA synthesis via single carbon transfers in purine nucleotide biosynthesis [5]. Humans obtain folates through diet as vitamin B9, whereas bacteria must synthesize folate utilizing the folate biosynthesis pathway (Figure 3-1). This discrepancy in metabolic requirements is where the anti-folate antibiotics become selective and effective in treating a wide variety of pathogens [225]. This transfer of a single carbon atom is often a critical step in the biosynthesis of required nucleobases such as thymine and in the generation of other metabolites including the amino acid methionine.

The primary approach to target folate biosynthesis is the drug combination of TMP and SMX (Bactrim), which also an excellent illustration of antibiotic synergy. By targeting two enzymes in the same biosynthetic cascade this combination slows the emergence of resistance and is able to be more efficacious than the sum of their two activities [3]. SMX is a sulfonamide antibiotic, and as discussed before, is a PABA mimic designed to inhibit DHPS. Inhibition of the folate biosynthesis by sulfonamides has recently been postulated to be via inhibition of downstream enzymes by the incorrectly conjugated product of the sulfonamides to DHPP as well as a depletion of DHPP required for proper DHPS function (Figure 3-2) [226]. This conjecture originated when it was shown that overexpression of DHPS has little effect on SMX MIC [226]. This has led to the synergy theory that SMX not only forms an inactive conjugate with DHPP that alleviates competitive binding for TMP at the DHFR, but that this conjugate could in fact be inhibiting later enzymes in this biosynthetic cascade. Bactrim (SMX:TMP) is one of the only generic orally bioavailable treatments for MRSA and is useful for a number of infections in a plethora of tissue sites. TMP is often considered the more potent of the two in combination, and in most bacterial species it does in fact have the highest antibacterial potency. However in the yeast-like fungus Pnuemocystis jirovecii, the causative agent of Pneumocystis pneumonia (PCP), that trend is reversed. Bactrim is the common front line prophylaxis therapy used to prevent PCP [227], an opportunistic lung infection that takes hold of immune suppressed patients, and it has been shown that SMX provides most of the antimicrobial activity [228].

\section{Chapter Outline}

In this chapter my efforts are outlined towards the discovery of new anti-folate agents in two mini studies: (i) A structure guided synthesis project for novel HPPK 


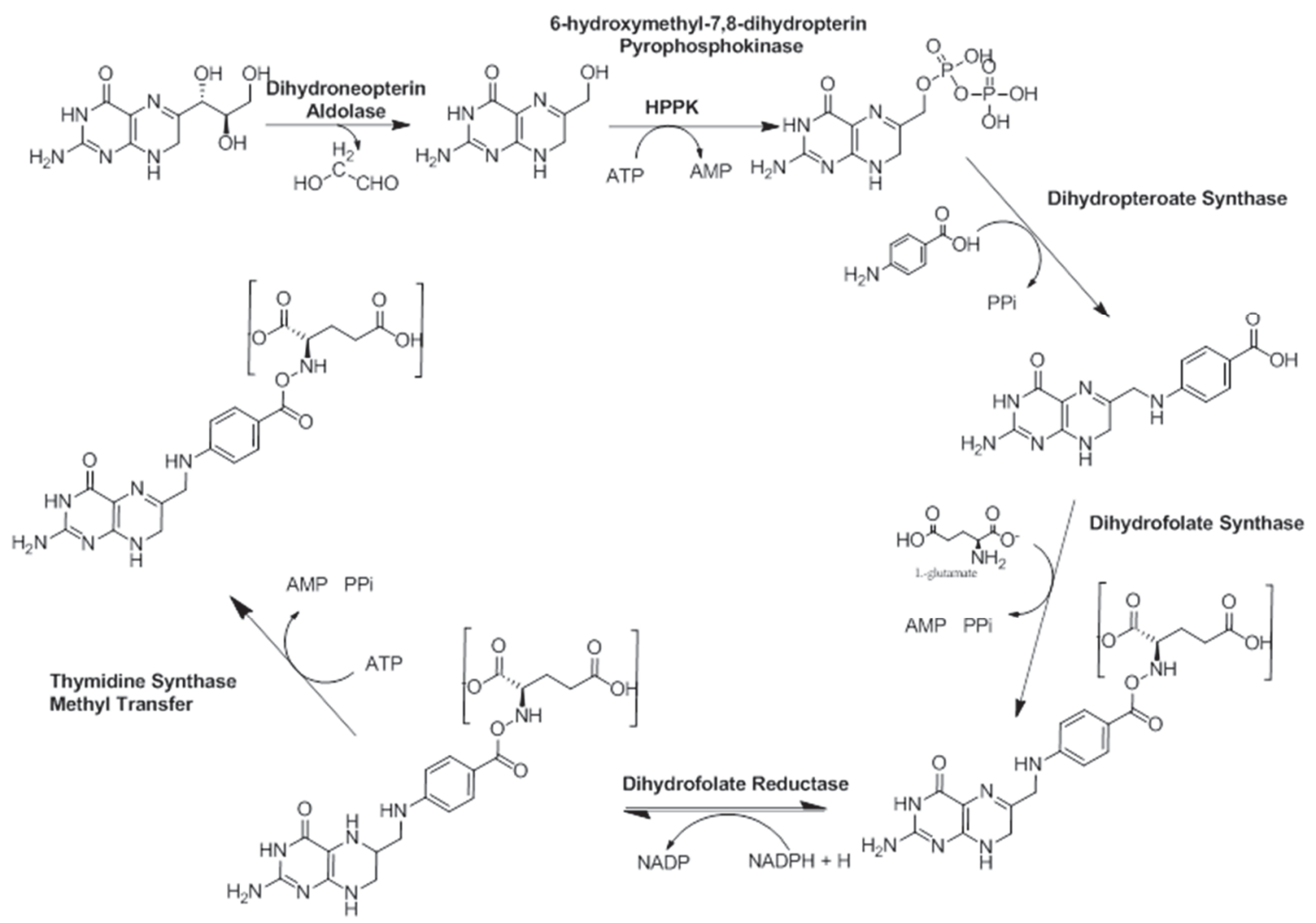

Figure 3-1. Folate biosynthesis pathway. 


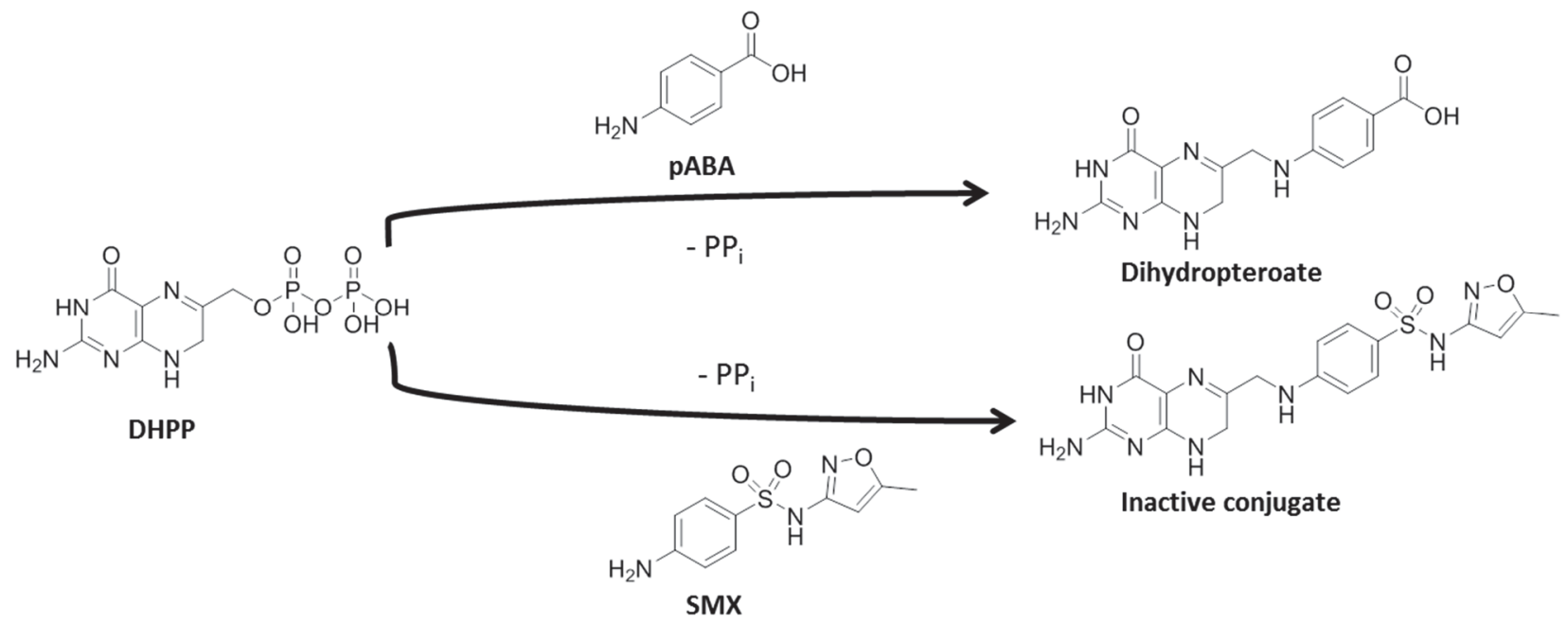

Figure 3-2. Illustration of SMX mimicking of pABA and the covalent modification of the metabolic precursor DHPP 
inhibitors with fragment screening to identify new scaffolds (ii) Drug repurposing screens for PCP using known DHPS inhibitors St Jude and malaria actives collection. Work in this chapter reflects multi-disciplinary efforts that broadened my experiences beyond just medicinal chemistry. The first project introduced me to structural biology and the power that structure guided design can bring to a project. The second allowed me a chance to gain experience with how running and prioritizing a screening workflow goes, invaluable experience for drug discovery projects. We worked in close collaboration with the White lab, who have provided key structural biology and biochemical expertise. Data and conclusions presented herin have been in part taken from our prior joint publications [229].

\section{Structure Guided Design of HPPK Inhibitors}

HPPK is a small $(\sim 18 \mathrm{kDa})$ highly conserved enzyme with an $\alpha \beta$ fold that catalyzes the transfer of pyrophosphate from ATP to 6-hydroxymethyl-7,8-dihydropterin (DHP) to form 6-hydroxymethyl-7,8-dihydropterin-pyrophosphate (DHPPP), one of the two substrates of DHPS. DHP binds within a ridged pocket with the pterin ring $\pi$-stacked between two conserved aromatic residues, the triphosphate is coordinated by two essential $\mathrm{Mg}^{2+}$ ions that form a very anionic region and the adenosine ring fits into a conserved cleft. HPPK uses an ordered enzyme mechanism in which the ATP cleft is first occupied, followed by the binding of DHP. Similar to DHPS [7], HPPK uses stabilizing loop conformational changes to assemble the complete active site and the DHP binding pocket [230-233]. As such pterin-pocket binders can only bind in the presence of ATP, an important distinction going forward.

We recently have shown that DHPS from the pathogen Francisella tularensis is a bifunctional enzyme and fused to 6-hydroxymethyl-7,8-dihydropterin pyrophosphokinase (HPPK) that catalyzes the previous step in the pathway [234]. We also showed that a known DHPS pterin-pocket inhibitor (1, Figure 3-3) engages the HPPK pterin pocket, even though there is no structural similarity between the pockets. Despite its high conservation and pivotal role in folate synthesis, there have been relatively few drug discovery efforts against HPPK [235-237]. This is somewhat surprising because there are a plethora of solved HPPK crystal structures available [234, 235, 238-241] and its catalytic mechanism is well understood [230, 231, 236, 237, 242-246]. This untapped potential has been noted, and there has recently been renewed interest in HPPK as an antimicrobial drug target [247-251]. The initial study yielded a number of compounds with significant binding affinity and inhibitory properties as 'hit' molecules, and using a structure-based approach, we have synthesized analog compounds and derived an initial SAR pattern. Based on these data, we then performed a similarity search of the NCI compound database for inhibitory pterin-like fragments, and identified and structurally characterized several potent scaffolds. As part of our lab's new initiative towards fragment based drug discovery, we have also performed thermal shift assays on our inhouse fragment set with SPR validation to identify more diverse chemical matter. HPPK provides us a potential novel mechanism of action for antibiotic development for drug 

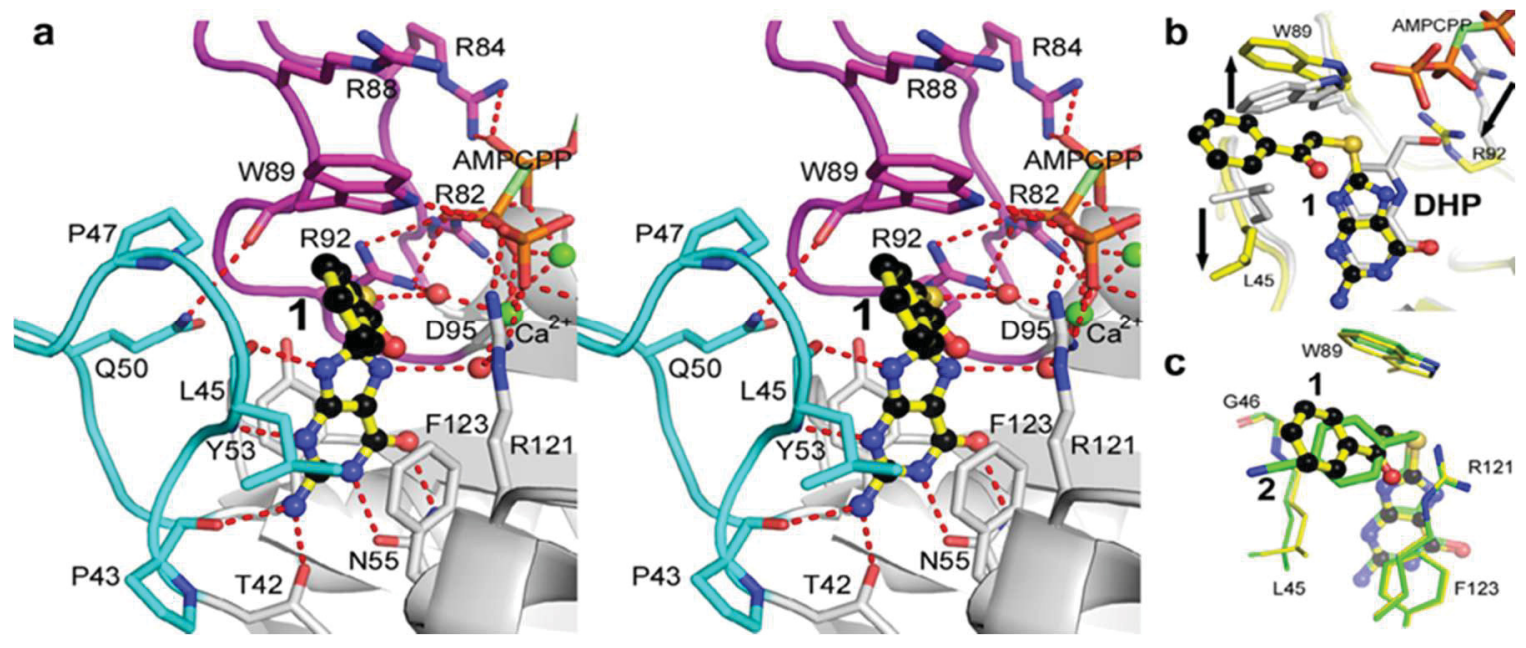

Figure 3-3. Crystal structure of 1 and 2 bound at the active site of HPPK

Depicts the loop2 (cyan) and loop3 (magenta) stabilizing interactions. Hydrogen bonding shown in red dashed lines, conserved waters shown as red spheres and phosphates shown in orange/green. A. Compound $\mathbf{1}$ bound B. 1 crystal structure overlayed with DHP structure highlighting the W89 shift to accommodate the 8-thioguanine substituent. C. Overlay of $\mathbf{1}$ and $\mathbf{2}$ showing the deeper reach of $\mathbf{1}$ and proximity to R121.

Reprinted with permission from Elsevier Science. Yun, M et al (2014). "The Identification, Analysis and Structure-Based Development of Novel Inhibitors of 6Hydroxymethyl-7,8-Dihydropterin Pyrophosphokinase.” Biorg. Med, Chem. 22 (1):215765 
resistant bacteria as there are no agents in the clinic targeting this conserved and essential drug target.

\section{Results}

During the course of our drug discovery studies on DHPS, our project team has generated a library of 230 pterin pocket targeted binding molecules. These compounds were designed to mimic the interactions with neighboring pyrophosphates and the neighboring pABA site of DHPS substrate's (DHPP) pterin core, and some minor substructure modifications. With the use of an endpoint HPPK assay that monitors unprocessed ATP substrate as a direct readout of inhibition, we screened these compounds against E. coli HPPK (EcHPPK). The screen revealed 2 compounds that significantly inhibit HPPK at $250 \mu \mathrm{M}$ (compounds 1 and 2, Table 3-1). To characterize the binding of $\mathbf{1}$ and $\mathbf{2}$ to EcHPPK in more detail, we used SPR to measure their binding characteristics. EcHPPK was immobilized on a sensor chip, and binding was measured in the absence and presence of $2 \mu \mathrm{M}$ of the non-hydrolysable ATP analog AMPCPP. As noted previously, compounds showed no appreciable binding in the absence of AMPCPP but robust binding in the presence of AMPCPP. The assembly of the pterin-binding pocket depends on ATP-dependent conformational changes in the three active site loops [230], and the SPR data are therefore consistent with 1 and $\mathbf{2}$ both engaging the pterin pocket (Table 3-1). We used this SPR protocol to measure the $K_{\mathrm{D}}$ of DHP (Table 3-1), and this confirmed that $\mathbf{1}$ and $\mathbf{2}$ are weak initial hit molecules in need of medicinal chemistry optimization to effectively compete with the HPPK substrate. It should be notes that our measured $K_{\mathrm{D}}$ for DHP is significantly higher than that previously reported [233] but we attribute this discrepancy to using a much lower concentration of AMPCPP used in our experiment $(2 \mu \mathrm{M}$ vs $100 \mu \mathrm{M})$ which may have an effect on the pocket stability. Isothermal titration calorimetry experiments support this explanation.

Compounds $\mathbf{1}$ and $\mathbf{2}$ are both substituted 8-thioguanines, and their ability to mediate the inhibition of EcHPPK is consistent with similar studies on Staphylococcus aureus HPPK (SaHPPK) [247]. This study was structural characterizations using X-ray crystallography on the unsubstituted 8-thioguanine, and determined that that similar $\pi$ stacking occured between the two conserved aromatic side chains and the oxygen and nitrogen atoms on the 8-thioguanine engaging in hydrogen bonding interactions similar to those of DHP. However, compounds $\mathbf{1}$ and $\mathbf{2}$ appear to be more potent than either 8thioguanine itself or derivatives that were recently reported [250]. Overall, both structures are very similar to that of the previously reported HPPK AMPCPP DHP substrate ternary complexes; Figure 3-3A shows a detailed stereo view of the compound 1 ternary complex. Much like the unsubstitued 8-thioguanine, this scaffold occupies the pterin pocket in much the same way as the pterin ring of DHP. It is sandwiched between Tyr53 and Phe123, and ring oxygen and nitrogen atoms engage in specific hydrogen bonding interactions with the side chains of Thr42 and Asn55, the main chains of Pro43 and Leu45, and a number of structured water molecules. Loop2 and loop3 both adopt ordered structures to facilitate these interactions that closely resemble their conformations in the substrate ternary complex. The DHP ternary complex suggests that substituents at 
Table 3-1. All HPPK compounds with in vitro data

$\begin{array}{llllll}\text { Compound } & \text { Solubility } \\ (\boldsymbol{\mu g} / \mathbf{m L})\end{array}$


the 8-thio position would not be tolerated because of steric clashes with the side chains of Leu45 and Trp89. However, the various 8-thio substitutions create a new cryptic binding pocket by an induced fit mechanism in which these residues rotate to generate the required space for the linked phenyl rings (Figure. 3-3B). These structural conformational changes held true for compound $\mathbf{2}$ which looks virtually identical apart from a slightly different orientation of the substituent within the induced pocket, perhaps due to the shorter extension away from the core 8-thioguanine scaffold and the lack of a sp2 carbon in the linker (Figure 3-3C).

\section{SAR Analysis of 8-Substituted-8-Thioguanine Derivatives}

The enzyme end-point assay and the SPR data both suggest that $\mathbf{1}$ is a marginally better inhibitor of EcHPPK than 2 (Table 3-1). Although the crystal structures are very similar, the source of this potency appears to be a key interaction in $\mathbf{1}$ that involves the carbonyl group on the linker between the phenyl and 8-thioguanine moieties (Figure. 33A). Specifically, the carbonyl group stacks onto the guanidinium group of Arg121 that, in turn, forms a stabilizing salt bridge interaction with the c-phosphate of AMPCPP. The carbonyl group is not present in $\mathbf{2}$, and the terminal phenyl group moves further into the pocket to partially stack with Arg121 (Figure. 3-3C). However, this movement puts additional strain on Leu45 (Figure. 3-3C), and appears to result in a more hindered binding conformation within the cryptic pocket.

Having established the importance of the carbonyl group within 1, we synthesized and tested the activities of a number of variants with different substitutions on the phenyl ring. Table 3-1 shows the 7 derivatives (compounds 3-9) with comparable activities to 1 and 2 , and 7 compounds with significantly reduced activities (compounds 11-17). Initial structure activity analysis of the latter compounds reveals some seemingly prohibitive substitutions. Extension of the phenyl substituent (11) using a Williamson ether synthesis to attach a morpholine ring resulted in an abolition of activity when compared to its $p$ hydroxyl parent (4). Loss of the aromaticity off the end of the chain with and without the carbonyl resulted in a deletion of inhibition (12 and 17) when compared to any of 3-9. A slightly larger methoxy substitution at the ortho position of the phenyl ring (13) saw a dramatic reduction in activity when compared to the methyl at that position (9). Finally we attempted to introduce chiralilty by introducing a methyl in the linker system (14 and 15), to break up any crystal packing that these relatively flat, polar molecules may be experiencing. There racemic mixtures were tested and found to be relatively weak binders compared to their corresponding linear partners.

It was difficult to distinguish the activities of the active inhibitors using this endpoint inhibition assay, and we therefore determined their $K_{\mathrm{D}}$ values using SPR and also measured their $\mathrm{IC}_{50}$ values by conducting the enzyme assay at increasing concentrations of compound between 0 and $500 \mu \mathrm{M}$. The SPR data and the $\mathrm{IC}_{50}$ measurements are shown as $K_{D}(\mu \mathrm{M})$ and $\mathrm{IC}_{50}(\mu \mathrm{M})$ respectively in Table 3-1. Overall, small substitutions at the para and meta positions $(\mathbf{3}, \mathbf{4}, \mathbf{5}, \mathbf{7}$ and $\mathbf{8})$ are preferred over larger para substitutions (6) and ortho substitutions (9). To investigate this pattern more 
systematically, the crystal structures of the ternary complexes with $\mathbf{3}, \mathbf{7}, \mathbf{8}$ and $\mathbf{9}$ were determined (Figure 3-4B-E).

All four ternary complex structures are virtually identical to that of $\mathbf{1}$, and the only significant differences occur in the revealed cryptic pocket (Figure 3-4B-E). In the 3 and 7 ternary complexes (Figure 3-4B and C), the para-substituents extend directly away from the pocket, and this appears to 'push' the phenyl group deeper into the interior of the pocket by $1 \AA$ compared to $\mathbf{1}$. This results in a more favorable stacking interaction between the planar carbonyl and the guanidinium group of Arg121, and may explain why the para substitutions generally display superior activities. The 8 structure (Figure 3-4D) shows that the meta-substituent has little effect on the binding conformation of most of the structure, and is able to form a favorable van der Waals interaction with Leu45 explaining its modest increase in affinity. Finally, the 9 structure (Figure 3-4E) reveals that the ortho-methyl group is directly adjacent to Leu45 which is forced to rotate further away compared to the other structures. This explains the slightly diminished activity of $\mathbf{9}$, and the much lower activity of $\mathbf{1 3}$ in which the ortho-substituent is somewhat larger and would force Leu45 to an even more strained position.

\section{Fragement Studies on the HPPK Pterin-Binding Pocket}

We found that 8-thioguanine (10) is a very poor inhibitor of EcHPPK and shows minimal binding by SPR in the presence of AMPCPP (Table 3-1). This explains why, despite a number of attempts, we have failed to obtain a crystal structure of EcHPPK bound to 10. It was previously predicted that the sulfur atom in $\mathbf{1 0}$ would form a van der Waals interaction with Trp89 [247], and our structures of $\mathbf{1 , 2}, \mathbf{3}, \mathbf{7}, \mathbf{8}$ and $\mathbf{9}$ have not only confirmed this but revealed the potential for a steric clash (Figure 3-5). It appears that our inhibitory compounds that contain this scaffold are only able to bind because the substituents 'pull' the scaffold away from Trp89 and also rotate the side chain, thereby relieving the steric clash. This in turn suggests that $\mathbf{1 0}$ is actually a suboptimal scaffold that compromises the binding of all the compounds that contain it. Given this limitation, as well as a derth of any significant whole cell antibacterial activity we decided to search for alternative scaffolds for optimization. To do this we launched a multifaceted fragment based approach; first to identify novel pterin-like fragments around the 2D structure of a more potent 6-thioguanine, and next an unbiased screening thermal denature screen of fragments in the presence and absence of AMPCPP.

We first examined 6-thioguanine (18) in which the sulfur atom is moved to a position on the scaffold that would not interact with Trp89. Although solubility issues prevented the measurement of a meaningful $\mathrm{IC}_{50}$ value, $\mathbf{1 8}$ has demonstrated to be a far superior inhibitory scaffold than 8-thioguanine based on percent inhibition (Table 3-2) and has now been made into our control compound for all biophysical screening approaches. This superiority was confirmed by SPR that revealed a low $\mu \mathrm{M} K_{\mathrm{D}}$ value and showed that it binds to the pterin pocket in complex with AMPCPP. The crystal structure of the ternary complex (Figure 3-6A) confirms that 18 engages the pterin pocket in the same fashion as the 8-thioguanine scaffold, but without the steric clash with Trp89. 18 is 

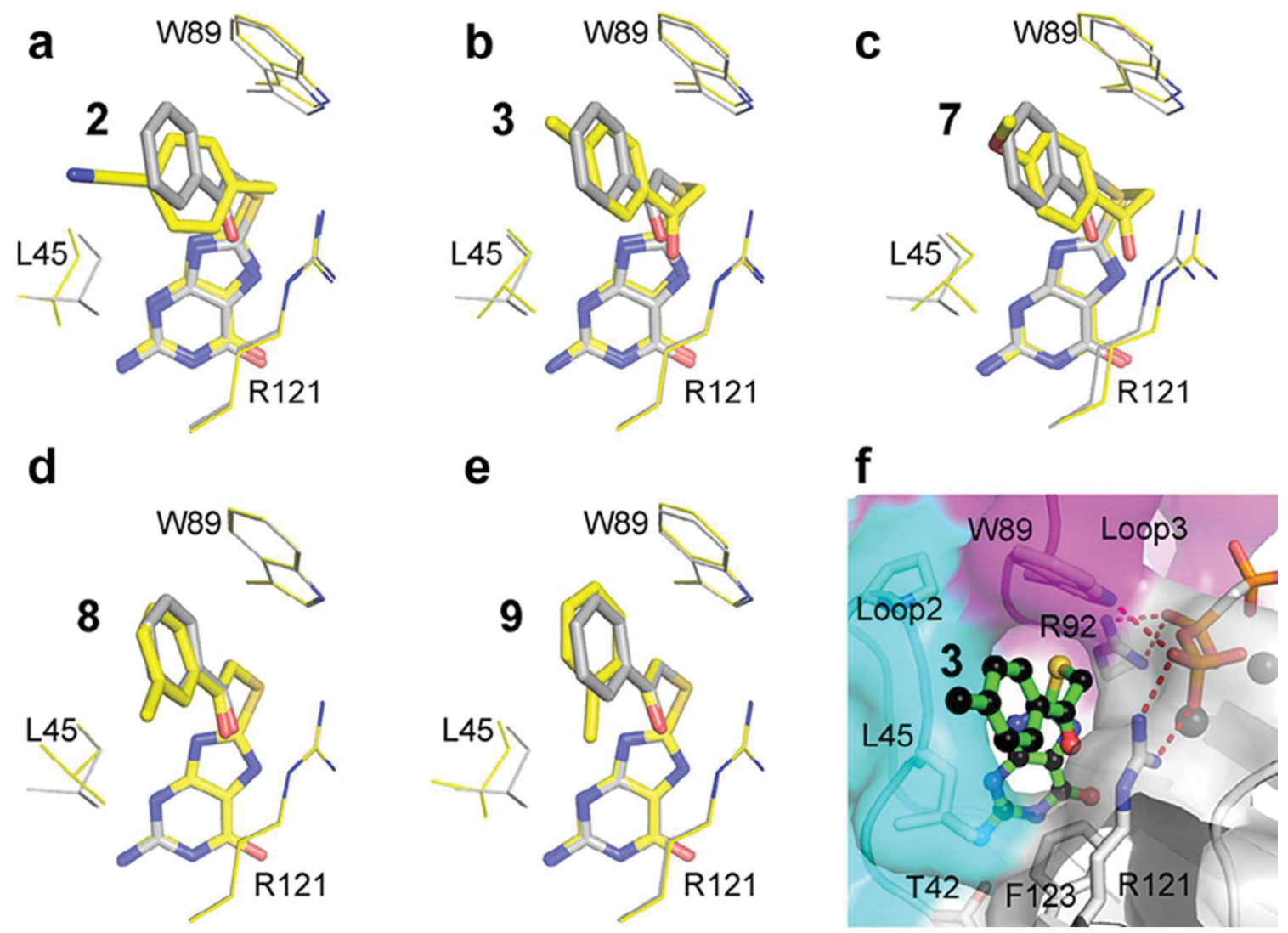

Figure 3-4. A-E. Abridged crystal structures of HPPK and inhibitors

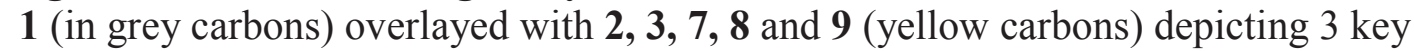
residues. F. 3 in a surface representation of the active site highlighting the induceded cryptic pocket formed to accommodate the 8-substituted thioguanines.

Reprinted with permission from Elsevier Science. Yun, M et al (2014). "The Identification, Analysis and Structure-Based Development of Novel Inhibitors of 6Hydroxymethyl-7,8-Dihydropterin Pyrophosphokinase.” Biorg. Med, Chem. 22(1):215765 


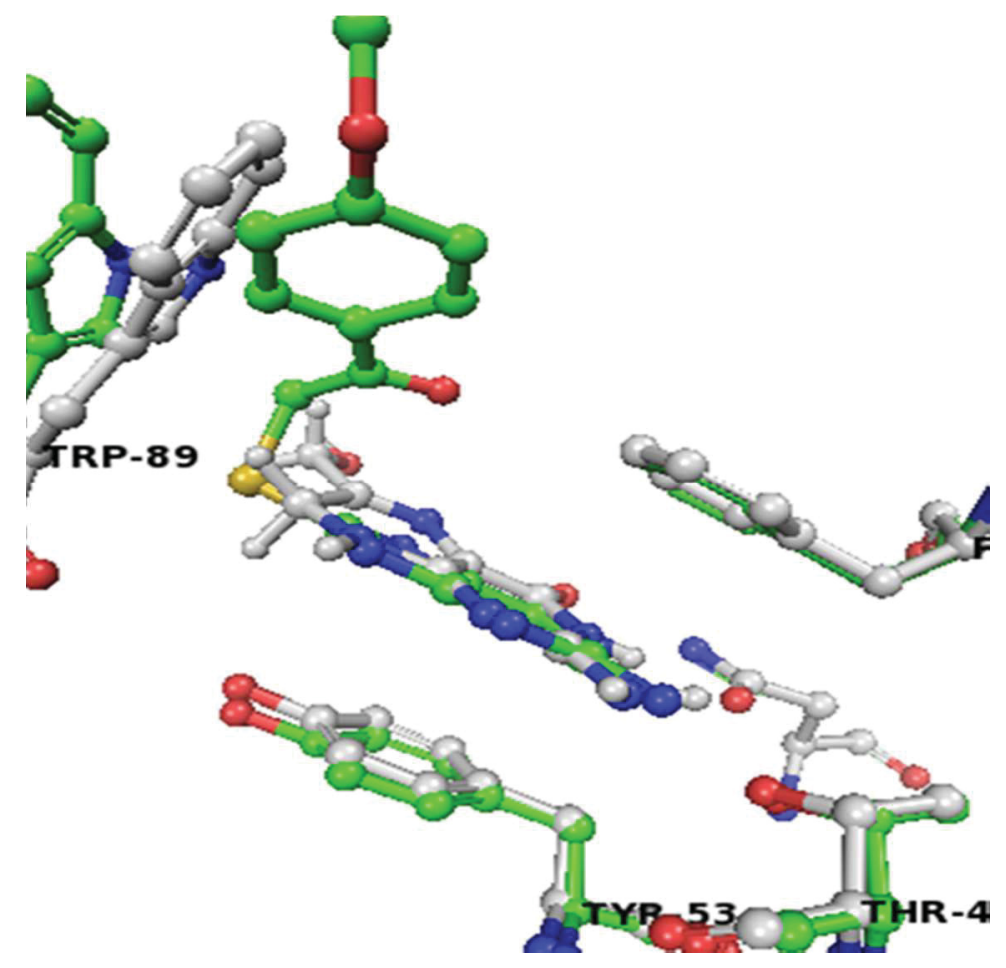

Figure 3-5. Crystal structure of HPPK ternary complex with compound 7 7 (green) overlaid endogenous DHP (gray), showing the steric interaction with TRP89. 
Table 3-2. HPPK inhibition data from 2D fragment screen

\begin{tabular}{cccccc}
\hline Compound & Structure & \% Inhibition $(\mathbf{2 5 0} \boldsymbol{\mu M})$ & $\mathbf{K}_{\mathbf{D}}(\boldsymbol{\mu} \mathbf{M})$ & PEI \\
\hline $\mathbf{1 8}$ & 51 & $18 \pm 1$ & 3.05 \\
$\mathbf{1 9}$ & 48 & $38 \pm 3$ & 3.18 \\
$\mathbf{2 1}$ & 49 & & & \\
\end{tabular}



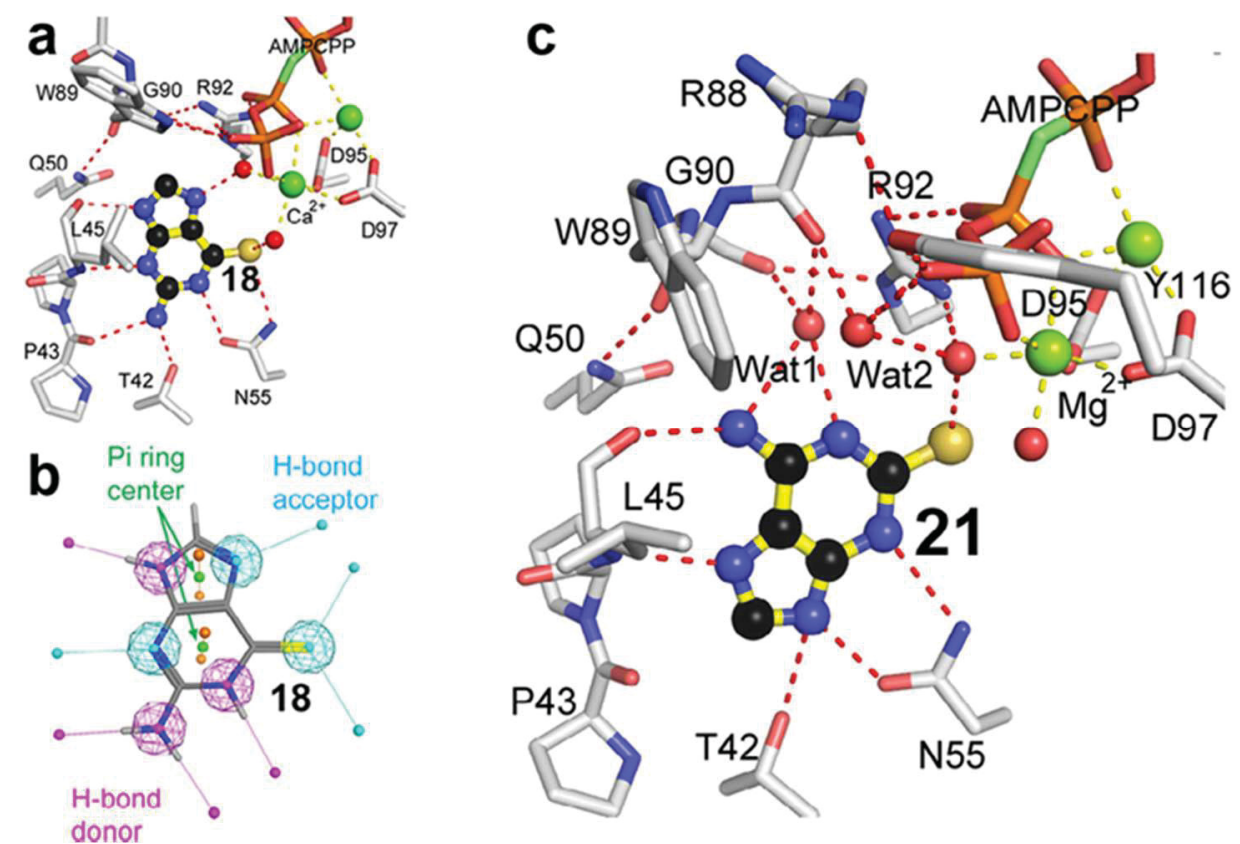

Figure 3-6. Two dimensional fragment screen

Crystal structure of HPPK ternary complex with compound fragments $\mathbf{1 8}(\mathbf{A})$ and $\mathbf{2 1}(\mathbf{C})$. B. The 2D pharmacophore used to screen the fragment database.

Reprinted with permission from Elsevier Science. Yun, M et al (2014). "The Identification, Analysis and Structure-Based Development of Novel Inhibitors of 6Hydroxymethyl-7,8-Dihydropterin Pyrophosphokinase.” Biorg. Med, Chem. 22(1):215765 
a low molecular weight efficient HPPK binder that can be considered a fragment 'hit', and its ligand efficiency can be monitored using the 'Percent Efficiency Index' (PEI) metric [252]. This value for $\mathbf{1 8}$ confirms the suitability of the molecule for future elaboration. Given the highly polar nature of fragments expected to bind at this particular site, pure ligand efficiency is more a more practical way of analyzing hits for this particular series.

Next, applying best practices from other fragment-based drug discovery programs $[253,254]$, we decided to search for fragments similar to 18 's scaffold to identify the best starting templates for further elaboration. We did this by conducting a $2 \mathrm{D}$ similarity search for new fragments with high Tanimoto similarity to $\mathbf{1 8}$ against the NCI database of compounds and applying a molecular weight cutoff of 300 Daltons. 25 fragments were shortlisted, and 17 were able to be obtained from the NCI and verified to be $>95 \%$ pure using HPLC analysis. Docking simulations using AutoDock showed that they all have the potential to bind within the pterin-binding pocket in a similar manner to $\mathbf{1 8}$ (Figure 3-6B). When tested, three of these fragments, 19, 20 and 21 (Table 3-2), showed inhibitory activities around $50 \%$ with PEI values similar to $\mathbf{1 8}$. All three are dihydropurine fragments that also showed AMPCPP-dependent binding by SPR with $K_{D}$ values comparable to 18 (Table 3-2). These fragments suffered from limited solubility that did not allow an accurate measurement of their $\mathrm{IC}_{50}$ values. 19 and $\mathbf{2 0}$ both resemble 8-thioguanine, but with the 8-position sulfur atom replaced with a hydrogen atom and a methyl group, respectively. Their improved potencies from $\mathbf{1 0}$ and our abilities obtain a crystal structure confirm our theory that the bulky sulfur atom in 8-thioguanine leads to a steric clash with Trp89 that disfavors binding. Compound $\mathbf{2 1}$ is unique from any of the other scaffolds but still binds to the pterin site. The sulfur atom engages the same space as that of $\mathbf{1 8}$ adjacent to Asn55, but slightly different pocket conformations allows a slightly higher binding affinity.

Finally, a full biophysical fragment screen was conducted to establish alternative binders that may escape some of the PK issues that have plagued our scaffolds so far. We conducted a thermal shift assay first, a high throughput means of detecting ligands that stabilize a protein complex which is interpreted as binding affinity. Protein is heated in the presence of a fluorescent dye until a temperature is reached which destabilizes the protein and reveals the inner hydrophobic regions which react with the dye and produce a readable signal. In the presence of a ligand, the free energy of binding that allows a molecule to bind also stabilizes the protein. In theory, the higher the affinity of the ligand, the higher the degree of stabilization for the complex and will result in a corresponding "shift" in the melting temperature. We screened our 4,500 fragments in the presence and absence of AMPCPP to sample fragments that would be expected to bind in the pterin binding pocket. Pterin specific hit molecules were rescreened on the SPR to confirm binding and determine affinity. To date we have produced three more potential scaffolds that seem to bind with weaker affinity, but offer more in the way of chemical diversity (Figure 3-7). These fragments are being re-procured to properly assess accurate binding affinity from freshly prepared pure stocks and for submission for X-ray crystallography. We are still deconvoluting promiscuous binders to determine if there may be another 

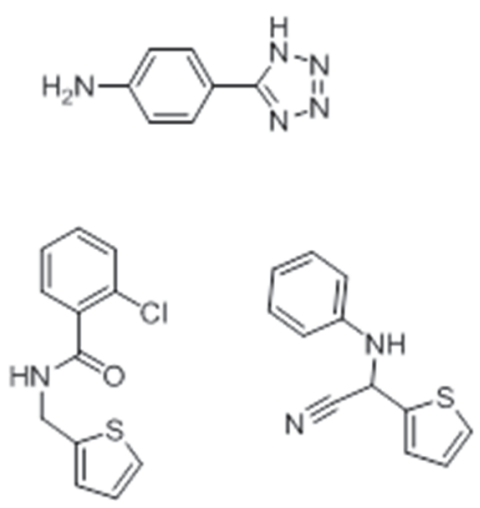

Figure 3-7. Biophysical fragment screen

Weak affinity fragments from biophysical screen. All three bound tighter in the presence of $\mathrm{PP}_{\mathrm{i}}$ which implies they are binding at the pterin binding pocket. 
binding site that inhibitors could occupy regardless of ATP and its corresponding loop conformational change.

\section{Discussion}

The bacterial enzymes HPPK and DHPS catalyze adjacent steps in the folate biosynthetic pathway, and they each have very specific pockets that engage their pterin substrates. Although the two pockets have clearly evolved independently and have very different architectures, our recent demonstration that a small molecule can bind both pockets [234] suggests that generating more pterin-like scaffolds could be useful in identifying potent inhibitors of both enzymes. The successful identification of HPPK inhibitors from our library of molecules designed to engage the DHPS pterin-binding pocket supports this proposal. We identified and discussed three classes of HPPK inhibitors, one containing an 8-thioguanine core, fragments with pterin-like bicyclic scaffolds, and several unique fragment molecules that engage the pterin pocket in ways that have been characterized by X-ray crystallography and SPR. These new fragment molecules can now be elaborated and optimized as HPPK inhibitors, and we will also investigate their potential for being developed as novel inhibitors of DHPS.

Independent studies have previously identified 8-thioguanine as an HPPK inhibitor suitable for development [247], and preliminary attempts to do this were recently reported [250]. These studies, using HPPK from S. aureus, showed that 8thioguanine can bind in the absence of nucleotide, but our SPR data show that binding is minimal both in the absence and presence of nucleotide. In the published structure, 8 thioguanine displaces the Trp89 of the flexible loop3 which seems to disfavor crystallization. Our structures have confirmed the prediction [247] that the sulfur atom is directly adjacent to Trp89 in the ternary complex and causes a steric interaction. However, the binding of our 8-thioguanine derivatives only appears possible because the 8-position substituents displace Trp89 and create the necessary space in the form of a cryptic pocket. Even more recent work than what has been discussed has emerged that delves into the structural basis of these inhibitors and their selectivity for HPPK [251].

Our future goals will be to use these preliminary data to develop more potent inhibitors of HPPK with whole cell activity using structure-based approaches. Our current results have suggested a number of avenues to achieve this that will be pursued. First, we have identified a number of novel and quite potent pterin pocket binding scaffolds, and each of these will be elaborated into mini libraries for further screening. Crystal structures of ternary complexes containing two of these fragment scaffolds have revealed unanticipated plasticity in the HPPK pterin pocket. Second, our analyses of the 8 -thioguanine derivatives have provided key information on how these scaffolds can be elaborated to take advantage of a pocket adjacent to the active site that is induced upon binding. This pocket has in fact been previously identified from earlier structural studies of an HPPK inhibitory molecule that contains the pterin scaffold [236]. Our initial analyses have revealed several SAR features of substituents that improve binding within this pocket, and additional optimization will be pursued. Finally, the nucleoside and 
triphosphate binding sites are potentially rich unexploited locales for further optimization of our evolving molecules. The build-out of our inhibitors into these sites offers the possibility of generating tight-binding nucleotide-independent inhibitors. Encouragingly, similar strategies involving the synthesis of bisubstrate analogs (pterin-nucleotide adducts) have already demonstrated the feasibility of this approach for this target [237, 249], and have provided important clues as to how to proceed.

Antimicrobial anti-folate therapeutics that contain a combination of sulfa drugs (DHPS) and dihydrofolate reductase inhibitors have proven to be very effective [255], and HPPK/DHPS pterin-based inhibitors offer the possibility of alternate combination therapies. The high conservation of the HPPK and DHPS pterin pockets in all microorganisms should mean that these therapies have broad spectrum potential that are less prone to resistance mutations. Not only would agents targeting HPPK be incredibly useful in sulfonamide resistant bacterial populations; these agents would help patients with sulfa-allergies and in patient populations with high incidence of sulfa allergies such as those with HIV undergoing prophylaxis to prevent pneumocystis pneumonia [228]. To obtain such therapeutics, it is clear that these compounds must be further optimized to enhance target affinity and microbial penetration, and to maximize exposure in the host. We believe that the structural and biochemical data presented in this study have provided an excellent basis to enable such efforts to move forward rapidly.

\section{Repurposing for Pneumocystis Pneumonia}

Pneumocystis pneumonia (PCP) first emerged in the United States as a serious infection risk in the late 1970'sin patients undergoing intense chemotherapy or suffering from severe immune suppression. PCP is an opportunistic infection of the lungs that relies on the host immune system to be compromised (CD4+ T-lymphocyte cell count $<$ 200 per $\mathrm{mm}^{3}\left(200 \times 10^{6}\right.$ per L)). The causative pathogen in humans is Pnuemocystis jirovecii, previously known as Pnuemocysistis carinii (this name denotes the murine form still), a yeast-like fungus that shares some ancestral lineage with protozoan parasites Trypanosoma cruzi and Plasmodium falciparum [256]. The primary prophylaxis of PCP was developed at St Jude by Dr. Walter Hughes, that being SMX:TMP. In contrast to most bacterial infections, SMX is the more potent in this combination as TMP has low affinity for the Pneumocystis isoform of the DHFR enzyme. Inhibitors have been designed to target the $P$. carinii DHFR (pcDHFR) in mice with limited clinical success [257]. However in this vein, researchers have shown that the pcDHFR can be preferentially selected in TMP analogs in comparison to the human form [258]. This is due to slight differences in a few key residues, similar to the difference in selectivity between TMP and the human DHFR inhibitor methotrexate. This section will focus on two drug repurposing screens that take advantage of the intrinsic susceptibility to sulfa drugs and the relationship pneumocystis have with protozoan parasites. 


\section{Introduction: Repurposing for New Leads Against Pneumocystis}

Drug repurposing is rapidly becoming more popular in antibiotic drug discovery [259]. The cost of developing novel chemical entities from initial discovery into the clinic is a major deterrent for large pharmaceutical companies to enter into antibiotic drug discovery. This is especially problematic with unknown properties associated with attempting to drug a new target. With federal safety regulations as high as they have ever been, long term toxicity is equally as important as efficacy in many cases. Drug repurposing of either FDA approved drugs or classes of drugs that have extensive preclinical safety data is a fantastic way to bias "hit" compounds towards having a high safety profile. As previously discussed, this is a popular area in TB drug discovery with the $\beta$-lactams, oxazolidinones and fluoroquinolones all being repurposed agents that have had high degrees of success. There are many examples of non-antibiotic drugs that have been found to have activity in both gram-positive and negative bacteria [259], that have invigorated researchers to take up this approach in earnest.

Neglected disease drug discovery has leaned heavily on drug repurposing to generate new lead molecules to be optimized. These fields are not typically high revenue generating areas, so large companies are hesitant to pour resources into a space with limited return on investment. In addition to the TB drugs discussed before, many preclinical anti-malaria drugs have been identified off of so called "piggy-back" discovery [260]. This is where investigators pick a drug target being investigated in a different organism that has a high degree of similarity to a known target in the parasite. The cited example is repurposing anti-cancer histone deacetylase inhibitors and osteoporosis cysteine protease inhibitors for anti-parasitic activity. Once leads are identified, SAR studies are generated to increase potency and disease specificity to develop highly specific antagonists. Pneumocystis is notoriously difficult to grow in culture, and this makes screening very difficult. This is likely why there is not a lot of screening data available currently and why there is a serious lack of therapeutic alternatives in the cases of drug resistance. With the help of Dr. Melanie Cushion at the University of Cincinnati College of Medicine we have been able to screen our libraries of compounds in an ex vivo model from rat lung cultures, a system outlined in the results and methods.

DHPS inhibitors (ie sulfadoxine and sulfamethoxypyridazine) have been shown to be useful agents in the treatment of malaria, especially in combination with pyrimethamine, a DHFR inhibitor. These agents have a particular affinity for the $P$. falciparum isoforms of their respective enzymatic targets, hence their use over the more traditional SMX:TMP combination. At St Jude we have a large and data laden library of anti-malaria compounds generated by the Guy laboratory and available for screening in other projects. Many of these do not have known mechanisms of action, but it is likely that many would be inhibitors of this essential pathway. We have clustered and selected chemically diverse, potent and non-cytotoxic compounds from this library for screening against Pneumocystis. Eventually the hits we have produced will be screened for DHPS activity and then optimized for selectivity and potency. Our second repurposing screen is the re-examination of legacy compounds from our lab, inhibitors of the DHPS enzyme 
designed to be effective in sulfonamide resistant bacteria, to target Pneumocystis infections. These two diametrically opposed ways of screening highlight the advantages and disadvantages of targeted phenotypic screening.

\section{Results}

Using data from the previous HTS of St Jude library of 500,000+ compounds and malaria targeted compounds, we selected compounds with a high therapeutic index (TI) relative to their malaria $\mathrm{IC}_{50}$ and multiple mammalian cell line toxicity $\mathrm{IC}_{50} \mathrm{~s}$. The hypothesis here was that malaria actives may be a good source of novel antipneumocystis compounds, due to overlap in anti-folate efficacy. These compounds were clustered into 33 chemically diverse series, 16 of which are shown in Figure 3-8. These representative molecules were cherry picked and screened at high concentrations (100 $\mu \mathrm{g} / \mathrm{mL}$ ) against $P$. carinii. Cryopreserved and characterized $P$. carinii isolated from rat lung tissue was distributed into triplicate wells of 48-well plates with a final volume of $500 \mu \mathrm{l}$ and a final concentration of $5 \times 10^{7} \mathrm{nuclei} / \mathrm{ml}$. Controls and test compounds were added and incubated at $37{ }^{\circ} \mathrm{C}$. At 24,48 , and 72 hours, $10 \%$ of the well volume was removed and the ATP content was measured using Perkin Elmer ATP-liteM luciferinluciferase assay. The luminescence generated by the ATP content of the samples was measured by a BMG PolarStar optima spectrophotometer. A sample of each group was examined microscopically on the final assay day to rule out the presence of bacteria. Background luminescence was subtracted and triplicate well readings of duplicate assays were averaged. For each day's readings, \% reduction in ATP for all groups was calculated: experimental - experimental/vehicle control x100. Positive hits from the initial sceen ( $>50 \%$ inhibition) were subjected to rescreening in dose response to determine an $\mathrm{IC}_{50}$. These compounds were taken from a screening library that contained many analogs that are ready to be tested, another advantage of this approach as early SAR studies are available without synthesis. SMX was included in these screening run as a control and registered an $\mathrm{IC}_{50}$ of $13.39 \mu \mathrm{g} / \mathrm{mL}$.

From this bioactive screen we identified 3 potent scaffolds with $P$. carinii $\mathrm{IC}_{50}<$ $22 \mu \mathrm{g} / \mathrm{mL}$ including one with $\mathrm{ng} / \mathrm{mL}$ activity. More important, the TI for these hits was quite high in mammalian cell lines (Table 3-3). Being comparable or vastly superior ex vivo to SMX makes these scaffolds very intriguing and warrants a follow up investigation. Currently, in vivo toxicity trials have begun in a rat model as well as preliminary $\mathrm{PK}$ analysis to gauge formulations and administration before a full range efficacy study. These studies will further guide which scaffold to pursue, as many times the most potent compound could less favorable than one with a good in vivo PK or toxicity profile. Synthetic schemes have been postulated such as in Figure 3-9 to generate analogs around these scaffolds in an effort to improve drug like properties. Genetic sequencing of resistant mutants has begun in the hopes of identifying novel mechanism(s) of action that can be further studied.

Our second repurposing screen was multifaceted, centralized around screening DHPS inhibitors for $p$. carinii activity. First we focused on legacy compounds that had 

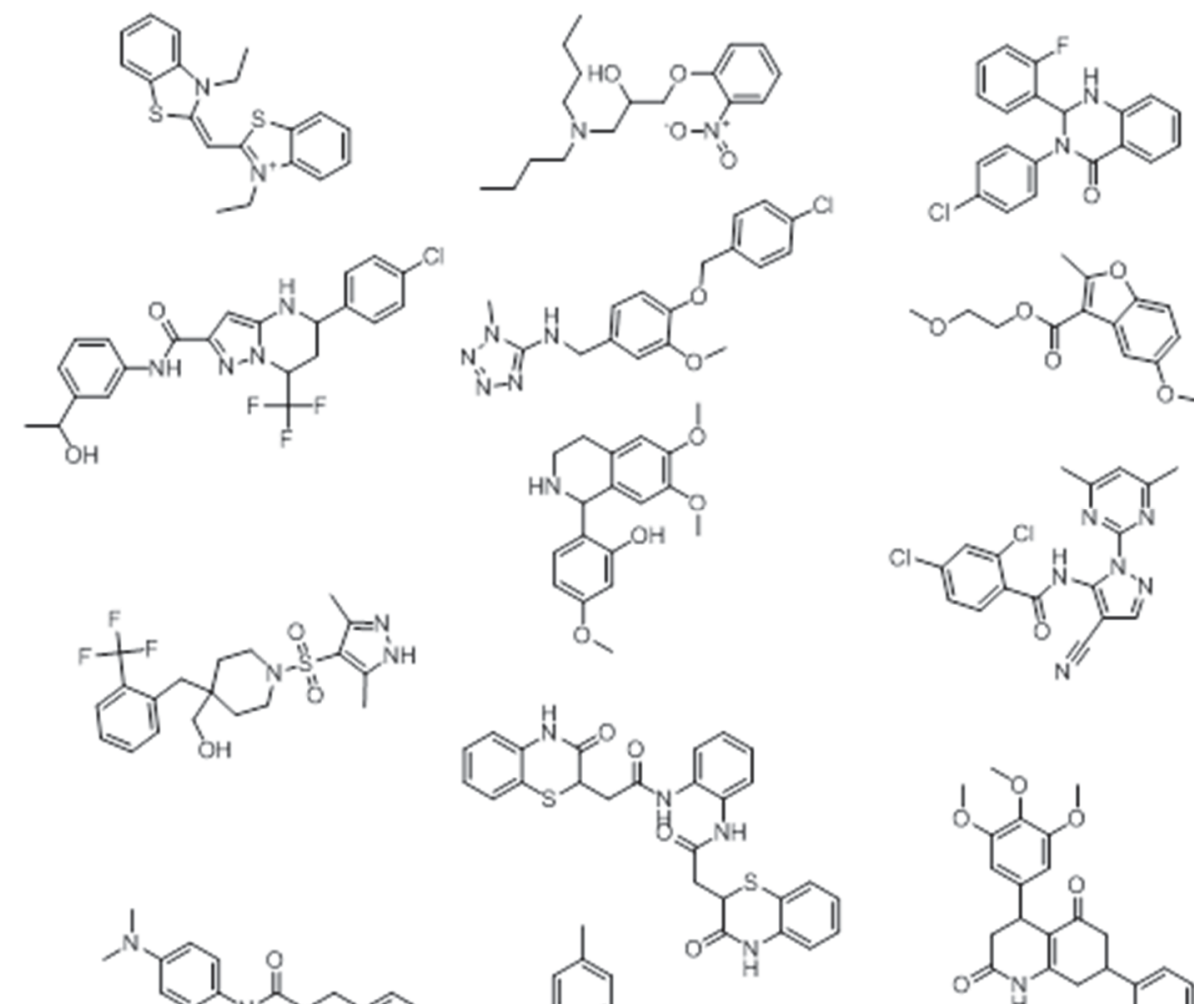<smiles>COCCOC(=O)c1c(C)oc2ccc(OC)cc12</smiles><smiles>Cc1cc(C)nc(-n2ncc(C#N)c2NC(=O)c2ccc(Cl)cc2Cl)n1</smiles><smiles>CN(C)c1ccc(NC(=O)CCc2ccccc2)cc1</smiles><smiles>Cc1ccc(-c2cc(=O)n(-c3nc(C)cc(C)n3)[nH]2)cc1</smiles><smiles>COc1cc(C2CC(=O)NC3=C2C(=O)CC(c2ccc(F)cc2)C3)cc(OC)c1OC</smiles><smiles>Cn1c(SCC(=O)Nc2cc(C(F)(F)F)ccc2Cl)nnc1-c1ccc(Br)cc1</smiles><smiles>O=C(O)c1ccc(CNCC(O)c2ccccc2)cc1</smiles>

Figure 3-8. 16 representative anti-malaria compounds of the 33 chemically diverse scaffolds selected for Pneumocystis screening 
Table 3-3. Malaria bioactive screen hits and their in vitro data

\begin{tabular}{|c|c|c|c|c|c|c|}
\hline Compound & Structure & $\begin{array}{l}\text { Pnuemocystis } \\
\text { IC }_{50}(\mu \mathrm{g} / \mathrm{mL})\end{array}$ & $\begin{array}{c}\text { MAR3D7 } \\
\text { IC } 50 \\
(\mu \mathrm{g} / \mathbf{m L})\end{array}$ & $\begin{array}{c}\text { HEPG2 } \\
\text { IC50 }_{50} \\
(\mu \mathrm{g} / \mathrm{mL}) / \\
\text { TI }\end{array}$ & $\begin{array}{c}\text { BJ IC50 } \\
(\mu g / m L) \\
/ ~ T I\end{array}$ & $\begin{array}{c}\text { HEK } \\
\text { IC } 50 \\
(\mu \mathrm{g} / \mathrm{mL}) \\
/ \mathrm{TI}\end{array}$ \\
\hline 550762 & & 0.0023 & 0.204 & $\begin{array}{l}5.524 / \\
2401.7\end{array}$ & $\begin{array}{l}53.975 / \\
23467.4\end{array}$ & $\begin{array}{l}9.514 / \\
4136.5\end{array}$ \\
\hline 551074 & & 4.89 & 0.074 & $\begin{array}{c}9.426 / \\
1.928\end{array}$ & $\begin{array}{l}437.729 \\
/ 89.515\end{array}$ & $68.129 /$ \\
\hline 14207 & & 21.97 & 0.009 & $\begin{array}{c}18.72 / \\
0.852\end{array}$ & 7023.060 & 3.868 \\
\hline
\end{tabular}



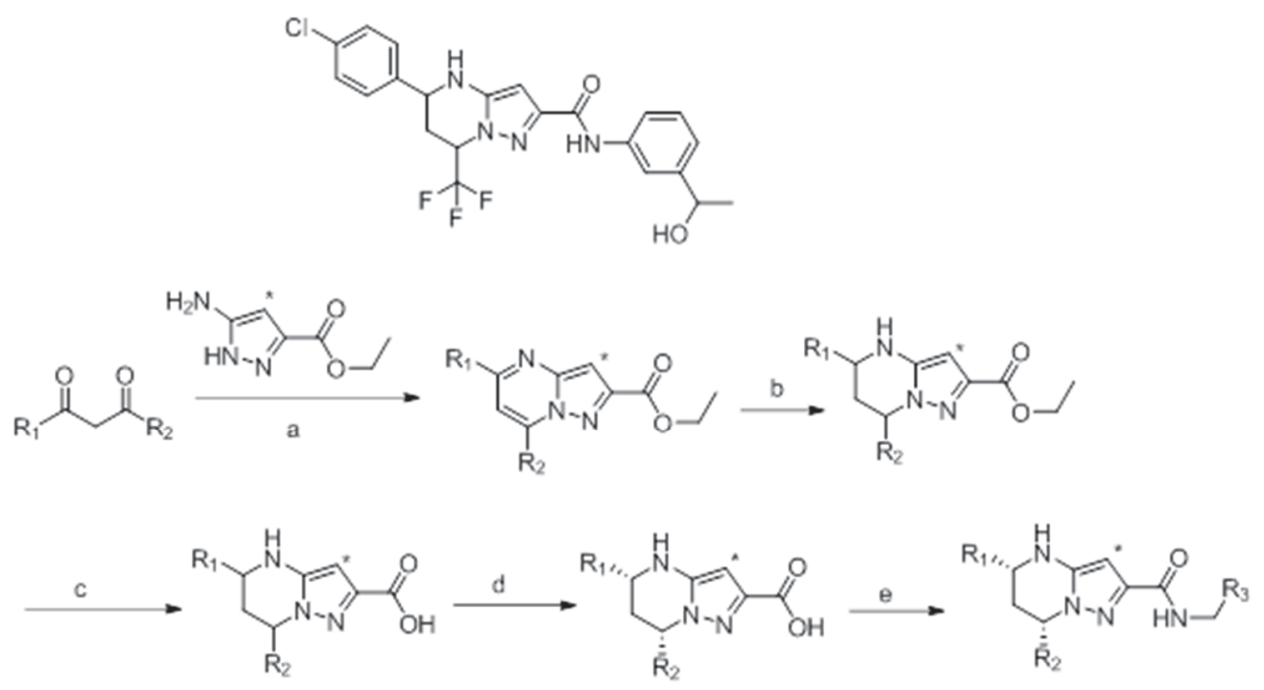

(a) $\mathrm{AcOH}$ at $110^{\circ} \mathrm{C}$; (b) $\mathrm{NaBH} 4$, EtOH at

room temperature; (c) $\mathrm{KOH}$, aq. EtOH at $60^{\circ} \mathrm{C}$; (d) preparative

chiral HPLC; (e) HATU, i-PrNEt2, DMF.

- Alternate ester position

Figure 3-9. Reaction scheme to generate analogs around one of the repurposing hits that demonstrates modularity and the ability to rapidly generate diverse analogs 
been previously synthesized as enzymatic inhibitors. This series in particular had potent DHPS IC 50 values, but were never able to achieve a high degree of whole cell activity; most likely due to poor solubility and cell penetration issues. They were designed to mimic the pterin portion of the DHPS substrate as well as the conjugated product of various approved sulfa drugs (Figure 3-10). Once we discovered some of the best inhibitors of Pneumocystis were the conjugate mimics, their corresponding sulfas were screened to see if we could increase potency with drugs that have better drug like properties. Most sulfa drugs are very soluble and have great oral bioavailability which would be attractive for developing new agents. Unfortunately these sulfas were by and large less efficacious than their conjugates (data not shown), but there are a few possible explanations in the discussion as to why this might be the case. The lone exceptions to this were SMX and the second line PCP drug dapsone, also a known DHPS inhibitor. To date we have identified 6 legacy compounds with comparable or superior activity to that of SMX (Table 3-4), not including 3 additional strong hits that need to be confirmed upon resynthesis for an accurate $\mathrm{IC}_{50}$. Just like with the bioactive screening, these top hits will be investigated in in vivo toxicity and PK studies to further delineate lead compounds to pursue.

\section{Discussion}

Due to the success of screening malaria actives against Pneumocystis so far, we are considering revisiting these anti-malaria molecules to find even more chemical diversity. This active subset of compounds is an excellent primary source of chemical matter to work on, especially with readily available analogs already generated, many with existing ADME and toxicity data. The results of genomic sequencing should lead to more micro- and molecular biology studies for target validation and resistance patterns, which in turn further aids our efforts to find new anti-pneumocystis drugs. Eliciting resistance mechanisms could help identify new drug targets in this opportunistic pathogen. It is very important for the field to be open and collaborative, there are countless compounds already produced and tested that are available for screening. This approach is powerful on any number of organisms and is a tremendous approach for both academics and industry alike to take advantage of. The kinase field alone has generated hundreds of thousands of non-specific kinase inhibitors. Many of these are ripe for this type of screening, finding those with higher affinity for a bacterial or other infectious pathogen that can be quickly optimized for even greater specificity. Such approaches have been successful in other neglected diseases [261], and the opportunity will grow with continued advances in screening and informatics handling of data.

Compound 550762 has an $\mathrm{IC}_{50}$ of $2.27 \mathrm{ng} / \mathrm{mL}$ (Table 3-3), which is over a thousand fold more potent than SMX. This degree of potency combined with a large TI $\left(\mu \mathrm{g} / \mathrm{mL} \mathrm{IC}_{50 \mathrm{~S}}\right.$ in multiple mammalian cell lines), is highly encouraging despite the presence of a possible indiscriminate covalent binding motif. In vivo toxicity studies will rapidly determine if this core scaffold is toxic, otherwise there is ample room for chemical modification on both symmetric ring systems to explore any possible SAR. Compound 551074 is also quite potent with an $\mathrm{IC}_{50}$ of $4.89 \mu / \mathrm{mL}$ (Table 3-3). This 


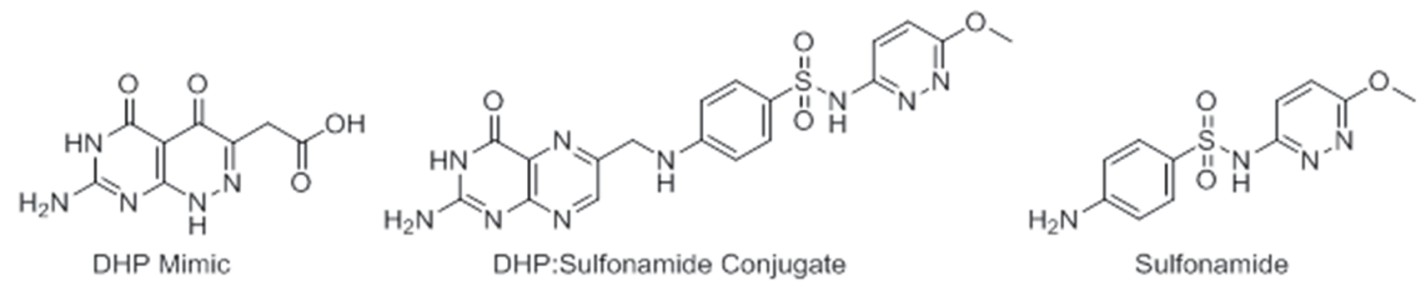

Figure 3-10. Example molecules included in the DHPS inhibitor screen for Pneumocystis activity 
Table 3-4. DHPS inhibitors repurposing screen against Pneumocystis

\begin{tabular}{|c|c|c|c|c|}
\hline Compound & Structure & $\begin{array}{l}\text { Pneumocystis } \\
\mathrm{IC}_{50}(\mu \mathrm{g} / \mathrm{mL})\end{array}$ & $\begin{array}{c}\text { Pneumocystis } \\
\text { \% Inhibition } \\
72 \mathrm{~h} \\
(100 \mu \mathrm{g} / \mathrm{mL})\end{array}$ & $\begin{array}{c}\text { DHPS FP IC } 50 \\
\mu \mathrm{g} / \mathrm{mL} \\
+\mathrm{PPi}\end{array}$ \\
\hline 937 & & 22.8 & 74.91 & 5.42 \\
\hline 1238 & & 2.81 & N/A & 3.02 \\
\hline 1481 & & 6.32 & 94.35 & 8.52 \\
\hline 1522 & & N/A & 78.18 & 6.27 \\
\hline 1525 & & 3.26 & 92.08 & 9.89 \\
\hline 1530 & & $\mathrm{~N} / \mathrm{A}$ & 91.97 & 3.57 \\
\hline 1578 & & N/A & 69.22 & 1.16 \\
\hline 1830 & & 10.33 & N/A & 9.69 \\
\hline 1838 & & 15.59 & N/A & 11.40 \\
\hline
\end{tabular}


structure is interesting, it contains a long aliphatic chains off a tertiary amine, which could favor blood brain barrier penetration and lysosomal trappgng but could also give rise to a high volume of distribution. Since PCP is a pulmonary disease, compounds need to be able to accumulate in lung tissues and have a high free fraction available to be successful. Early PK analysis of this compound and its distribution patterns in various tissues of interest could determine its future as a lead like scaffold. Concurrently, shrinking or eliminating the aliphatic chains from that position could modulate BBB penetration without a significant loss in activity. Structural modifications will begin there as well as modulation of the aromatic nitro group. Compound 14207 has excellent chemical tractability, in that it contains many sites that are easily manipulated to generate extensive SAR. This is excellent to start producing analogs that can raise its TI and improve on drug like properties. Interestingly, 14207 shares a good deal of structural similarity to the MmpL3 inhibitor, tetrahydropyrazolo[1,5- a]pyrimidine-3-carboxamide (THPP) (Figure 3-11). This is intriguing due to the proposed primary target of THPP being mycolic acid transport, something that $P$. carinii does not possess. This could add credence to the theory that THPP is polypharmacologic, similar to SQ109 [162]. Resistant mutants to 14207 in P. carinii could unveil an alternate mechanism of antibacterial activity that could be helpful in PCP and TB drug discovery. Discoveries such as this are a huge positive for this type of screening effort, gaining unique insights into the biology of these pathogens in serendipitous ways.

As for our target based repurposing efforts, success will be further gauged as new compounds are procured. We have identified 9 strong hits from our repurposing efforts that will continue to be validated and investigated. The $\mathrm{IC}_{50 \mathrm{~S}}$ in $P$. carinii closely matched the $\mathrm{IC}_{50}$ s of fluorescent polarization assays performed on purified DHPS (data not shown), which gives confidence that these are on target. The most interesting development from this screening effort was the difference in activities between the sulfonamide drugs and their corresponding pterin conjugates. These conjugates are rife with solubility issues, rendering their enzymatic and whole cell data difficult to interpret at times. However, almost unilaterally they were more potent than their corresponding lone sulfonamide. This could go back to slight differences in the DHPS isoforms between bacteria and Pneumocystis, or it could be an artifact of the in vitro assay conditions tied to the conjugates poor solubility. In vivo testing should flesh out which of these series is a more suitable lead going forward.

Recent efforts in the lab to begin fragment based drug discovery on the DHPS enzyme give rise to an interesting possibility for more selective $P$. carinii/jirovecii agents. As discussed previously, the known DHFR inhibitor TMP is significantly less active in PCP than in other infections due to its lower affinity for the P. jirovecii isoform of the enzyme. There has been work to develop new pcDHFR selective inhibitors, using structure guided design [257]. DHPS is much more highly conserved across bacterial species, but the possibility remains to have a drug emerge with higher pcDHPS affinity that can be used more preferentially in cases of PCP. With the tremendous resources provided in both fragment based drug discovery and structure guided medicinal chemistry optimization, known inhibitors of a critical enzyme such as DHPS should be employed against as many pathogenic species as possible to find their best applications. 

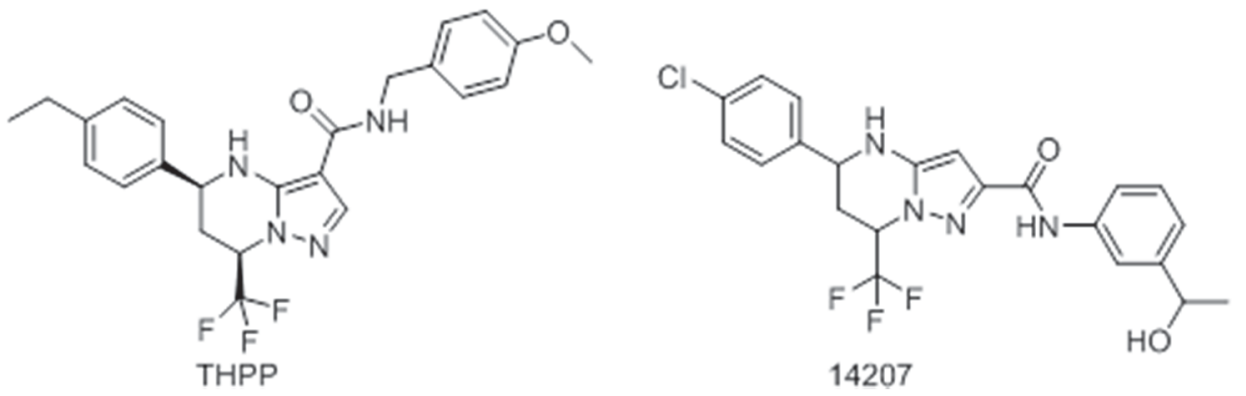

Figure 3-11. Structural comparision of 14207 to THPP

THPP is a preclinical MmpL3 inhibitor with suspected polypharmacology. Pneumocystis has no mycolic acids, so establishing a common means of antimicrobial activity could give insights into both compounds mechanisms of action. 
Many under explored or emerging diseases should be able to harness screens like this, using conserved pathways with known inhibitors.

\section{Discussion on Antibacterial Drug Discovery and Repurposing}

As drug resistance becomes more of issue in the years coming, finding new ways to attack pathogens becomes imperative. One of the most successful approaches to identify new antibiotics is to revisit pathways that are known to be critical for survival and have a strong clinical track record of being targeted. At the same time, you must be cognizant of the very real threat of cross resistance or easily mutable targets. The folate biosynthesis pathway is an excellent example of a highly druggable pathway with significant clinical relevance. Bactrim has been a staple therapy for decades, in a host of different infections both bacterial and otherwise. Retargeting this known pathway is very likely to lead to something with a high degree of clinical relevance, especially with combination therapies in mind.

A novel HPPK inhibitor could provide a similar degree of synergy when combined with TMP if it is able to significantly deplete the levels of DHF that TMP competes with at the DHFR. The added benefit here would be sulfonamide resistant infections should in theory be susceptible to this alternative therapy. Additionally those patients that already suffer from sulfonamide allergies would be able to have a stop-gap therapy option before escalating to stronger agents. Not only would this be a quality of life improvement for the patient, this could help belay resistance to last line of defense agents such as daptomycin and the carbapenems as fewer bacterial populations would be exposed to them. With the power of structure guided design coupled with a good set of fragments to develop, the opportunity for fragment development and optimization is one that is incredibly promising on this essential enzyme. As higher and higher affinity is achieved, potent inhibitors that emerge will concurrently serve as important tools in studying the enzymes function and importance to many pathogenic organisms. We have already described successful repurposing of DHPS inhibitors in $P$. carinii so there is hope that such a broadly essential and conserved enzyme could have demonstrative effects on more than just bacterial infections. Tight binders can also be repurposed for biochemical screening. A high affinity molecule that suffers from poor physiochemical properties that disfavor it as a drug lead can be useful in designing probes for HTS, something our group has done previously $[262,263]$. Such tools can be invaluable in the discovery of new chemical entities that push discoveries and understanding forward.

The difficulty of translating target activity to whole cell potency phenomenon is observed in our design of anti-folate antibiotics. We have established modest inhibitors of the HPPK enzyme, many of which bind tightly to the purified enzyme. However we fail to replicate similar potencies in the whole cell, despite this being an essential enzyme. Newer studies are being designed to test how much inhibition is actually occurring in the cell [264], which could help answer many questions for compound series such as these. We have restarted this project using fragment based drug design, screening the aforementioned 4,500 fragments we have to identify tight binders. With crystallography 
in-house, the next set of HPPK inhibitors will be optimized with efficiency in mind as well as target occupancy and whole cell target inactivation. It is a nice academic pursuit to identify enzyme inhibitors; they can be used for mechanism studies and other non-drug like utilities. If the end goal is a standalone therapeutic agent, than more stringent filters need to be applied to projects from the get go if a series ever hopes to be clinically relevant.

Drug repurposing can be done in two ways: phenotypically looking for whole cell response (death in our case) or more target based using biochemical assays to assess specific enzymatic modulation. Each way contains its own advantages and disadvantages, and each has produced results in terms of interesting molecules. However, it is evident that better approaches need to be incorporated in both, as there is still a tremendous lack of innovation in emerging anti-bacterial drug compounds. Advances in our knowledge of the disease biology are becoming more and more appreciated in setting up these types of screens. Knowing which pathways could be useful in what organisms, or knowing conditions to run your assays to properly recapitulate physiological relevance is integral, and is something the field is becoming better at. The folate biosynthesis pathway has been drugged for almost 80 years, and just recently it was hypothesiszed that the sulfonamide's best antibacterial contribution could be from something other than DHPS inhibition [226]. Such a revelation is startling, but can be capitalized on. Further understanding of synergy mechanisms can be exploited as mentioned earlier, and it opens up the possibility of a new druggable target downstream of DHPS. I believe our drug repurposing screens highlights new ways of screening that can be incredibly powerful. Using whole cell screens of known active compounds (enzymatic with weak bacterial inhibition or bioactive with unknown mechanism) we immediately identify useful compound series and start to observe SAR trends that make an impact on the whole organism, rather than just purified enzyme. With the use of targeted libraries such as our legacy DHPS inhibitors we were able to flesh out a biosynthetic pathway of importance in a different organism than the project originally sought out to inhibit. This rational approach exemplifies the benefits of a target screen that are often overlooked due to lack of success the approach has suffered, while still harnessing the intrinsic advantages of phenotypic screening. The positives from an academic standpoint are; less deconvolution of the mechanism of action and pre-guided SAR to generate hypotheses since you are working with a library of known enzymatic inhibitors. In this case we suspect a slightly different isoform of the enzyme could be occurring in Pneumocystis which we hope to investigate further by purifying the pcDPHS to make sure our SAR rules hold true. 


\section{Materials and Methods}

\section{HPPK}

\section{Enzyme preparation}

The EcHPPK-GST fusion gene was kindly provided by Dr.Honggao Yan. The EcHPPK-GST fusion enzyme was expressed in E.coli and purified as previously described in three steps: (1) GSTrap FF column (Amersham Biosciences), (2) thrombin digestion to remove the GST tag, and (3) size exclusion chromatography (Hevener, et al., 2010; Li, et al., 2003).

\section{Enzyme assay}

HPPK activity was determined by measuring unprocessed ATP substrate using the Kinase-Glo Luminescent assay kit (Promega)(Chhabra, et al., 2012). The enzyme activity of HPPK was measured in a reaction mixture of $50 \mu$ l containing $0.75 \mu \mathrm{M} 6-$ hydroxymethyl-7,8-dihydropterin hydrochloride, $0.75 \mu \mathrm{M}$ ATP, $100 \mathrm{mM}$ Tris, $\mathrm{pH} 8.5,10$ $\mathrm{mM} \mathrm{MgCl}$, $10 \mathrm{mM}$ BME, 0.01\%(V/V) Tween 20, 0.01\%(W/V) BSA, 5\% DMSO, and 5 ng EcHPPK. Inhibitor compounds were dissolved in DMSO, and inhibition was tested at $250 \mu \mathrm{M}$. After 20 minutes of incubation at $23^{\circ} \mathrm{C}$, the unprocessed ATP was detected using the Kinase-Glo assay kit with a plate reader spectrophotometer (Thermo Scientific) according to manufacturer's suggested protocols. To determine the half maximal inhibitory concentration (IC50) values, EcHPPK activities were measured in the presence of various concentrations of the compounds. Data were analyzed using GraphPad Prism software.

\section{Biotinylation of HPPK}

HPPK was minimally biotinylated by reaction with EZ-Link Sul-fo-NHS-LC-LCBiotin (Thermo Scientific). The biotin reagent was added to the protein at a 0.5:1 molar ratio, and the reaction was incubated on ice for $5 \mathrm{~h}$. Unconjugated biotin was removed by pro-cessing the samples through two Zeba Spin Desalting Columns (Thermo Scientific) that had been equilibrated with storage buffer ( $20 \mathrm{mM}$ Tris ( $\mathrm{pH} 8.0), 100 \mathrm{mM} \mathrm{NaCl}, 10 \%$ glycerol). Bovine serum albumin (BSA) was added to the reaction at a final concentration of $0.1 \mathrm{mg} / \mathrm{mL}$ immediately prior to processing through the spin col-umns to improve

recovery. ${ }^{34}$ The biotinylated protein was aliquot-ted, flash-frozen and stored at $80 \mathrm{LC}$ for use in subsequent binding experiments.

\section{Affinity analysis of compound binding by surface plasmon resonance}

SPR experiments were conducted at 20 LC using a SensiQ Pioneer optical biosensor (SensiQ Technologies). Neutravidin (Thermo Sci-entific) was covalently immobilized on a polysaccharide hydrogel-coated gold surface (COOH5 chip; SensiQ Technologies) using routine amine coupling chemistry in immobilization buffer $(10 \mathrm{mM}$ HEPES pH 7.4, $150 \mathrm{mM} \mathrm{NaCl}, 0.005 \%$ Tween20). Carboxyl groups on the hydrogel were activated with $\mathrm{N}$-ethyl-N ${ }^{0}$-(3-dimethylamino-propyl) carbodiimide (EDC) and N- 
hydroxysuccinimide (NHS), and neutravidin was injected until immobilization levels of 5000- 7000 RU were achieved. Remaining active sites were blocked by reaction with ethanolamine. The instrument was primed with binding buffer $(20 \mathrm{mM}$ Tris $\mathrm{pH} 8.5,100$ $\mathrm{mM} \mathrm{NaCl}, 10 \mathrm{mM} \mathrm{MgCl} 2,1 \mathrm{mM}$ TCEP, $0.005 \%$ Tween 20, 10\% glycerol, 5\% DMSO), and biotinylated HPPK was injected until 3000-3700 RU of protein was captured. Unoccupied biotin-binding sites of neutravidin on both the reference and HPPK surfaces were blocked with amine-PEG 2 -biotin (Thermo Scientific) to minimize potential nonspecific binding by the compounds. ${ }^{35}$ For the binding of compounds 5, 6, 7, 8, 10 and 20 to HPPK in the absence of AMPCPP, the data were collected in screening mode (i.e. a single injection at $100 \mu \mathrm{M})$. For all other experiments, the compounds were prepared in running buffer as a 3-fold dilution series starting at $100 \mu \mathrm{M}$ and were injected at a flow rate of $80 \mu \mathrm{L} / \mathrm{min}$. A series of buffer-only (blank) injections was included throughout the experiment to account for instrumental noise. The data were processed, doublereferenced, solvent cor-rected and analyzed ${ }^{35,36}$ using the software package Qdat (version 2.1.0.21, BioLogic Software). The equilibrium dissociation constants were determined by fitting the data to a 1:1 interaction model.

\section{Crystallographic analyses}

The purified protein was concentrated to $6 \mathrm{mg} / \mathrm{ml}$ and crystals were grown by the vapor diffusion method at $18^{\circ} \mathrm{C}$. EcHPPK was incubated with $4 \mathrm{mM}$ AMPCPP, $20 \mathrm{mM}$ $\mathrm{MgCl}$, and 1 2 $\mathrm{mM}$ inhibitor (final 4\% DMSO). Initial crystals were identified robotically using the PEGs suite, PEGs II suite, and JCSG+ suite crystallization screening kits (Qiagen), and these were manually optimized. The co-crystallization conditions for compounds 1, 15, 19, 22 and 23 are $0.1 \mathrm{M}$ Tris, $\mathrm{pH} 8.5,0.2 \mathrm{M} \mathrm{CaCl}$, and 25\% PEG 4K, in a 1:1 ratio with the protein complex solution. The condition for compounds 2 and 34 is $0.1 \mathrm{M}$ Hepes, $\mathrm{pH} 7.5,0.2 \mathrm{M} \mathrm{CaCl}$, and $25 \% \mathrm{PEG} 4 \mathrm{~K}$. The condition for compound 33 is $0.1 \mathrm{M}$ sodium cacodylate, $\mathrm{pH} 6.5$, and $1 \mathrm{M}$ tri-sodium citrate. Crystals were cryoprotected with $30 \%$ glycerol, and then flash frozen in liquid nitrogen. All diffraction data were collected at the SERCAT beam lines 22-ID and 22-BM at the Advanced Photon Source, and processed using HKL2000(Otwinowski and Minor, 1997). Structures were solved by molecular replacement using the EcHPPK structure as the search model. Structures were refined and optimized using PHENIX(Adams, et al., 2002) and COOT(Emsley and Cowtan, 2004), respectively. Data collection and refinement statistics are presented in Table 2.

\section{Virtual fragment screening}

Based on the hit fragment 6-thioguanine that binds at the pterin-binding site, a 2D pharmacophore search was conducted against the NCI database $(265,242$ compounds) for fragment-like molecules (Molecular weight cutoff) yielding 25 compounds. The resulting hits were docked into the pterin site receptor derived from EcHPPK / 6thioguanine structure using AutoDock 3.05 and ranked with the native scoring function. Compounds that were found unable to bind to the site in these calculations were excluded, and the remaining 17 fragment-like compounds were acquired from the Drug Synthesis and Chemistry Branch, Developmental Therapeutics Program, Division of Cancer Treatment and Diagnosis, NCI and tested against the purified EcHPPK. 


\section{Synthesis of compounds: Materials and instrumentation}

All chemicals and solvents were purchased from commercial sources. The chemical reactions were tracked by TLC using Silicycle Silica Gel 60 F254 plates and spots were visualized by UV lamp or I2 condensation. 1H NMR were recorded on a 400 $\mathrm{MHz}$ Bruker NMR and chemical shifts were reported relative to solvent peak. Analytical RP-HPLC was determined on a Waters Acquity UPLC system equipped with an Acquity BEH C18 column $(1.7 \mu \mathrm{m})$, flow rate of $0.5 \mathrm{~mL} / \mathrm{min}$ and a gradient of solvent $\mathrm{A}$ (water with $0.1 \%$ formic acid) and solvent B (acetonitrile with $0.1 \%$ formic acid): $0-0.25 \mathrm{~min}$ 97\% A; 0.25-3.0 min 3-100\% B (linear gradient); 3.0-4.5 min 100\% B; 4.5-4.75 min 097\% A (linear gradient); 4.75-5.0 min 97\% A. Both UV absorbance (monitored at $225-$ $475 \mathrm{~nm}$ ) and ELSD were used as detection methods. All compounds were found to have $>95 \%$ purity with the described analytical methods.

\section{Synthesis of compounds: General procedure}

In a round bottom flask equipped with a stir bar was dissolved 2-amino-8mercapto-1H-purin-6(7H)-one $(2 \mathrm{mmol})$ in $10 \mathrm{~mL}$ of $.4 \mathrm{~N}$ sodium hydroxide in water and 2-bromo-1-substituted ethanone $(2.5 \mathrm{mmol})$ dissolved in $2 \mathrm{~mL}$ of EtOH was added. Mixture stirred for one hour at room temperature. Solution was neutralized with drop wise addition of $1 \mathrm{~N} \mathrm{HCl}$ to yield precipitate which was collected via vacuum filtration and was washed with diethyl-ether and verified to be pure via LC-MS and NMR.

Below is a listing of analytical purity of compounds discussed in this section. Experimental details for determination are described in the methods above.

2-amino-8-((2-oxo-2-(p-tolyl)ethyl)thio)-1H-purin-6(7H)-one:

${ }^{1} \mathrm{H}$ NMR (400 MHz, DMSO-d6) $\delta 2.38$ (s, 3H), 2.55 (s, 4H), 4.67 (s, 2H), 6.05 (s, 2H), $7.32(\mathrm{~d}, \mathrm{~J}=7.94 \mathrm{~Hz}, 2 \mathrm{H}), 7.87-8.00(\mathrm{~m}, 2 \mathrm{H}), 10.57$ (s, 1H). ESI-MS: [M+H]+ Found: 316.3

2-amino-8-((2-(4-hydroxyphenyl)-2-oxoethyl)thio)-1H-purin-6(7H)-one:

${ }^{1} \mathrm{H}$ NMR (400 MHz, DMSO-d6) $\delta 4.71$ (s, 2H), 6.36 (s, 2H), $6.70-6.79$ (m, 2H), 7.79 7.88 (m, 2H). ESI-MS: [M+H]+ Found: 318.2

2-amino-8-((2-(4-fluorophenyl)-2-oxoethyl)thio)-1H-purin-6(7H)-one:

${ }^{1} \mathrm{H}$ NMR (400 MHz, DMSO-d6) $\delta 4.75$ (s, 2H), 6.41 (s, 2H), $7.27-7.34$ (m, 2H), 8.05 (dd, $\mathrm{J}=5.63,8.71 \mathrm{~Hz}, 2 \mathrm{H})$. ESI-MS: $[\mathrm{M}+\mathrm{H}]+$ Found: 320.2

4-(2-((2-amino-6-oxo-6,7-dihydro-1H-purin-8-yl)thio)acetyl)benzonitrile:

1H NMR (400 MHz, DMSO-d6) $\delta 4.89$ (s, 2H), 6.29 (s, 2H), 8.03 - 8.06 (m, 2H), 8.15 $8.19(\mathrm{~m}, 2 \mathrm{H}), 10.60(\mathrm{~d}, \mathrm{~J}=27.50 \mathrm{~Hz}, 2 \mathrm{H}), 12.35$ - 12.64 (m, 1H). ESI-MS: [M+H]+ Found: 327.2 
2-amino-8-((2-(4-methoxyphenyl)-2-oxoethyl)thio)-1H-purin-6(7H)-one:

1H NMR (400 MHz, DMSO-d6) $\delta 3.87$ (s, 3H), 4.82 (d, J = 19.12 Hz, 2H), $6.32(\mathrm{~s}, 1 \mathrm{H})$, $6.96-7.27(\mathrm{~m}, 2 \mathrm{H}), 8.00(\mathrm{dd}, \mathrm{J}=6.36,8.71 \mathrm{~Hz}, 2 \mathrm{H}), 10.48(\mathrm{~s}, 1 \mathrm{H}), 12.53(\mathrm{~s}, 1 \mathrm{H})$. ESIMS: $[\mathrm{M}+\mathrm{H}]+$ Found: 332.2

2-amino-8-((2-(4-(2-morpholinoethoxy)phenyl)-2-oxoethyl)thio)-1H-purin-6(7H)-one: 1H NMR (400 MHz, DMSO-d6) $\delta 2.42(\mathrm{~d}, \mathrm{~J}=5.07 \mathrm{~Hz}, 4 \mathrm{H}), 2.60(\mathrm{dt}, \mathrm{J}=6.42,30.99 \mathrm{~Hz}$, $2 \mathrm{H}), 3.53(\mathrm{t}, \mathrm{J}=4.51 \mathrm{~Hz}, 4 \mathrm{H}), 4.01(\mathrm{t}, \mathrm{J}=6.27 \mathrm{~Hz}, 1 \mathrm{H}), 4.25(\mathrm{t}, \mathrm{J}=6.30 \mathrm{~Hz}, 1 \mathrm{H}), 4.70-$ $4.90(\mathrm{~m}, 2 \mathrm{H}), 6.13(\mathrm{~s}, 1 \mathrm{H}), 6.86(\mathrm{dd}, \mathrm{J}=2.73,8.78 \mathrm{~Hz}, 2 \mathrm{H}), 7.89(\mathrm{dd}, \mathrm{J}=4.31,7.68 \mathrm{~Hz}$, 2H), 10.76 (s, 1H). ESI-MS: [M+H]+ Found: 431.2

2-amino-8-((2-morpholino-2-oxoethyl)thio)-1H-purin-6(7H)-one: $1 \mathrm{H} \mathrm{NMR}(400 \mathrm{MHz}$, DMSO-d6) $\delta 3.45(\mathrm{~d}, \mathrm{~J}=4.97 \mathrm{~Hz}, 3 \mathrm{H}), 3.49-3.59(\mathrm{~m}, 6 \mathrm{H}), 3.61(\mathrm{~d}, \mathrm{~J}=4.71 \mathrm{~Hz}, 3 \mathrm{H})$, $4.22(\mathrm{~s}, 3 \mathrm{H}), 6.32(\mathrm{~s}, 2 \mathrm{H}), 10.52(\mathrm{~s}, 1 \mathrm{H}), 12.52(\mathrm{~s}, 1 \mathrm{H})$. ESI-MS: $[\mathrm{M}+\mathrm{H}]+$ Found: 311.2

2-amino-8-((2-oxo-2-(m-tolyl)ethyl)thio)-1H-purin-6(7H)-one:

1H NMR (400 MHz, DMSO-d6) $\delta 2.39$ (s, 3H), 4.87 (s, 1H), $6.28-6.39$ (m, 1H), $7.41-$ 7.52 (m, 2H), $7.81-7.94$ (m, 2H), 10.57 (s, 1H). ESI-MS: [M+H]+ Found: 316.1

2-amino-8-((2-oxo-2-(o-tolyl)ethyl)thio)-1H-purin-6(7H)-one:

1H NMR (400 MHz, DMSO-d6) $\delta 2.37$ (s, 3H), 4.71 (s, 2H), 6.33 (s, 1H), $7.28-7.38$ (m, 2H), $7.46(\mathrm{td}, \mathrm{J}=1.38,7.57 \mathrm{~Hz}, 1 \mathrm{H}), 7.91(\mathrm{~d}, \mathrm{~J}=7.60 \mathrm{~Hz}, 1 \mathrm{H}), 10.55(\mathrm{~d}, \mathrm{~J}=42.42$ $\mathrm{Hz}, 1 \mathrm{H}), 12.69$ (d, J = $132.88 \mathrm{~Hz}, 1 \mathrm{H})$. ESI-MS: [M+H]+ Found: 316.1

2-amino-8-((2-(2-methoxyphenyl)-2-oxoethyl)thio)-1H-purin-6(7H)-one:

1H NMR (400 MHz, DMSO-d6) $\delta 3.93$ (s, 3H), 4.68 (s, 2H), 6.28 (s, 2H), 6.89 - 7.39 $(\mathrm{m}, 2 \mathrm{H}), 7.47-7.86(\mathrm{~m}, 2 \mathrm{H}), 10.57(\mathrm{~s}, 1 \mathrm{H}), 12.58(\mathrm{~s}, 1 \mathrm{H})$. ESI-MS: $[\mathrm{M}+\mathrm{H}]+$ Found: 332.1

2-amino-8-((1-oxo-1-phenylpropan-2-yl)thio)-1H-purin-6(7H)-one:

1H NMR (400 MHz, DMSO-d6) $\delta 1.54(\mathrm{~d}, \mathrm{~J}=6.84 \mathrm{~Hz}, 3 \mathrm{H}), 5.44(\mathrm{q}, \mathrm{J}=6.89 \mathrm{~Hz}, 1 \mathrm{H})$, $6.35(\mathrm{~s}, 2 \mathrm{H}), 7.52(\mathrm{t}, \mathrm{J}=7.74 \mathrm{~Hz}, 2 \mathrm{H}), 7.60-7.70(\mathrm{~m}, 1 \mathrm{H}), 7.96-8.06(\mathrm{~m}, 2 \mathrm{H}), 10.68(\mathrm{~s}$, $1 \mathrm{H}), 12.66$ (s, 1H). ESI-MS: [M+H]+ Found: 316.0

2-amino-8-((1-oxo-1-(p-tolyl)propan-2-yl)thio)-1H-purin-6(7H)-one:

1H NMR (400 MHz, DMSO-d6) $\delta 1.53(\mathrm{dd}, \mathrm{J}=2.07,7.01 \mathrm{~Hz}, 3 \mathrm{H}), 2.38$ (d, J = $2.05 \mathrm{~Hz}$, $3 \mathrm{H}), 5.43(\mathrm{~s}, 1 \mathrm{H}), 6.34(\mathrm{~s}, 2 \mathrm{H}), 7.27-7.43(\mathrm{~m}, 2 \mathrm{H}), 7.91(\mathrm{dd}, \mathrm{J}=2.04,8.33 \mathrm{~Hz}, 2 \mathrm{H})$, $10.60(\mathrm{~s}, 1 \mathrm{H}), 12.49(\mathrm{~d}, \mathrm{~J}=31.18 \mathrm{~Hz}, 1 \mathrm{H})$. ESI-MS: [M+H]+ Found: 330.0

S-(2-amino-6-oxo-6,7-dihydro-1H-purin-8-yl) 4-fluorobenzothioate:

1H NMR (400 MHz, DMSO-d6) $\delta 6.45-6.61$ (m, 2H), $7.20-7.46$ (m, 2H), $7.89-8.07$ (m, 2H), 10.73 (s, 1H), 12.97 (s, 1H). ESI-MS: [M+H]+ Found: 306.2 
2-amino-8-((2-morpholinoethyl)thio)-1H-purin-6(7H)-one:

1H NMR (400 MHz, DMSO-d6) $\delta 2.35-2.47$ (m, 4H), 2.61 (t, J = 6.88 Hz, 2H), 3.56 (t, $\mathrm{J}=4.62 \mathrm{~Hz}, 4 \mathrm{H}), 6.32(\mathrm{~s}, 2 \mathrm{H}), 10.60(\mathrm{~s}, 1 \mathrm{H}), 12.53$ (s, 1H). ESI-MS: [M+H]+ Found:

297.2

\section{Repurposing Screening}

\section{Bioactive molecule selection}

The selection of compounds was performed with simple structural clustering using Schrödinger Maestro software package. Unique clusters were analyzed for therapeutic index by comparing their $\mathrm{IC}_{50}$ values in drug sensitive (MAR 3D7) and drug resistant (MAR K1) P. falciparum to $\mathrm{IC}_{50} \mathrm{~S}$ in multiple human cell lines (BJ, HepG, Hek293). Compounds were obtained from the St Jude REMP storage system at $10 \mathrm{mM}$ in DMSO and prepared for $P$. carinii screening.

\section{DHPS active set}

Known DHPS inhibitors were selected from legacy screens that possessed low $\mathrm{IC}_{50 \mathrm{~S}}$ in the presence of pyrophosphate. These compounds were obtained at either $10 \mathrm{mM}$ or $10 \mu \mathrm{g} / \mathrm{mL}$ and prepared for $P$. carinii screening.

\section{Compound preparation}

Compounds were obtained in $100 \%$ DMSO solution at $10 \mathrm{mM}$ and stored at $4{ }^{\circ} \mathrm{C}$ prior to testing. Compounds were diluted to $50 \mu \mathrm{M}$ for initial testing in RPMI-1640 containing 20\% horse serum, 1\% MEM vitamin solution, 1\% MEM NEAA, and 2,000 units $/ \mathrm{ml}$ Penicillin-Streptomycin. Negative controls were media alone and $10 \mu \mathrm{g} / \mathrm{ml}$ ampicillin. Positive control was $1 \mu \mathrm{g} / \mathrm{ml}$ pentamidine isethionate.

\section{P. carinii ATP assay}

Cryopreserved and characterized $P$. carinii $(P c)$ isolated from rat lung tissue was distributed into triplicate wells of 48 -well plates with a final volume of $500 \mu 1$ and a final concentration of $5 \times 10^{7}$ nuclei $/ \mathrm{ml}$. Controls and test compounds were added and incubated at $37{ }^{\circ} \mathrm{C}$. At 24,48 , and 72 hours, $10 \%$ of the well volume was removed and the ATP content was measured using Perkin Elmer ATP-liteM luciferin-luciferase assay. The luminescence generated by the ATP content of the samples was measured by a BMG PolarStar optima spectrophotometer. A sample of each group was examined microscopically on the final assay day to rule out the presence of bacteria. Hits were qualified as a $50 \%$ reduction after 72 hours and rescreened for dose response to calculate an $\mathrm{IC}_{50}$.

\section{Calculations}

Background luminescence was subtracted and triplicate well readings of duplicate assays were averaged. For each day's readings, \% reduction in ATP for all groups was 
calculated: experimental - experimental/vehicle control x100. 50\% inhibitory concentration $\left(\mathrm{IC}_{50}\right)$ was calculated in INSTAT linear regression program. 


\section{CHAPTER 4. OVERALL DISCUSSION ON ANTIBIOTIC DRUG DISCOVERY: LESSONS AND EXPEIRENCES}

\section{Difficulties Facing the Field}

As discussed extensively throughout this document, there is a disturbing lack of new antibacterial efficacious chemical matter. Almost every antibiotic brought to market since the 1980's has simply been an expansion on previous drug classes. Bacteria continue to develop more and more extensive resistance mechanism to any antibiotic they are exposed to, eventually we will lose this medicinal chemistry arms race. The need for new targets, and novel ways of approaching old and new targets alike is evident There are a multitude of reasons why this field has struggled to progress in the last three decades, and the combination of these issues has left us in the state we find ourselves in currently. The prospects of returning to a "post-antibiotic" era are real, if perhaps overstated at times. The more realistic immediate outcome would be much more rigorous controlled use of our last line agents such as carbapenems and glycopeptides, which might make some procedures more risky and disfavored. I will highlight some commonly associated problems in antibiotic drug discovery and discuss how my experiences in this field have shaped my views on the subject.

\section{Screening Issues}

The 90's and early 2000's brought on the era of HTS. Massive combinatorial and diversity oriented synthesis programs were launched to generate large libraries of chemically unique scaffolds. Corresponding to the shift in chemistry, there was a turning point in biology in the form of whole genome sequencing. Bacteria could be sequenced quickly, and when coupled with other molecular biology techniques such as DNA microarray and gene knockouts, provided a litany of essential drug targets for chemists to pursue [265]. The targets were purified and biochemical assays were developed to launch a highly lauded period of rational drug design. Hopes were high that chemical entities could be isolated that possessed high degrees of target specificity and optimizations could occur to ensure drug like properties. This approach has largely failed as there were often difficulties translating in vitro potency to in vivo cures. Typically the biggest obstacle is ADME properties of lead compounds and difficulties establishing relevant concentrations at the sites of infection. The problems can even be traced back to simple bacterial penetration. As previously discussed gram-negative bacteria are difficult to kill due to their outer membrane and their possession of efflux mechanisms. However, even tightly bound, highly specific inhibitors can have difficulty penetrating and killing gram-positive species. Phenotypic screening has had more relevant success, but is not without its own short comings. Many times these screens produce indiscriminate electrophiles, or compounds that are converted to covalent modifiers that have immediate toxicity concerns. Also physical chemical properties have historically been poor for drugs emerging from such screens, often due to the library composition from the start. 
Selectivity is difficult to achieve using whole cell unbiased screening as well. Clearly, smarter screening strategies are required if we want to produce new chemical matter for drug resistant pathogens.

Target based screening was implicated as the wave of the future, with massive combinatorial chemistry efforts and diversity oriented synthesis producing immense compound libraries combined with advances in HTS techniques and capabilities. Scientists felt they could brute strength screen enough compounds against a known and validated target that eventually a drug-like lead would emerge that was highly target specific and thus safe and efficacious. However most of these target based screens were done using purified protein to study binding, or enzymatic activity. This has led to many highly specific compounds that have poor drug like properties, and even those that do have a good profile in vitro, fail to recapitulate their effects in vivo or even worse, fail to be efficacious or are toxic once they get tested on a human patient. Part of the failure of many of these screens was their inability to recapitulate the true cellular environment, especially in antibacterial drug discovery. Mechanisms such as cellular penetration or xenobiotic efflux are rarely accounted for and metabolic redundancies in the organism can be overlooked. Recent advances in biophysical screening could be implemented to account for cellular penetration [264]. Thermal denaturing assays, or thermal shift assays (TSA), are a popular HTS technique used to find ligands that stabilize a protein complex. Proteins are heated in the presence of a fluorescent dye and either control or drug, eventually the protein will destabilize due to the heat and the exposed hydrophobic sites will excite the dye creating a signal. In the presence of a drug that binds this denaturing will occur at a higher temperature, and the degree of the "shift" in the denaturing temperature can be converted into a binding affinity. Cellular Thermal Shift (CETSA) applies this theory, but incorporates the ability of the compound of interest to not only penetrate the cell but also its ability to reach and bind to the protein. After dosing a cell, heat shock is applied at escalating temperatures after which the cell is lysed and spun down. Denatured protein will crash out of solution and be spun into the pellet and stable protein will still be in the supernatant. From here, Western blot analysis can confirm how much of your protein remained stable, and similar curves can be calculated to give an effective intracellular binding affinity. With advances in proteomics this technique should be able to actually screen libraries of different compounds and determine where active compounds are binding

Whole-cell, phenotypic screening has been discussed previously in the context on Mtb drug discovery. The basics of this technique in the context of antibiotic discovery are to grow bacteria in vitro and drug them with pure compound or compound mixtures (ie natural product crude extracts or fractions). After a specified amount of time (depending on growth rate of the organism) you will examine the bacterial cultures to look for cell death or inhibited growth, whether this is visually or with biochemical assistance. Hits are identified and typically rescreened for dose response confirmation. Mechanism of action for diverse screens is deduced by the growth of bacteria in the presence of sub lethal concentrations of the hit until resistant mutants are colonized that display significant increases in their MIC. These resistant mutants are genetically sequenced and compare to the wild type to see where what domain changes occur in. As with target 
based screening there are examples of success for drug discovery here, it is easy to state that this system has been more successful at generating large amounts active antibiotic drug compounds [266]. The limited approval of bedaquiline is the most recent example of the successes this system can provide, but it also highlights the potential limitations of this approach. Compounds emerging from phenotypic screening tend to be highly lipophilic molecules that are often toxic and polypharmacologic. High lipophillicity as well as multiple aromatic rings are highly correlated with hERG inhibition, a common predictor of cardiac toxicity in humans [267]. HTS libraries are becoming increasingly biased towards both of these conditions, generally due to the bias of the chemists supplying them. Palladium coupling reactions have become exceedingly popular in the synthesis world and tend to produce very flat and often unsaturated molecules [268]. This lack of diversity is problematic for the issues described previously, but there is also a failure to properly evaluate all chemical space. Techniques like FBDD are useful for increasing chemical sampling, but often lack the specific potency to illicit a response in a phenotypic screen. Thus these screens are less likely to pick out specifically interacting compounds and often have to spend many years in optimization to tailor a hit down to specifics and then build back out for optimized PK/PD parameters. Better library curation could be the solution to aiding in phenotypic screening. Instead of using huge $500+\mathrm{Da}$ compounds, smaller and more diverse sets should be designed. Molecules around 300350 Da with a low cLogP $(\sim 3)$ can sample a large amount of chemical space and have the potency to illicit whole cell responses. With the added benefit of structural clustering, medium sized libraries of small, diverse molecules can be set up for HTS; both phenotypically and target based.

\section{Big Pharma Pulling Out}

Another large reason for the decline in antibiotics is the withdrawal of large pharmaceutical companies from research and development. Antibiotics typically offer a small market share to begin with, especially with the emergence of resistance. Physicians want to use as little antibiotics as possible and would want to use new antibiotics as a last resort. This makes for a tough sell to a board of directors to invest a lot of money in a research program. This fact coupled with a lack of tangible results over several decades caused a mass exodus when an economic recession hit [269]. Now only a handful of large companies are invested in research, and when it is estimated to take so long to recoup the cost of developing an antibiotic that is unsurprising. Government agencies around the world are taking notice and are looking for ways to step in and help incentivize discovery and development of new antimicrobials. Academics, smaller biotechnology companies

and non-profits such as the Global TB Alliance have taken up the mantle in the meantime of early discovery and development. Once a developed compound is produced though, it almost always requires a large firm to step in and handle the cost of conducting large scale clinical trials required for approval.

The discovery of late stage toxicity or a failure in Phase II efficacy trials is especially problematic for pharmaceutical companies, who are on the hook for a tremendous amount of money to push lead compounds through clinical trials. It is 
expected that a drug will cost upwards of a billion dollars to reach market approval, and with toxicity concerns coupled with a lower than average return on investment, antibiotic drug discovery is just a risky endeavor for a profit driven entity. To make investments more sound, multiple actions need to be taken.

First, the barriers to entry must be altered to get more innovation stimulated. The FDA is taking this step in antimicrobial development with initiatives such as the Generating Antibiotic Incentives Now (GAIN) provisions signed into law in 2012. This bipartisan legislation grants an expanded period of patent exclusivity for a developer of a new antibiotic against high risk pathogens that are dubbed as Qualified Infectious Disease Products (QIDP). This period is in addition to existing exclusivity protections that can favor orphan diseases and pediatric specific medications. Agents that are covered by GAIN also receive the benefit of expedited regulatory approval in the form of fast track and priority review status in their clinical trials. At the end of 2015 these incentives have resulted in 58 drugs with QIDP status and will hopefully continue to spurn interest and activity in the field.

Secondly, researchers and clinicians need to work together in optimizing clinical trials to have the best patient populations selected for successful results as well as meaningful discoveries. Drug combinations will only become more important in the fight against drug resistance and understanding the underlying mechanisms behind synergy will be integral. Individual patient populations will undoubtedly react differently to drug cocktails; differences in metabolic rates, existing conditions (i.e. renal/liver function), other medications and even the make-up of their micro flora will play roles in efficacy. The effective design of clinical trials for drug repurposing and combination studies could dramatically lower the investment required for drug companies to make a significant impact.

Finally, continued funding and awareness for academic and non-profit research is vital for establishing a strong foundation for drug development to build on. Academics can be more adventurous and take risks early in the process; this can drive the field in unexpected ways. The NIH has seen recent expansions in funding that should aid in this endeavor, but continued advocacy for antimicrobial resistance is still needed. As more young investigators enter the field, they bring with them fresh ideas, fresh perspectives and unique backgrounds that could aid in scientific dogma in this field. These new outlooks could be exactly what the field needs to jump start innovation into unforeseen areas of combating this issue. Ultimately I believe the field is going in the right directions in terms of eliminating previously head ideas that led to poor results. I think surviving through thin periods of funding and pharmaceutical company withdraws forced the field to re-evaluate itself it has emerged craftier. Screens will be smarter going forward, leads will be investigated differently and more carefully scrutinized early and the chemistry going into optimization will be driven by efficiency and with an end goal in mind. 


\section{Discussion on TB Drug Discovery}

There is an obvious need to continue the push to discover novel chemotherapeutic options to treat MDR-TB infections. Resistance continues to spread across the world, and an ever-expanding global population and global strife will only serve to aid in this expansion. This review has highlighted some of the more promising classes of agents being developed by various research groups, but this global effort must be maintained to ensure adequate weapons are available in this ever-evolving fight. Advances in drug discovery approaches, technological tools, a better understanding of the mycobacterium biology and disease states, and some hard learned lessons from failures in clinical trials have made this paradigm appear more promising as of late, and there is reason to hope for improved outcomes for this serious disease are in the pipeline. In the past decade four primary strategies have been applied to discover the new agents discussed previously in this review: target based screening; phenotypic based screening; semisynthetic natural product chemistry driven approach; and repurposing of antibacterial agents. Each of these approaches has its own advantages and disadvantages.

When the whole Mtb genome sequence became available in 1998 [270], it greatly aided the identification of essential genes and facilitated the recognition of many novel Mtb drug targets. This increased target knowledge was thought to hold great potential and ushered in an era of target-based drug discovery primarily through the use of high throughput screening (HTS). However, the HTS target-based approach has to date only produced a few advanced leads with the current most advanced compounds being the InhA inhibitor class discussed earlier [271]. Interestingly, this mirrors the experience of the broader antibacterial drug discovery community, though lessons in this regard were slower to be accepted in the Mtb field. The primary limitations of this approach relate to target selection, screening library composition, and the concomitant hurdle of generating compounds that can penetrate and accumulate in the Mtb bacilli and remain stable to human metabolism. To address these limitations there needs to be a better understanding of which targets are suitable for this approach, chemical libraries need to be improved upon and new approaches like fragment based drug design should be implemented, enabling the building out of high affinity inhibitors with concomitant drug like and Mtb penetrant properties $[272,273]$.

Once the difficulties of target based approaches to TB drug discovery became apparent, whole-cell MIC driven HTS approaches dominated this discovery space, efforts that were fueled by the discovery of bedaquiline. This is in essence a reversion to an old approach that identified INH and EMB, but on a much larger screening scale, taking advantage of vast pharmaceutical screening libraries that have been put together in the intervening decades. The primary advantages of this approach are: is not limited to inherent target bias, thus allowing all potential chemically tractable targets to be explored simultaneously; common efflux and penetration issues are also addressed along with target-sensitivity validation. After an active compound is identified, resistant mutants can be generated and sequenced to identify genetic variations and thus the presumed mechanism of action. The most common targets identified in Mtb via typical phenotypic whole-cell screening were MmpL3, DprE1, and QcrB [164, 274, 275] all of which have 
produced preclinical leads discussed earlier. Another advantage of this method includes the relatively low costs associated with this approach. Many companies have legacy compound libraries can be quickly repurposed for TB drug discovery, which can be compared to published hit data sets for comparative purposes to identify novel leads. Disadvantages include: an often difficult in vitro to in vivo active transition; mitigating off target pharmacology as the hits are often derived from compound classes generated for other human disease indications; repeated discovery of compounds targeting the same enzymes (MmpL3, DprE1, and QcrB); frequent discovery of prodrug activated electrophiles, for which care must be taken to ensure these agents are not activated by central human metabolism, though it must be noted as evidenced by the nitroimidazoles, delaminid and pretomanid this is achievable.

These challenges are often compounded in lead optimization process, as it is common to increase lipophilicity from the initial HTS hit [276], and drug discovery in TB is no different. The NITD recommends monitoring lipophilic efficiency to guide phenotypic-hit selection and development. Development of such compounds will be a delicate balance of maintaining potency and optimizing exposure via ADME (absorption, distribution, metabolism, and excretion) properties. As shown in Figure 4-1, there has been a trend in developing new oral agents from phenotypic screening to better adhere to Lipinski's rule of five, such as BTZ043, Azaindoles and TCA1 [223, 277]. However it should be noted that most of the newer agents are still on the high end of lipophilicity range $(\mathrm{cLogP}>3)$. This not only makes formulation difficult and increases the risk of drug-drug interactions, but may also play a role in limiting drug distribution into the caesum microenviornment. The success of bedaquiline and delaminid are examples that high lipophillicity (cLogP values of 7.3 and 5.6, respectively) can be overcome with advances in formulation to achieve proper oral-exposure levels and advanced medicinal chemistry to block sites of oxidative metabolism. However, they have had limited success in reducing treatment duration, as in vitro and in vivo mouse efficacy testing may have suggested. The results of Q203 in clinical trials could add more evidence to the theory that these large lipophillic molecules have trouble clearing mycobacterium from necrotic granulomas. The feasibility of combining multiple high-liphophilic drugs in MDR-TB combination therapy will likely need to be addressed. This is due to addative liabilities and consequent drug-drug interactions causing decreased bioavailability and additive toxicities if such combinations of lipohilic drugs are to be attempted. These complications, combined with the more favorable distribution across multiple microenviornments, favors the identification and prioritization of phenotypic MIC screening hits with more hydrophilic properties. All of these are concerns when it comes to the isocamphonyl urea series discussed earlier, and is just more evidence that drug discovery is not simply finding the most potent compounds and making them work. Much more goes into a final drug which is why efficiency metrics like LIPE are so important at early stages of drug discovery. If a target cannot be efficiently inhibited than it may not be worthy of extensive studies and medicinal chemistry efforts.

During the golden age of antibiotic discovery, many unique natural product scaffolds were discarded in favor of compounds with more favorable properties or activity. Recent efforts to optimize some of these previously identified natural products 


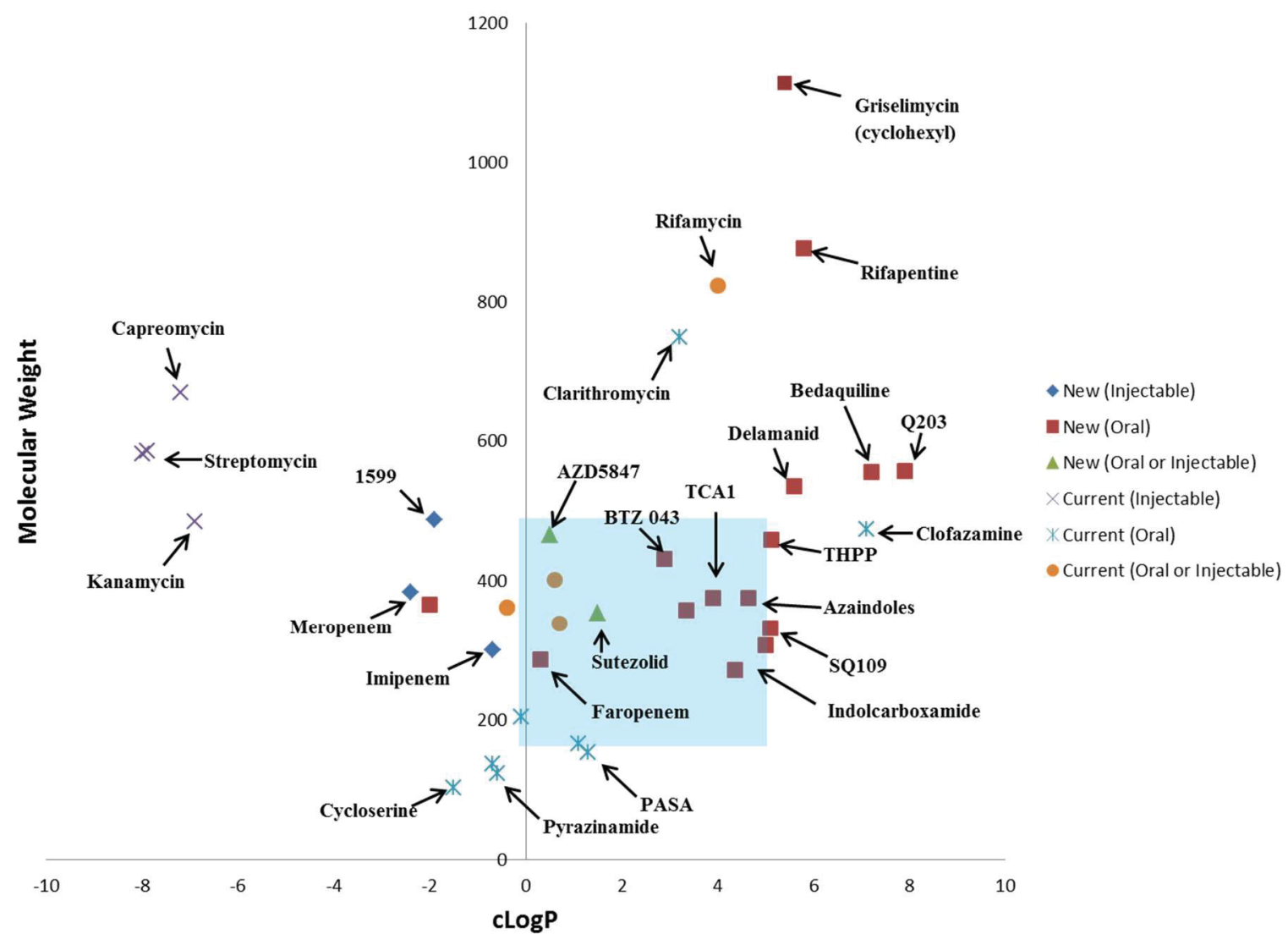

Figure 4-1. Physical chemical properties of currently used TB therapeutics vs newer agents in the discovery pipeline

With their route of administration. The blue-shaded areas indicate the stereotypical Lipinski's rules for oral bioavailability. cLogP values gathered from the pubChem database. 
for MDR-TB therapy, using a semisynthetic chemical modification approach has also proven highly productive, yielding the macrolide SEQ-9, spectinamides, griselimycins, and capuramycins, all of which are suitable to treat MDR-TB. The advantages of these molecules include: in vitro to in vivo efficacy conversion is higher than with purely synthetic compounds; they hit targets complementary to those identified in phenotypic screens; they represent novel chemical classes and with different physicochemical properties from those found in synthetic screening libraries. The primary disadvantages include: these are often resource intensive and difficult programs to initiate; the natural product scaffolds are often of high molecular weight, contain metabolic hotspots and have poor consequent oral bioavailability requiring parenteral administration; this approach often employs difficult and sometimes costly chemistry to generate these analogs. However the above examples of success in structural modifications to turn natural products into preclinical candidates lend great hope to this branch of study. The recent discovery of the so-called "natural" INH in pyridomycins is just more evidence that we can use bacteria to fight other bacterium. Advances in culturing capabilities and more rigorous stress inductions could result in many more natural scaffolds to tweak with medicinal chemistry to get optimal activity.

Repurposing existing antibiotics such as $\beta$-lactams (faropenem, meropenem), oxazolidiones (linezolid), and fluoroquinolones (moxifloxacin) is another productive strategy to identify new MDR-TB treatment options [95, 278]. Linezolid is the prototypical example in terms of MDR-TB treatment [34]. Its proven efficacy in the treatment of MDR-TB has generated further studies on sutezolid and AZD5847, second generation oxazolidiones with greater mycobacterium selectivity and lower side effect potential. This strategy has the advantage of very rapid advancement into clinical trials, as ample human safety and PK data often exists. One factor that limits this approach is the number of new antibacterial agents that are available for repurposing, as the number of new antibacterial agents approved has dropped significantly in recent years. Currently the best untapped opportunity for this approach probably lies in new wave of $\beta$-lactamase inhibitors being developed to tackle multidrug resistant Gram-negative bacterial infections. It remains to be seen if they have sufficient in vivo potency against $\mathrm{Mtb} \mathrm{BlaC}$ enzyme $[279,280]$ to enable future $\beta$-lactam $\beta$-lactamase inhibitor combinations to move forward for MDR-TB. However, the failure of the recent REMoxTB Phase III clinical trial designed to reduce treatment time is a cautionary note for this approach [174].

At the time of this writing, $10 \mathrm{Mtb}$ drug clinical trials are registered in the United States (Table 7), including six compounds classified as "novel chemical entities" by the Stop TB Partnership. There are also 3 Phase III trials for optimized combination studies ongoing with bedaquiline and delamindib and pretomanid (formerly PA-824, another nitroimidazole) being added to optimized background regimens for patients with MDRTB infections. These studies are seeking to enroll more than 1,000 patients suffering from MDR-TB, including some who are co-infected with HIV (NCT00910871, NCT01600963, and NCT01424670). This progress is much improved over past decades, but the need for new drugs that are safer and more efficacious with the ability for treatment shortening is still apparent. A strong discovery pipeline requires many more agents in early stage trials, as attrition rates increase the further drugs progress down the 
pipeline. Thus we believe more new entities are urgently required as some of these agents will inevitably fail.

In conclusion, it is evident that the discovery and development of new agents to treat $\mathrm{Mtb}$ is a very active drug discovery field, with the primary goal of developing a shorter, more effective MDR-TB therapy regimen. Shortening treatment makes patient care easier, reduces the chance of resistance emergence and will decrease the costs associated with addressing this epidemic in the long-term. With the ability to correctly assess where current drugs and their emerging counterparts accumulate and are most effective, clinicians will be able to devise more optimized combination therapies, with particular Mtb life cycles and microenvironments in mind. This field is however not without its challenges, as evidenced by the small numbers of new drugs currently in early stage clinical trials (only 2 compounds in phase I), and by the pharmacological redundancy of many of the new agents in the pipeline that share either similar mechanism of action or side effect profiles, suggesting that significant hurdles still exist and further investment in drug discovery is warranted. Caseum penetration and killing activity has emerged as a key concept in clearing latent bacterial populations and should be examined during the early stages of preclinical evaluations of individual lead compounds and drug classes as a whole. Combination therapy is required for MDR-TB treatment; therefore, how all these new agents may be best clinically combined while minimizing the number of trials needed to be performed remains a key challenge moving forward.

\section{Conclusion and Reflections}

With so much to learn and understand, the challenge to develop agents that will help stymie the flow of antibiotic resistance seems daunting. However, everyday new discoveries reveal an unforeseen path towards progress. With the work being done all over the increasing connected and collaborative world, researchers have an opportunity to discuss and innovate like never before. Nothing will ever be solved by one person or idea, but the free exchange and transmission of ideas will lead to a more complete understanding that will push the boundaries of what we often think we know. I have been granted an unbelievable opportunity to not only learn about drug discovery, but also dive into this unique and challenging world. I have gained experience in a multitude of concentrations, but my focus has always been the chemistry behind making these drugs work. I think the evolution of the field will be continued synergy between chemistry and biology, the more both field knows and understands about the other will be much more significant than increasing knowledge in one concentration alone. I have been incredibly fortunate to be in a highly multi-disciplinary position, and I have learned skills in and an appreciation for all aspects of early stage drug discovery. I have had wonderful guidance and the ability to pursue independent ideas, which I am immensely grateful for.

I have always viewed drug discovery as an intricate and large puzzle; one that you only receive a few pieces at a time and don't have the completed image on the box to guide you. Some pieces are potency of a compound, others are target validation and essentiality and still others are physical properties. The successful completion of this 
puzzle of course requires dedication and patience, but it also mandates the ability to step back and look at the larger picture, often many times. Pieces that seemingly fit together will ultimately be found to be misleading and require rearrangement of your perceptions. Serendipity is ubiquitous in science; it is no exception in this field. Sometimes we must simply be grateful when we find a corner piece that lets many pieces fall in place logically around it. Other times the least likely scenario is the one that ends up solving a large portion of the puzzle. As the puzzle comes together more and more concepts and ideas begin to make sense, and often you can continue to grow and implement these ideas in similar parts of the big picture. It has been a fun and unique challenge for me these past few years, which has shaped me into a better and more inquisitive person and scientist. It is my hope to carry these skills forward into all my future endeavors and continue to foster the multi-disciplinary and collaborative approaches I believe to be crucial in all areas of science. 


\section{LIST OF REFERENCES}

[1] J.E. Lesch, The first miracle drugs : how the sulfa drugs transformed medicine, Oxford University Press, Oxford; New York, 2007.

[2] L.L. Silver, Challenges of Antibacterial Discovery, Clinical Microbiology Reviews, 24 (2011) 71-109.

[3] M. Poe, Antibacterial synergism: a proposal for chemotherapeutic potentiation between trimethoprim and sulfamethoxazole, Science, 194 (1976) 533-535.

[4] G.A. Pankey, L.D. Sabath, Clinical Relevance of Bacteriostatic versus Bactericidal Mechanisms of Action in the Treatment of Gram-Positive Bacterial Infections, Clinical Infectious Diseases, 38 (2004) 864-870.

[5] A.D. Bermingham, J.P., The folic acid biosynthesis pathway in bacteria: evaluation of potential for antibacterial drug discovery, Bioessays, 24 (2002) 637648.

[6] Choquet-Kastylevsky, T. Vial, J. Descotes, Allergic adverse reactions to sulfonamides, Curr Allergy Asthma Rep, 2 (2002) 16-25.

[7] M.-K. Yun, Y. Wu, Z. Li, Y. Zhao, M.B. Waddell, A.M. Ferreira, R.E. Lee, D. Bashford, S.W. White, Catalysis and Sulfa Drug Resistance in Dihydropteroate Synthase, Science, 335 (2012) 1110-1114.

[8] C.J. Thomson, Trimethoprim and brodimoprim resistance of gram-positive and gram-negative bacteria, Journal of chemotherapy (Florence, Italy), 5 (1993) 458464.

[9] T.J. Silhavy, D. Kahne, S. Walker, The Bacterial Cell Envelope, Cold Spring Harbor Perspectives in Biology, 2 (2010) a000414.

[10] T.J. Beveridge, Structures of Gram-Negative Cell Walls and Their Derived Membrane Vesicles, Journal of Bacteriology, 181 (1999) 4725-4733.

[11] K.L. Knutson, Z. Hmama, P. Herrera-Velit, R. Rochford, N.E. Reiner, Lipoarabinomannan of Mycobacterium tuberculosisPromotes Protein Tyrosine Dephosphorylation and Inhibition of Mitogen-activated Protein Kinase in Human Mononuclear Phagocytes: ROLE OF THE Src HOMOLOGY 2 CONTAINING TYROSINE PHOSPHATASE 1, Journal of Biological Chemistry, 273 (1998) 645-652.

[12] A.G. Brown, D. Butterworth, M. Cole, G. Hanscomb, J.D. Hood, C. Reading, NATURALLY-OCCURRING BETA-LACTAMASE INHIBITORS WITH ANTIBACTERIAL ACTIVITY, Journal of Antibiotics, 29 (1976) 668-669.

[13] J.P. Nadler, J. Berger, J.A. Nord, R. Cofsky, M. Saxena, AMoxicillin-clavulanic acid for treating drug-resistant mycobacterium tuberculosis, Chest, 99 (1991) 1025-1026.

[14] M.C. Payen, S. De Wit, C. Martin, R. Sergysels, I. Muylle, Y. Van Laethem, N. Clumeck, Clinical use of the meropenem-clavulanate combination for extensively drug-resistant tuberculosis, The international journal of tuberculosis and lung disease : the official journal of the International Union against Tuberculosis and Lung Disease, 16 (2012) 558-560.

[15] A. Kaushik, N. Makkar, P. Pandey, N. Parrish, U. Singh, G. Lamichhane, Carbapenems and Rifampicin Exhibit Synergy against Mycobacterium 
tuberculosis and Mycobacterium abscessus, Antimicrob Agents Chemother, (2015).

[16] S.M. Drawz, R.A. Bonomo, Three Decades of $\beta$-Lactamase Inhibitors, Clinical Microbiology Reviews, 23 (2010) 160-201.

[17] D. Kahne, C. Leimkuhler, W. Lu, C. Walsh, Glycopeptide and Lipoglycopeptide Antibiotics, Chemical reviews, 105 (2005) 425-448.

[18] M. Arthur, P. Reynolds, P. Courvalin, Glycopeptide resistance in enterococci, Trends in Microbiology, 4 (1996) 401-407.

[19] G.G. Zhanel, S. Trapp, A.S. Gin, M. DeCorby, P.R.S. Lagacé-Wiens, E. Rubinstein, D.J. Hoban, J.A. Karlowsky, Dalbavancin and telavancin: novel lipoglycopeptides for the treatment of Gram-positive infections, Expert Review of Anti-infective Therapy, 6 (2008) 67-81.

[20] J.N. Steenbergen, J. Alder, G.M. Thorne, F.P. Tally, Daptomycin: a lipopeptide antibiotic for the treatment of serious Gram-positive infections, Journal of Antimicrobial Chemotherapy, 55 (2005) 283-288.

[21] U. Bertsche, S.-J. Yang, D. Kuehner, S. Wanner, N.N. Mishra, T. Roth, M. Nega, A. Schneider, C. Mayer, T. Grau, A.S. Bayer, C. Weidenmaier, Increased Cell Wall Teichoic Acid Production and D-alanylation Are Common Phenotypes among Daptomycin-Resistant Methicillin-Resistant Staphylococcus aureus (MRSA) Clinical Isolates, PLoS ONE, 8 (2013) e67398.

[22] P. Nissen, J. Hansen, N. Ban, P.B. Moore, T.A. Steitz, The Structural Basis of Ribosome Activity in Peptide Bond Synthesis, Science, 289 (2000) 920-930.

[23] I. Chopra, M. Roberts, Tetracycline Antibiotics: Mode of Action, Applications, Molecular Biology, and Epidemiology of Bacterial Resistance, Microbiology and Molecular Biology Reviews, 65 (2001) 232-260.

[24] R.E.W. Hancock, Aminoglycoside uptake and mode of action-with special reference to streptomycin and gentamicin: I. Antagonists and mutants, Journal of Antimicrobial Chemotherapy, 8 (1981) 249-276.

[25] T. Pape, W. Wintermeyer, M.V. Rodnina, Conformational switch in the decoding region of $16 \mathrm{~S}$ rRNA during aminoacyl-tRNA selection on the ribosome, Nat Struct Mol Biol, 7 (2000) 104-107.

[26] M.-P. Mingeot-Leclercq, Y. Glupczynski, P.M. Tulkens, Aminoglycosides: Activity and Resistance, Antimicrobial Agents and Chemotherapy, 43 (1999) 727-737.

[27] J. Palomino, A. Martin, Drug Resistance Mechanisms in Mycobacterium tuberculosis, Antibiotics, 3 (2014) 317.

[28] M.A. Kohanski, D.J. Dwyer, J.J. Collins, How antibiotics kill bacteria: from targets to networks, Nature reviews. Microbiology, 8 (2010) 423-435.

[29] Z. Gu, R. Harrod, E.J. Rogers, P.S. Lovett, Anti-peptidyl transferase leader peptides of attenuation-regulated chloramphenicol-resistance genes, Proceedings of the National Academy of Sciences, 91 (1994) 5612-5616.

[30] T. Tenson, M. Lovmar, M. Ehrenberg, The Mechanism of Action of Macrolides, Lincosamides and Streptogramin B Reveals the Nascent Peptide Exit Path in the Ribosome, Journal of Molecular Biology, 330 (2003) 1005-1014.

[31] S. Bailly, J.J. Pocidalo, M. Fay, M.A. Gougerot-Pocidalo, Differential modulation of cytokine production by macrolides: interleukin- 6 production is increased by 
spiramycin and erythromycin, Antimicrobial Agents and Chemotherapy, 35 (1991) 2016-2019.

[32] E.E. McKee, M. Ferguson, A.T. Bentley, T.A. Marks, Inhibition of Mammalian Mitochondrial Protein Synthesis by Oxazolidinones, Antimicrobial Agents and Chemotherapy, 50 (2006) 2042-2049.

[33] D.R. Ashtekar, R. Costa-Periera, T. Shrinivasan, R. Iyyer, N. Vishvanathan, W. Rittel, Oxazolidinones, a new class of synthetic antituberculosis agent. In vitro and in vivo activities of DuP-721 against Mycobacterium tuberculosis, Diagnostic microbiology and infectious disease, 14 (1991) 465-471.

[34] M. Lee, J. Lee, M.W. Carroll, H. Choi, S. Min, T. Song, L.E. Via, L.C. Goldfeder, E. Kang, B. Jin, H. Park, H. Kwak, H. Kim, H.S. Jeon, I. Jeong, J.S. Joh, R.Y. Chen, K.N. Olivier, P.A. Shaw, D. Follmann, S.D. Song, J.K. Lee, D. Lee, C.T. Kim, V. Dartois, S.K. Park, S.N. Cho, C.E. Barry, 3rd, Linezolid for treatment of chronic extensively drug-resistant tuberculosis, N Engl J Med, 367 (2012) 15081518.

[35] P.C. Rose, U.M. Hallbauer, J.A. Seddon, A.C. Hesseling, H.S. Schaaf, Linezolidcontaining regimens for the treatment of drug-resistant tuberculosis in South African children, The international journal of tuberculosis and lung disease : the official journal of the International Union against Tuberculosis and Lung Disease, 16 (2012) 1588-1593.

[36] H.A. Anger, F. Dworkin, S. Sharma, S.S. Munsiff, D.M. Nilsen, S.D. Ahuja, Linezolid use for treatment of multidrug-resistant and extensively drug-resistant tuberculosis, New York City, 2000-06, The Journal of antimicrobial chemotherapy, 65 (2010) 775-783.

[37] A.J. Garcia-Prats, P.C. Rose, A.C. Hesseling, H.S. Schaaf, Linezolid for the treatment of drug-resistant tuberculosis in children: a review and recommendations, Tuberculosis (Edinburgh, Scotland), 94 (2014) 93-104.

[38] T.A. Mukhtar, G.D. Wright, Streptogramins, Oxazolidinones, and Other Inhibitors of Bacterial Protein Synthesis, Chemical reviews, 105 (2005) 529-542.

[39] R. Wenzel, G. Bate, P. Kirkpatrick, Tigecycline, Nature reviews. Drug discovery, 4 (2005) 809-810.

[40] R.E. Lee, J.G. Hurdle, J. Liu, D.F. Bruhn, T. Matt, M.S. Scherman, P.K. Vaddady, Z. Zheng, J. Qi, R. Akbergenov, S. Das, D.B. Madhura, C. Rathi, A. Trivedi, C. Villellas, R.B. Lee, Rakesh, S.L. Waidyarachchi, D. Sun, M.R. McNeil, J.A. Ainsa, H.I. Boshoff, M. Gonzalez-Juarrero, B. Meibohm, E.C. Bottger, A.J. Lenaerts, Spectinamides: a new class of semisynthetic antituberculosis agents that overcome native drug efflux, Nat Med, 20 (2014) 152-158.

[41] K. Drlica, X. Zhao, DNA gyrase, topoisomerase IV, and the 4-quinolones, Microbiology and Molecular Biology Reviews, 61 (1997) 377-392.

[42] H.G. Floss, T.-W. Yu, RifamycinMode of Action, Resistance, and Biosynthesis, Chemical reviews, 105 (2005) 621-632.

[43] M. Niemi, Pharmacokinetic Interactions with Rifampicin: Clinical Relevance, Clinical Pharmacokinetics, 42 (2003) 819-851.

[44] R.A.M. Breen, Tuberculosis and HIV Co-Infection: A Practical Therapeutic Approach, Drugs, 66 (2006) 2299-2308. 
[45] D.M. Burger, Pharmacokinetic interaction between rifampin and zidovudine, Antimicrobial Agents and Chemotherapy, 37 (1993) 1426-1432.

[46] K. Gallicano, Induction of zidovudine glucuronidation and amination pathways by rifampicin in HIV-infected patients, British Journal of Clinical Pharmacology, 48 (1999) 168-178.

[47] M.A. Kohanski, D.J. Dwyer, B. Hayete, C.A. Lawrence, J.J. Collins, A Common Mechanism of Cellular Death Induced by Bactericidal Antibiotics, Cell, $130797-$ 810.

[48] M.A. Kohanski, D.J. Dwyer, J. Wierzbowski, G. Cottarel, J.J. Collins, Mistranslation of membrane proteins and two-component system activation trigger aminoglycoside-mediated oxidative stress and cell death, Cell, 135 (2008) 679-690.

[49] K.C. Lamp, C.D. Freeman, N.E. Klutman, M.K. Lacy, Pharmacokinetics and Pharmacodynamics of the Nitroimidazole Antimicrobials, Clinical Pharmacokinetics, 36 (2012) 353-373.

[50] A. Koul, N. Dendouga, K. Vergauwen, B. Molenberghs, L. Vranckx, R. Willebrords, Z. Ristic, H. Lill, I. Dorange, J. Guillemont, D. Bald, K. Andries, Diarylquinolines target subunit c of mycobacterial ATP synthase, Nat Chem Biol, 3 (2007) 323-324.

[51] A.H. Diacon, A. Pym, M. Grobusch, R. Patientia, R. Rustomjee, L. Page-Shipp, C. Pistorius, R. Krause, M. Bogoshi, G. Churchyard, A. Venter, J. Allen, J.C. Palomino, T. De Marez, R.P. van Heeswijk, N. Lounis, P. Meyvisch, J. Verbeeck, W. Parys, K. de Beule, K. Andries, D.F. Mc Neeley, The diarylquinoline TMC207 for multidrug-resistant tuberculosis, N Engl J Med, 360 (2009) 23972405.

[52] K. Andries, P. Verhasselt, J. Guillemont, H.W.H. Göhlmann, J.-M. Neefs, H. Winkler, J. Van Gestel, P. Timmerman, M. Zhu, E. Lee, P. Williams, D. de Chaffoy, E. Huitric, S. Hoffner, E. Cambau, C. Truffot-Pernot, N. Lounis, V. Jarlier, A Diarylquinoline Drug Active on the ATP Synthase of Mycobacterium tuberculosis, Science, 307 (2005) 223-227.

[53] S. Watanabe, M. Zimmermann, M.B. Goodwin, U. Sauer, C.E. Barry, 3rd, H.I. Boshoff, Fumarate Reductase Activity Maintains an Energized Membrane in Anaerobic Mycobacterium tuberculosis, PLoS pathogens, 7 (2011) e1002287.

[54] N. Lounis, T. Gevers, J. Van Den Berg, K. Andries, Impact of the Interaction of R207910 with Rifampin on the Treatment of Tuberculosis Studied in the Mouse Model, Antimicrobial Agents and Chemotherapy, 52 (2008) 3568-3572.

[55] J. Cohen, Approval of Novel TB Drug Celebrated-With Restraint, Science, 339 (2013) 130.

[56] L. Preiss, J.D. Langer, Ö. Yildiz, L. Eckhardt-Strelau, J.E.G. Guillemont, A. Koul, T. Meier, Structure of the mycobacterial ATP synthase Fo rotor ring in complex with the anti-TB drug bedaquiline, 2015.

[57] C.L. Ventola, The Antibiotic Resistance Crisis: Part 1: Causes and Threats, Pharmacy and Therapeutics, 40 (2015) 277-283.

[58] WHO, Antimicrobial Resistance, WHO Fact Sheet, (2015).

[59] T.P. Van Boeckel, S. Gandra, A. Ashok, Q. Caudron, B.T. Grenfell, S.A. Levin, R. Laxminarayan, Global antibiotic consumption 2000 to 2010: an analysis of 
national pharmaceutical sales data, The Lancet Infectious Diseases, 14 (2014) 742-750.

[60] C.-E. Luyt, N. Bréchot, J.-L. Trouillet, J. Chastre, Antibiotic stewardship in the intensive care unit, Critical Care, 18 (2014) 480.

[61] F. Luciani, S.A. Sisson, H. Jiang, A.R. Francis, M.M. Tanaka, The epidemiological fitness cost of drug resistance in Mycobacterium tuberculosis, Proceedings of the National Academy of Sciences, 106 (2009) 14711-14715.

[62] W.H. Organization, Antimicrobial Resistance: Global Report on Surveillance 2014, in: http://www.who.int/drugresistance/documents/surveillancereport/en/, 2014.

[63] L. Zhu, J. Lin, J. Ma, J.E. Cronan, H. Wang, Triclosan Resistance of Pseudomonas aeruginosa PAO1 Is Due to FabV, a Triclosan-Resistant EnoylAcyl Carrier Protein Reductase, Antimicrobial Agents and Chemotherapy, 54 (2010) 689-698.

[64] S. Kojima, H. Nikaido, Permeation rates of penicillins indicate that Escherichia coli porins function principally as nonspecific channels, Proceedings of the National Academy of Sciences, 110 (2013) E2629-E2634.

[65] M. Baroud, I. Dandache, G.F. Araj, R. Wakim, S. Kanj, Z. Kanafani, M. Khairallah, A. Sabra, M. Shehab, G. Dbaibo, G.M. Matar, Underlying mechanisms of carbapenem resistance in extended-spectrum $\beta$-lactamaseproducing Klebsiella pneumoniae and Escherichia coli isolates at a tertiary care centre in Lebanon: role of OXA-48 and NDM-1 carbapenemases, International Journal of Antimicrobial Agents, 41 (2013) 75-79.

[66] M. Bubonja-Sonje, M. Matovina, I. Skrobonja, B. Bedenic, M. Abram, Mechanisms of Carbapenem Resistance in Multidrug-Resistant Clinical Isolates of Pseudomonas aeruginosa from a Croatian Hospital, Microbial Drug Resistance, 21 (2015) 261-269.

[67] T. Ferenci, K. Phan, How Porin Heterogeneity and Trade-Offs Affect the Antibiotic Susceptibility of Gram-Negative Bacteria, Genes, 6 (2015) 1113.

[68] M.J. Miller, F. Malouin, Microbial iron chelators as drug delivery agents: the rational design and synthesis of siderophore-drug conjugates, Accounts of Chemical Research, 26 (1993) 241-249.

[69] M. Mallea, J. Chevalier, C. Bornet, A. Eyraud, A. Davin-Regli, C. Bollet, J.-M. Pages, Porin alteration and active efflux: two in vivo drug resistance strategies used by Enterobacter aerogenes, Microbiology, 144 (1998) 3003-3009.

[70] R.A. Weinstein, D.C. Hooper, Efflux Pumps and Nosocomial Antibiotic Resistance: A Primer for Hospital Epidemiologists, Clinical Infectious Diseases, 40 (2005) 1811-1817.

[71] A.V. Vargiu, H. Nikaido, Multidrug binding properties of the AcrB efflux pump characterized by molecular dynamics simulations, Proceedings of the National Academy of Sciences, 109 (2012) 20637-20642.

[72] M.J. Everett, Y.F. Jin, V. Ricci, L.J. Piddock, Contributions of individual mechanisms to fluoroquinolone resistance in 36 Escherichia coli strains isolated from humans and animals, Antimicrobial Agents and Chemotherapy, 40 (1996) 2380-2386. 
[73] L. Pumbwe, L.J.V. Piddock, Two Efflux Systems Expressed Simultaneously in Multidrug-Resistant Pseudomonas aeruginosa, Antimicrobial Agents and Chemotherapy, 44 (2000) 2861-2864.

[74] M. Stavri, L.J.V. Piddock, S. Gibbons, Bacterial efflux pump inhibitors from natural sources, Journal of Antimicrobial Chemotherapy, 59 (2007) 1247-1260.

[75] G.P. Tegos, M. Haynes, J.J. Strouse, M.M. Khan, C.G. Bologa, T.I. Oprea, L.A. Sklar, Microbial efflux pump inhibition: tactics and strategies, Current pharmaceutical design, 17 (2011) 1291-1302.

[76] .M.A. Blair, M.A. Webber, A.J. Baylay, D.O. Ogbolu, L.J.V. Piddock, Molecular mechanisms of antibiotic resistance, Nat Rev Micro, 13 (2015) 42-51.

[77] G.P. Morlock, B. Metchock, D. Sikes, J.T. Crawford, R.C. Cooksey, ethA, inhA, and katG Loci of Ethionamide-Resistant Clinical Mycobacterium tuberculosis Isolates, Antimicrobial Agents and Chemotherapy, 47 (2003) 3799-3805.

[78] D.S. Billal, J. Feng, P. Leprohon, D. Légaré, M. Ouellette, Whole genome analysis of linezolid resistance in Streptococcus pneumoniae reveals resistance and compensatory mutations, BMC Genomics, 12 (2011) 1-10.

[79] W. Gao, K. Chua, J.K. Davies, H.J. Newton, T. Seemann, P.F. Harrison, N.E. Holmes, H.-W. Rhee, J.-I. Hong, E.L. Hartland, T.P. Stinear, B.P. Howden, Two Novel Point Mutations in Clinical Staphylococcus aureus Reduce Linezolid Susceptibility and Switch on the Stringent Response to Promote Persistent Infection, PLoS pathogens, 6 (2010) e1000944.

[80] R. Leclercq, Mechanisms of Resistance to Macrolides and Lincosamides: Nature of the Resistance Elements and Their Clinical Implications, Clinical Infectious Diseases, 34 (2002) 482-492.

[81] T.R. Fritsche, M. Castanheira, G.H. Miller, R.N. Jones, E.S. Armstrong, Detection of Methyltransferases Conferring High-Level Resistance to Aminoglycosides in Enterobacteriaceae from Europe, North America, and Latin America, Antimicrobial Agents and Chemotherapy, 52 (2008) 1843-1845.

[82] M.W. Vetting, S.S. Hegde, M. Wang, G.A. Jacoby, D.C. Hooper, J.S. Blanchard, Structure of QnrB1, a Plasmid-mediated Fluoroquinolone Resistance Factor, Journal of Biological Chemistry, 286 (2011) 25265-25273.

[83] E.P.A.E. Chain, An Enzyme from Bacteria able to Destroy Penicillin, Nature, (1940).

[84] K. Bush, G.A. Jacoby, Updated Functional Classification of $\beta$-Lactamases, Antimicrobial Agents and Chemotherapy, 54 (2010) 969-976.

[85] P. Nordmann, L. Poirel, T.R. Walsh, D.M. Livermore, The emerging NDM carbapenemases, Trends in Microbiology, 19 (2011) 588-595.

[86] G.D. Wright, Bacterial resistance to antibiotics: Enzymatic degradation and modification, Advanced Drug Delivery Reviews, 57 (2005) 1451-1470.

[87] J. Romanowska, N. Reuter, J. Trylska, Comparing aminoglycoside binding sites in bacterial ribosomal RNA and aminoglycoside modifying enzymes, Proteins: Structure, Function, and Bioinformatics, 81 (2013) 63-80.

[88] A.R. Hauser, J. Mecsas, D.T. Moir, Beyond Antibiotics: New Therapeutic Approaches For Bacterial Infections, Clinical Infectious Diseases, (2016).

[89] M.S. Butler, M.A. Blaskovich, M.A. Cooper, Antibiotics in the clinical pipeline in 2013, J Antibiot, 66 (2013) 571-591. 
[90] D.E. Ehmann, H. Jahić, P.L. Ross, R.-F. Gu, J. Hu, T.F. Durand-Réville, S. Lahiri, J. Thresher, S. Livchak, N. Gao, T. Palmer, G.K. Walkup, S.L. Fisher, Kinetics of Avibactam Inhibition against Class A, C, and D $\beta$-Lactamases, The Journal of Biological Chemistry, 288 (2013) 27960-27971.

[91] L.L. Ling, T. Schneider, A.J. Peoples, A.L. Spoering, I. Engels, B.P. Conlon, A. Mueller, T.F. Schaberle, D.E. Hughes, S. Epstein, M. Jones, L. Lazarides, V.A. Steadman, D.R. Cohen, C.R. Felix, K.A. Fetterman, W.P. Millett, A.G. Nitti, A.M. Zullo, C. Chen, K. Lewis, A new antibiotic kills pathogens without detectable resistance, Nature, 517 (2015) 455-459.

[92] I. Comas, M. Coscolla, T. Luo, S. Borrell, K.E. Holt, M. Kato-Maeda, J. Parkhill, B. Malla, S. Berg, G. Thwaites, D. Yeboah-Manu, G. Bothamley, J. Mei, L. Wei, S. Bentley, S.R. Harris, S. Niemann, R. Diel, A. Aseffa, Q. Gao, D. Young, S. Gagneux, Out-of-Africa migration and Neolithic coexpansion of Mycobacterium tuberculosis with modern humans, Nat Genet, 45 (2013) 1176-1182.

[93] WHO, WHO Global Tuberculosis Report 2014, in, 2014.

[94] Z.F. Udwadia, R.A. Amale, K.K. Ajbani, C. Rodrigues, Totally Drug-Resistant Tuberculosis in India, Clinical Infectious Diseases, 54 (2012) 579-581.

[95] A. Zumla, P. Nahid, S.T. Cole, Advances in the development of new tuberculosis drugs and treatment regimens, Nature reviews. Drug discovery, 12 (2013) 388404.

[96] F. Drobniewski, Is Death Inevitable with Multiresistant TB plus HIV Infection, Lancet, 349 (1997) 71-73.

[97] S. Esposito, L. D’Ambrosio, M. Tadolini, H.S. Schaaf, J. Caminero Luna, B. Marais, R. Centis, M. Dara, A. Matteelli, F. Blasi, G.B. Migliori, ERS/WHO Tuberculosis Consilium assistance with extensively drug-resistant tuberculosis management in a child: case study of compassionate delamanid use, European Respiratory Journal, 44 (2014) 811-815.

[98] K. Marisa, W. Robin Mark, H. Cindy, P. Nicolaas Claudius Gey van, S. Elizabeth Maria, M. Borna, S. Frederick Adriaan, C.-N. Mamisa, H. Ebrahim, C. Gerrit, H. Paul David van, V. Thomas Calldo, T. André Phillip, Emergence and Spread of Extensively and Totally Drug-Resistant Tuberculosis, South Africa, Emerging Infectious Disease journal, 19 (2013) 449.

[99] M. Raviglione, XDR-TB: entering the post-antibiotic era?, The international journal of tuberculosis and lung disease : the official journal of the International Union against Tuberculosis and Lung Disease, 10 (2006) 1185-1187.

[100] K. Kumar, I. Abubakar, Clinical implications of the global multidrug-resistant tuberculosis epidemic, Clinical Medicine, 15 (2015) s37-s42.

[101] M.T. Gler, V. Skripconoka, E. Sanchez-Garavito, H. Xiao, J.L. Cabrera-Rivero, D.E. Vargas-Vasquez, M. Gao, M. Awad, S.K. Park, T.S. Shim, G.Y. Suh, M. Danilovits, H. Ogata, A. Kurve, J. Chang, K. Suzuki, T. Tupasi, W.J. Koh, B. Seaworth, L.J. Geiter, C.D. Wells, Delamanid for multidrug-resistant pulmonary tuberculosis, N Engl J Med, 366 (2012) 2151-2160.

[102] N. Ryan, J. Lo, Delamanid: First Global Approval, Drugs, 74 (2014) 1041-1045.

[103] V. Dartois, The path of anti-tuberculosis drugs: from blood to lesions to mycobacterial cells, Nat Rev Micro, 12 (2014) 159-167. 
[104] B.J. Marais, R.P. Gie, H.S. Schaaf, N. Beyers, P.R. Donald, J.R. Starke, Childhood pulmonary tuberculosis: old wisdom and new challenges, American journal of respiratory and critical care medicine, 173 (2006) 1078-1090.

[105] D. Hoagland, Y. Zhao, R. Lee, Advances in Drug Discovery and Development for Pediatric Tuberculosis, Mini Rev Med Chem, (2015).

[106] J.R. Starke, Improving Tuberculosis Care for Children in High-Burden Settings, Pediatrics, 134 (2014) 655-657.

[107] J.A. Seddon, D. Shingadia, Epidemiology and disease burden of tuberculosis in children: a global perspective, Infection and Drug Resistance, 7 (2014) 153-165.

[108] A.M. Mandalakas, J.R. Starke, Current concepts of childhood tuberculosis, Seminars in pediatric infectious diseases, 16 (2005) 93-104.

[109] H.S. Schaaf, R.P. Gie, M. Kennedy, N. Beyers, P.B. Hesseling, P.R. Donald, Evaluation of young children in contact with adult multidrug-resistant pulmonary tuberculosis: a 30-month follow-up, Pediatrics, 109 (2002) 765-771.

[110] O.M. Loto, I. Awowole, Tuberculosis in Pregnancy: A Review, Journal of Pregnancy, 2012 (2012).

[111] H.S. Schaaf, D.P. Parkin, H.I. Seifart, C.J. Werely, P.B. Hesseling, P.D. van Helden, J.S. Maritz, P.R. Donald, Isoniazid pharmacokinetics in children treated for respiratory tuberculosis, Archives of Disease in Childhood, 90 (2005) 614618.

[112] M.R. Holdiness, Clinical pharmacokinetics of the antituberculosis drugs, Clin Pharmacokinet, 9 (1984) 511-544.

[113] D. Corrigan, J. Paton, Hepatic enzyme abnormalities in children on triple therapy for tuberculosis, Pediatric pulmonology, 27 (1999) 37-42.

[114] S. Chien, T.G. Wells, J.L. Blumer, G.L. Kearns, J.S. Bradley, J.A. Bocchini, Jr., J. Natarajan, S. Maldonado, G.J. Noel, Levofloxacin pharmacokinetics in children, Journal of clinical pharmacology, 45 (2005) 153-160.

[115] E.V. Capparelli, M.D. Reed, J.S. Bradley, G.L. Kearns, R.F. Jacobs, B.D. Damle, J.L. Blumer, D.M. Grasela, Pharmacokinetics of Gatifloxacin in Infants and Children, Antimicrobial Agents and Chemotherapy, 49 (2005) 1106-1112.

[116] J.A. Seddon, J.J. Furin, M. Gale, H. Del Castillo Barrientos, R.M. Hurtado, F. Amanullah, N. Ford, J.R. Starke, H.S. Schaaf, Caring for children with drugresistant tuberculosis: practice-based recommendations, American journal of respiratory and critical care medicine, 186 (2012) 953-964.

[117] A.R. Frydenberg, S.M. Graham, Toxicity of first-line drugs for treatment of tuberculosis in children: review Toxicité des médicaments de première ligne pour le traitement de la tuberculose chez les enfants: Revue Toxicidad de los medicamentos de primera línea de tratamiento para tuberculosis en niños: Revisión, Tropical Medicine \& International Health, 14 (2009) 1329-1337.

[118] W.H. Organization, Rapid advice : treatment of tuberculosis in children, in, WHO, Geneva, 2010.

[119] D. Jaganath, E. Mupere, Childhood Tuberculosis and Malnutrition, Journal of Infectious Diseases, 206 (2012) 1809-1815.

[120] R. Basu Roy, Current understanding of the immune response to tuberculosis in children, Current Opinion in Infectious Diseases, 25 (2012) 250-257. 
[121] N.P. Kumar, R. Anuradha, R. Suresh, R. Ganesh, J. Shankar, V. Kumaraswami, T.B. Nutman, S. Babu, Suppressed Type 1, Type 2, and Type 17 Cytokine Responses in Active Tuberculosis in Children, Clinical and Vaccine Immunology, 18 (2011) 1856-1864.

[122] H. Hiruy, Z. Rogers, C. Mbowane, J. Adamson, L. Ngotho, F. Karim, T. Gumbo, W. Bishai, P. Jeena, Subtherapeutic concentrations of first-line anti-TB drugs in South African children treated according to current guidelines: the PHATISA study, Journal of Antimicrobial Chemotherapy, (2014).

[123] W.W. Yew, C.C. Leung, Antituberculosis drugs and hepatotoxicity, Respirology (Carlton, Vic.), 11 (2006) 699-707.

[124] A. Tostmann, M.J. Boeree, R.E. Aarnoutse, W.C. de Lange, A.J. van der Ven, R. Dekhuijzen, Antituberculosis drug-induced hepatotoxicity: concise up-to-date review, Journal of gastroenterology and hepatology, 23 (2008) 192-202.

[125] J.E. Oeltmann, B. Chengeta, J.J. Mboya, C.D. Wells, P.H. Kilmarx, T. Samandari, L.J. Nelson, Reported childhood tuberculosis treatment outcomes, Gaborone and Francistown, Botswana, 1998-2002, The international journal of tuberculosis and lung disease : the official journal of the International Union against Tuberculosis and Lung Disease, 12 (2008) 186-192.

[126] J. Biehl, R.W. Vilter, EFfects of isoniazid on pyridoxine metabolism, Journal of the American Medical Association, 156 (1954) 1549-1552.

[127] J.M. Pellock, J. Howell, J.E.L. Kendig, H. Baker, PYridoxine deficiency in children treated with isoniazid, Chest, 87 (1985) 658-661.

[128] L.G. Possuelo, J.A. Castelan, T.C. de Brito, A.W. Ribeiro, P.I. Cafrune, P.D. Picon, A.R. Santos, R.L. Teixeira, T.S. Gregianini, M.H. Hutz, M.L. Rossetti, A. Zaha, Association of slow N-acetyltransferase 2 profile and anti-TB drug-induced hepatotoxicity in patients from Southern Brazil, European journal of clinical pharmacology, 64 (2008) 673-681.

[129] M.N. Lobato, J.A. Jereb, J.R. Starke, Unintended consequences: mandatory tuberculin skin testing and severe isoniazid hepatotoxicity, Pediatrics, 121 (2008) e1732-1733.

[130] V.J. Palusci, D. O'Hare, R.M. Lawrence, Hepatotoxicity and transaminase measurement during isoniazid chemoprophylaxis in children, The Pediatric infectious disease journal, 14 (1995) 144-148.

[131] P.T.C. Group, Targeted Tuberculin Skin Testing and Treatment of Latent Tuberculosis Infection in Children and Adolescents, Pediatrics, 114 (2004) 11751201.

[132] D. Desrochers, R.P. González-Peralta, D.T. McClenathan, M.J. Wilsey, A. Haafiz, Isoniazid-Induced Severe Hepatotoxicity: An Infrequent but Preventable Cause of Liver Failure in Children Treated for Latent Tuberculosis Infection, Clinical Medicine Insights. Pediatrics, 5 (2011) 9-13.

[133] S.S. Wu, C.S. Chao, J.H. Vargas, H.L. Sharp, M.G. Martin, S.V. McDiarmid, F.R. Sinatra, M.E. Ament, Isoniazid-related hepatic failure in children: a survey of liver transplantation centers, Transplantation, 84 (2007) 173-179.

[134] E. Schuetz, P-glycoprotein: A major determinant of rifampicin-inducible expression of cytochrome P4503A in mice and humans, Proceedings of the National Academy of Sciences USA, 93 (1996) 4001-4006. 
[135] B. Williamson, Induction of Influx and Efflux Transporters and Cytochrome p4503A4 in Primary Human Hepatocytes by Rifampicin, Rifabutin, and Rifapentine., Antimicrobial Agents and Chemotherapy, (2013).

[136] M. Weiner, Effects of Rifampin and Multidrug Resistance Gene Polymorphism on Concentrations of Moxifloxacin, Antimicrobial Agents and Chemotherapy, 51 (2007) 2861-2867.

[137] D. Kuypers, Drug Interaction between mycophenolate mofetil and rifampin: Possible induction of uridine diphosphate-glucuronosyltransferase, Clinical Pharmacology and Therapeutics, 78 (2005) 81-88.

[138] W. Shi, X. Zhang, X. Jiang, H. Ruan, C.E. Barry, H. Wang, W. Zhang, Y. Zhang, Pyrazinamide inhibits trans-translation in Mycobacterium tuberculosis: a potential mechanism for shortening the duration of tuberculosis chemotherapy, Science (New York, N.Y.), 333 (2011) 1630-1632.

[139] Y. Zhang, D. Mitchison, The curious characteristics of pyrazinamide: a review, The International Journal of Tuberculosis and Lung Disease, 7 (2003) 6-21.

[140] I. Sanchez-Albisua, M.L. Vidal, G. Joya-Verde, F. del Castillo, M.I. de Jose, J. Garcia-Hortelano, Tolerance of pyrazinamide in short course chemotherapy for pulmonary tuberculosis in children, The Pediatric infectious disease journal, 16 (1997) 760-763.

[141] S.M. Graham, D.J. Bell, S. Nyirongo, R. Hartkoorn, S.A. Ward, E.M. Molyneux, Low levels of pyrazinamide and ethambutol in children with tuberculosis and impact of age, nutritional status, and human immunodeficiency virus infection, Antimicrob Agents Chemother, 50 (2006) 407-413.

[142] A. Trebucq, Should ethambutol be recommended for routine treatment of tuberculosis in children? A review of the literature, The international journal of tuberculosis and lung disease : the official journal of the International Union against Tuberculosis and Lung Disease, 1 (1997) 12-15.

[143] WHO, Ethambutol efficacy and toxicity : literature review and recommendations for daily and intermittent dosage in children, (2006).

[144] P.R. Donald, H.S. Schaaf, Old and new drugs for the treatment of tuberculosis in children, Paediatric respiratory reviews, 8 (2007) 134-141.

[145] M. Levy, F. Rigaudiere, A. de Lauzanne, B. Koehl, I. Melki, M. Lorrot, A. Faye, Ethambutol-related Impaired Visual Function in Childrens Less than 5 Years of Age Treated for a Mycobacterial Infection: Diagnosis and Evolution, The Pediatric infectious disease journal, 34 (2015) 346-350.

[146] D. Ettehad, H.S. Schaaf, J.A. Seddon, G.S. Cooke, N. Ford, Treatment outcomes for children with multidrug-resistant tuberculosis: a systematic review and metaanalysis, The Lancet Infectious Diseases, 12 449-456.

[147] J.H. Grosset, T.G. Singer, W.R. Bishai, New drugs for the treatment of tuberculosis: hope and reality [State of the Art Series. New tools. Number 2 in the series], The International Journal of Tuberculosis and Lung Disease, 16 (2012) 1005-1014.

[148] M. Raviglione, B. Marais, K. Floyd, K. Lönnroth, H. Getahun, G.B. Migliori, A.D. Harries, P. Nunn, C. Lienhardt, S. Graham, J. Chakaya, K. Weyer, S. Cole, S.H.E. Kaufmann, A. Zumla, Scaling up interventions to achieve global 
tuberculosis control: progress and new developments, The Lancet, 379 19021913.

[149] E.B. Wong, K.A. Cohen, W.R. Bishai, Rising to the challenge: new therapies for tuberculosis, Trends in Microbiology, 21 (2013) 493-501.

[150] A.I. Zumla, S.H. Gillespie, M. Hoelscher, P.P.J. Philips, S.T. Cole, I. Abubakar, T.D. McHugh, M. Schito, M. Maeurer, A.J. Nunn, New antituberculosis drugs, regimens, and adjunct therapies: needs, advances, and future prospects, The Lancet Infectious Diseases, 14 (2014) 327-340.

[151] R. Tasneen, Sterilizing Activity of Novel TMC207- and PA-824-Containing Regimens in a Murine Model of Tuberculosis, Antimicrobial Agents and Chemotherapy, 55 (2011) 5485-5493.

[152] V. Balasubramanian, Effect of Coadministration of Moxifloxacin and Rifampin on the Mycobacterium tuberculosis in a Murine Aersol Infection Model, Antimicrobial Agents and Chemotherapy, 56 (2012) 3054-3058.

[153] H.S. Schaaf, A.J. Garcia-Prats, A.C. Hesseling, J.A. Seddon, Managing multidrug-resistant tuberculosis in children: review of recent developments, Curr Opin Infect Dis, 27 (2014) 211-219.

[154] J.M. Tréluyer, Y. Merlé, S. Tonnelier, E. Rey, G. Pons, Nonparametric Population Pharmacokinetic Analysis of Amikacin in Neonates, Infants, and Children, Antimicrobial Agents and Chemotherapy, 46 (2002) 1381-1387.

[155] C.A. Peloquin, S.E. Berning, A.T. Nitta, P.M. Simone, M. Goble, G.A. Huitt, M.D. Iseman, J.L. Cook, D. Curran-Everett, Aminoglycoside toxicity: daily versus thrice-weekly dosing for treatment of mycobacterial diseases, Clinical infectious diseases : an official publication of the Infectious Diseases Society of America, 38 (2004) 1538-1544.

[156] J.A. Seddon, A.C. Hesseling, B.J. Marais, H. McIlleron, C.A. Peloquin, P.R. Donald, H.S. Schaaf, Paediatric use of second-line anti-tuberculosis agents: a review, Tuberculosis (Edinburgh, Scotland), 92 (2012) 9-17.

[157] K.M. Kam, C.W. Yip, T.L. Cheung, H.S. Tang, O.C. Leung, M.Y. Chan, Stepwise decrease in moxifloxacin susceptibility amongst clinical isolates of multidrug-resistant Mycobacterium tuberculosis: correlation with ofloxacin susceptibility, Microbial drug resistance (Larchmont, N.Y.), 12 (2006) 7-11.

[158] K.A. Cohen, W.R. Bishai, A.S. Pym, Molecular Basis of Drug Resistance in Mycobacterium tuberculosis, Microbiology Spectrum, 2 (2014).

[159] J.D. Fonseca, G.M. Knight, T.D. McHugh, The complex evolution of antibiotic resistance in Mycobacterium tuberculosis, International Journal of Infectious Diseases, 32 (2015) 94-100.

[160] S. Gagneux, C.D. Long, P.M. Small, T. Van, G.K. Schoolnik, B.J. Bohannan, The competitive cost of antibiotic resistance in Mycobacterium tuberculosis, Science, 312 (2006) 1944-1946.

[161] N. Casali, V. Nikolayevskyy, Y. Balabanova, S.R. Harris, O. Ignatyeva, I. Kontsevaya, J. Corander, J. Bryant, J. Parkhill, S. Nejentsev, R.D. Horstmann, T. Brown, F. Drobniewski, Evolution and transmission of drug-resistant tuberculosis in a Russian population, Nat Genet, 46 (2014) 279-286.

[162] K. Li, L.A. Schurig-Briccio, X. Feng, A. Upadhyay, V. Pujari, B. Lechartier, F.L. Fontes, H. Yang, G. Rao, W. Zhu, A. Gulati, J.H. No, G. Cintra, S. Bogue, Y.-L. 
Liu, K. Molohon, P. Orlean, D.A. Mitchell, L. Freitas-Junior, F. Ren, H. Sun, T.

Jiang, Y. Li, R.-T. Guo, S.T. Cole, R.B. Gennis, D.C. Crick, E. Oldfield,

Multitarget Drug Discovery for Tuberculosis and Other Infectious Diseases, Journal of Medicinal Chemistry, 57 (2014) 3126-3139.

[163] G. Riccardi, M.R. Pasca, Trends in discovery of new drugs for tuberculosis therapy, J Antibiot, 67 (2014) 655-659.

[164] K. Mdluli, T. Kaneko, A. Upton, The Tuberculosis Drug Discovery and Development Pipeline and Emerging Drug Targets, Cold Spring Harbor Perspectives in Medicine, (2015).

[165] T.R. Ioerger, T. O’Malley, R. Liao, K.M. Guinn, M.J. Hickey, N. Mohaideen, K.C. Murphy, H.I.M. Boshoff, V. Mizrahi, E.J. Rubin, C.M. Sassetti, C.E. Barry, III, D.R. Sherman, T. Parish, J.C. Sacchettini, Identification of New Drug Targets and Resistance Mechanisms in Mycobacterium tuberculosis, PLoS ONE, 8 (2013) e75245.

[166] Olga T. Schubert, J. Mouritsen, C. Ludwig, Hannes L. Röst, G. Rosenberger, Patrick K. Arthur, M. Claassen, David S. Campbell, Z. Sun, T. Farrah, M. Gengenbacher, A. Maiolica, Stefan H.E. Kaufmann, Robert L. Moritz, R. Aebersold, The Mtb Proteome Library: A Resource of Assays to Quantify the Complete Proteome of Mycobacterium tuberculosis, Cell Host \& Microbe, 13 (2013) 602-612.

[167] K.A. Cohen, T. Abeel, A.M. McGuire, C.A. Desjardins, V. Munsamy, T.P. Shea, B.J. Walker, N. Bantubani, D.V. Almeida, L. Alvarado, S. Chapman, N.R. Mvelase, E.Y. Duffy, M.G. Fitzgerald, P. Govender, S. Gujja, S. Hamilton, C. Howarth, J.D. Larimer, K. Maharaj, M.D. Pearson, M.E. Priest, Q. Zeng, N. Padayatchi, J. Grosset, S.K. Young, J. Wortman, K.P. Mlisana, M.R. O’Donnell, B.W. Birren, W.R. Bishai, A.S. Pym, A.M. Earl, Evolution of extensively drugresistant tuberculosis over four decades revealed by whole genome sequencing of Mycobacterium tuberculosis from KwaZulu-Natal, South Africa, International Journal of Mycobacteriology, 4, Supplement 1 (2015) 24-25.

[168] C.S. Kuan, C.L. Chan, S.M. Yew, Y.F. Toh, J.-S. Khoo, J. Chong, K.W. Lee, Y.C. Tan, W.-Y. Yee, Y.F. Ngeow, K.P. Ng, Genome Analysis of the First Extensively Drug-Resistant (XDR) Mycobacterium tuberculosis in Malaysia Provides Insights into the Genetic Basis of Its Biology and Drug Resistance, PLoS ONE, 10 (2015) e0131694.

[169] D. Schnappinger, K. O'Brien, S. Ehrt, Construction of Conditional Knockdown Mutants in Mycobacteria, in: T. Parish, D.M. Roberts (Eds.) Mycobacteria Protocols, Springer New York, 2015, pp. 151-175.

[170] S. Woong Park, M. Klotzsche, D.J. Wilson, H.I. Boshoff, H. Eoh, U. Manjunatha, A. Blumenthal, K. Rhee, C.E. Barry, III, C.C. Aldrich, S. Ehrt, D. Schnappinger, Evaluating the Sensitivity of Mycobacterium tuberculosis to Biotin Deprivation Using Regulated Gene Expression, PLoS pathogens, 7 (2011) e1002264.

[171] B. Gold, T. Warrier, C. Nathan, A Multi-stress Model for High Throughput Screening Against Non-replicating Mycobacterium tuberculosis, in: T. Parish, D.M. Roberts (Eds.) Mycobacteria Protocols, Springer New York, 2015, pp. 293315. 
[172] B.C. VanderVen, R.J. Fahey, W. Lee, Y. Liu, R.B. Abramovitch, C. Memmott, A.M. Crowe, L.D. Eltis, E. Perola, D.D. Deininger, T. Wang, C.P. Locher, D.G. Russell, Novel Inhibitors of Cholesterol Degradation in Mycobacterium tuberculosis Reveal How the Bacterium's Metabolism Is Constrained by the Intracellular Environment, PLoS pathogens, 11 (2015) e1004679.

[173] B. Prideaux, L.E. Via, M.D. Zimmerman, S. Eum, J. Sarathy, P. O'Brien, C. Chen, F. Kaya, D.M. Weiner, P.-Y. Chen, T. Song, M. Lee, T.S. Shim, J.S. Cho, W. Kim, S.N. Cho, K.N. Olivier, C.E. Barry Iii, V. Dartois, The association between sterilizing activity and drug distribution into tuberculosis lesions, Nat Med, advance online publication (2015).

[174] S.H. Gillespie, A.M. Crook, T.D. McHugh, C.M. Mendel, S.K. Meredith, S.R. Murray, F. Pappas, P.P.J. Phillips, A.J. Nunn, Four-Month Moxifloxacin-Based Regimens for Drug-Sensitive Tuberculosis, New England Journal of Medicine, 371 (2014) 1577-1587.

[175] M.C. Kjellsson, L.E. Via, A. Goh, D. Weiner, K.M. Low, S. Kern, G. Pillai, C.E. Barry, V. Dartois, Pharmacokinetic Evaluation of the Penetration of Antituberculosis Agents in Rabbit Pulmonary Lesions, Antimicrobial Agents and Chemotherapy, 56 (2012) 446-457.

[176] J.-M. Michot, C. Seral, F. Van Bambeke, M.-P. Mingeot-Leclercq, P.M. Tulkens, Influence of Efflux Transporters on the Accumulation and Efflux of Four Quinolones (Ciprofloxacin, Levofloxacin, Garenoxacin, and Moxifloxacin) in J774 Macrophages, Antimicrobial Agents and Chemotherapy, 49 (2005) 24292437.

[177] W.R. Barclay, R.H. Ebert, G.V. Le Roy, R.W. Manthei, L.J. Roth, Distribution and excretion of radioactive isoniazid in tuberculous patients, J Am Med Assoc, 151 (1953) 1384-1388.

[178] G. Canetti, R. Parrot, G. Porven, M. Le Lirzin, [Rifomycin levels in the lung and tuberculous lesions in man], Acta tuberculosea et pneumologica Belgica, 60 (1969) 315-322.

[179] G.A. Ellard, M.J. Humphries, B.W. Allen, Cerebrospinal fluid drug concentrations and the treatment of tuberculous meningitis, The American review of respiratory disease, 148 (1993) 650-655.

[180] G.A. Ellard, M.J. Humphries, M. Gabriel, R. Teoh, Penetration of pyrazinamide into the cerebrospinal fluid in tuberculous meningitis, British medical journal (Clinical research ed.), 294 (1987) 284-285.

[181] B. Prideaux, V. Dartois, D. Staab, D.M. Weiner, A. Goh, L.E. Via, C.E. Barry, 3rd, M. Stoeckli, High-sensitivity MALDI-MRM-MS imaging of moxifloxacin distribution in tuberculosis-infected rabbit lungs and granulomatous lesions, Anal Chem, 83 (2011) 2112-2118.

[182] B. Prideaux, M. Stoeckli, Mass spectrometry imaging for drug distribution studies, Journal of proteomics, 75 (2012) 4999-5013.

[183] H.T. Wright, K.A. Reynolds, Antibacterial Targets in Fatty Acid Biosynthesis, Current opinion in microbiology, 10 (2007) 447-453.

[184] Y.-M. Zhang, S.W. White, C.O. Rock, Inhibiting Bacterial Fatty Acid Synthesis, Journal of Biological Chemistry, 281 (2006) 17541-17544. 
[185] H.-J. Li, C.-T. Lai, P. Pan, W. Yu, N. Liu, G.R. Bommineni, M. Garcia-Diaz, C. Simmerling, P.J. Tonge, A Structural and Energetic Model for the Slow-Onset Inhibition of the Mycobacterium tuberculosis Enoyl-ACP Reductase InhA, ACS Chemical Biology, 9 (2014) 986-993.

[186] R. Rawat, A. Whitty, P.J. Tonge, The isoniazid-NAD adduct is a slow, tightbinding inhibitor of InhA, the Mycobacterium tuberculosis enoyl reductase: Adduct affinity and drug resistance, Proceedings of the National Academy of Sciences, 100 (2003) 13881-13886.

[187] C.-T. Lai, H.-J. Li, W. Yu, S. Shah, G.R. Bommineni, V. Perrone, M. GarciaDiaz, P.J. Tonge, C. Simmerling, Rational Modulation of the Induced-Fit Conformational Change for Slow-Onset Inhibition in Mycobacterium tuberculosis InhA, Biochemistry, 54 (2015) 4683-4691.

[188] H. Lu, P.J. Tonge, Drug-target residence time: critical information for lead optimization, Current Opinion in Chemical Biology, 14 (2010) 467-474.

[189] P.J. Tummino, R.A. Copeland, Residence Time of Receptor-Ligand Complexes and Its Effect on Biological Function, Biochemistry, 47 (2008) 5481-5492.

[190] R.A. Copeland, D.L. Pompliano, T.D. Meek, Drug-target residence time and its implications for lead optimization, Nature reviews. Drug discovery, 5 (2006) 730739.

[191] H. Lu, K. England, C. am Ende, J.J. Truglio, S. Luckner, B.G. Reddy, N.L. Marlenee, S.E. Knudson, D.L. Knudson, R.A. Bowen, C. Kisker, R.A. Slayden, P.J. Tonge, Slow-Onset Inhibition of the FabI Enoyl Reductase from Francisella tularensis: Residence Time and in Vivo Activity, ACS Chemical Biology, 4 (2009) 221-231.

[192] S. Cantaloube, R. Veyron-Churlet, N. Haddache, M. Daffé, D. Zerbib, The Mycobacterium Tuberculosis FAS-II Dehydratases and Methyltransferases Define the Specificity of the Mycolic Acid Elongation Complexes, PLoS ONE, 6 (2011) e29564.

[193] L. Gannoun-Zaki, L. Alibaud, L. Kremer, Point Mutations within the Fatty Acid Synthase Type II Dehydratase Components HadA or HadC Contribute to Isoxyl Resistance in Mycobacterium tuberculosis, Antimicrobial Agents and Chemotherapy, 57 (2013) 629-632.

[194] E. Sacco, A.S. Covarrubias, H.M. O'Hare, P. Carroll, N. Eynard, T.A. Jones, T. Parish, M. Daffé, K. Bäckbro, A. Quémard, The missing piece of the type II fatty acid synthase system from Mycobacterium tuberculosis, Proceedings of the National Academy of Sciences, 104 (2007) 14628-14633.

[195] N. Slama, S. Jamet, W. Frigui, A. Pawlik, D. Bottai, F. Laval, P. Constant, A. Lemassu, K. Cam, M. Daffé, R. Brosch, N. Eynard, A. Quémard, The changes in mycolic acid structures caused by hadC mutation have a dramatic effect on the virulence of Mycobacterium tuberculosis, Molecular Microbiology, 99 (2016) 794-807.

[196] A.L. Dias, D. Jain, Ibrutinib: A New Frontier in the Treatment of Chronic Lymphocytic Leukemia by Bruton's Tyrosine Kinase Inhibition, Cardiovascular \& Hematological Agents in Medicinal Chemistry, 11 (2013) 265-271. 
[197] R.A. Bauer, Covalent inhibitors in drug discovery: from accidental discoveries to avoided liabilities and designed therapies, Drug Discovery Today, 20 (2015) 1061-1073.

[198] L.A. Honigberg, A.M. Smith, M. Sirisawad, E. Verner, D. Loury, B. Chang, S. Li, Z. Pan, D.H. Thamm, R.A. Miller, J.J. Buggy, The Bruton tyrosine kinase inhibitor PCI-32765 blocks B-cell activation and is efficacious in models of autoimmune disease and B-cell malignancy, Proceedings of the National Academy of Sciences, 107 (2010) 13075-13080.

[199] J.A. Di Paolo, T. Huang, M. Balazs, J. Barbosa, K.H. Barck, B.J. Bravo, R.A.D. Carano, J. Darrow, D.R. Davies, L.E. DeForge, L. Diehl, R. Ferrando, S.L. Gallion, A.M. Giannetti, P. Gribling, V. Hurez, S.G. Hymowitz, R. Jones, J.E. Kropf, W.P. Lee, P.M. Maciejewski, S.A. Mitchell, H. Rong, B.L. Staker, J.A. Whitney, S. Yeh, W.B. Young, C. Yu, J. Zhang, K. Reif, K.S. Currie, Specific Btk inhibition suppresses B cell- and myeloid cell-mediated arthritis, Nat Chem Biol, 7 (2011) 41-50.

[200] B.R. Lanning, L.R. Whitby, M.M. Dix, J. Douhan, A.M. Gilbert, E.C. Hett, T.O. Johnson, C. Joslyn, J.C. Kath, S. Niessen, L.R. Roberts, M.E. Schnute, C. Wang, J.J. Hulce, B. Wei, L.O. Whiteley, M.M. Hayward, B.F. Cravatt, A road map to evaluate the proteome-wide selectivity of covalent kinase inhibitors, Nat Chem Biol, 10 (2014) 760-767.

[201] J.M. Bradshaw, J.M. McFarland, V.O. Paavilainen, A. Bisconte, D. Tam, V.T. Phan, S. Romanov, D. Finkle, J. Shu, V. Patel, T. Ton, X. Li, D.G. Loughhead, P.A. Nunn, D.E. Karr, M.E. Gerritsen, J.O. Funk, T.D. Owens, E. Verner, K.A. Brameld, R.J. Hill, D.M. Goldstein, J. Taunton, Prolonged and tunable residence time using reversible covalent kinase inhibitors, Nat Chem Biol, 11 (2015) 525531.

[202] D.E. Scott, A.G. Coyne, S.A. Hudson, C. Abell, Fragment-Based Approaches in Drug Discovery and Chemical Biology, Biochemistry, 51 (2012) 4990-5003.

[203] M. Congreve, R. Carr, C. Murray, H. Jhoti, A 'Rule of Three' for fragment-based lead discovery?, Drug Discovery Today, 8 (2003) 876-877.

[204] L.K. Sharma, R. Leonardi, W. Lin, V.A. Boyd, A. Goktug, A.A. Shelat, T. Chen, S. Jackowski, C.O. Rock, A High-Throughput Screen Reveals New SmallMolecule Activators and Inhibitors of Pantothenate Kinases, Journal of Medicinal Chemistry, 58 (2015) 1563-1568.

[205] S.M. Devine, M.D. Mulcair, C.O. Debono, E.W.W. Leung, J.W.M. Nissink, S.S. Lim, I.R. Chandrashekaran, M. Vazirani, B. Mohanty, J.S. Simpson, J.B. Baell, P.J. Scammells, R.S. Norton, M.J. Scanlon, Promiscuous 2-Aminothiazoles (PrATs): A Frequent Hitting Scaffold, Journal of Medicinal Chemistry, 58 (2015) 1205-1214.

[206] R.C. Hartkoorn, C. Sala, J. Neres, F. Pojer, S. Magnet, R. Mukherjee, S. Uplekar, S. Boy-Röttger, K.-H. Altmann, S.T. Cole, Towards a new tuberculosis drug: pyridomycin - nature's isoniazid, EMBO molecular medicine, 4 (2012) 10321042.

[207] L.A. Woods, O. Dolezal, B. Ren, J.H. Ryan, T.S. Peat, S.-A. Poulsen, Native State Mass Spectrometry, Surface Plasmon Resonance, and X-ray 
Crystallography Correlate Strongly as a Fragment Screening Combination, Journal of Medicinal Chemistry, 59 (2016) 2192-2204.

[208] X. Chen, S. Qin, S. Chen, J. Li, L. Li, Z. Wang, Q. Wang, J. Lin, C. Yang, W. Shui, A Ligand-observed Mass Spectrometry Approach Integrated into the Fragment Based Lead Discovery Pipeline, Scientific Reports, 5 (2015) 8361.

[209] P. Domenech, M.B. Reed, C.E. Barry, Contribution of the Mycobacterium tuberculosis MmpL Protein Family to Virulence and Drug Resistance, Infection and Immunity, 73 (2005) 3492-3501.

[210] R.C. Goldman, Why are membrane targets discovered by phenotypic screens and genome sequencing in Mycobacterium tuberculosis?, Tuberculosis (Edinburgh, Scotland), 93 (2013) 569-588.

[211] R.E. Lee, M. Protopopova, E. Crooks, R.A. Slayden, M. Terrot, C.E. Barry, Combinatorial Lead Optimization of [1,2]-Diamines Based on Ethambutol as Potential Antituberculosis Preclinical Candidates, Journal of Combinatorial Chemistry, 5 (2003) 172-187.

[212] M. Biava, G.C. Porretta, F. Manetti, New derivatives of BM212: A class of antimycobacterial compounds based on the pyrrole ring as a scaffold, Mini Rev Med Chem, 7 (2007) 65-78.

[213] W. Li, A. Upadhyay, F.L. Fontes, E.J. North, Y. Wang, D.C. Crans, A.E. Grzegorzewicz, V. Jones, S.G. Franzblau, R.E. Lee, D.C. Crick, M. Jackson, Novel Insights into the Mechanism of Inhibition of MmpL3, a Target of Multiple Pharmacophores in Mycobacterium tuberculosis, Antimicrobial Agents and Chemotherapy, 58 (2014) 6413-6423.

[214] G. Poce, R.H. Bates, S. Alfonso, M. Cocozza, G.C. Porretta, L. Ballell, J. Rullas, F. Ortega, A. De Logu, E. Agus, V. La Rosa, M.R. Pasca, E. De Rossi, B. Wae, S.G. Franzblau, F. Manetti, M. Botta, M. Biava, Improved BM212 MmpL3 inhibitor analogue shows efficacy in acute murine model of tuberculosis infection, PLoS One, 8 (2013) e56980.

[215] S.P. Rao, S.B. Lakshminarayana, R.R. Kondreddi, M. Herve, L.R. Camacho, P. Bifani, S.K. Kalapala, J. Jiricek, N.L. Ma, B.H. Tan, S.H. Ng, M. Nanjundappa, S. Ravindran, P.G. Seah, P. Thayalan, S.H. Lim, B.H. Lee, A. Goh, W.S. Barnes, Z. Chen, K. Gagaring, A.K. Chatterjee, K. Pethe, K. Kuhen, J. Walker, G. Feng, S. Babu, L. Zhang, F. Blasco, D. Beer, M. Weaver, V. Dartois, R. Glynne, T. Dick, P.W. Smith, T.T. Diagana, U.H. Manjunatha, Indolcarboxamide is a preclinical candidate for treating multidrug-resistant tuberculosis, Science translational medicine, 5 (2013) 214ra168.

[216] S. Lun, H. Guo, O.K. Onajole, M. Pieroni, H. Gunosewoyo, G. Chen, S.K. Tipparaju, N.C. Ammerman, A.P. Kozikowski, W.R. Bishai, Indoleamides are active against drug-resistant Mycobacterium tuberculosis, Nat Commun, 4 (2013).

[217] J.R. Brown, E.J. North, J.G. Hurdle, C. Morisseau, J.S. Scarborough, D. Sun, J. Korduláková, M.S. Scherman, V. Jones, A. Grzegorzewicz, R.M. Crew, M. Jackson, M.R. McNeil, R.E. Lee, The structure-activity relationship of urea derivatives as anti-tuberculosis agents, Bioorganic \& Medicinal Chemistry, 19 (2011) 5585-5595.

[218] M.S. Scherman, E.J. North, V. Jones, T.N. Hess, A.E. Grzegorzewicz, T. Kasagami, I.-H. Kim, O. Merzlikin, A.J. Lenaerts, R.E. Lee, M. Jackson, C. 
Morisseau, M.R. McNeil, Screening a library of 1600 adamantyl ureas for antiMycobacterium tuberculosis activity in vitro and for better physical chemical properties for bioavailability, Bioorganic \& Medicinal Chemistry, 20 (2012) 3255-3262.

[219] A.E. Grzegorzewicz, H. Pham, V.A.K.B. Gundi, M.S. Scherman, E.J. North, T. Hess, V. Jones, V. Gruppo, S.E.M. Born, J. Korduláková, S.S. Chavadi, C. Morisseau, A.J. Lenaerts, R.E. Lee, M.R. McNeil, M. Jackson, Inhibition of mycolic acid transport across the Mycobacterium tuberculosis plasma membrane, Nat Chem Biol, 8 (2012) 334-341.

[220] K. Tahlan, R. Wilson, D.B. Kastrinsky, K. Arora, V. Nair, E. Fischer, S.W. Barnes, J.R. Walker, D. Alland, C.E. Barry, H.I. Boshoff, SQ109 Targets MmpL3, a Membrane Transporter of Trehalose Monomycolate Involved in Mycolic Acid Donation to the Cell Wall Core of Mycobacterium tuberculosis, Antimicrobial Agents and Chemotherapy, 56 (2012) 1797-1809.

[221] E.J. North, M.S. Scherman, D.F. Bruhn, J.S. Scarborough, M.M. Maddox, V. Jones, A. Grzegorzewicz, L. Yang, T. Hess, C. Morisseau, M. Jackson, M.R. McNeil, R.E. Lee, Design, synthesis and anti-tuberculosis activity of 1adamantyl-3-heteroaryl ureas with improved in vitro pharmacokinetic properties, Bioorganic \& Medicinal Chemistry, 21 (2013) 2587-2599.

[222] D.A. Smith, K. Beaumont, T.S. Maurer, L. Di, Volume of Distribution in Drug Design, Journal of Medicinal Chemistry, 58 (2015) 5691-5698.

[223] C. Lipinski, Drug-like properties and the causes of poor solubility and poor permeability, J. Pharmacol. Tox. Meth., 44 (2000) 235-249.

[224] J.J. Salomon, P. Galeron, N. Schulte, P.R. Morow, D. Severynse-Stevens, H. Huwer, N. Daum, C.-M. Lehr, A.J. Hickey, C. Ehrhardt, Biopharmaceutical in vitro characterization of CPZEN-45, a drug candidate for inhalation therapy of tuberculosis, Therapeutic Delivery, 4 (2013) 915-923.

[225] A. Bermingham, J.P. Derrick, The folic acid biosynthesis pathway in bacteria: evaluation of potential for antibacterial drug discovery, BioEssays, 24 (2002) 637648.

[226] A.C. Palmer, R. Kishony, Opposing effects of target overexpression reveal drug mechanisms, Nat Commun, 5 (2014).

[227] A. Wilkin, J. Feinberg, Pneumocystis carinii pneumonia: a clinical review, American family physician, 60 (1999) 1699-1708, 1713-1694.

[228] W.T. Hughes, J. Killmar, Monodrug efficacies of sulfonamides in prophylaxis for Pneumocystis carinii pneumonia, Antimicrobial Agents and Chemotherapy, 40 (1996) 962-965.

[229] M.K. Yun, The identification, analysis and structure-based development of novel inhibitors of 6-hydroxymethyl-7,8-dihydropterin pyrophosphokinase., Bioorganic and Medicinal Chemistry, 22 (2014) 2157-2165.

[230] J. Blaszczyk, G. Shi, H. Yan, X. Ji, Catalytic Center Assembly of HPPK as Revealed by the Crystal Structure of a Ternary Complex at $1.25 \AA$ Resolution, Structure, 8 (2000) 1049-1058.

[231] J. Blaszczyk, Y. Li, Y. Wu, G. Shi, X. Ji, H. Yan, Essential Roles of a Dynamic Loop in the Catalysis of 6-Hydroxymethyl-7,8-dihydropterin Pyrophosphokinase, Biochemistry, 43 (2004) 1469-1477. 
[232] B. Xiao, G. Shi, J. Gao, J. Blaszczyk, Q. Liu, X. Ji, H. Yan, Unusual Conformational Changes in 6-Hydroxymethyl-7,8-dihydropterin

Pyrophosphokinase as Revealed by X-ray Crystallography and NMR, Journal of Biological Chemistry, 276 (2001) 40274-40281.

[233] G. Li, K. Felczak, G. Shi, H. Yan, Mechanism of the Conformational Transitions in 6-Hydroxymethyl-7,8-dihydropterin Pyrophosphokinase as Revealed by NMR Spectroscopy, Biochemistry, 45 (2006) 12573-12581.

[234] C.W.I.V. Pemble, P.K. Mehta, S. Mehra, Z. Li, A. Nourse, R.E. Lee, S.W. White, Crystal Structure of the 6-Hydroxymethyl-7,8-Dihydropterin

Pyrophosphokinase?Dihydropteroate Synthase Bifunctional Enzyme from Francisella tularensis, PLoS ONE, 5 (2010) e14165.

[235] M. Hennig, G.E. Dale, A. D’Arcy, F. Danel, S. Fischer, C.P. Gray, S. Jolidon, F. Müller, M.G.P. Page, P. Pattison, C. Oefner, The structure and function of the 6hydroxymethyl-7,8-dihydropterin pyrophosphokinase from Haemophilus influenzae1, Journal of Molecular Biology, 287 (1999) 211-219.

[236] D.K. Stammers, A. Achari, D.O.N. Somers, P.K. Bryant, J. Rosemond, D.L. Scott, J.N. Champness, $2.0 \AA \mathrm{X}$-ray structure of the ternary complex of 7,8dihydro-6-hydroxymethylpterinpyrophosphokinase from Escherichia coli with ATP and a substrate analogue, FEBS Letters, 456 (1999) 49-53.

[237] G. Shi, J. Blaszczyk, X. Ji, H. Yan, Bisubstrate Analogue Inhibitors of 6Hydroxymethyl-7,8-dihydropterin Pyrophosphokinase: Synthesis and Biochemical and Crystallographic Studies, Journal of Medicinal Chemistry, 44 (2001) 1364-1371.

[238] J. Blaszczyk, Y. Li, S. Cherry, J. Alexandratos, Y. Wu, G. Shaw, J.E. Tropea, D.S. Waugh, H. Yan, X. Ji, Structure and activity of Yersinia pestis 6hydroxymethyl-7,8-dihydropterin pyrophosphokinase as a novel target for the development of antiplague therapeutics, Acta Crystallographica Section D, 63 (2007) 1169-1177.

[239] B. Xiao, G. Shi, X. Chen, H. Yan, X. Ji, Crystal structure of 6-hydroxymethyl7,8-dihydropterin pyrophosphokinase, a potential target for the development of novel antimicrobial agents, Structure, 7 (1999) 489-496.

[240] A. Garçon, C. Levy, J.P. Derrick, Crystal Structure of the Bifunctional Dihydroneopterin Aldolase/6-hydroxymethyl-7,8-dihydropterin Pyrophosphokinase from Streptococcus pneumoniae, Journal of Molecular Biology, 360 (2006) 644-653.

[241] M.C. Lawrence, P. Iliades, R.T. Fernley, J. Berglez, P.A. Pilling, I.G. Macreadie, The Three-dimensional Structure of the Bifunctional 6-Hydroxymethyl-7,8Dihydropterin Pyrophosphokinase/Dihydropteroate Synthase of Saccharomyces cerevisiae, Journal of Molecular Biology, 348 (2005) 655-670.

[242] A. Bermingham, J.R. Bottomley, W.U. Primrose, J.P. Derrick, Equilibrium and Kinetic Studies of Substrate Binding to 6-Hydroxymethyl-7,8-dihydropterin Pyrophosphokinase fromEscherichia coli, Journal of Biological Chemistry, 275 (2000) 17962-17967.

[243] G. Shi, Y. Gong, A. Savchenko, J.G. Zeikus, B. Xiao, X. Ji, H. Yan, Dissecting the nucleotide binding properties of Escherichia coli 6-hydroxymethyl-7,8dihydropterin pyrophosphokinase with fluorescent 3 '(2)'-o-anthraniloyladenosine 
5'-triphosphate1, Biochimica et Biophysica Acta (BBA) - Protein Structure and Molecular Enzymology, 1478 (2000) 289-299.

[244] Y. Li, Y. Gong, G. Shi, J. Blaszczyk, X. Ji, H. Yan, Chemical Transformation Is Not Rate-Limiting in the Reaction Catalyzed by Escherichia coli 6Hydroxymethyl-7,8-dihydropterin Pyrophosphokinase, Biochemistry, 41 (2002) 8777-8783.

[245] J. Blaszczyk, Y. Li, G. Shi, H. Yan, X. Ji, Dynamic Roles of Arginine Residues 82 and 92 of Escherichia coli 6-Hydroxymethyl-7,8-dihydropterin Pyrophosphokinase: Crystallographic Studies, Biochemistry, 42 (2003) 15731580.

[246] J. Blaszczyk, G. Shi, Y. Li, H. Yan, X. Ji, Reaction Trajectory of Pyrophosphoryl Transfer Catalyzed by 6-Hydroxymethyl-7,8-Dihydropterin Pyrophosphokinase, Structure, 12 (2004) 467-475.

[247] S. Chhabra, O. Dolezal, B.M. Collins, J. Newman, J.S. Simpson, I.G. Macreadie, R. Fernley, T.S. Peat, J.D. Swarbrick, Structure of S. aureus HPPK and the Discovery of a New Substrate Site Inhibitor, PLoS ONE, 7 (2012) e29444.

[248] G. Shi, G. Shaw, Y.-H. Liang, P. Subburaman, Y. Li, Y. Wu, H. Yan, X. Ji, Bisubstrate analogue inhibitors of 6-hydroxymethyl-7,8-dihydropterin pyrophosphokinase: New design with improved properties, Bioorganic \& Medicinal Chemistry, 20 (2012) 47-57.

[249] G. Shi, G. Shaw, Y. Li, Y. Wu, H. Yan, X. Ji, Bisubstrate analog inhibitors of 6hydroxymethyl-7,8-dihydropterin pyrophosphokinase: New lead exhibits a distinct binding mode, Bioorganic \& Medicinal Chemistry, 20 (2012) 4303-4309.

[250] S. Chhabra, N. Barlow, O. Dolezal, M.K. Hattarki, J. Newman, T.S. Peat, B. Graham, J.D. Swarbrick, Exploring the Chemical Space around 8Mercaptoguanine as a Route to New Inhibitors of the Folate Biosynthesis Enzyme HPPK, PLoS ONE, 8 (2013) e59535.

[251] M.L. Dennis, N.P. Pitcher, M.D. Lee, A.J. DeBono, Z.-C. Wang, J.R. Harjani, R. Rahmani, B. Cleary, T.S. Peat, J.B. Baell, J.D. Swarbrick, Structural Basis for the Selective Binding of Inhibitors to 6-Hydroxymethyl-7,8-dihydropterin Pyrophosphokinase from Staphylococcus aureus and Escherichia coli, Journal of Medicinal Chemistry, (2016).

[252] C. Abad-Zapatero, J.T. Metz, Ligand efficiency indices as guideposts for drug discovery, Drug Discovery Today, 10 (2005) 464-469.

[253] P.J. Hajduk, J. Greer, A decade of fragment-based drug design: strategic advances and lessons learned, Nature reviews. Drug discovery, 6 (2007) 211-219.

[254] M. Congreve, G. Chessari, D. Tisi, A.J. Woodhead, Recent Developments in Fragment-Based Drug Discovery, Journal of Medicinal Chemistry, 51 (2008) 3661-3680.

[255] P.A. Masters, T.A. O'Bryan, J. Zurlo, D.Q. Miller, N. Joshi, TRimethoprimsulfamethoxazole revisited, Archives of Internal Medicine, 163 (2003) 402-410.

[256] O.H. Cissé, M. Pagni, P.M. Hauser, Comparative Genomics Suggests That the Human Pathogenic Fungus Pneumocystis jirovecii Acquired Obligate Biotrophy through Gene Loss, Genome Biology and Evolution, 6 (2014) 1938-1948.

[257] M. Graffner-Nordberg, K. Kolmodin, J. Åqvist, S.F. Queener, A. Hallberg, Design, Synthesis, Computational Prediction, and Biological Evaluation of Ester 
Soft Drugs as Inhibitors of Dihydrofolate Reductase from Pneumocystis carinii, Journal of Medicinal Chemistry, 44 (2001) 2391-2402.

[258] V. Cody, J. Pace, J. Makin, J. Piraino, S.F. Queener, A. Rosowsky, Correlations of Inhibitor Kinetics for Pneumocystis jirovecii and Human Dihydrofolate Reductase with Structural Data for Human Active Site Mutant Enzyme Complexes, Biochemistry, 48 (2009) 1702-1711.

[259] D. Brown, Antibiotic resistance breakers: can repurposed drugs fill the antibiotic discovery void?, Nature reviews. Drug discovery, 14 (2015) 821-832.

[260] S. Nwaka, A. Hudson, Innovative lead discovery strategies for tropical diseases, Nature reviews. Drug discovery, 5 (2006) 941-955.

[261] E. Amata, H. Xi, G. Colmenarejo, R. Gonzalez-Diaz, C. Cordon-Obras, M. Berlanga, P. Manzano, J. Erath, N.E. Roncal, P.J. Lee, S.E. Leed, A. Rodriguez, R.J. Sciotti, M. Navarro, M.P. Pollastri, Identification of "Preferred" Human Kinase Inhibitors for Sleeping Sickness Lead Discovery. Are Some Kinases Better than Others for Inhibitor Repurposing?, ACS Infectious Diseases, 2 (2016) 180-186.

[262] Y. Zhao, D. Hammoudeh, W. Lin, S. Das, M.-K. Yun, Z. Li, E. Griffith, T. Chen, S.W. White, R.E. Lee, Development of a Pterin-based Fluorescent Probe for Screening Dihydropteroate Synthase, Bioconjugate Chemistry, 22 (2011) 21102117.

[263] C. Dalvit, P.E. Fagerness, D.T.A. Hadden, R.W. Sarver, B.J. Stockman, FluorineNMR Experiments for High-Throughput Screening: Theoretical Aspects, Practical Considerations, and Range of Applicability, Journal of the American Chemical Society, 125 (2003) 7696-7703.

[264] R. Jafari, H. Almqvist, H. Axelsson, M. Ignatushchenko, T. Lundbäck, P. Nordlund, D.M. Molina, The cellular thermal shift assay for evaluating drug target interactions in cells, Nat. Protocols, 9 (2014) 2100-2122.

[265] P.D. Butcher, Microarrays for Mycobacterium tuberculosis, Tuberculosis, 84 (2004) 131-137.

[266] R. Tommasi, D.G. Brown, G.K. Walkup, J.I. Manchester, A.A. Miller, ESKAPEing the labyrinth of antibacterial discovery, Nature reviews. Drug discovery, 14 (2015) 529-542.

[267] T.J. Ritchie, S.J.F. Macdonald, The impact of aromatic ring count on compound developability - are too many aromatic rings a liability in drug design?, Drug Discovery Today, 14 (2009) 1011-1020.

[268] F. Lovering, J. Bikker, C. Humblet, Escape from Flatland: Increasing Saturation as an Approach to Improving Clinical Success, Journal of Medicinal Chemistry, 52 (2009) 6752-6756.

[269] S.J. Projan, Why is big Pharma getting out of antibacterial drug discovery?, Current Opinion in Microbiology, 6 (2003) 427-430.

[270] S.T. Cole, R. Brosch, J. Parkhill, T. Garnier, C. Churcher, D. Harris, S.V. Gordon, K. Eiglmeier, S. Gas, C.E. Barry, F. Tekaia, K. Badcock, D. Basham, D. Brown, T. Chillingworth, R. Connor, R. Davies, K. Devlin, T. Feltwell, S. Gentles, N. Hamlin, S. Holroyd, T. Hornsby, K. Jagels, A. Krogh, J. McLean, S. Moule, L. Murphy, K. Oliver, J. Osborne, M.A. Quail, M.A. Rajandream, J. Rogers, S. Rutter, K. Seeger, J. Skelton, R. Squares, S. Squares, J.E. Sulston, K. Taylor, S. 
Whitehead, B.G. Barrell, Deciphering the biology of Mycobacterium tuberculosis from the complete genome sequence, Nature, 393 (1998) 537-544.

[271] E.D. Brown, G.D. Wright, New targets and screening approaches in antimicrobial drug discovery, Chemical reviews, 105 (2005) 759-774.

[272] T. Wunberg, M. Hendrix, A. Hillisch, M. Lobell, H. Meier, C. Schmeck, H. Wild, B. Hinzen, Improving the hit-to-lead process: data-driven assessment of drug-like and lead-like screening hits, Drug Discovery Today, 11 (2006) 175-180.

[273] M. Elinder, M. Geitmann, T. Gossas, P. Källblad, J. Winquist, H. Nordström, M. Hämäläinen, U.H. Danielson, Experimental Validation of a Fragment Library for Lead Discovery Using SPR Biosensor Technology, Journal of Biomolecular Screening, 16 (2011) 15-25.

[274] J.R. Brown, E.J. North, J.G. Hurdle, C. Morisseau, J.S. Scarborough, D. Sun, J. Kordulakova, M.S. Scherman, V. Jones, A. Grzegorzewicz, R.M. Crew, M. Jackson, M.R. McNeil, R.E. Lee, The structure-activity relationship of urea derivatives as anti-tuberculosis agents, Bioorg Med Chem, 19 (2011) 5585-5595.

[275] K.A. Abrahams, J.A.G. Cox, V.L. Spivey, N.J. Loman, M.J. Pallen, C. Constantinidou, R. Fernández, C. Alemparte, M.J. Remuiñán, D. Barros, L. Ballell, G.S. Besra, Identification of Novel Imidazo[1,2-a]pyridine Inhibitors Targeting M. tuberculosis QcrB, PLoS ONE, 7 (2012) e52951.

[276] G.M. Keseru, G.M. Makara, The influence of lead discovery strategies on the properties of drug candidates, Nature reviews. Drug discovery, 8 (2009) 203-212.

[277] C. Lipinski, Experimental and computational approaches to estimate solubility and permeability in drug discovery and development settings Advanced Drug Delivery Reviews, 23 (1997) 3-25.

[278] A. Maitra, S. Bates, T. Kolvekar, P.V. Devarajan, J.D. Guzman, S. Bhakta, Repurposing - a ray of hope in tackling extensively drug resistance in tuberculosis, International Journal of Infectious Diseases, 32 (2015) 50-55.

[279] D. Zhang, Y. Wang, J. Lu, Y. Pang, In vitro activity of $\beta$-lactams in combination with $\beta$-lactamase inhibitors against multidrug-resistant Mycobacterium tuberculosis isolates, Antimicrobial Agents and Chemotherapy, (2015).

[280] S. Hazra, H. Xu, J.S. Blanchard, Tebipenem, a New Carbapenem Antibiotic, Is a Slow Substrate That Inhibits the $\beta$-Lactamase from Mycobacterium tuberculosis, Biochemistry, 53 (2014) 3671-3678. 


\section{VITA}

Daniel Towner Hoagland was born in April 1988 in Louisville, Kentucky. Daniel graduated Wingate University in 2011 with a Bachelor of Science in Chemistry with a minor in Mathematics while being a member of the men's swimming team from 20072009. After graduation Daniel joined the University of Tennessee Health Science's Pharmaceutical Science department and Dr. Richard Lee's lab at St Jude Children's Research Hospital in the summer of 2011. During his time in the Lee lab Daniel placed first in the inaugural Chemical Biology and Therapeutics retreat scientific poster competition, was awarded multiple travel awards to attend scientific conferences across the world and published three papers to date. Daniel was selected as a Chair for the first Gordon Research Seminar associated with the Gordon Research Conference on New Antibacterial Discovery and Development, where he was directly involved in the meeting planning and helped make the seminar free for all accepted graduate students and postdocs to attend. Daniel has had many teaching responsibilities at the University of Tennessee Health Science Center School of Pharmacy, including a head teaching assistant role he held from 2014-2015. After the final approval of his dissertation, Daniel will be conferred a doctor of philosophy in pharmaceutical sciences and will graduate in August of 2016 from the University of Tennessee Health Science 